\title{
Modelos multiníveis Weibull com efeitos aleatórios
}

\author{
Freddy Hernández Barajas
}

TESE APRESENTADA

$\mathrm{AO}$

Instituto DE MATEMÁticA E ESTATÍSTICA

DA

UniversidAdE DE SÃo PAUlO

PARA

OBTENÇÃO DO TÍTULO

$\mathrm{DE}$

DOUTOR EM CIÊNCIAS

Programa: Estatística

Orientadora: Prof. Dra. Viviana Giampaoli

Durante o desenvolvimento deste trabalho o autor recebeu auxílio financeiro da $\mathrm{CAPES} / \mathrm{CNPq}$

São Paulo, 01 de maio de 2013 


\section{Modelos multiníveis Weibull com efeitos aletórios}

Esta versão da tese contém as correções e alterações sugeridas pela Comissão Julgadora durante a defesa da versão original do trabalho, realizada em 28/02/2013. Uma cópia da versão original está disponível no Instituto de Matemática e Estatística da Universidade de São Paulo.

Comissão Julgadora:

- Profa. Dra. Viviana Giampaoli (orientadora) - IME-USP

- Profa. Dra. Gisela Tunes da Silva - IME-USP

- Prof. Dr. Mário de Castro Andrade Filho - ICMC - USP

- Profa. Dra. Audrey Helen Mariz de Aquino Cysneiros - UFPE

- Profa. Dra. Michelli Karinne Barros da Silva - UFCG 


\section{Dedicatoria}

Dedico este trabalho aos meus pais, aos meus irmãos, e a minha esposa. 


\section{Agradecimentos}

Gostaria de agradecer aos meus familiares e especialmente a minha esposa quem foi meu apoio nesses quatro anos no Brasil.

Agradeço também à professora Viviana Giampaoli pelas suas orentações nesse trabalho.

Agradeço aos amigos e professores do Instituto de Matemática e Estatística IME USP.

Agradeço também aos membros da banca julgadora pelas sugestões. 


\section{Resumo}

Os modelos multiníveis são uma classe de modelos úteis na análise de bases de dados com estrutura hierárquica. No presente trabalho propõem-se os modelos multiníveis com resposta Weibull, nos quais são considerados interceptos aleatórios na modelagem dos dois parâmetros da distribuição da variável resposta. Os modelos aqui propostos são flexíveis devido a que a distribuição dos interceptos aleatórios pode der escolhida entre uma das seguintes quatro distribuições: normal, log-gama, logística e Cauchy. Uma extensão dos modelos é apresentada na qual é possível incluir na parte sistemática dos dois parâmetros da distribuição da variável resposta interceptos e inclinações aleatórias com distribuição normal bivariada. A estimação dos parâmetros é realizada pelo método de máxima verossimilhança usando a quadratura de Gauss-Hermite para aproximar a função de verossimilhança. Um pacote em linguagem $\mathrm{R}$ foi desenvolvido especialmente para a estimação dos parâmetros, predição dos efeitos aleatórios e para a obtenção dos resíduos nos modelos propostos. Adicionalmente, por meio de um estudo de simulação foi avaliado o impacto nas estimativas dos parâmetros do modelo ao assumir incorretamente a distribuição dos interceptos aleatórios.

Palavras-chave: modelos multiníveis, distribuição Weibull, quadratura de Gauss-Hermite. 


\section{Abstract}

Multilevel models are a class of models useful in the analysis of datasets with hierarchical structure. In the present work we propose multilevel Weibull models in which random intercepts are considered to model the two parameters of the Weibull distribution. The proposed models are flexible due to random intercepts distribution can be chosen from one of the four following distributions: normal, log-gamma, logistics and Cauchy. An extension of the models is presented in which we can include, in the systematic part of the two parameters of the distribution, random intercepts and slopes with a bivariate normal distribution. The parameter estimation is performed by maximum likelihood method using the Gauss Hermite quadrature to approximate the likelihood function. A package in $\mathrm{R}$ language was especially developed to obtain parameter estimation, random effects predictions and residuals for the proposed models. Additionally, through a simulation study we investigated the misspecification random effect distribution on estimated parameter for the proposed model.

Keywords: multilevel models, Weibull distribution, Gauss Hermite quadrature, survival analysis. 


\section{Sumário}

Lista de Figuras $\quad$ xiii

Lista de Tabelas $\quad$ xvii

1 Introdução $\quad 1$

2 Modelos multiníveis $\quad 3$

3 Modelo Multinível Weibull com dois níveis e interceptos aleatórios normais 9

3.1 Distribuição Weibull . . . . . . . . . . . . . . . . . 9 9

3.2 Apresentação do modelo . . . . . . . . . . . . . . . . . . 10

3.3 Estimação dos parâmetros do modelo . . . . . . . . . . . . . . . . 11

3.3.1 Função escore e matriz hessiana . . . . . . . . . . . . . . . . . 14

3.4 Predição dos efeitos aleatórios . . . . . . . . . . . . . . . 15

3.5 Proposta de Resíduos . . . . . . . . . . . . . . . . . 16

3.5.1 Resíduo Ordinário Padronizado . . . . . . . . . . . . . . 16

3.5.2 Resíduo Componente do Desvio . . . . . . . . . . . . . . . 16

3.5.3 Resíduo Quantil Normal Aleatorizado . . . . . . . . . . . . . . . 17

3.6 Teste sobre as componentes de variância . . . . . . . . . . . . . . 17

3.6.1 Teste da razão de verossimilhanças . . . . . . . . . . . . . . 18

3.7 Estudo de simulação . . . . . . . . . . . . . . . . . . . . . . 19

3.7.1 Processo de estimação . . . . . . . . . . . . . . . 20

3.7 .2 Distribuição empírica dos estimadores . . . . . . . . . . . . . . 20

3.7 .3 Distribuição empírica dos resíduos . . . . . . . . . . . . . . . . 21

3.7.4 Distribuição empírica da estatística da RV . . . . . . . . . . . . 23

3.8 Aplicações . . . . . . . . . . . . . . . . . . . . 25

3.8.1 Exemplo com base de dados simulada . . . . . . . . . . . . 26

3.8.2 Exemplo sobre transplante de medula óssea para pacientes com leucemia 28

3.9 Conclusões . . . . . . . . . . . . . . . . . . . . . . . . . 31

4 Modelo multinível Weibull com dois níveis e interceptos aleatórios loggama $\quad 41$

4.1 Apresentação do modelo . . . . . . . . . . . . . . . . . . 41 
4.2 Estimação dos parâmetros do modelo . . . . . . . . . . . . . . . . 42

4.3 Predição dos efeitos aleatórios e proposta de resíduos . . . . . . . . . . . 45

4.4 Aplicações . . . . . . . . . . . . . . . . . . . . . . . 45

4.4.1 Exemplo com base de dados simulada . . . . . . . . . . . 45

4.5 Estudo de simulação . . . . . . . . . . . . . . . . . . . . 47

4.5.1 Processo de estimação . . . . . . . . . . . . . . 48

4.5.2 Distribuição empírica de cada uma das componentes . . . . . . . . . . . 48

4.5 .3 Distribuição empírica dos resíduos . . . . . . . . . . . . . . . 49

4.6 Conclusões . . . . . . . . . . . . . . . . . . . . . . 52

5 Extensões do modelo multinível Weibull $\quad 59$

5.1 Flexibilização da distribuição dos interceptos aleatórios . . . . . . . . . . 59

5.2 Generalização do modelo multinível Weibull . . . . . . . . . . . . . . . 60

5.3 Conclusões . . . . . . . . . . . . . . . . . . . . . 62

6 Impacto de uma incorreta especificação na distribuição dos efeitos aleató$\begin{array}{ll}\text { rios } & 63\end{array}$

6.1 Estrutura do modelo . . . . . . . . . . . . . . . . 66

6.2 Estudo de simulação . . . . . . . . . . . . . . . . . 67

6.3 Conclusões . . . . . . . . . . . . . . . . . . . . 69

7 Aspectos computacionais $\quad 73$

7.1 Quadratura Gauss-Hermite . . . . . . . . . . . . . . . 73

7.2 Melhorando a QGHA com a eliminação de pontos . . . . . . . . . . 78

8 Pacote 'wlmm' $\quad 81$

$\begin{array}{lll}9 & \text { Conclusões } & 91\end{array}$

$\begin{array}{ll}\text { A Parametrizações da distribuição Weibull } & 93\end{array}$

$\begin{array}{ll}\text { B Bases de dados } & 97\end{array}$

C Função escore e matriz hessiana para o modelo Weibull com interceptos $\begin{array}{ll}\text { aleatórios normais } & 99\end{array}$

C.1 Função escore . . . . . . . . . . . . . . . . . . . . . . . . . 99

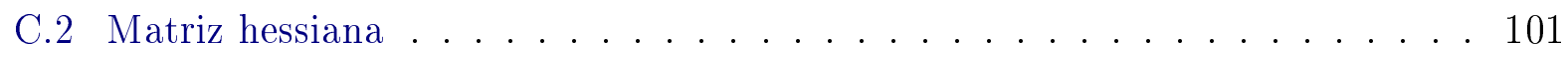

$\begin{array}{ll}\text { Referências Bibliográficas } & 107\end{array}$ 


\section{Lista de Figuras}

2.1 Diagrama de dispersão para $y$ dado $x \ldots \ldots \ldots \ldots \ldots$

2.2 Diagrama de dispersão para $y$ dado $x$ nas turmas 1 e $2 \ldots \ldots$

3.1 Estrutura hierárquica para o modelo com dois níveis. . . . . . . . . . . . 11

3.2 Função densidade estimada para as componentes de $\hat{\boldsymbol{\theta}}$ no modelo Weibull normal. A linha contínua corresponde ao caso de $N=10$ e $n_{i}=3$. A linha tracejada corresponde ao caso de $N=50$ e $n_{i}=10$. A linha tracejada vertical escura corresponde a cada um dos valores verdadeiros de $\boldsymbol{\theta}$. . . . . . . .

3.3 Gráfico normal de probabilidade para o resíduo $r_{o}$ no modelo Weibull normal com diferentes percentagens de censura para $N=5$ e $N=10$. . . . . . 22

3.4 Gráfico normal de probabilidade para o resíduo $r_{o}$ no modelo Weibull normal com diferentes percentagens de censura para $N=25$ e $N=50 \ldots$. . . .

3.5 Gráfico normal de probabilidade para o resíduo $r_{q}$ no modelo Weibull normal com diferentes percentagens de censura para $N=5$ e $N=10$. . . . . . . 24

3.6 Gráfico normal de probabilidade para o resíduo $r_{q}$ no modelo Weibull normal com diferentes percentagens de censura para $N=25$ e $N=50$. . . . . .

3.7 Gráfico normal de probabilidade para o resíduo $r_{d}$ no modelo Weibull normal com diferentes percentagens de censura para $N=5$ e $N=10$. . . . . .

3.8 Gráfico normal de probabilidade para o resíduo $r_{d}$ no modelo Weibull normal com diferentes percentagens de censura para $N=25$ e $N=50$. . . . . .

3.9 Gráfico normal de probabilidade para o resíduo $r_{M}$ no modelo Weibull normal com diferentes percentagens de censura para $N=5$ e $N=10$. . . . . .

3.10 Gráfico normal de probabilidade para o resíduo $r_{M}$ no modelo Weibull normal com diferentes percentagens de censura para $N=25$ e $N=50$. . . . .

3.11 Gráfico de probabilidade das estatísticas $\xi_{R V}$ versus os percentis da $0.5 \chi_{0}^{2}+$ $0.5 \chi_{1}^{2}$ para as combinações de $N=5$ e $n_{i}=3,5,10,15$ com $\tau_{1}=\tau_{2}=0$. . .

3.12 Gráfico de probabilidade das estatísticas $\xi_{R V}$ versus os percentis da $0.5 \chi_{0}^{2}+$ $0.5 \chi_{1}^{2}$ para as combinações de $N=10$ e $n_{i}=3,5,10,15$ com $\tau_{1}=\tau_{2}=0 . \quad$.

3.13 Gráfico de probabilidade das estatísticas $\xi_{R V}$ versus os percentis da $0.5 \chi_{0}^{2}+$ $0.5 \chi_{1}^{2}$ para as combinações de $N=25$ e $n_{i}=3,5,10,15$ com $\tau_{1}=\tau_{2}=0$. . 
3.14 Gráfico de probabilidade das estatísticas $\xi_{R V}$ versus os percentis da $0.5 \chi_{0}^{2}+$ $0.5 \chi_{1}^{2}$ para as combinações de $N=50$ e $n_{i}=3,5,10,15 \operatorname{com} \tau_{1}=\tau_{2}=0 . \quad$.

3.15 Estimativa da função densidade para a variável resposta $y$ da base de dados simulada com resposta Weibull e efeitos aleatórios normal. . . . . . . . . .

3.16 Gráficos normais de probabilidade dos efeitos aleatórios preditos $u_{1}$ e $u_{2}$ para a base de dados simulada com resposta Weibull e efeitos aleatórios normal. .

3.17 Gráficos normais de probabilidade dos resíduos $r_{o}, r_{d}$ e $r_{q}$ para base de dados simulada com resposta Weibull e efeitos aleatórios normal.

3.18 Gráfico de caixa para tempo até o transplante dados os hospitais.

3.19 Gráficos normais de probabilidade dos resíduos $r_{o}, r_{d}$ e $r_{q}$ para a base de dados leucemia. . . . . . . . . . . . . . . . . . .

4.1 Função densidade e gráfico de caixa para a variável resposta $y$ da base de dados simulada com resposta Weibull e efeitos aleatórios log-gama. . . . . .

4.2 Gráfico de probabilidade dos efeitos aleatórios preditos $\tilde{u}_{1}$ e $\tilde{u}_{2}$ para a base de dados simulada com resposta Weibull e efeitos aleatórios log-gama. . . . . .

4.3 Gráficos normais de probabilidade dos resíduos $r_{o}, r_{d}$ e $r_{q}$ para base de dados simulada com resposta Weibull e efeitos aleatórios log-gama. . . . . . . . .

4.4 Função densidade estimada para as componentes de $\hat{\boldsymbol{\theta}}$ no modelo Weibull com efeitos aleatórios log-gama. A linha contínua corresponde ao caso de $N=5$ e $n_{i}=5$. A linha tracejada corresponde ao caso de $N=25$ e $n_{i}=10$. A linha tracejada vertical escura corresponde a cada um dos valores verdadeiros de $\boldsymbol{\theta}$.

4.5 Gráfico normal de probabilidade para o resíduo $r_{o}$ no modelo Weibull loggama com diferentes percentagens de censura para $N=5$ e $N=10$. . . .

4.6 Gráfico normal de probabilidade para o resíduo $r_{o}$ no modelo Weibull loggama com diferentes percentagens de censura para $N=25$ e $N=50$. . . .

4.7 Gráfico normal de probabilidade para o resíduo $r_{q}$ no modelo Weibull loggama com diferentes percentagens de censura para $N=5$ e $N=10$. . . .

4.8 Gráfico normal de probabilidade para o resíduo $r_{q}$ no modelo Weibull loggama com diferentes percentagens de censura para $N=25$ e $N=50$. . . .

4.9 Gráfico normal de probabilidade para o resíduo $r_{d}$ no modelo Weibull loggama com diferentes percentagens de censura para $N=5$ e $N=10$. . . .

4.10 Gráfico normal de probabilidade para o resíduo $r_{d}$ no modelo Weibull loggama com diferentes percentagens de censura para $N=25$ e $N=50$. . . .

4.11 Gráfico normal de probabilidade para o resíduo $r_{M}$ no modelo Weibull loggama com diferentes percentagens de censura para $N=5$ e $N=10$. . . . .

4.12 Gráfico normal de probabilidade para o resíduo $r_{M}$ no modelo Weibull loggama com diferentes percentagens de censura para $N=25$ e $N=50$. . . .

5.1 Função de densidade dos efeitos aleatórios para diferentes valores do parâme-

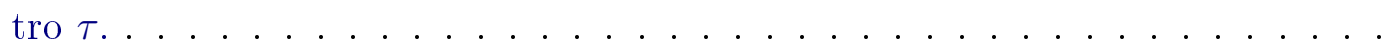


6.1 Distribuições consideradas para a geração dos efeitos aleatórios, cada uma com média 0 e variância unitária. . . . . . . . . . . . . 67

6.2 Mediana da distância relativa ente $\hat{\boldsymbol{\theta}}$ e $\boldsymbol{\theta}$ para cada uma das distribuições dos efeitos aleatórios com $\tau_{1}=\tau_{2}=1 \ldots \ldots \ldots$. . . . . . . 69

6.3 Mediana da distância relativa entre $\hat{\theta}_{k}$ e $\theta_{k}$ para cada uma das distribuições dos efeitos aleatórios com $\tau_{1}=\tau_{2}=1 \ldots \ldots \ldots$. . . . . . . 70

6.4 Mediana da distância relativa ente $\hat{\boldsymbol{\theta}}$ e $\boldsymbol{\theta}$ para cada uma das distribuições dos efeitos aleatórios com $\tau_{1}=\tau_{2}=1.5 \ldots \ldots \ldots$. . . . . . . . 71

6.5 Mediana da distância relativa entre $\hat{\theta}_{k}$ e $\theta_{k}$ para cada uma das distribuições dos efeitos aleatórios com $\tau_{1}=\tau_{2}=1.5 \ldots \ldots \ldots \ldots$. . . . . . . 72

7.1 Função $g_{1}(x)$ e pontos de quadratura. . . . . . . . . . . . . . . . . 74

7.2 Função $g_{2}(x)$ com os pontos de quadratura $p_{i}$ e $p_{i}^{\star} \ldots \ldots \ldots \ldots$

7.3 Curvas de nível para a função $g\left(x_{1}, x_{2}\right)$ com os pontos de quadratura $\boldsymbol{z}_{i}$ e $\boldsymbol{z}_{i}^{\star}$. $\quad 77$

7.4 Combinações dos pontos de quadratura sem e com a eliminação. . . . . . . 80

A.1 Funções de densidade, sobrevivência e risco da parameterização $W E I$ para diferentes valores de $\mu$ e $\sigma \ldots \ldots . \ldots . \ldots . \ldots . \ldots 94$

A.2 Funções de densidade, sobrevivência e risco da parameterização $W E I 2$ para diferentes valores de $\mu$ e $\sigma \ldots \ldots \ldots \ldots . \ldots \ldots 5$

A.3 Funções de densidade, sobrevivência e risco da parameterização $W E I 3$ para

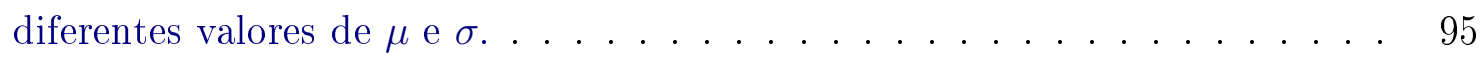




\section{Lista de Tabelas}

3.1 EQMM entre $\hat{\boldsymbol{\theta}}$ e $\boldsymbol{\theta}$ no estudo de simulação para o modelo Weibull normal. . 20

3.2 Média, desvio padrão, curtose e assimetria para $r_{o}$ no estudo de simulação do modelo Weibull normal com diferentes valores de $N, n_{i}$ e percentagem de

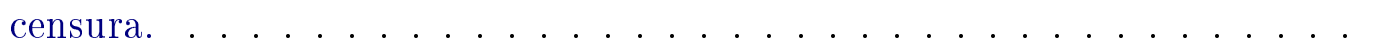

3.3 Média, desvio padrão, curtose e assimetria para $r_{q}$ no estudo de simulação do modelo Weibull normal com diferentes valores de $N, n_{i}$ e percentagem de

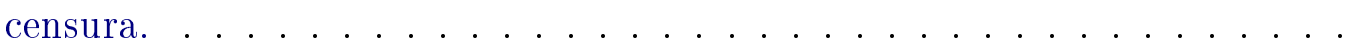

3.4 Média, desvio padrão, curtose e assimetria para $r_{d}$ no estudo de simulação do modelo Weibull normal com diferentes valores de $N, n_{i}$ e percentagem de censura. . . . . . . . . . . . . . . . . . .

3.5 Percetagem de rejeições de $H_{0}:\left(\tau_{1}, \tau_{2}\right)=0$ no teste RV a um nível de significância de $5 \%$. . . . . . . . . . . . . . . 37

3.6 Estimativas (e erros padrão em parênteses) para os parâmetros do modelo da base de dados simulada. . . . . . . . . . . . . . . . . . . . .

3.7 Estimativas e erros padrão dos parâmetros referentes ao modelo para a base de dados leucemia. . . . . . . . . . . . . . . . . . .

4.1 Estimativas e erros padrão (em parênteses) dos parâmetros usando a quadratura Gauss-Hermite adaptativa e não adaptativa para o modelo da base de dados simulada com resposta Weibull e efeitos aleatórios log-gama. . . . . . 46

4.2 EQMM entre $\hat{\boldsymbol{\theta}}$ e $\boldsymbol{\theta}$ no estudo de simulação para o modelo Weibull log-gama. 48

4.3 Média, desvio padrão, curtose e assimetria para $r_{o}$ no estudo de simulação do modelo Weibull log-gama com diferentes valores de $N, n_{i}$ e percentagem de

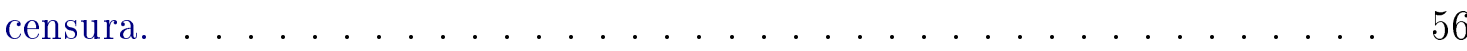

4.4 Média, desvio padrão, curtose e assimetria para $r_{q}$ no estudo de simulação do modelo Weibull log-gama com diferentes valores de $N, n_{i}$ e percentagem de censura. . . . . . . . . . . . . . . . . .

4.5 Média, desvio padrão, curtose e assimetria para $r_{d}$ no estudo de simulação do modelo Weibull log-gama com diferentes valores de $N, n_{i}$ e percentagem de censura. . . . . . . . . . . . . . . . . . . 
7.1 Número de combinações de pontos finais para solucionar $q=2,3$ integrais com $n=3,7,15$ pontos de quadratura. . . . . . . . . 80

B.1 Base de dados simulada multiníveis com dois níveis, resposta Weibull e efeitos aleatórios normais.

B.2 Base de dados simulada multinível com dois níveis, resposta Weibull e efeitos aleatórios log-gama. . . . . . . . . . . . . . . . 98 


\section{Capítulo 1}

\section{Introdução}

Neste trabalho propõem-se modelos multiníveis de dois níveis com resposta Weibull, nos quais os dois parâmetros da distribuição são modelados usando estruturas lineares. A ideia básica do trabalho é a junção do enfoque dos modelos multiníveis considerada por Raudenbush e Bryk (2002) e Goldstein (2011) com a abordagem dos modelos lineares aditivos generalizados para locação, escala e forma de Rigby e Stasinopoulos (2005), permitindo a possibilidade de incluir observações censuradas.

Dois casos principais são estudados para os modelos multiníveis Weibull propostos. O primeiro deles corresponde aos modelos lineares com interceptos aleatórios com distribuições normal, log-normal, logística ou Cauchy para os modelos dos dois parâmetros da distribuição Weibull. O segundo corresponde ao caso no qual tanto interceptos quanto inclinações aleatórias com distribuição normal bivariada podem ser consideradas na parte sistemática dos parâmetros da distribuição Weibull. A inferência nos modelos propostos é realizada sob uma abordagem marginal usando o método de máxima verossimilhança. Estudos de simulação Monte Carlo foram realizados com o intuito de avaliar as propostas apresentadas e de analisar o impacto nas estimativas do modelo multinível Weibull ao assumir incorretamente a distribuição dos interceptos aleatórios. Foi desenvolvido o pacote wlmmML na linguagem (R Development Core Team, 2012) para a análise dos modelos multiníveis Weibull.

O trabalho se inicia com uma revisão dos conceitos básicos dos modelos multiníveis no Capítulo 2. No Capítulo 3 é apresentado o modelo multinível Weibull com interceptos aleatórios seguindo distribuição normal. No Capítulo 4 é proposto o modelo multinível Weibull no qual os efeitos aleatórios têm distribuição log-gama. Duas extensões ao modelo multinível Weibull são apresentadas no Capítulo 5. A primeira considera interceptos aleatórios com distribuição não normal, especificamente distribuições logística e Cauchy. A segunda extensão corresponde ao modelo que inclue interceptos e inclinações aleatórias com distribuição normal bivariada na parte sistemática associada aos dois parâmetros da distribuição. Os resultados de um estudo de simulação para avaliar o impacto de assumir incorretamente a 
distribuição dos efeitos aleatórios são apresentados no Capítulo 6. Em seguida, no Capítulo 7 são expostos os conceitos principais sobre a quadratura de Gauss-Hermite, assim como uma nova proposta para diminuir o tempo requerido para sua aplicação. No Capítulo 8 é apresentado o pacote wlmmML desenvolvido para realizar este trabalho e no Capítulo 9 são apresentadas as conclusões relevantes do presente trabalho assim como os possíveis trabalhos futuros. 


\section{Capítulo 2}

\section{Modelos multiníveis}

Em estudos de educação e em diversas áreas da psicologia, os pesquisadores procuraram técnicas estatísticas que pudessem incorporar tanto informações sobre os indivíduos como informações sobre os grupos aos quais esses indivíduos pertencem. Em particular, existem pesquisas educacionais que têm bases de dados nas quais os alunos estão agrupados em turmas, e turmas agrupadas nas escolas, e talvez, as escolas agrupadas em distritos. Assim, é possível ter variáveis relacionadas a todos esses níveis, e o desafio é combinar todos os preditores em uma análise estatística apropriada, mais especificamente, uma análise de regressão e os modelos multiníveis são uma resposta para esse tipo de desafios (de Leeuw e Meijer, 2008).

Alguns dos primeiros trabalhos em modelos multiníveis na área educacional datam dos anos 80 do século XX e entre eles podem-se citar: Burstein (1980), Aitkin et al. (1981) e Aitkin e Longford (1986). Até meados de 1980, os problemas dos modelos multiníveis já estavam sendo estudados em outras áreas e sob outros nomes (de Leeuw e Meijer, 2008), alguns desses nomes e os trabalhos iniciais são modelos de efeitos mistos por Hartley e Rao (1967) e Laird e Ware (1982), regressão com coeficientes aleatórios por Rosenberg (1967), modelo de componentes de variância por Dempster et al. (1981) e Longford (1987), modelos lineares hierárquicos por Lindley e Smith (1972) e Smith (1973).

A modelagem multinível é uma área de pesquisa muito ativa, e é possível encontrar avanços em temas relacionados como: modelos multiníveis para variáveis ordinais e nominais em Hedeker (2008), modelos lineares generalizados multinível em Rodriguez (2008) e Skrondal e Rabe-Hesketh (2009), modelos multiníveis não hierárquicos em Rasbash e Browne (2008), modelos multiníveis com grupos pequenos em Raudenbush (2008), modelos elípticos multiníveis em Manghi (2011), entre outros. Em seguida, apresenta-se uma revisão do modelo multinível usual com dois níveis para resposta com distribuição normal com o objetivo de facilitar a leitura dos capítulos seguintes. 


\section{Modelos com dois níveis}

Na sequência, apresenta-se a estrutura dos modelos multiníveis como descrita em Raudenbush e Bryk (2002). O modelo mais simples e conhecido em regressão corresponde ao Modelo Linear Simples (MLS), que é representado pela seguinte expressão:

$$
y_{i}=\beta_{0}+\beta_{1} x_{i}+e_{i}
$$

em que $y_{i}$ e $x_{i}$ representam a variável resposta e a variável explicativa, respectivamente, para a observação $i$ com $i=1,2, \ldots, n$. Usualmente os erros $e_{i}$ são considerados independentes com $e_{i} \sim N\left(0, \sigma^{2}\right)$. Os estimadores para $\beta_{0}$ e $\beta_{1}$ podem ser obtidos usando, por exemplo, o Método de Máxima Verossimilhança (MMV) o pelo Método de Mínimos Quadrados (MMQ) e são denotados por $\hat{\beta}_{0}$ e $\hat{\beta}_{1}$, respetivamente.

Como exemplo, suponha-se que $x$ representa as notas obtidas por um conjunto de alunos provindos de diferentes turmas na prova de Matemática e $y$ representa as notas obtidas pelo mesmo grupo numa prova de Conhecimentos Gerais feita um ano depois da primeira prova. Assim, na Figura 2.1 apresenta-se um diagrama de dispersão para representar a situação hipotética descrita. A linha da figura representa a linha de tendência obtida pelo MMV.

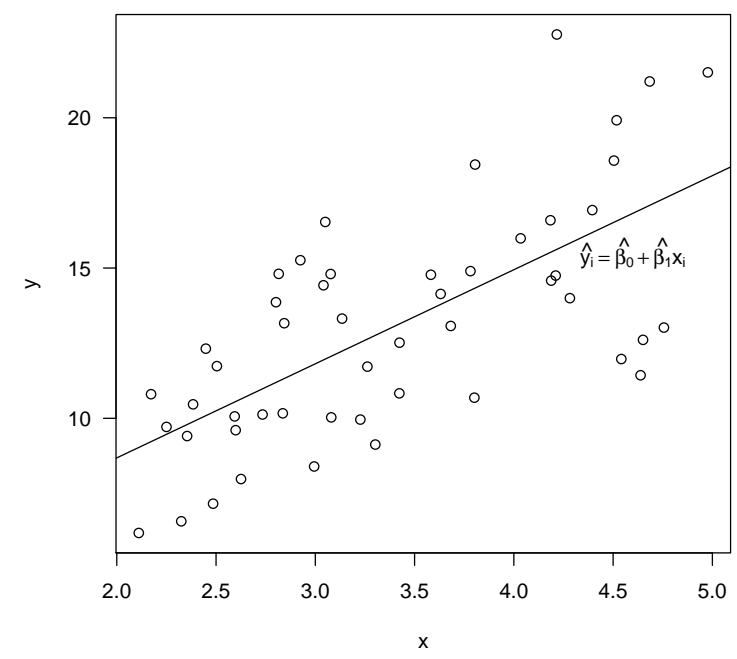

Figura 2.1: Diagrama de dispersão para $y$ dado $x$.

Suponha-se também que só duas turmas estão representadas na figura anterior. Assim, se representarmos as observações de cada turma de maneira diferente com suas próprias linhas de tendência, poderíamos obter uma situação como a apresentada na Figura 2.2. Observa-se que as linhas de tendência diferem para cada turma, assim, parece razoável considerar que os parâmetros $\beta_{0}$ e $\beta_{1}$ não são iguais para cada uma das turmas. 
Uma situação como da Figura 2.1 representa a mistura completa dos dados na qual são desconsiderados os agrupamentos, e uma análise nessa situação ignora a variação que existe na variável y nas duas turmas (Gelman e Hill, 2007). Por outro lado, uma situação como da Figura 2.2, que representa ajustes separados para cada um dos grupos, traz dificuldades na estimação quando existir um grande número de grupos ou quando existir um baixo número de observações por grupo (Raudenbush e Bryk, 2002). Os modelos multiníveis são considerados como uma mediação entre duas situações, a de mistura completa e a de ajustes individuais (Gelman e Hill, 2007).

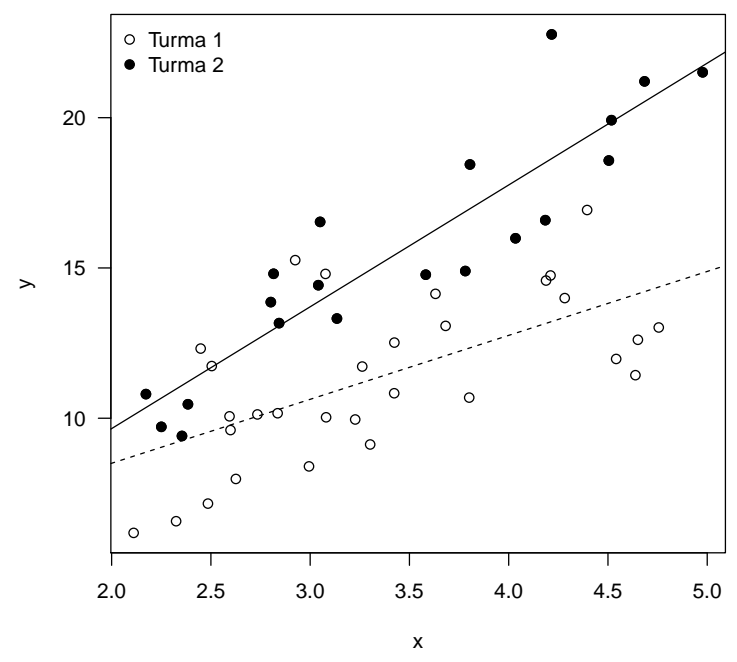

Figura 2.2: Diagrama de dispersão para y dado $x$ nas turmas 1 e 2

Os modelos multiníveis surgem como uma ferramenta para tratar os coeficientes que não são fixos numa regressão dadas variáveis explicativas medidas em nível dos indivíduos (Nível 1) ou variáveis explicativas medidas em nível dos grupos (Nível 2), criando com isto dois níveis de análise (de Leeuw e Meijer, 2008). Para exemplificar a forma de abordar o problema da diferença nos parâmetros entre os grupos apresenta-se um modelo com dois níveis, com uma única variável explicativa em cada nível. Sejam assim os seguintes modelos.

Modelo de nivel 1.

$$
y_{i j}=\beta_{0 i}+\beta_{1 i} x_{i j}+e_{i j}
$$

em que $i=1,2, \ldots, N$ representa os grupos, $j=1,2, \ldots, n_{i}$, sendo $n_{i}$ o número de observações em cada grupo e $x_{i j}$ corresponde à variável explicativa para a $j$-ésima observação do grupo $i$ medida no nível das observações (Nível 1). Novamente os termos de erro $e_{i j}$ são considerados como independentes e com distribuição normal. Assim, $e_{i j} \sim N\left(0, \sigma^{2}\right)$. No modelo (2.2) os parâmetros $\beta_{0 i}$ e $\beta_{1 i}$ dependem do índice $i$, significando que cada grupo terá seus próprios parâmetros. Uma suposição é que cada um destes parâmetros têm sua própria estrutura linear que pode ser função de uma variável explicativa medida em nível dos grupos. 
As estruturas lineares para $\beta_{0 i}$ e $\beta_{1 i}$ são as seguintes:

Modelo de nível 2.

$$
\begin{aligned}
& \beta_{0 i}=\gamma_{00}+\gamma_{01} w_{i}+u_{0 i}, \\
& \beta_{1 i}=\gamma_{10}+\gamma_{11} w_{i}+u_{1 i},
\end{aligned}
$$

em que $w_{i}$ corresponde a uma variável explicativa observada em nível dos grupos (Nível 2), com $u_{0 i}$ e $u_{1 i}$ independentes de $e_{i j}$. Os coeficientes $\gamma=\left(\gamma_{00}, \gamma_{01}, \gamma_{10}, \gamma_{11}\right)^{T}$ de $(2.3$ e 2.4) podem ser interpretados como segue:

- $\gamma_{00}$ : média de $\beta_{0 i}$ quando $w_{i}$ é zero;

- $\gamma_{01}$ : acréscimo na média para $\beta_{0 i}$ por cada unidade adicional em $w_{i}$;

- $\gamma_{10}$ : média para $\beta_{1 i}$ quando $w_{i}$ é zero;

- $\gamma_{11}$ : acréscimo na média para $\beta_{1 i}$ por cada unidade adicional em $w_{i}$;

- $u_{0 i}$ : efeito aleatório do $i$-ésimo grupo no intercepto $\beta_{0 i}$;

- $u_{1 i}$ : efeito aleatório do $i$-ésimo grupo na inclinação $\beta_{1 i}$.

Os elementos $u_{0 i}$ e $u_{1 i}$ correspondem aos termos de erro, os quais têm distribuição normal bivariada dada por

$$
\left(\begin{array}{l}
u_{0 i} \\
u_{1 i}
\end{array}\right) \sim N_{2}\left(\left(\begin{array}{l}
0 \\
0
\end{array}\right) ;\left(\begin{array}{cc}
\sigma_{u 0}^{2} & \sigma_{u 01} \\
\sigma_{u 10} & \sigma_{u 1}^{2}
\end{array}\right)\right)
$$

Ao substituir as equações (2.3 e 2.4) na equação (2.2) obtem-se o seguinte modelo combinado:

$$
y_{i j}=\gamma_{00}+\gamma_{10} x_{i j}+\gamma_{01} w_{i}+\gamma_{11} w_{i} x_{i j}+u_{0 i}+u_{1 i} x_{i j}+e_{i j} .
$$

No modelo combinado (2.6) tem-se que os termos $u_{0 i}$ e $u_{1 i}$ são dependentes para os elementos de um mesmo grupo. Portanto, não é possível proceder como no caso do modelo (2.1) para a estimação dos parâmetros $\left(\gamma_{00}, \gamma_{01}, \gamma_{10}, \gamma_{11}, \sigma_{u 0}^{2}, \sigma_{u 1}^{2}, \sigma_{u 01}\right)$ do modelo (Raudenbush e Bryk, 2002).

Um caso particular do modelo (2.2), que é conhecido como ANOVA com um fator, é caracterizado por não ter a variável explicativa $x_{i j}$ e porque o intercepto é dado por $\beta_{0 i}=$ $\gamma_{00}+u_{0 i}$. Outro caso particular do modelo (2.2) é quando existem $k$ variáveis explicativas no Nível 1 e sem nenhuma variável no Nível 2, assim, cada um dos coeficientes é dado por $\beta_{k i}=\gamma_{k 0}+u_{k i}$ e o modelo obtido é chamado de modelo com coeficientes aleatórios. Além das $k$ variáveis explicativas medidas em nível das observações (Nível 1), é possível incluir no 
modelo, para cada um dos coeficientes, um subconjunto de $m$ variáveis explicativas medidas em nível dos grupos (Nível 2). Assim, o modelo geral pode ser escrito como

$$
\begin{aligned}
& y_{i j}=\beta_{0 j}+\beta_{1 j} x_{1 i j}+\beta_{2 j} x_{2 i j}+\ldots+\beta_{k j} x_{k i j}+e_{i j}, \\
& \beta_{r j}=\gamma_{r 0}+\gamma_{r 1} w_{1 j}+\gamma_{r 2} w_{2 j}+\ldots+\gamma_{r m} w_{m j}+u_{r j} \quad \text { com } \quad r=0,1, \ldots, k
\end{aligned}
$$

O modelo (2.6) pode ser escrito na forma matricial como

$$
\boldsymbol{y}_{i}=\boldsymbol{X}_{i} \boldsymbol{\beta}+\boldsymbol{Z}_{i} \boldsymbol{u}_{i}+\boldsymbol{e}_{i}
$$

em que $\boldsymbol{y}_{i}$ corresponde ao vetor de observações para o grupo $i, \boldsymbol{X}_{i}$ e $\boldsymbol{Z}_{i}$ são matrizes de planejamento conhecidas do grupo $i$. Para o exemplo hipotético, relacionado às notas dos alunos, as ordens de $\boldsymbol{X}_{i}$ e $\boldsymbol{Z}_{i}$ são de $n_{i} \times 4$ e $n_{i} \times 2$ respectivamente, mas que podem ter outras ordens dependendo das estruturas de nível 1 e 2 consideradas. Em geral, as ordens são $n_{i} \times p$ e $n_{i} \times q$ para $\boldsymbol{X}_{i}$ e $\boldsymbol{Z}_{i}$ respectivamente. $\boldsymbol{\beta}$ é um vetor $p$ dimensional desconhecido de efeitos fixos enquanto $\boldsymbol{u}_{i}$ é um vetor $q$ dimensional de efeitos aleatórios com distribuição $N(\mathbf{0}, \boldsymbol{\Sigma})$. O vector $\boldsymbol{e}_{i}$ corresponde aos erros com distribuição $N\left(\mathbf{0}, \boldsymbol{D}_{i}\right)$, em que $\boldsymbol{D}_{i}$ pode ter alguma estrutura especial para levar em conta a correlação dos erros; caso não haja correlação, $\boldsymbol{D}_{i}=\sigma^{2} \boldsymbol{I}_{n i}$.

O modelo pode ser escrito na forma dos modelos lineares mistos de Laird e Ware (1982), dada por

$$
\begin{aligned}
\boldsymbol{y}_{i} & =\boldsymbol{X}_{i} \boldsymbol{\beta}+\boldsymbol{Z}_{i} \boldsymbol{u}_{i}+\boldsymbol{e}_{i}, \\
\boldsymbol{u}_{i} & \sim N(\mathbf{0}, \boldsymbol{\Sigma}), \\
\mathrm{e} \quad \boldsymbol{e}_{i} & \sim N\left(\mathbf{0}, \boldsymbol{D}_{i}\right)
\end{aligned}
$$

com $\boldsymbol{u}_{1}, \boldsymbol{u}_{2}, \ldots, \boldsymbol{u}_{N}, \boldsymbol{e}_{1}, \boldsymbol{e}_{2}, \ldots, \boldsymbol{e}_{N}$ independentes.

A distribuição do vetor $\boldsymbol{y}_{i}$ dado $\boldsymbol{u}_{i}$ é normal multivariada dada por

$$
\boldsymbol{y}_{i} \mid \boldsymbol{u}_{i} \sim N\left(\boldsymbol{X}_{i} \boldsymbol{\beta}+\boldsymbol{Z}_{i} \boldsymbol{u}_{i}, \boldsymbol{D}_{i}\right)
$$

A inferência, em geral, estão baseadas na distribuição marginal do vetor $\boldsymbol{y}_{i}$ (Molenberghs e Verbeke, 2005). Sejam $f_{i}\left(\boldsymbol{y}_{i} \mid \boldsymbol{u}_{i}\right)$ e $f\left(\boldsymbol{u}_{i}\right)$ as funções de densidade de (2.13 e 2.11), respectivamente. Assim, a distribuição marginal do vetor $\boldsymbol{y}_{i}$ é dada por

$$
f_{i}\left(\boldsymbol{y}_{i}\right)=\int_{\Re^{q}} f_{i}\left(\boldsymbol{y}_{i} \mid \boldsymbol{u}_{i}\right) f\left(\boldsymbol{u}_{i}\right) d \boldsymbol{u}_{i} .
$$

Prova-se que a densidade marginal de $\boldsymbol{y}_{i}$ no caso apresentado é $\boldsymbol{y}_{i} \sim N\left(\boldsymbol{X}_{i} \boldsymbol{\beta}, \boldsymbol{Z}_{i} \boldsymbol{\Sigma} \boldsymbol{Z}_{i}^{T}+\boldsymbol{D}_{i}\right)$, vide Molenberghs e Verbeke (2005). 
Denotando por $\boldsymbol{V}_{i}(\boldsymbol{\alpha})$ a matriz de covariânças da distribuição marginal do vetor $\boldsymbol{y}_{i}$, temse que $\boldsymbol{V}_{i}(\boldsymbol{\alpha})=\boldsymbol{Z}_{i} \boldsymbol{\Sigma} \boldsymbol{Z}_{i}^{T}+\boldsymbol{D}_{i}$, em que $\boldsymbol{\alpha}$ corresponde ao vetor de parâmetros desconhecidos denominado de componentes de variância. Assim, o vetor geral de parâmetros desconhecido do modelo é dado por $\boldsymbol{\theta}=(\boldsymbol{\beta}, \boldsymbol{\alpha})^{T}$. O vetor $\hat{\boldsymbol{\theta}}$ de estimativas de $\boldsymbol{\theta}$ é obtido usualmente usando o método de máxima verossimilhança ou o método de máxima verossimilhança restrita; para mais detalhes vide Verbeke e Molenberghs (2000) e Demidenko (2004). 


\section{Capítulo 3}

\section{Modelo Multinível Weibull com dois níveis e interceptos aleatórios normais}

Neste capítulo descreve-se o modelo multinível de dois níveis com variável resposta Weibull incluindo interceptos aleatórios com distribuição normal nos modelos relacionados aos dois parâmetros da distribuição da variável resposta. Posteriormente, apresenta-se o método para a estimação dos parâmetros do modelo. Na Seção 3.1 propõe-se a forma para predizer os efeitos aleatórios. Em seguida, propõem-se três tipos de resíduos para o modelo estudado. Na Seção 3.6 apresenta-se o teste de componentes de variância para decidir se é apropriado considerar ou não efeitos aleatórios nos modelos dos parâmetros da distribuição. Em particular, o teste é realizado por meio do teste da razão de verossimilhanças. Na Seção 3.7 apresentam-se os resultados de um estudo de simulação Monte Carlo realizado objetivando investigar a distribuição empírica tanto dos estimatimadores dos parâmetros quanto dos resíduos propostos, assim como o comportamento do teste de razão de verossimilhanças. Logo na Seção 3.8 são apresentadas duas aplicações do método proposto são apresentadas, a primeira delas com uma base de dados simulada e a segunda com uma base de dados real referente ao tempo para transplante de medula óssea em pacientes com leucemia.

\subsection{Distribuição Weibull}

Na literatura há várias parametrizações da distribuição Weibull que podem ser encontradas, por exemplo, em Stasinopoulos e Rigby (2012) e que são denotadas respectivamente por $W E I, W E I 2$ e WEI3. Uma descrição das três parametrizações pode ser encontrada no Apêndice A. Neste trabalho foi usada a versão WEI3, mas os resultados podem ser estendidos para as outras duas parametrizações de maneira direta. A função de densidade de probabilidade, o valor esperado, a variância, a função distribuição acumulada e a função de sobrevivência para a versão $W E I 3$ são dadas, respectivamente, por: 


$$
\begin{aligned}
f_{Y}(y \mid \mu, \sigma) & =\frac{\sigma}{\kappa}\left(\frac{y}{\kappa}\right)^{\sigma-1} \exp \left[-\left(\frac{y}{\kappa}\right)^{\sigma}\right], \quad \mu>0, \quad \sigma>0, \\
E(Y) & =\mu \\
\operatorname{Var}(Y) & =\mu^{2}\left[\Gamma\left(\frac{2}{\sigma}+1\right) \Gamma^{-2}\left(\frac{1}{\sigma}+1\right)-1\right], \\
F(y) & =1-\exp \left[-\left(\frac{y}{\kappa}\right)^{\sigma}\right], \\
S(y) & =\exp \left[-\left(\frac{y}{\kappa}\right)^{\sigma}\right]
\end{aligned}
$$

em que $\kappa=\mu / \Gamma(1 / \sigma+1)$ e $\Gamma(\cdot)$ corresponde à função gama. Nota-se que nessa parametrização $\mu$ coincide com $E(Y)$.

\subsection{Apresentação do modelo}

A estrutura hierárquica de dois níveis considerada para o modelo com resposta Weibull é representada na Figura 3.1. Denotando $y_{i j}$ como a $j$-ésima medida do $i$-ésimo grupo, a estrutura matemática do modelo é a seguinte:

$$
\begin{aligned}
y_{i j} \mid u_{1 i}, u_{2 i} & \stackrel{i n d}{\sim} W \operatorname{EI}\left(\mu_{i j}, \sigma_{i j}\right), \\
u_{1 i} & \stackrel{i i d}{\sim} N\left(0, \tau_{1}^{2}\right) \\
\mathrm{e} \quad u_{2 i} & \stackrel{i i d}{\sim} N\left(0, \tau_{2}^{2}\right), \quad i=1,2, \ldots, N, \quad j=1,2, \ldots, n_{i},
\end{aligned}
$$

em que os parâmetros $\mu$ e $\sigma$ são modelados, respectivamente, por

$$
\begin{aligned}
\log \left(\mu_{i j}\right) & =\boldsymbol{X}_{1 i, j}^{\top} \cdot \boldsymbol{\beta}_{1}+u_{1 i} \\
\text { e } \quad \log \left(\sigma_{i j}\right) & =\boldsymbol{X}_{2 i, j}^{\top} \cdot \boldsymbol{\beta}_{2}+u_{2 i} .
\end{aligned}
$$

As matrizes $\boldsymbol{X}_{1 i}$ e $\boldsymbol{X}_{2 i}$ são matrizes de planejamento conhecidas para o grupo $i$ enquanto $\boldsymbol{X}_{1 i, j}$. e $\boldsymbol{X}_{2 i, j}$. correspondem à $j$-ésima linha de $\boldsymbol{X}_{1 i}$ e $\boldsymbol{X}_{2 i}$, respectivamente; $\boldsymbol{\beta}_{1}$ e $\boldsymbol{\beta}_{2}$ são vetores de parâmetros fixos desconhecidos. Assim o modelo é determinado pela expressão (3.4) e as componentes sistemáticas por (3.5) e (3.6).

Em forma vetorial, os parâmetros $\mu$ e $\sigma$ para cada grupo têm a seguinte estrutura:

$$
\begin{aligned}
& \log \left(\boldsymbol{\mu}_{i}\right)=\boldsymbol{X}_{1 i} \boldsymbol{\beta}_{1}+\mathbf{1}_{n_{i}} u_{1 i}, \\
& \log \left(\boldsymbol{\sigma}_{i}\right)=\boldsymbol{X}_{2 i} \boldsymbol{\beta}_{2}+\mathbf{1}_{n_{i}} u_{2 i} .
\end{aligned}
$$


em que

$$
\begin{aligned}
\boldsymbol{\mu}_{i} & =\left(\mu_{i 1}, \mu_{i 2}, \ldots, \mu_{i n_{i}}\right)^{\top}, \\
\log \left(\boldsymbol{\mu}_{i}\right) & =\left(\log \left(\mu_{i 1}\right), \log \left(\mu_{i 2}\right), \ldots, \log \left(\mu_{i n_{i}}\right)\right)^{\top}, \\
\boldsymbol{\sigma}_{i} & =\left(\sigma_{i 1}, \sigma_{i 2}, \ldots, \sigma_{i n_{i}}\right)^{\top} \\
\text { e } \quad \log \left(\boldsymbol{\sigma}_{i}\right) & =\left(\log \left(\sigma_{i 1}\right), \log \left(\sigma_{i 2}\right), \ldots, \log \left(\sigma_{i n_{i}}\right)\right)^{\top},
\end{aligned}
$$

$\operatorname{com} \mathbf{1}_{n_{i}}$ indicando um vetor coluna de $1^{\prime} s$ de dimensão $n_{i}$. Note que até este ponto o modelo tem a mesma estrutura dos modelos lineares aditivos generalizados para locação, escala e forma (GAMLSS) propostos por Rigby e Stasinopoulos (2005).
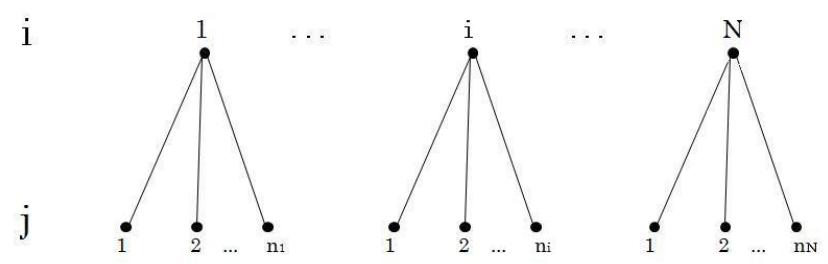

Figura 3.1: Estrutura hierárquica para o modelo com dois níveis.

\subsection{Estimação dos parâmetros do modelo}

As estimativas dos parâmetros do modelo são obtidas maximizando o logaritmo da função de verossimilhança, que é construída usando o enfoque marginal partindo da função densidade condicional do vetor de observações para para o $i$-ésimo grupo dados os efeitos aleatórios. Adicionalmente, no modelo é possível considerar observações censuradas à direita Tipo I utilizando uma variável indicadora $\delta$ definida como segue:

$$
\delta_{i j}= \begin{cases}0, & \text { se } y_{i j} \text { é censurada, } \\ 1, & \text { se } y_{i j} \text { não é censurada. }\end{cases}
$$

Assim, a função densidade condicional para o vetor de observações $\boldsymbol{y}_{i}=\left(y_{i 1}, \ldots, y_{i n_{i}}\right)^{\top}$ 
do $i$-ésimo grupo, dado $\boldsymbol{u}_{i}=\left(u_{1 i}, u_{2 i}\right)^{\top}$, tem expressão

$$
\begin{aligned}
P\left(\boldsymbol{y}_{i} \mid \boldsymbol{u}_{i}\right) & =\prod_{j=1}^{n_{i}}\left[f_{Y}\left(y_{i j} \mid \boldsymbol{u}_{i}\right)\right]^{\delta_{i j}}\left[S\left(y_{i j} \mid \boldsymbol{u}_{i}\right)\right]^{1-\delta_{i j}} \\
& =\prod_{j=1}^{n_{i}}\left[\frac{\sigma_{i j}}{\kappa_{i j}}\left(\frac{y_{i j}}{\kappa_{i j}}\right)^{\sigma_{i j}-1} \exp \left(-\left(\frac{y_{i j}}{\kappa_{i j}}\right)^{\sigma_{i j}}\right)\right]^{\delta_{i j}}\left[\exp \left(-\left(\frac{y_{i j}}{\kappa_{i j}}\right)^{\sigma_{i j}}\right)\right]^{1-\delta_{i j}} \\
& =\prod_{j=1}^{n_{i}}\left[\frac{\sigma_{i j}}{\kappa_{i j}}\left(\frac{y_{i j}}{\kappa_{i j}}\right)^{\sigma_{i j}-1}\right]^{\delta_{i j}} \exp \left(-\left(\frac{y_{i j}}{\kappa_{i j}}\right)^{\sigma_{i j}}\right) \\
& =\exp \sum_{j=1}^{n_{i}}\left[\delta_{i j} \log \left(\sigma_{i j} \kappa_{i j}^{-\sigma_{i j}} y_{i j}^{\sigma_{i j}-1}\right)-\kappa_{i j}^{-\sigma_{i j}} y_{i j}^{\sigma_{i j}}\right] \\
& =\exp \sum_{j=1}^{n_{i}}\left[\delta_{i j} \log \left(\sigma_{i j} \alpha_{i j} y_{i j}^{\sigma_{i j}-1}\right)-\alpha_{i j} y_{i j}^{\sigma_{i j}}\right] \\
& =\exp \left[\boldsymbol{\delta}_{i}^{\top}\left(\log \left(\boldsymbol{\sigma}_{i}\right)+\log \left(\boldsymbol{\alpha}_{i}\right)+\operatorname{diag}\left(\boldsymbol{\sigma}_{i}-\mathbf{1}_{n_{i}}\right) \log \left(\boldsymbol{y}_{i}\right)\right)-\boldsymbol{\alpha}_{i}^{\top} \boldsymbol{y}_{i}^{\star}\right] \\
& =\exp \left[g\left(\boldsymbol{y}_{i}, \boldsymbol{\delta}_{i}, \boldsymbol{u}_{i}\right)\right],
\end{aligned}
$$

em que

$$
\begin{aligned}
\alpha_{i j} & =\kappa_{i j}^{-\sigma_{i j}}, \\
\boldsymbol{\alpha}_{i} & =\left(\alpha_{i 1}, \alpha_{i 2}, \ldots, \alpha_{i n_{i}}\right)^{\top}, \\
\boldsymbol{\delta}_{i} & =\left(\delta_{i 1}, \delta_{i 2}, \ldots, \delta_{i n_{i}}\right)^{\top}, \\
\log \left(\boldsymbol{y}_{i}\right) & =\left(\log \left(y_{i 1}\right), \log \left(y_{i 2}\right), \ldots, \log \left(y_{i n_{i}}\right)\right)^{\top} \\
\mathrm{e} \quad \boldsymbol{y}_{i}^{\star} & =\left(y_{i 1}^{\sigma_{i 1}}, y_{i 2}^{\sigma_{i 2}}, \ldots, y_{i n_{i}}^{\sigma_{i n_{i}}}\right)^{\top},
\end{aligned}
$$

$\operatorname{com} g(\cdot)$ definida como

$$
g\left(\boldsymbol{y}_{i}, \boldsymbol{\delta}_{i}, \boldsymbol{u}_{i}\right)=\boldsymbol{\delta}_{i}^{\top}\left(\log \left(\boldsymbol{\sigma}_{i}\right)+\log \left(\boldsymbol{\alpha}_{i}\right)+\operatorname{diag}\left\{\boldsymbol{\sigma}_{i}-\mathbf{1}_{n_{i}}\right\} \log \left(\boldsymbol{y}_{i}\right)\right)-\boldsymbol{\alpha}_{i}^{\top} \boldsymbol{y}_{i}^{\star} .
$$

A função densidade conjunta do vetor de efeitos aleatórios é denotada por $P\left(\boldsymbol{u}_{i}\right)$ é corresponde ao produto de duas densidades normais de média nula. Assim, a função densidade conjunta do vetor $\left(\boldsymbol{y}_{i}, \boldsymbol{u}_{i}\right)$ é dada por

$$
\begin{aligned}
P\left(\boldsymbol{y}_{i}, \boldsymbol{u}_{i}\right) & =P\left(\boldsymbol{y}_{i} \mid \boldsymbol{u}_{i}\right) P\left(\boldsymbol{u}_{i}\right) \\
& =\exp \left[g\left(\boldsymbol{y}_{i}, \boldsymbol{\delta}_{i}, \boldsymbol{u}_{i}\right)\right]\left(2 \pi \tau_{1}^{2}\right)^{-1 / 2} \exp \left(-\frac{u_{1 i}^{2}}{2 \tau_{1}^{2}}\right)\left(2 \pi \tau_{2}^{2}\right)^{-1 / 2} \exp \left(-\frac{u_{2 i}^{2}}{2 \tau_{2}^{2}}\right) \\
& =\exp \left[g\left(\boldsymbol{y}_{i}, \boldsymbol{\delta}_{i}, \boldsymbol{u}_{i}\right)\right] \exp \left(-\frac{u_{1 i}^{2}}{2 \tau_{1}^{2}}-\frac{u_{2 i}^{2}}{2 \tau_{2}^{2}}\right) \frac{1}{2 \pi \tau_{1} \tau_{2}} .
\end{aligned}
$$

A função densidade marginal para o vetor $\boldsymbol{y}_{i}$ é obtida integrando (3.10) sobre os efeitos 
aleatórios $\boldsymbol{u}_{i}$ e é dada pela expressão

$$
\begin{aligned}
P\left(\boldsymbol{y}_{i}\right) & =\iint_{\Re^{2}} P\left(\boldsymbol{y}_{i}, \boldsymbol{u}_{i}\right) d u_{1 i} d u_{2 i} \\
& =\iint_{\Re^{2}} \exp \left[g\left(\boldsymbol{y}_{i}, \boldsymbol{\delta}_{i}, \boldsymbol{u}_{i}\right)\right] \exp \left(-\frac{u_{1 i}^{2}}{2 \tau_{1}^{2}}-\frac{u_{2 i}^{2}}{2 \tau_{2}^{2}}\right) \frac{d u_{1 i} d u_{2 i}}{2 \pi \tau_{1} \tau_{2}} .
\end{aligned}
$$

Como em muitas situações de modelos mistos, esta integral não tem uma expressão em forma fechada e aproximações são necessárias para obter uma estimativa computacionalmente viável (Pinheiro e Chao, 2006). Neste trabalho foi usado o método de aproximação denominado quadratura de Gauss-Hermite (QGH) que permite aproximar uma integral de uma função $g(x)$ com valores na reta por meio de uma soma ponderada, na qual a variável de interesse $x$ é substituída por $N_{Q G H}$, pontos de quadratura denotados por $p_{k}$ correspondentes às $N_{Q G H}$ raízes do polinômio de Hermite com pesos associados $w_{k}$. A aproximação da integral pelo método QGH é definida pela seguinte expressão:

$$
\int_{\Re} g(x) d x \approx \sum_{k=1}^{N_{Q G H}} g\left(p_{k}\right) \exp \left(p_{k}^{2}\right) w_{k}
$$

Uma explicação mais detalhada e com exemplos do método pode ser encontrada no Capítulo 7. Fazendo a mudança de variáveis $b_{1 i}=u_{1 i} /\left(\sqrt{2} \tau_{1}\right)$ e $b_{2 i}=u_{2 i} /\left(\sqrt{2} \tau_{2}\right)$ na expressão (3.11), obtém-se uma expressão apropriada para usar a aproximação QGH. Sendo $\boldsymbol{b}_{i}=\left(b_{1 i}, b_{2 i}\right)^{\top}$ o vetor das novas variáveis, a função densidade marginal obtida é a seguinte:

$$
P\left(\boldsymbol{y}_{i}\right)=\iint_{\Re^{2}} \exp \left[g\left(\boldsymbol{y}_{i}, \boldsymbol{\delta}_{i}, \sqrt{2} \boldsymbol{b}_{i}^{\top} \boldsymbol{T}\right)\right] \exp \left(-b_{1 i}^{2}-b_{2 i}^{2}\right) \frac{1}{\pi} d b_{1 i} d b_{2 i},
$$

em que $\boldsymbol{T}=\operatorname{diag}\left(\tau_{1}, \tau_{2}\right)$. Para aplicar a aproximação QGH a cada uma das integrais na expressão (3.12), a variável $b_{1 i}$ é substituída por $N_{Q G H}$ pontos de quadratura denotados por $p_{l}$ com pesos associados $w_{l}$, da mesma forma, a outra variável $b_{2 i}$ é substituída por $N_{Q G H}$ pontos de quadratura denotados por $p_{m}$ com pesos associados $w_{m}$. Sem perda de generalidade, aqui foi usado o mesmo número de pontos $N_{Q G H}$ de quadratura para $b_{1 i}$ e $b_{2 i}$. Uma maior precisão da aproximação da integral pode ser obtida aumentando o número de pontos de quadratura. No entanto, a aproximação de uma integral em $\Re^{k}$ precisaria de $N_{Q G H}^{k}$ pontos de grade o qual torna-se computacionalmente intensivo e com possíveis problemas se o aumento nos pontos de quadratura for exagerado. Na prática, $7<N_{Q G H}<13$ gera uma aproximação razoável para uma integral (Demidenko, 2004; Pinheiro e Chao, 2006). Dessa forma, a função densidade marginal aproximada para $\boldsymbol{y}_{i}$ usando QGH é dada por

$$
P\left(\boldsymbol{y}_{i}\right) \approx \sum_{l=1}^{N_{Q G H}} \sum_{m=1}^{N_{Q G H}} \exp \left[g\left(\boldsymbol{y}_{i}, \boldsymbol{\delta}_{i}, \sqrt{2} \boldsymbol{p}_{l, m}^{\top} \boldsymbol{T}\right)\right] \frac{w_{l} w_{m}}{\pi}
$$

em que $\boldsymbol{p}_{l, m}=\left(p_{l}, p_{m}\right)^{\top}$. 
Seja $\boldsymbol{\theta}=\left(\boldsymbol{\beta}_{1}^{\top}, \boldsymbol{\beta}_{2}^{\top}, \tau_{1}, \tau_{2}\right)^{\top}$ o vetor de parâmetros do modelo, que pertence ao espaço paramétrico $\Theta$, em que $\Theta=\Re^{p+q} \times \Re_{+}^{2}$, com $p$ e $q$ correspondendo ao número de efeitos fixos em $\mu$ e $\sigma$, respectivamente. Assim, o logaritmo da função de verossimilhança $l(\boldsymbol{\theta})$ para o modelo fica expressa como

$$
l(\boldsymbol{\theta})=\sum_{i=1}^{N} \log P\left(\boldsymbol{y}_{i}\right) .
$$

Para o logaritmo da função de verossimilhança (3.14), o vetor de primeiras derivadas e a matriz de segundas derivadas são úteis para o processo de estimação e obtenção dos erros padrão do vetor de parâmetros $\boldsymbol{\theta}$ e suas expressões são apresentadas a seguir.

\subsubsection{Função escore e matriz hessiana}

O vetor $\boldsymbol{U}(\boldsymbol{\theta})$ de primeiras derivadas da função de log-verossimilhança $l(\boldsymbol{\theta})$ com relação às componentes do vetor de parâmetros $\boldsymbol{\theta}$ têm as seguintes componentes:

$$
\begin{aligned}
\frac{\partial l(\boldsymbol{\theta})}{\partial \boldsymbol{\beta}_{1}} & =\sum_{i=1}^{N} \frac{\boldsymbol{X}_{1 i}^{\top} \boldsymbol{C}_{1 i}}{P_{i}}, \\
\frac{\partial l(\boldsymbol{\theta})}{\partial \boldsymbol{\beta}_{2}} & =\sum_{i=1}^{N} \frac{\boldsymbol{X}_{2 i}^{\top} \boldsymbol{C}_{2 i}}{P_{i}}, \\
\frac{\partial l(\boldsymbol{\theta})}{\partial \tau_{1}} & =\sum_{i=1}^{N} \frac{C_{3 i}}{P_{i}} \\
\mathrm{e} \quad \frac{\partial l(\boldsymbol{\theta})}{\partial \tau_{2}} & =\sum_{i=1}^{N} \frac{C_{4 i}}{P_{i}},
\end{aligned}
$$

em que $P_{i}=P\left(\boldsymbol{y}_{i}\right)$. As expressões para $\boldsymbol{C}_{1 i}, \boldsymbol{C}_{2 i}, C_{3 i}$ e $C_{4 i}$ são dadas no Apêndice C. Note que os elementos $\boldsymbol{C}_{1 i}$ e $\boldsymbol{C}_{2 i}$ são vetores enquanto $C_{3 i}$ e $C_{4 i}$ são escalares.

A matriz hessiana $H(\boldsymbol{\theta})$ da função de $\log$-verossimilhança $l(\boldsymbol{\theta})$ em relação às componentes 
do vetor de parâmetros $\boldsymbol{\theta}$ tem os seguintes elementos:

$$
\begin{aligned}
& \frac{\partial^{2} l(\boldsymbol{\theta})}{\partial \boldsymbol{\beta}_{1}^{2}}=\sum_{i=1}^{N} \boldsymbol{X}_{1 i}^{\top}\left[\frac{-\boldsymbol{C}_{1 i} \boldsymbol{C}_{1 i}^{\top}}{P_{i}^{2}}+\frac{\boldsymbol{H}_{1 i}}{P_{i}}\right] \boldsymbol{X}_{1 i}, \\
& \frac{\partial^{2} l(\boldsymbol{\theta})}{\partial \boldsymbol{\beta}_{2} \partial \boldsymbol{\beta}_{1}}=\sum_{i=1}^{N} \boldsymbol{X}_{1 i}^{\top}\left[\frac{-\boldsymbol{C}_{1 i} \boldsymbol{C}_{2 i}^{\top}}{P_{i}^{2}}+\frac{\boldsymbol{H}_{2 i}}{P_{i}}\right] \boldsymbol{X}_{2 i}, \\
& \frac{\partial^{2} l(\boldsymbol{\theta})}{\partial \tau_{1} \partial \boldsymbol{\beta}_{1}}=\sum_{i=1}^{N} \boldsymbol{X}_{1 i}^{\top}\left[\frac{-\boldsymbol{C}_{1 i} C_{3 i}}{P_{i}^{2}}+\frac{\boldsymbol{H}_{3 i}}{P_{i}}\right], \\
& \frac{\partial^{2} l(\boldsymbol{\theta})}{\partial \tau_{2} \partial \boldsymbol{\beta}_{1}}=\sum_{i=1}^{N} \boldsymbol{X}_{1 i}^{\top}\left[\frac{-\boldsymbol{C}_{1 i} C_{4 i}}{P_{i}^{2}}+\frac{\boldsymbol{H}_{4 i}}{P_{i}}\right] \\
& \frac{\partial^{2} l(\boldsymbol{\theta})}{\partial \boldsymbol{\beta}_{2}^{2}}=\sum_{i=1}^{N} \boldsymbol{X}_{2 i}^{\top}\left[\frac{-\boldsymbol{C}_{2 i} \boldsymbol{C}_{2 i}^{\top}}{P_{i}^{2}}+\frac{\boldsymbol{H}_{5 i}}{P_{i}}\right] \boldsymbol{X}_{2 i}, \\
& \frac{\partial^{2} l(\boldsymbol{\theta})}{\partial \tau_{1} \partial \boldsymbol{\beta}_{2}}=\sum_{i=1}^{N} \boldsymbol{X}_{2 i}^{\top}\left[\frac{-\boldsymbol{C}_{2 i} C_{3 i}}{P_{i}^{2}}+\frac{\boldsymbol{H}_{6 i}}{P_{i}}\right] \\
& \frac{\partial^{2} l(\boldsymbol{\theta})}{\partial \tau_{2} \partial \boldsymbol{\beta}_{2}}=\sum_{i=1}^{N} \boldsymbol{X}_{2 i}^{\top}\left[\frac{-\boldsymbol{C}_{2 i} C_{4 i}}{P_{i}^{2}}+\frac{\boldsymbol{H}_{7 i}}{P_{i}}\right] \\
& \frac{\partial^{2} l(\boldsymbol{\theta})}{\partial \tau_{1}^{2}}=\sum_{i=1}^{N}\left[\frac{-C_{3 i}^{2}}{P_{i}^{2}}+\frac{H_{8 i}}{P_{i}}\right] \\
& \frac{\partial^{2} l(\boldsymbol{\theta})}{\partial \tau_{1} \partial \tau_{2}}=\sum_{i=1}^{N}\left[\frac{-C_{3 i} C_{4 i}}{P_{i}^{2}}+\frac{H_{9 i}}{P_{i}}\right] \\
& \sum_{i=1}^{N}\left[\frac{-C_{4 i}^{2}}{P_{i}^{2}}+\frac{H_{10 i}}{P_{i}}\right] .
\end{aligned}
$$

O esboço do procedimento para obter as componentes da função escore e da matriz hessiana é apresentado no Apêndice C.

A estimativa $\hat{\boldsymbol{\theta}}$ para o vetor de parâmetros $\boldsymbol{\theta}$ é obtida maximizando a função de log-verossimilhança $l(\boldsymbol{\theta})$ da expressão (3.14) no espaço paramétrico $\boldsymbol{\Theta}$. Neste trabalho foi construído um pacote específico para análise do modelo proposto e denominado 'wlmm' em linguagem R, o qual dispõe da função wlmmML para obter as estimativas dos parâmetros do modelo. Uma descrição das principais funções do pacote pode ser encontrada no Capítulo 8.

\subsection{Predição dos efeitos aleatórios}

Predições dos efeitos aleatórios são necessárias sempre que o interesse seja a previsão para os grupos e a validação das suposições do modelo (Molenberghs e Verbeke, 2005). A predição dos efeitos aleatórios pode estar baseada no método chamado de melhor predição (Best Prediction) que tem a característica de o valor esperado da predição do efeito aleatório 
coincidir com o valor esperado do efeito aleatório. Para mais detalhes, vide, por exemplo, Searle et al. (2006) e McCulloch et al. (2008). A predição dos efeitos aleatórios $u_{1 i}$ e $u_{2 i}$ é denotada por $\tilde{u}_{1 i}$ e $\tilde{u}_{2 i}$, respectivamente. As expressões para a obtenção das predições são

$$
\begin{aligned}
\tilde{u}_{1 i} & =E\left(u_{1 i} \mid \boldsymbol{y}_{i}\right)=\frac{\iint_{\Re^{2}} u_{1 i} P\left(\boldsymbol{y}_{i}, \boldsymbol{u}_{i}\right) d \boldsymbol{u}_{i}}{\iint_{\Re^{2}} P\left(\boldsymbol{y}_{i}, \boldsymbol{u}_{i}\right) d \boldsymbol{u}_{i}} \\
\text { e } \quad \tilde{u}_{2 i}=E\left(u_{2 i} \mid \boldsymbol{y}_{i}\right) & =\frac{\iint_{\Re^{2}} u_{2 i} P\left(\boldsymbol{y}_{i}, \boldsymbol{u}_{i}\right) d \boldsymbol{u}_{i}}{\iint_{\Re^{2}} P\left(\boldsymbol{y}_{i}, \boldsymbol{u}_{i}\right) d \boldsymbol{u}_{i}} .
\end{aligned}
$$

Os preditores $\tilde{u}_{1 i}$ e $\tilde{u}_{2 i}$ são também chamados de estimadores de Bayes empíricos (McCulloch et al., 2008). Note que as expressões (3.15) e (3.16) podem ser calculadas a partir da expressão (3.13) e a integração pode ser realizada usando QGH.

\subsection{Proposta de Resíduos}

A análise dos resíduos é uma forma eficiente de detectar deficiências em relação ao modelo ajustado, tais como a presença de observações discrepantes, a ausência de componentes na parte sistemática do modelo, afastamentos das suposições dos erros e da variância. Neste trabalho propõe-se o uso de três tipos de resíduos, o resíduo ordinário padronizado, o resíduo componente do desvio e o resíduo quantil normal aleatorizado usado nos GAMLSS.

\subsubsection{Resíduo Ordinário Padronizado}

Uma forma simples para definir o resíduo ordinário padronizado $\left(r_{o}\right)$ que é dado pela expressão

$$
r_{o_{i j}}=\frac{y_{i j}-\hat{E}\left(y_{i j}\right)}{\sqrt{\widehat{\operatorname{Var}}\left(y_{i j}\right)}}
$$

em que $\hat{E}\left(y_{i j}\right)$ e $\widehat{\operatorname{Var}}\left(y_{i j}\right)$ correspondem à média e variância estimadas para a observação $i j$. Este resíduo pode ser obtido para o modelo estudado e depende do vetor de estimativas $\hat{\boldsymbol{\theta}}$ e das predições dos efeitos aleatórios.

\subsubsection{Resíduo Componente do Desvio}

Therneau et al. (1990) propuseram os resíduos componente do desvio $\left(r_{d}\right)$ baseados em resíduos martingales e foram usados em modelos de regressão log-gama generalizados por Ortega et al. (2008), modelos log-Birnbaum-Saunders mistos por Villegas et al. (2011) e em modelos de regressão Beta-Weibull por Ortega et al. (2011). O resíduo componente do desvio para a $j$-ésima observação do grupo $i$ é dado por

$$
r_{d_{i j}}=\operatorname{sinal}\left(r_{M_{i j}}\right) \sqrt{-2\left[r_{M_{i j}}+\delta_{i j} \log \left(\delta_{i j}-r_{M_{i j}}\right)\right]}
$$


em que $r_{M_{i j}}$ é o resíduo martingal o qual é assimétrico, assume valor máximo em +1 e valor mínimo em $-\infty . \delta_{i j}=0$ se a observação é censurada e o valor 1 caso contrário (Ortega et al., 2011). O resíduo $r_{M_{i j}}$ é dado pela expressão

$$
r_{M_{i j}}=\delta_{i j}+\log \left[S\left(y_{i j} ; \hat{\boldsymbol{\theta}}\right)\right]
$$

em que $S(\cdot)$ corresponde à função de sobrevivência dada na expressão (3.3). O resíduo pode ser obtido para o modelo estudado e depende do vetor de estimativas $\hat{\boldsymbol{\theta}}$ e das predições dos efeitos aleatórios.

\subsubsection{Resíduo Quantil Normal Aleatorizado}

O resíduo quantil normal aleatorizado $\left(r_{q}\right)$ foi proposto por Dunn e Smyth (1996) para modelos de regressão com respostas independentes do tipo contínuo ou discreto e Rigby e Stasinopoulos (2005) empregam esse tipo de resíduos nos GAMLSS. Esses resíduos têm distribuição normal, pois sua construção é feita invertendo a função de distribuição ajustada para cada resposta e procurando o quantil equivalente na distribuição normal padrão. $\mathrm{O}$ resíduo para um modelo de regressão com distribuição contínua para a variável resposta é dado por

$$
r_{q_{i j}}=\Phi^{-1}\left(F\left(y_{i j} \mid \hat{\boldsymbol{\theta}}\right)\right)
$$

em que $\Phi^{-1}$ corresponde à função quantil da normal padrão e $F(\cdot)$ é uma função de distribuição acumulada, como por exemplo a dada na expressão (3.2). Os resíduos quantil normal aleatorizados têm distribuição normal padrão se o modelo for definido corretamente (Rigby e Stasinopoulos, 2005).

Note-se que as distribuições dos resíduos propostos $r_{o}$ e $r_{d}$ são desconhecidas e é difícil concluir analíticamente sobre as mesmas. Por tal razão foi realizado um estudo de simulação de Monte Carlo e os resultados são apresentados na Seção 3.7.

\subsection{Teste sobre as componentes de variância}

No modelo aqui estudado é importante verificar se os parâmetros $\tau_{1}$ e $\tau_{2}$, associados à variabilidade dos efeitos aleatórios, são significativos para decidir se é correto incluir interceptos aleatórios ou não nos modelos associados aos parâmetros $\mu$ e $\sigma$ da distribuição. A hipótese nula nesse tipo de problemas está na fronteira do espaço paramétrico $\left(\Re_{+}^{2}\right)$. Portanto, é preciso usar uma ferramenta que leve isso em consideração. Uma forma de abordar essa situação é usando o teste da razão de verossimilhanças (RV).

Shapiro (1988) e Stram e Lee (1994, 1995) obtiveram que a distribuição assintótica da estatística do teste RV é uma mistura de distribuições qui-quadrado para modelos lineares 
normais mistos sob a suposição de que as respostas são independentes e identicamente distribuídas; Vu e Zhou (1997) estenderam o estudo para o caso em que as observações não são identicamente distribuídas. No entanto, Crainiceanu e Ruppert (2004) afirmaram que, quando assumida uma mistura de distribuições qui-quadrado para a estatística do teste RV, obtém-se um desempenho pobre do teste para tamanhos de amostras moderados. Giampaoli e Singer (2009) usaram os resultados menos restritivos de Vu e Zhou (1997) para provar que a mistura de distribuições qui-quadrado é a distribuição assintótica do teste RV usado como estatística de teste para componentes de variância em modelos com um ou dois efeitos aleatórios correlacionados. Villegas (2010) e Villegas et al. (2011) usaram o teste RV para testar a inclusão do intercepto aleatório em modelos log-Birnbaum-Sanders mistos com observações censuradas.

Na subseção seguinte apresenta-se o teste RV para o modelo proposto. Como a distribuição da estatística do teste é desconhecida, na seção 3.7 apresentam-se os resultados de um estudo de simulação Monte Carlo no qual foi estudada a distribuição empírica da estatística do teste RV.

\subsubsection{Teste da razão de verossimilhanças}

Para o modelo (3.4) tem-se a suposição de que os efeitos aleatórios $u_{1 i}$ e $u_{2 i}$ têm distribuição normal de média zero e variâncias $\tau_{1}^{2}$ e $\tau_{2}^{2}$, respectivamente. Para aplicação do teste da razão de verossimilhanças no modelo estudado é importante identificar os seguintes três casos.

\section{Caso 1}

Este corresponde ao caso principal no qual é testado o seguinte conjunto de hipóteses:

$$
H_{0}:\left(\begin{array}{c}
\tau_{1} \\
\tau_{2}
\end{array}\right)=\mathbf{0} \quad \text { contra } \quad H_{A}:\left(\begin{array}{c}
\tau_{1} \\
\tau_{2}
\end{array}\right) \neq \mathbf{0}
$$

Para testar as hipóteses dois modelos são ajustados, o primeiro deles sob $H_{0}$ e que corresponde ao modelo sem interceptos aleatórios para os parâmetros $\mu$ e $\sigma$. O segundo modelo ajustado supõe interceptos aleatórios descorrelacionados. Sejam $\hat{\boldsymbol{\theta}}_{H_{0}}$ e $\hat{\boldsymbol{\theta}}$ o vetor de estimadores sob $H_{0}$ e $H_{A}$, respectivamente. A estatística para o teste é dada pela expressão

$$
\xi_{R V}=2\left[l(\hat{\boldsymbol{\theta}})-l\left(\hat{\boldsymbol{\theta}}_{H_{0}}\right)\right]
$$

Caso a hipótese nula seja rejeitada é importante saber se as componentes $\tau_{1}$ ou $\tau_{2}$ são diferentes de zero. Os dois casos seguintes têm como objetivo estudar essa situação. 


\section{Caso 2}

Neste caso o interesse é testar

$$
H_{0}: \tau_{1}=0 \text { contra } H_{A}: \tau_{1}>0
$$

\section{Caso 3}

Neste caso o interesse é testar

$$
H_{0}: \tau_{2}=0 \text { contra } H_{A}: \tau_{2}>0
$$

A distribuição empírica das estatísticas nos modelos propostos é explorada por meio de um estudo de simulação apresentado na seguinte seção.

\subsection{Estudo de simulação}

Nesta seção são apresentados os resultados de um estudo de simulação Monte Carlo que foi realizado com o intuito de analisar o modelo proposto, em particular, examinar o desempenho do processo de estimação dos parâmetros, a distribuição das componentes de $\hat{\boldsymbol{\theta}}$, a distribuição empírica dos resíduos propostos, assim como a distribuição empírica da estatística e o comportamento do teste da razão de verossimilhanças. A estrutura do modelo usada no estudo para as simulações foi a seguinte:

$$
\begin{aligned}
y_{i j} \mid u_{1 i}, u_{2 i} & \stackrel{i n d}{\sim} W E I 3\left(\mu_{i j}, \sigma_{i j}\right), \\
u_{1 i} & \sim N\left(0 ; 0,8^{2}\right), \\
u_{2 i} & \sim N\left(0 ; 0,9^{2}\right), \\
\log \left(\mu_{i j}\right) & =-1+0,5 x_{1}+u_{1 i} \\
\text { e } \quad \log \left(\sigma_{i j}\right) & =1,3+0,3 x_{2}+u_{2 i}, \quad i=1,2, \ldots, N, \quad j=1,2, \ldots, n_{i},
\end{aligned}
$$

em que $x_{1} \sim N\left(2 ; 0,3^{2}\right)$ e $x_{2} \sim N\left(-1 ; 0,3^{2}\right)$. Assim, tem-se que o vetor de parâmetros $\boldsymbol{\theta}$ a estimar tem como valores verdadeiros $\boldsymbol{\theta}=(-1 ; 0,5 ; 1,3 ; 0,3 ; 0,8 ; 0,9)^{\top}$. Simularam-se 10000 amostras com a estrutura (3.22) para cada cenário resultante da combinação de $N=5$, 10, 25 e 50 número de grupos, $n_{i}=3,5,10$ e 15 observações por grupo e percentagem de observações censuradas de 0\%,20\% e 40\%. Por exemplo, para a geração das observações censuradas com 20\%, foi calculado o quantil $20 \%$ e aquelas observações maiores o iguais ao quantil foram substituídas pelo valor do quantil. Para cada uma das amostras foi ajustado o modelo obtendo-se e armazenando-se o vetor de estimativas $\hat{\boldsymbol{\theta}}$ e os resíduos $r_{o}, r_{q}, r_{M}$ e $r_{d}$. 


\subsubsection{Processo de estimação}

Para estudar o desempenho do processo de estimação foi usado o erro quadrático médio multivariado (EQMM) apresentado por Wissel (2009) e definido como

$$
E Q M M=(\hat{\boldsymbol{\theta}}-\boldsymbol{\theta})^{\top}(\hat{\boldsymbol{\theta}}-\boldsymbol{\theta})+\operatorname{tr}(\Sigma(\hat{\boldsymbol{\theta}})),
$$

o qual leva em conta a distância entre $\hat{\boldsymbol{\theta}}$ e $\boldsymbol{\theta}$ assim como a variabilidade $\Sigma$ do vetor de estimativas.

Na Tabela 3.1 apresentam-se as médias do EQMM para cada combinação de $N, n_{i}$ e diferentes percentagens de censura. Observa-se que à medida que $N$ ou $n_{i}$ crescem, o EQMM diminui, o que significa que as estimativas $\hat{\boldsymbol{\theta}}$ ficam mais próximas de $\boldsymbol{\theta}$ à medida que há maior número de observações disponíveis. Em geral, observa-se também que à medida que aumenta a percentagem de observações censuradas o EQMM aumenta.

Tabela 3.1: EQMM entre $\hat{\boldsymbol{\theta}}$ e $\boldsymbol{\theta}$ no estudo de simulação para o modelo Weibull normal.

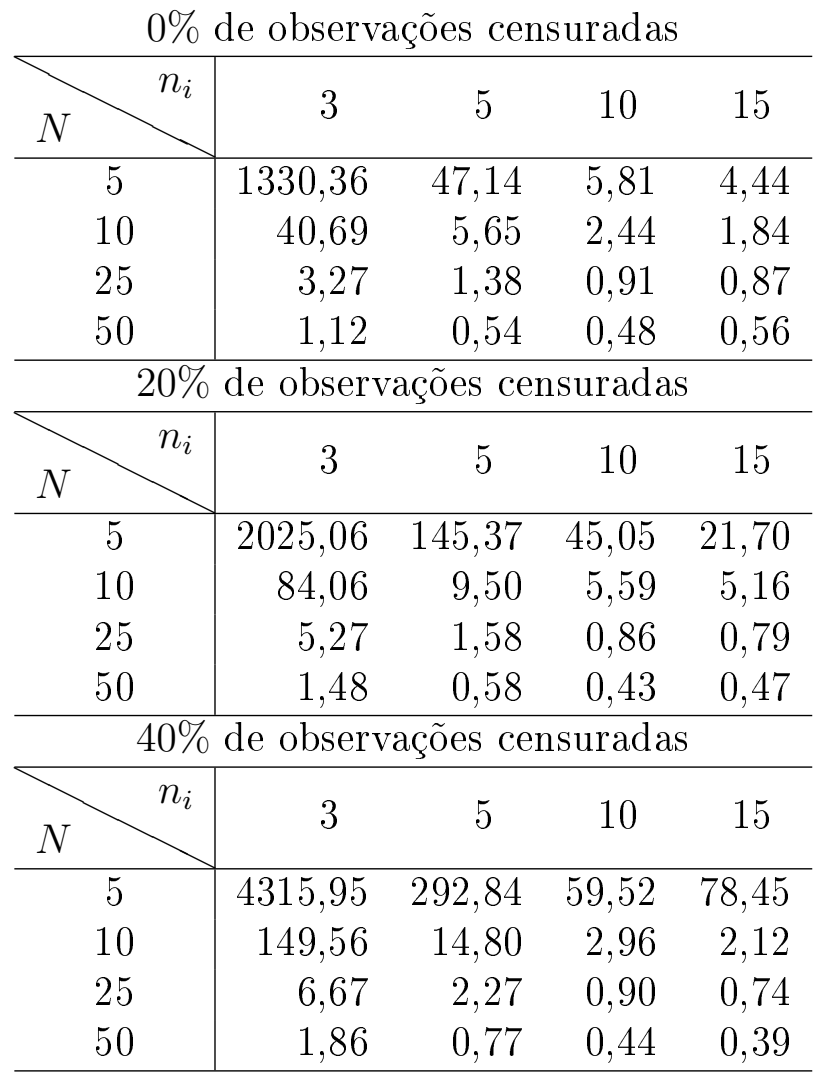

\subsubsection{Distribuição empírica dos estimadores}

Para analisar a distribuição empírica das componentes de $\hat{\boldsymbol{\theta}}$ foi construída a Figura 3.2. Nessa figura tem-se as densidades para cada uma das componentes do vetor $\hat{\boldsymbol{\theta}}$ em dois casos 
extremos, o primeiro com número de observações pequeno, $N=10$ e $n_{i}=3$, e o segundo com número de observações maior, $N=50$ e $n_{i}=10$. Da figura observa-se que as distribuições para as componentes $\hat{\beta}_{11}, \hat{\beta}_{12}, \hat{\beta}_{21}, \hat{\beta}_{22}$ e $\hat{\tau}_{1}$ são simétricas e centradas no verdadeiro valor dado pela linha vertical tracejada escura. Em relação a $\hat{\tau}_{2}$, tem-se que a moda da distribuição ficou próxima do verdadeiro valor dado pela linha vertical. Observa-se também que para o caso com maior quantidade de dados, $N=50$ e $n_{i}=10$, a distribuição empírica de cada componente ficou mais concentrada ao redor do verdadeiro valor.
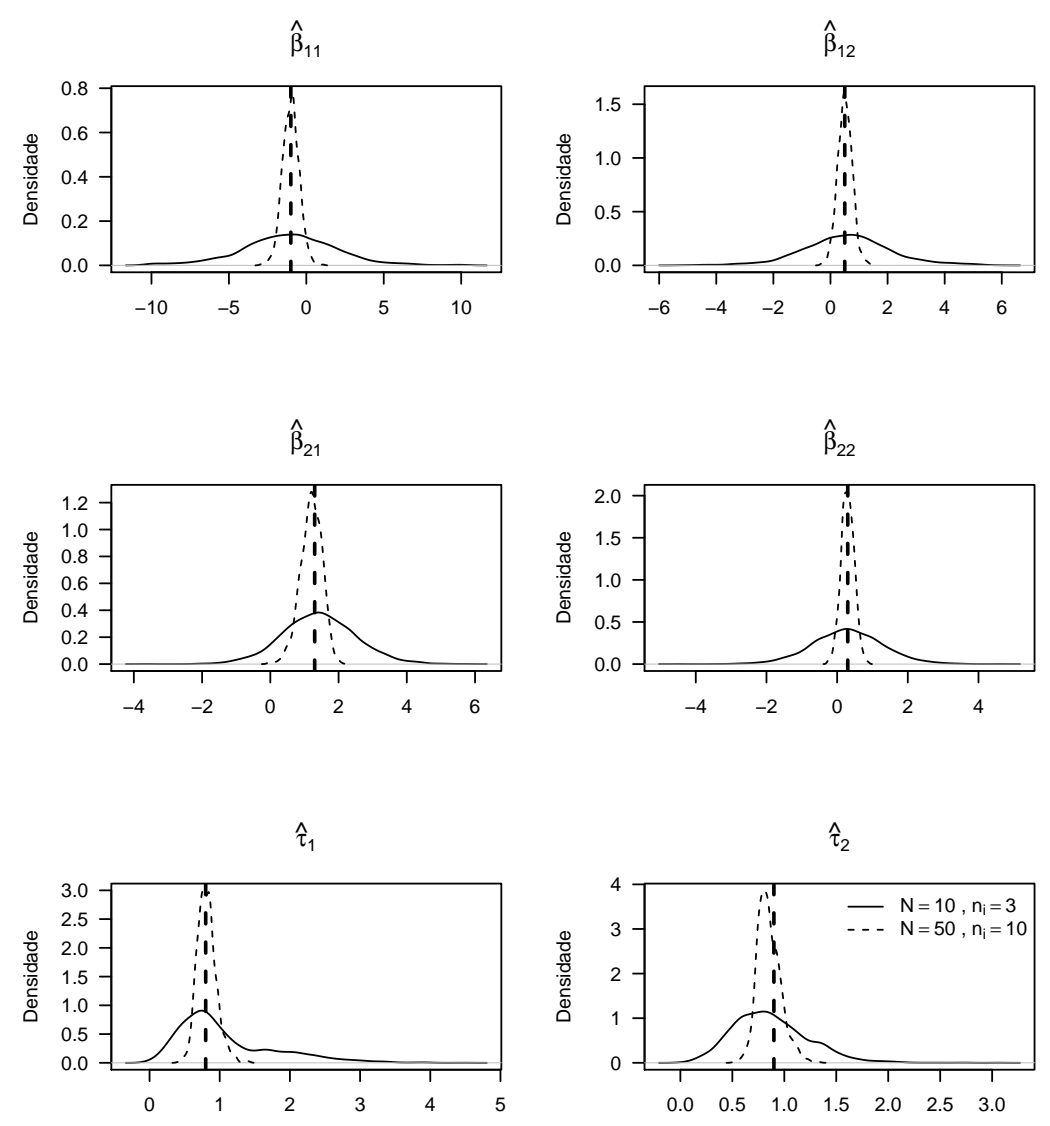

Figura 3.2: Função densidade estimada para as componentes de $\hat{\boldsymbol{\theta}}$ no modelo Weibull normal. A linha continua corresponde ao caso de $N=10$ e $n_{i}=3$. A linha tracejada corresponde ao caso de $N=50$ e $n_{i}=10$. A linha tracejada vertical escura corresponde a cada um dos valores verdadeiros de $\boldsymbol{\theta}$.

\subsubsection{Distribuição empírica dos resíduos}

Para estudar a distribuição empírica dos resíduos propostos, os quantis dos resíduos foram graficados contra os quantis da distribuição normal padrão para algumas combinações de $N, n_{i}$; os resultados são apresentados nas Figuras 3.3 a 3.10 e pode-se concluir o seguinte:

- Das Figuras 3.3 e 3.4 observa-se que a distribuição para $r_{o}$ não é normal devido a que os pontos associados não ficaram na reta identidade. Além disso, foram observados 
resíduos com valores absolutos superiores a 3 quando $n_{i}=3$ e $40 \%$ de censura.

- Das Figuras 3.5 e 3.6 nota-se que a distribuicão de $r_{q}$, no caso de $0 \%$ e $20 \%$ de censura, ficaram perto da reta identidade à medida que aumenta $n_{i}$. No entanto, no caso de $40 \%$ de censura observa-se que os pontos não ficaram na reta identidade o qual é mais evidente para $n_{i}=3$.

- Nas Figuras 3.7 e 3.8 observa-se que os pontos no caso sem censura ficaram na reta identidade, no entanto, o aumento na censura provocou uma curvatura em torno de $r_{d}=0$, esta situação é evidente no caso de $n_{i}=15$.

- Como esperado, das Figuras 3.9 e 3.10 observa-se que a distribuição para $r_{M}$ é assimétrica com valor máximo em 1 .
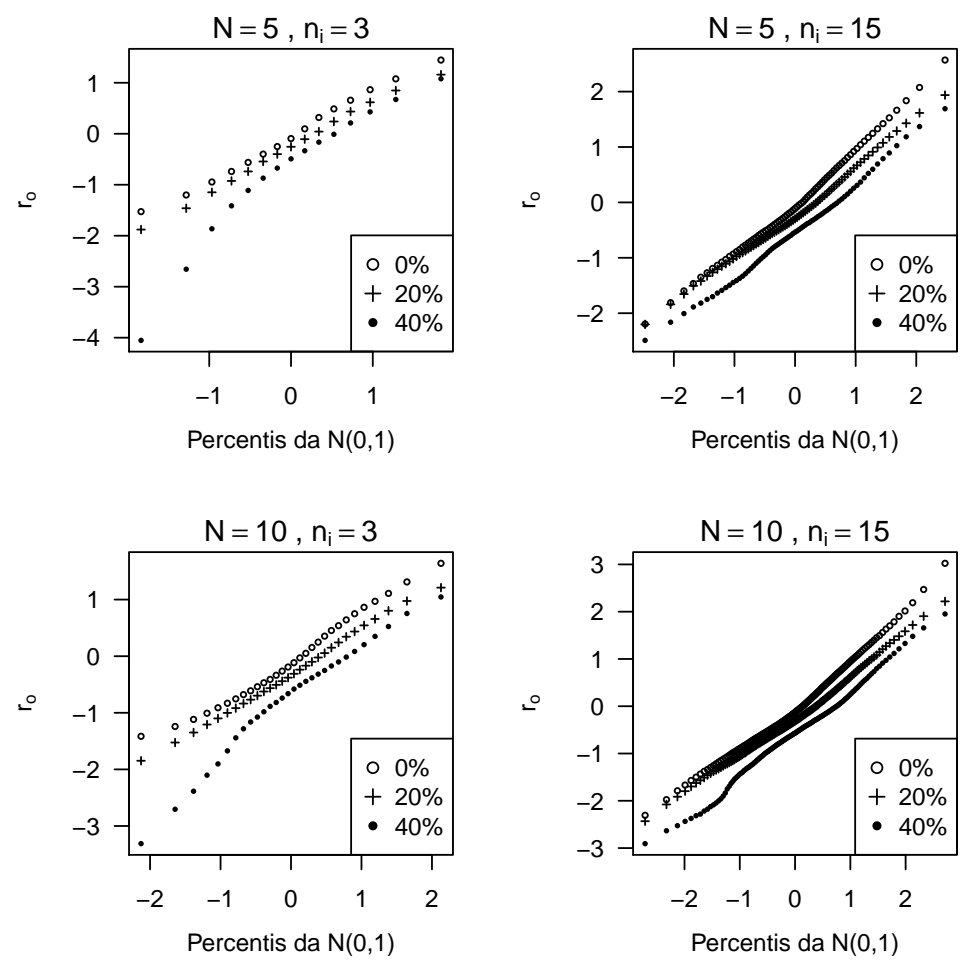

Figura 3.3: Gráfico normal de probabilidade para o resíduo $r_{o}$ no modelo Weibull normal com diferentes percentagens de censura para $N=5$ e $N=10$.

As medidas de média, desvio padrão, coeficiente de curtorse e coeficiente de asimetria foram obtidas para os resíduos $r_{o}, r_{q}$ e $r_{d}$, baseadas nas 10000 simulações, para estudar melhor a distribuição empirica e os resultados são apresentados nas Tabelas 3.2 a 3.4. Na Tabela 3.2, que corresponde ao resíduo $r_{o}$ destaca-se que a média tende a se afastar de 0 à medida que aumenta a percentagem de censura, enquanto o desvio padrão e a curtose mantêm-se ao redor de 0,85 e 2,87, respectivamente. Comportamento semelhante em relação à média foi observado a partir da Tabela 3.3 para $r_{q}$, com desvio padrão e a curtose ao redor de 0,90 e 2,48, respectivamente. Na Tabela 3.4 observa-se que a média de $r_{d}$ tende a 

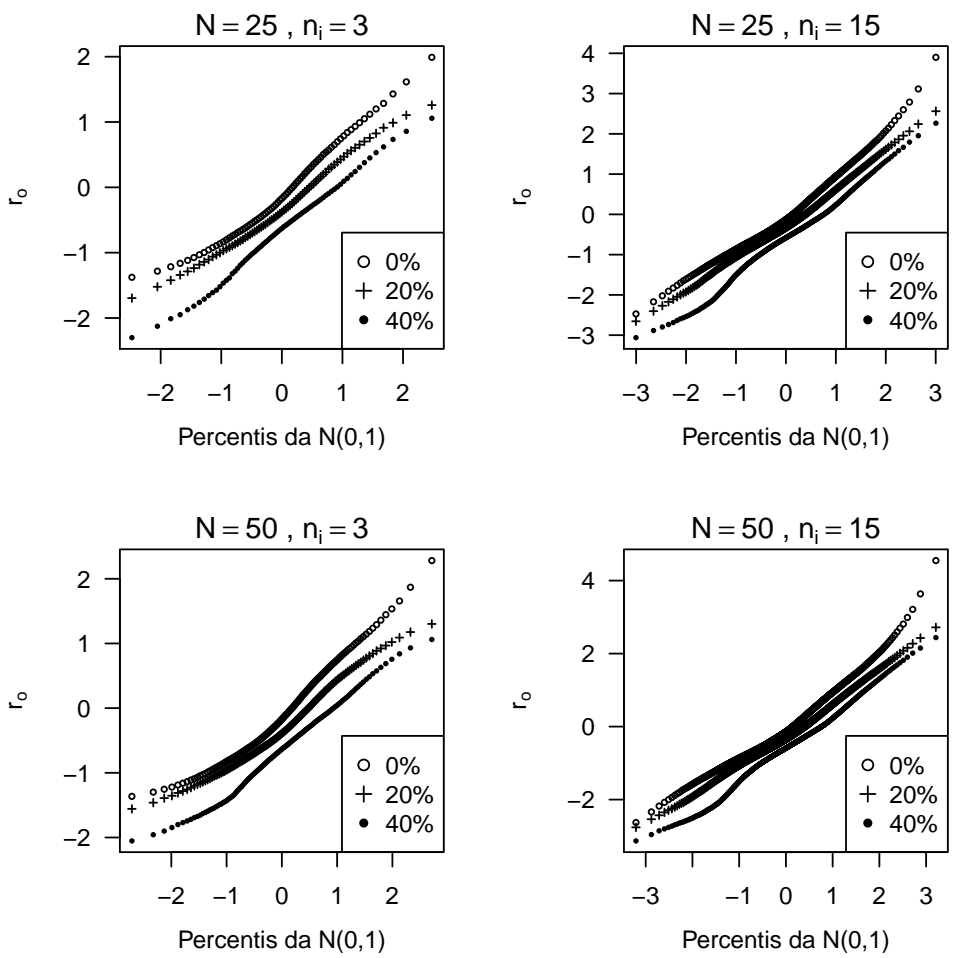

Figura 3.4: Gráfico normal de probabilidade para o resíduo $r_{o}$ no modelo Weibull normal com diferentes percentagens de censura para $N=25$ e $N=50$.

zero à medida que a percentagem de censura aumenta, enquanto o desvio padrão e a curtose para $r_{o}$ permaneceram constantes em torno de 0,90 e 2,34, respectivamente. Adicionalmente, observou-se que o coeficiente de assimetria ficou ao redor de 0 só para $r_{q}$ e $r_{d}$.

Em geral, a partir das tabelas, nota-se que a distribuição empírica para os resíduos $r_{q} \mathrm{e}$ $r_{d}$ é caracterizada por valores de desvio padrão, curtose e assimetria em torno de 0,90, 2,41 e 0 , respectivamente. Além disso, a média tende a se afastar de 0 para $r_{o}$ e $r_{q}$ e o oposto ocorre para $r_{d}$ à medida que a percentagem de censura aumenta. Foi aplicado o teste de ShapiroWilk para os resíduos em cada combinação de $N, n_{i}$ e percentagem de censura. Verificou-se que a taxa de rejeição da hipótese de normalidade, baseada nas 10000 simulações, foi 29\%, $4 \%$ e $15 \%$ para $r_{o}, r_{q}$ e $r_{d}$, respectivamente. A partir desses resultados, pode-se concluir que as distribuições empíricas para $r_{q}$ e $r_{d}$ são próximas da distribuição normal e assim esses resíduos podem ser usados facilmente para verificar se o modelo ajustado está corretamente especificado.

\subsubsection{Distribuição empírica da estatística da RV}

Para estudar a distribuição empírica da estatística e o comportamento do teste RV proposto na Seção 3.6.1, foram conduzidas novas simulações segundo a estrutura em (3.22). Foram mantidos fixos os vetores de efeitos $\boldsymbol{\beta}_{1}$ e $\boldsymbol{\beta}_{2}$. Os valores considerados para o número de grupos e observações por grupo foram $N=5,10,25,50$ e $n_{i}=3,5,10$ e 15 

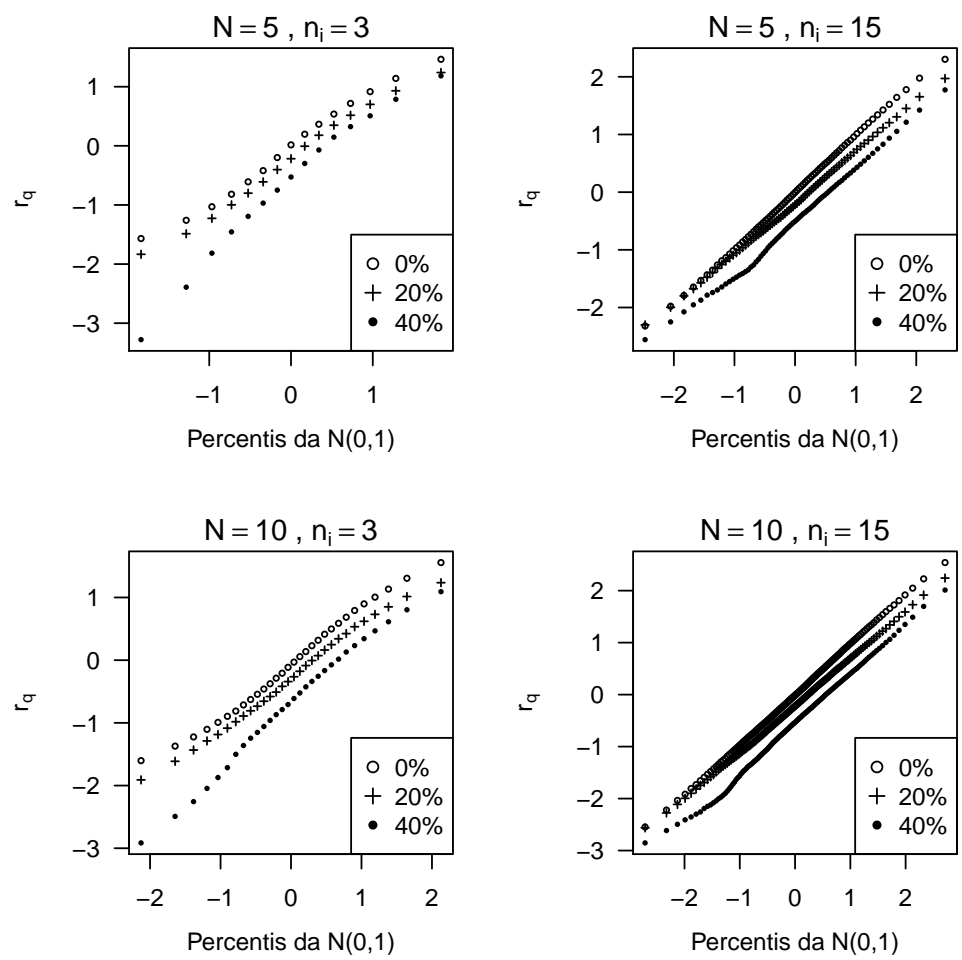

Figura 3.5: Gráfico normal de probabilidade para o resíduo $r_{q}$ no modelo Weibull normal com diferentes percentagens de censura para $N=5$ e $N=10$.

respectivamente. As componentes $\tau_{1}$ e $\tau_{2}$, associadas às variabilidades dos efeitos aleatórios, assumiram valores $0,0.5$, e 1 . O interesse nessa parte do estudo de simulação foi testar o seguinte conjunto de hipóteses:

$$
H_{0}:\left(\begin{array}{c}
\tau_{1} \\
\tau_{2}
\end{array}\right)=\mathbf{0} \quad \text { contra } \quad H_{A}:\left(\begin{array}{c}
\tau_{1} \\
\tau_{2}
\end{array}\right) \neq \mathbf{0}
$$

Para estudar a distribuição empírica da estatística $\xi_{R V}$, sob $H_{0}$ verdadeira, foram geradas 1000 amostras para cada cenário resultante da combinação de $N, n_{i} \operatorname{com} \tau_{1}=0$ e $\tau_{2}=0$ segundo a estrutura (3.22). Para cada uma das amostras simuladas foi calculada a estatística $\xi_{R V}$ dada na expressão (3.21) e construído o gráfico de probabilidade das estatísticas $\xi_{R V}$ obtidas versus os percentis da $\chi_{0: 1}^{2}$ (mistura meio a medio de duas $\chi^{2}$ com 0 e 1 graus de liberdade), que é a distribuição candidata para a estatística sob $H_{0}$ verdadeira. Nas Figuras 3.11 a 3.14 têm-se os diferentes gráficos obtidos e deles observa-se que os pontos em cada um dos paniéis ficaram próximos da linha a 45 graus, o que indica que a distribuição da estatística $\xi_{R V}$, sob $H_{0}$ verdadeira, pode ser considerada como uma combinação 0,5:0,5 de $\chi_{0}^{2}$ e $\chi_{1}^{2}$. Observa-se também que para $N=5$ e $N=10$ com 3 observações por grupo, a distribuição da estatística $\xi_{R V}$ se afasta da distribuição esperada devido ao baixo número de observações.

Para estudar o desempenho do teste RV foram geradas 1000 amostras para cada cenário resultante da combinação de $N, n_{i}, \tau_{1}$ e $\tau_{2}$ segundo a estrutura (3.22). Para cada uma das 

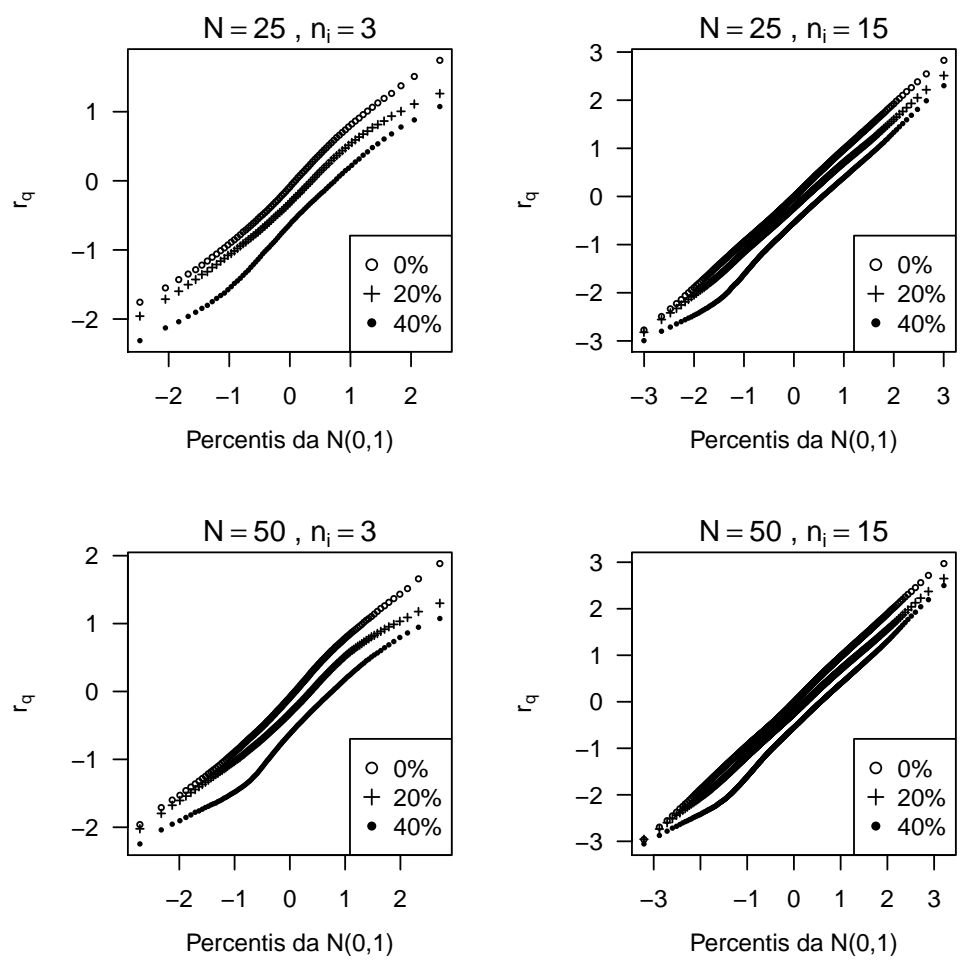

Figura 3.6: Gráfico normal de probabilidade para o resíduo $r_{q}$ no modelo Weibull normal com diferentes percentagens de censura para $N=25$ e $N=50$.

amostras simuladas foi calculada a estatística $\xi_{R V}$ dada na expressão (3.21) e testada $H_{0}$ a um nível de significância de $5 \%$ assumindo que $\xi_{R V} \sim 0.5 \chi_{0}^{2}+0.5 \chi_{1}^{2}$. Posteriormente, foi obtida a porcentagem de vezes em que $H_{0}$ foi rejeitada em de cada cenário. Para $\tau_{1}=0$ e $\tau_{2}=0$, sob a hipótese nula verdadeira, é esperada uma taxa de rejeições ao redor de $5 \%$. Para qualquer outra combinação de $\tau_{1}$ e $\tau_{2}$ é esperado que a taxa de rejeições aumente dado que a hipótese nula é falsa.

Os resultados obtidos ao testar $H_{0}$ são apresentados na Tabela 3.5. Da tabela observa-se que no caso de $\tau_{1}=\tau_{2}=0$, as taxas de rejeições ficaram em torno de $5 \%$ para $N=5,10$. Quando $N=25$ e $N=50$ essa taxa foi só um pouco superior ao valor esperado de $5 \%$. Para as outras combinações de $\tau_{1}$ e $\tau_{2}$ observou-se um padrão de aumento na taxa de rejeições na medida em que $N$ e $n_{i}$ aumentaram. Em resumo, observou-se que quando $H_{0}$ foi verdadeira a taxa de rejeições de $H_{0}$ ficou em torno de $5 \%$, enquanto para $H_{0}$ falsa a taxa aumentou.

\subsection{Aplicações}

Nesta seção apresentam-se duas aplicações do método desenvolvido nesse capítulo. A primeira aplicação é realizada usando uma base de dados simulada na qual as componentes do vetor de estimativas $\boldsymbol{\theta}$ são conhecidas. Na segunda, usa-se uma base de dados real relacionada 

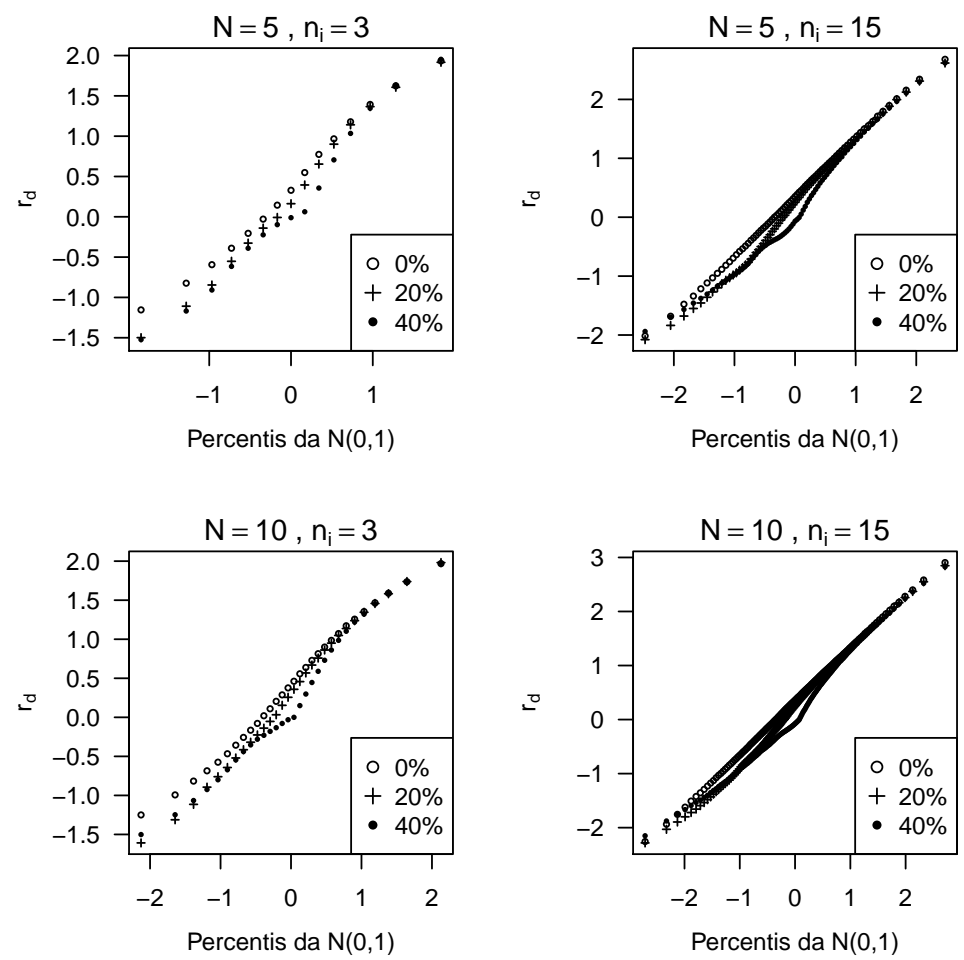

Figura 3.7: Gráfico normal de probabilidade para o resíduo $r_{d}$ no modelo Weibull normal com diferentes percentagens de censura para $N=5$ e $N=10$.

aos tempos necessários para que pacientes com leucemia obtenham um transplante de medula óssea.

\subsubsection{Exemplo com base de dados simulada}

Nesta seção apresenta-se um exemplo da estimação no modelo com resposta Weibull, sem censura e com efeitos aleatórios normais nos modelos dos parâmetros $\mu$ e $\sigma$. A base de dados contém 50 observações divididas em $N=10$ grupos, e $n_{i}$ número de observações por grupo igual a 5. Para cada observação tem-se a variável resposta $y$ e duas variáveis explicativas $x_{1}$ e $x_{2}$. O modelo usado para a geração dos dados foi o seguinte:

$$
\begin{aligned}
y_{i j} \mid u_{1 i}, u_{2 i} & \stackrel{i n d}{\sim} W \operatorname{EI}\left(\mu_{i j}, \sigma_{i j}\right), \\
\log \left(\mu_{i j}\right) & =-1+0.5 x_{1}+u_{1 i} \\
\text { e } \quad \log \left(\sigma_{i j}\right) & =1.3+0.3 x_{2}+u_{2 i}, \quad i=1,2, \ldots, 10 \quad j=1,2, \ldots, 5
\end{aligned}
$$

em que $u_{1 i} \sim N\left(0 ; 0,8^{2}\right), u_{2 i} \sim N\left(0 ; 1^{2}\right), x_{1} \sim N\left(2 ; 0,3^{2}\right)$ e $x_{2} \sim N\left(-1 ; 0,3^{2}\right)$. Assim, tem-se que o vetor de parâmetros é $\boldsymbol{\theta}=(-1 ; 0,5 ; 1,3 ; 0,3 ; 0,8 ; 1)^{\top}$. Os dados são apresentados na Tabela B.1 do Apêndice B. Na Figura 3.15 apresenta-se a densidade para a variável $y$ da base de dados simulada e observa-se que os valores y são inferiores ao valor de 2 .

No ajuste do modelo foi usada uma aproximação QGH com 10 pontos e a obtenção 



Figura 3.8: Gráfico normal de probabilidade para o residuo $r_{d}$ no modelo Weibull normal com diferentes percentagens de censura para $N=25$ e $N=50$.

dos parâmetros foi feita por meio da função nlminb em R. Com o intuito de comparar as estimativas obtidas, foi ajustado o mesmo modelo com o pacote GAMLSS e os resultados dos ajustes são apresentados na Tabela 3.6. Na tabela observa-se que as estimativas dos efeitos fixos obtidas com o método proposto e com GAMLSS são próximas e observa-se também que ficaram próximos dos valores verdadeiros para $\boldsymbol{\theta}$. Na implementação dos GAMLSS em R não é possível obter estimativas para os erro padrão das componentes de variância.

Uma das suposições do modelo estudado é que os efeitos aleatórios têm distribuição normal, portanto construiu-se o gráfico normal de probabilidade para cada um dos efeitos aleatórios preditos. Na Figura 3.16 temos os gráficos normais para os efeitos aleatórios preditos com os valores-p do teste de normalidade Anderson-Darling (D'Agostinho e Stephens, 1986). Os resultados obtidos indicam que não é rejeitada a hipótese de normalidade dos efeitos aleatórios. Os três resíduos, $r_{o}, r_{d}$ e $r_{q}$ foram obtidos para o exemplo e na Figura 3.17 encontramos os gráficos normais para cada um dos resíduos. Junto aos gráficos normais aparecem os valores-p do teste Anderson-Darling de normalidade. Os resultados indicam que a hipótese de normalidade não é rejeitada. 

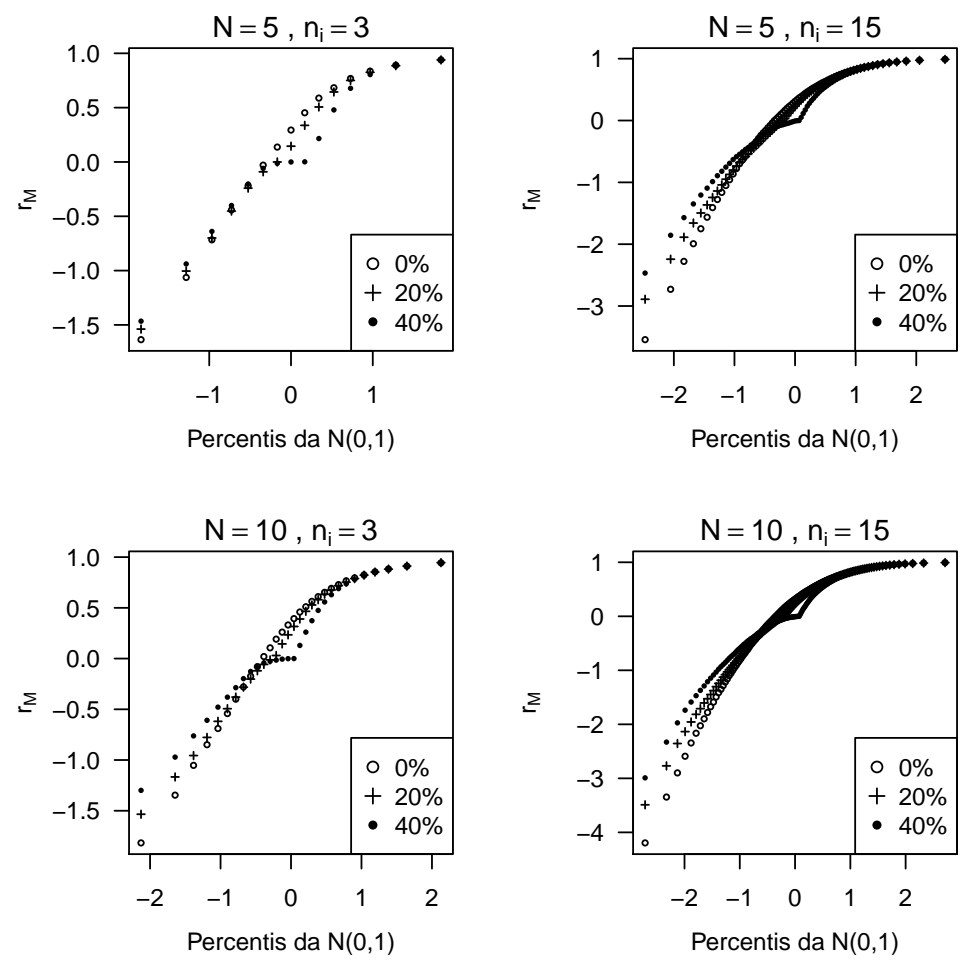

Figura 3.9: Gráfico normal de probabilidade para o resíduo $r_{M}$ no modelo Weibull normal com diferentes percentagens de censura para $N=5$ e $N=10$.

\subsubsection{Exemplo sobre transplante de medula óssea para pacientes com leucemia}

Avalos et al. (1993) apresentam um estudo sobre transplantes de medula óssea para pacientes com leucemia. A base de dados pode ser encontrada em Hanagal (2011) e refere-se à informação sobre pacientes com leucemia que foram tratados num dos 4 hospitais estudados. A variável resposta considerada na aplicação corresponde ao tempo de espera em dias para o transplante de medula. Adicionalmente, a base de dados contém variáveis tanto do paciente quanto do doador e a informação refere-se a 56 pacientes que foram submetidos ao processo de transplante com sucesso. As variáveis consideradas nessa aplicação são:

- $y$ : tempo de espera em dias para o transplante do paciente.

- $Z_{1}$ : idade do paciente em anos no inicio da espera.

- $Z_{2}$ : idade do doador em anos.

- $Z_{3}$ : sexo do paciente (0 mulher, 1 homem).

- $Z_{4}$ : sexo do doador (0 mulher, 1 homem).

- $Z_{5}$ : nível de citomegalovírus no paciente (0 negativo, 1 positivo).

- $Z_{6}$ : nível de citomegalovírus no doador (0 negativo, 1 positivo). 

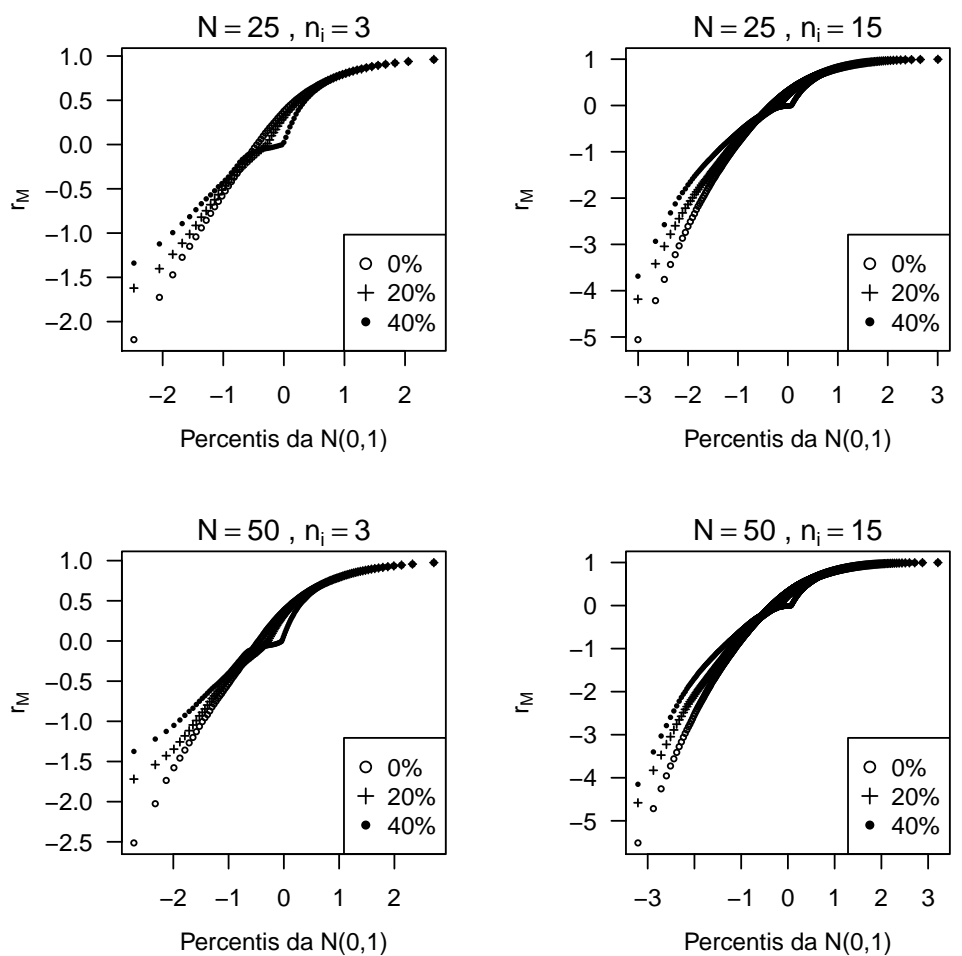

Figura 3.10: Gráfico normal de probabilidade para o resíduo $r_{M}$ no modelo Weibull normal com diferentes percentagens de censura para $N=25$ e $N=50$.

- $g$ : tipo de leucemia do paciente com três níveis, mielóide (0 baixa, 1 alta) ou leucemia linfoblástica aguda (2).

- $Z_{9}$ : hospital onde acontece o tratamento (1, 2, 3 e 4).

Na Figura 3.18 apresenta-se o gráfico de caixa da variável resposta tempo de espera em dias para o transplante em cada um dos hospitais. Observa-se que tanto a média do tempo quanto a variabilidade variam de hospital para hospital, por esta razão foi ajustado o modelo proposto nesse capítulo.

Foram, inicialmente, ajustados vários modelos usando diferentes subconjuntos de variáveis nos modelos dos parâmetros $\mu$ e $\sigma$ e o modelo que apresentou o maior valor da função log-verossimilhança foi o seguinte:

$$
\begin{aligned}
& y_{i j} \mid u_{1 i}, u_{2 i} \stackrel{i n d}{\sim} W E I 3\left(\mu_{i j}, \sigma_{i j}\right), \\
& \log \left(\mu_{i j}\right)=\beta_{10}+\beta_{11} Z_{1}+\beta_{12} Z_{2}+\beta_{13} Z_{3}+\beta_{14} Z_{4}+\beta_{15} g_{1}+\beta_{16} g_{2}+u_{1 i}, \\
& \log \left(\sigma_{i j}\right)=\beta_{20}+\beta_{21} Z_{1}+\beta_{22} Z_{2}+\beta_{23} Z_{3}+\beta_{24} Z_{4}+\beta_{26} Z_{6}+\beta_{27} g_{1}+\beta_{28} g_{2}+u_{2 i}, \\
& \operatorname{com} \quad i=1,2,3,4 \quad \text { e } \quad j=1,2, \ldots, n_{i}
\end{aligned}
$$

em que $i$ representa os hospitais e $j$ cada um dos pacientes. $u_{1 i}$ e $u_{2 i}$ correspondem aos interceptos aleatórios devidos aos hospitais com $u_{1 i} \sim N\left(0, \tau_{1}^{2}\right)$ e $u_{2 i} \sim N\left(0, \tau_{2}^{2}\right)$. Esse modelo ajustado apresentou como estimativas para a variabilidade dos efeitos aleatórios valores de 
Tabela 3.2: Média, desvio padrão, curtose e assimetria para $r_{o}$ no estudo de simulação do modelo Weibull normal com diferentes valores de $N, n_{i}$ e percentagem de censura.

\begin{tabular}{|c|c|c|c|c|c|c|}
\hline Censura & $N$ & $n_{i}$ & Média & D.P. & Curtose & Assimetria \\
\hline \multirow{8}{*}{$0 \%$} & \multirow{2}{*}{5} & 3 & $-0,05$ & 0,87 & 2,06 & 0,02 \\
\hline & & 15 & $-0,01$ & 0,94 & 2,94 & 0,31 \\
\hline & \multirow{2}{*}{10} & 3 & $-0,06$ & 0,80 & 2,21 & 0,28 \\
\hline & & 15 & $-0,01$ & 0,93 & 3,19 & 0,43 \\
\hline & \multirow{2}{*}{25} & 3 & $-0,06$ & 0,76 & 2,52 & 0,45 \\
\hline & & 15 & $-0,00$ & 0,93 & 3,69 & 0,59 \\
\hline & \multirow{2}{*}{50} & 3 & $-0,06$ & 0,75 & 2,71 & 0,52 \\
\hline & & 15 & $-0,01$ & 0,93 & 3,90 & 0,63 \\
\hline \multirow{8}{*}{$20 \%$} & \multirow{2}{*}{5} & 3 & $-0,38$ & 0,80 & 2,20 & $-0,26$ \\
\hline & & 15 & $-0,22$ & 0,83 & 2,91 & 0,26 \\
\hline & \multirow{2}{*}{10} & 3 & $-0,31$ & 0,76 & 2,38 & 0,06 \\
\hline & & 15 & $-0,24$ & 0,84 & 3,06 & 0,30 \\
\hline & \multirow{2}{*}{25} & 3 & $-0,30$ & 0,68 & 2,37 & 0,25 \\
\hline & & 15 & $-0,25$ & 0,86 & 3,17 & 0,27 \\
\hline & \multirow{2}{*}{50} & 3 & $-0,30$ & 0,64 & 2,35 & 0,36 \\
\hline & & 15 & $-0,26$ & 0,85 & 3,18 & 0,28 \\
\hline \multirow{8}{*}{$40 \%$} & \multirow{2}{*}{5} & 3 & $-0,88$ & 1,29 & 3,70 & $-1,12$ \\
\hline & & 15 & $-0,54$ & 0,86 & 2,86 & 0,20 \\
\hline & \multirow{2}{*}{10} & 3 & $-0,76$ & 1,02 & 3,02 & $-0,58$ \\
\hline & & 15 & $-0,59$ & 0,91 & 3,12 & 0,01 \\
\hline & \multirow{2}{*}{25} & 3 & $-0,66$ & 0,75 & 2,51 & $-0,02$ \\
\hline & & 15 & $-0,61$ & 0,93 & 3,17 & $-0,02$ \\
\hline & \multirow{2}{*}{50} & 3 & $-0,64$ & 0,68 & 2,43 & 0,13 \\
\hline & & 15 & $-0,61$ & 0,92 & 3,18 & 0,01 \\
\hline
\end{tabular}

D.P. corresponde ao desvio padrão.

$\hat{\tau}_{1}=1 e-10$ e $\hat{\tau}_{2}=2,04$. Por esta razão foi testada a hipótese $H_{0}:\left(\tau_{1}, \tau_{2}\right)^{\prime}=\mathbf{0}$ obtendose a estatística $\xi_{R V}=4,67$ indicando que $H_{0}$ é rejeitada. A seguir foi testada a hipótese $H_{0}: \tau_{1}=0$ obtendo-se uma estatística $\xi_{R V}=1,71 e-8$ não rejeitando $H_{0}$. Dos resultados conclui-se que é apropriado ajustar um modelo só com intercepto aleatório no parâmetro $\sigma$ da distribuição. Os resultados para este modelo são apresentados na Tabela 3.7. Da tabela observa-se que as variáveis idade do paciente e sexo do doador são significativas, além disso, no parâmetro $\mu$ elas têm um efeito positivo, enquanto no parâmetro o efeito é negativo. Observa-se também que à medida que o tipo de leucemia cresce o efeito no preditor linear de $\mu$ é negativo, enquanto o efeito no preditor linear de $\sigma$ é positivo.

Na Figura 3.19 apresentam-se gráficos de normalidade para os resíduos com os respectivos valores-p do teste de normalidade de Anderson-Darling. Observa-se que só os resíduos $r_{d}$ e $r_{q}$ seguem uma distribuição normal. 
Tabela 3.3: Média, desvio padrão, curtose e assimetria para $r_{q}$ no estudo de simulação do modelo Weibull normal com diferentes valores de $N, n_{i}$ e percentagem de censura.

\begin{tabular}{|c|c|c|c|c|c|c|}
\hline Censura & $N$ & $n_{i}$ & Média & D.P. & Curtose & Assimetria \\
\hline \multirow{8}{*}{$0 \%$} & \multirow{2}{*}{5} & 3 & $-0,04$ & 0,91 & 1,95 & $-0,06$ \\
\hline & & 15 & $-0,00$ & 0,97 & 2,64 & $-0,00$ \\
\hline & \multirow{2}{*}{10} & 3 & $-0,06$ & 0,84 & 2,09 & 0,06 \\
\hline & & 15 & $-0,00$ & 0,97 & 2,78 & 0,01 \\
\hline & \multirow{2}{*}{25} & 3 & $-0,06$ & 0,80 & 2,29 & 0,08 \\
\hline & & 15 & 0,01 & 0,96 & 2,86 & 0,03 \\
\hline & \multirow{2}{*}{50} & 3 & $-0,06$ & 0,79 & 2,40 & 0,05 \\
\hline & & 15 & 0,00 & 0,95 & 2,87 & 0,02 \\
\hline \multirow{8}{*}{$20 \%$} & \multirow{2}{*}{5} & 3 & $-0,24$ & 0,91 & 1,99 & $-0,10$ \\
\hline & & 15 & $-0,19$ & 0,89 & 2,70 & 0,05 \\
\hline & \multirow{2}{*}{10} & 3 & $-0,29$ & 0,81 & 2,19 & $-0,03$ \\
\hline & & 15 & $-0,21$ & 0,91 & 2,79 & 0,04 \\
\hline & \multirow{2}{*}{25} & 3 & $-0,29$ & 0,74 & 2,27 & 0,01 \\
\hline & & 15 & $-0,23$ & 0,92 & 2,82 & 0,01 \\
\hline & \multirow{2}{*}{50} & 3 & $-0,28$ & 0,71 & 2,30 & 0,04 \\
\hline & & 15 & $-0,24$ & 0,92 & 2,81 & 0,00 \\
\hline \multirow{8}{*}{$40 \%$} & \multirow{2}{*}{5} & 3 & $-0,65$ & 1,23 & 2,60 & $-0,52$ \\
\hline & & 15 & $-0,52$ & 0,92 & 2,55 & 0,12 \\
\hline & \multirow{2}{*}{10} & 3 & $-0,72$ & 1,01 & 2,42 & $-0,28$ \\
\hline & & 15 & $-0,56$ & 0,97 & 2,67 & $-0,00$ \\
\hline & \multirow{2}{*}{25} & 3 & $-0,65$ & 0,81 & 2,21 & 0,00 \\
\hline & & 15 & $-0,58$ & 0,97 & 2,68 & $-0,02$ \\
\hline & \multirow{2}{*}{50} & 3 & $-0,63$ & 0,75 & 2,14 & 0,10 \\
\hline & & 15 & $-0,58$ & 0,96 & 2,68 & 0,01 \\
\hline
\end{tabular}

D.P. corresponde ao desvio padrão.

\subsection{Conclusões}

Neste capítulo apresentou-se o modelo de dois níveis com resposta Weibull e interceptos aleatórios normais nos modelos para os parâmetros $\mu$ e $\sigma$ da distribuição. As estimativas dos parâmetros do modelo foram obtidos por meio da maximização da função de verossimilhança e para tanto foi necessário o desenvolvimento de rotinas computacionais específicas.

Três tipos de resíduos foram propostos para verificar a adequação desse novo modelo, sendo eles o resíduo ordinário, o resíduo componente do desvio e o resíduo quantil normal aleatorizado.

Por se tratar de um modelo misto, considerou-se o teste de componente de variância para estudar a hipótese da existência de efeitos aleatórios nos modelos dos parâmetros da distribuição da variável resposta. Em particular, foi apresentado o teste de razão de verossimilhanças para estudar três situações: efeitos aleatórios nos modelos dos dois parâmetros, efeito aleatório no modelo do parâmetro $\mu$ ou no modelo do parâmetro $\sigma$.

Adicionalmente, desenvolveu-se um estudo de simulação Monte Carlo para examinar a distribuição empírica das componentes do vetor $\hat{\boldsymbol{\theta}}$, a distribuição empírica dos resíduos pro- 
Tabela 3.4: Média, desvio padrão, curtose e assimetria para $r_{d}$ no estudo de simulação do modelo Weibull normal com diferentes valores de $N, n_{i}$ e percentagem de censura.

\begin{tabular}{|c|c|c|c|c|c|c|}
\hline Censura & $N$ & $n_{i}$ & Média & D.P. & Curtose & Assimetria \\
\hline \multirow{8}{*}{$0 \%$} & \multirow{2}{*}{5} & 3 & 0,16 & 0,79 & 1,93 & $-0,04$ \\
\hline & & 15 & 0,34 & 0,99 & 2,62 & $-0,02$ \\
\hline & \multirow{2}{*}{10} & 3 & 0,40 & 0,86 & 2,09 & $-0,07$ \\
\hline & & 15 & 0,34 & 0,98 & 2,76 & $-0,03$ \\
\hline & \multirow{2}{*}{25} & 3 & 0,41 & 0,82 & 2,28 & $-0,09$ \\
\hline & & 15 & 0,34 & 0,98 & 2,84 & $-0,05$ \\
\hline & \multirow{2}{*}{50} & 3 & 0,41 & 0,80 & 2,39 & $-0,06$ \\
\hline & & 15 & 0,34 & 0,97 & 2,85 & $-0,04$ \\
\hline \multirow{8}{*}{$20 \%$} & \multirow{2}{*}{5} & 3 & $-0,10$ & 0,81 & 2,07 & $-0,13$ \\
\hline & & 15 & 0,18 & 1,05 & 2,19 & $-0,07$ \\
\hline & \multirow{2}{*}{10} & 3 & 0,17 & 0,88 & 2,10 & $-0,22$ \\
\hline & & 15 & 0,23 & 1,06 & 2,44 & $-0,02$ \\
\hline & \multirow{2}{*}{25} & 3 & 0,29 & 0,92 & 2,23 & $-0,16$ \\
\hline & & 15 & 0,22 & 1,05 & 2,52 & $-0,01$ \\
\hline & \multirow{2}{*}{50} & 3 & 0,29 & 0,92 & 2,28 & $-0,16$ \\
\hline & & 15 & 0,23 & 1,04 & 2,56 & $-0,01$ \\
\hline \multirow{8}{*}{$40 \%$} & \multirow{2}{*}{5} & 3 & $-0,54$ & 0,56 & 2,02 & $-0,58$ \\
\hline & & 15 & $-0,16$ & 0,84 & 2,14 & 0,05 \\
\hline & \multirow{2}{*}{10} & 3 & $-0,45$ & 0,50 & 2,43 & $-0,58$ \\
\hline & & 15 & 0,12 & 1,04 & 2,42 & 0,29 \\
\hline & \multirow{2}{*}{25} & 3 & 0,17 & 0,90 & 2,00 & 0,09 \\
\hline & & 15 & 0,13 & 1,03 & 2,51 & 0,29 \\
\hline & \multirow{2}{*}{50} & 3 & 0,20 & 0,92 & 2,08 & 0,14 \\
\hline & & 15 & 0,13 & 1,03 & 2,53 & 0,29 \\
\hline
\end{tabular}

D.P. corresponde ao desvio padrão.

postos, assim como a distribuição empírica da estatística e o comportamento do teste da razão de verossimilhanças. Em relação à distribuição das componentes de $\hat{\boldsymbol{\theta}}$ foi encontrado que, à medida que o número de observações aumenta, pelo aumento de $N$ ou $n_{i}$, a distribuição das componentes fica mais concentrada ao redor do verdadeiro valor da componente. Em relação à distribuição empírica dos resíduos, encontrou-se que a distribuição do resíduo ordinário não é normal enquanto as distribuições empíricas dos resíduos componente do desvio e quantil normal aleatorizado ficaram próximas da normal à medida em que o número de observações disponíveis aumenta. Portanto, recomenda-se o uso dos residuos para verificar a qualidade do ajuste do modelo. Com respeito ao teste da razão de verossimilhanças, encontrou-se que a distribuição empírica da estatística, sob a hipótese nula verdadeira, pode ser considerada como uma combinação $0,5: 0,5$ de $\chi_{0}^{2}$ e $\chi_{1}^{2}$.

Uma vantagem do uso desse modelo proposto é a possibilidade de analisar fenómenos nos quais o domínio da variável resposta corresponde aos reais positivos e, caso a variável esteja relacionada ao tempo, a inclusão de observações censuradas à direita. Na modelagem dos parâmetros da distribuição da variável resposta é possível usar tanto efeitos fixos quanto 


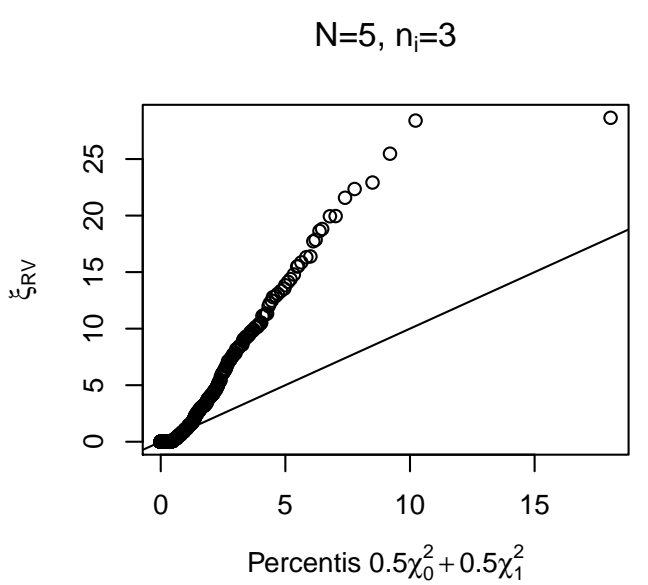

$\mathrm{N}=5, \mathrm{n}_{\mathrm{i}}=10$

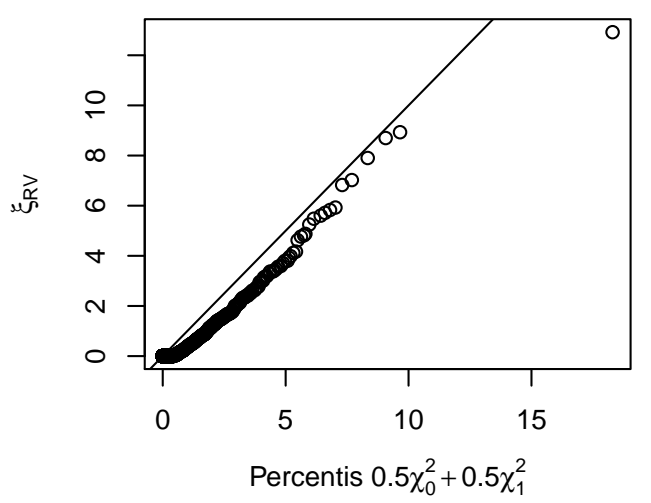

$\mathrm{N}=5, \mathrm{n}_{\mathrm{i}}=5$



$\mathrm{N}=5, \mathrm{n}_{\mathrm{i}}=15$

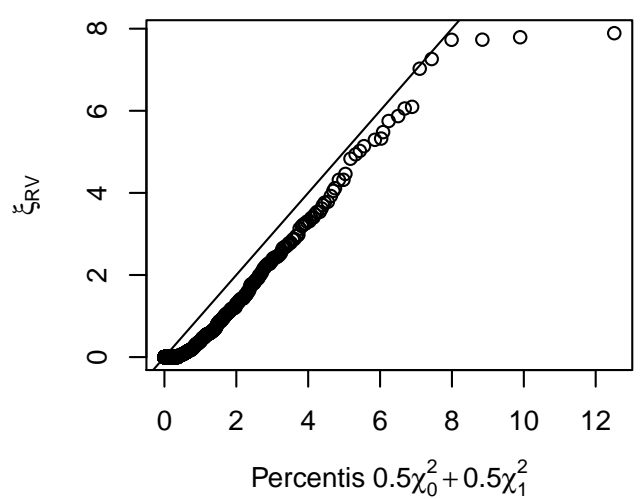

Figura 3.11: Gráfico de probabilidade das estatísticas $\xi_{R V}$ versus os percentis da $0.5 \chi_{0}^{2}+0.5 \chi_{1}^{2}$ para as combinaçôes de $N=5$ e $n_{i}=3,5,10,15$ com $\tau_{1}=\tau_{2}=0$.

efeitos aleatórios normais e a estimação dos parâmetros do modelo é realizada de forma conjunta pelo método de máxima verossimilhança sem a necessidade de especificação de distribuições a priori ou hiperparâmetros. 
$\mathrm{N}=10, \mathrm{n}_{\mathrm{i}}=3$

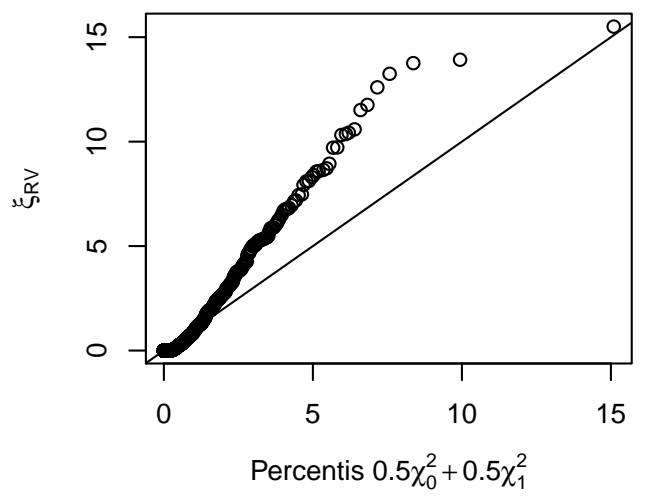

$\mathrm{N}=10, \mathrm{n}_{\mathrm{i}}=10$

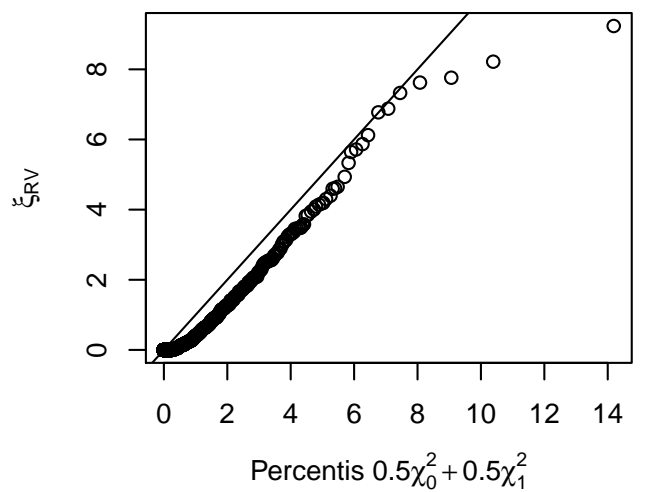

$\mathrm{N}=10, \mathrm{n}_{\mathrm{i}}=5$

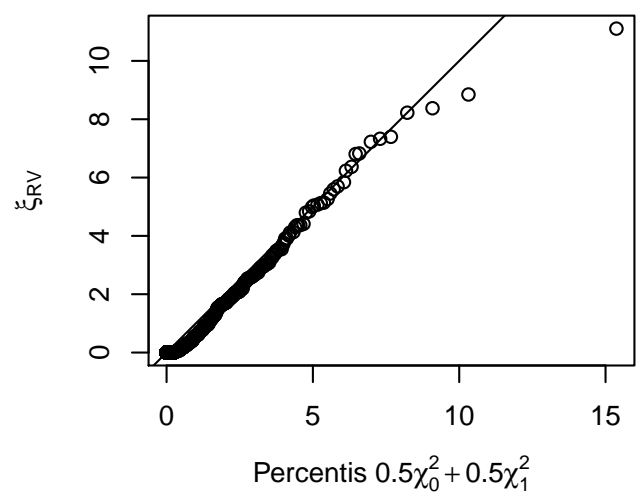

$\mathrm{N}=10, \mathrm{n}_{\mathrm{i}}=15$

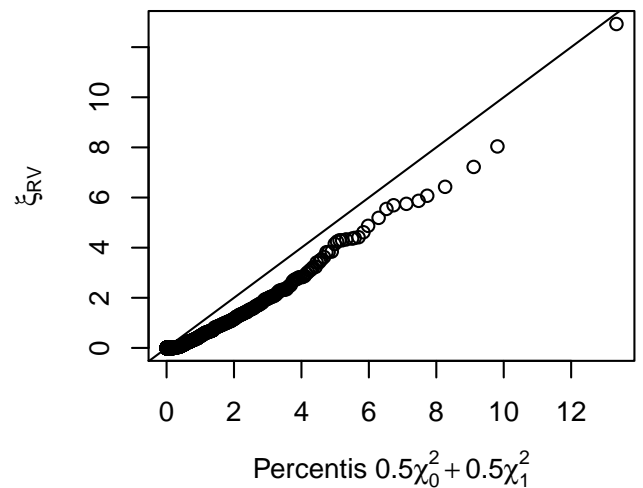

Figura 3.12: Gráfico de probabilidade das estatísticas $\xi_{R V}$ versus os percentis da $0.5 \chi_{0}^{2}+0.5 \chi_{1}^{2}$ para as combinaçôes de $N=10$ e $n_{i}=3,5,10,15$ com $\tau_{1}=\tau_{2}=0$. 
$\mathrm{N}=25, \mathrm{n}_{\mathrm{i}}=3$

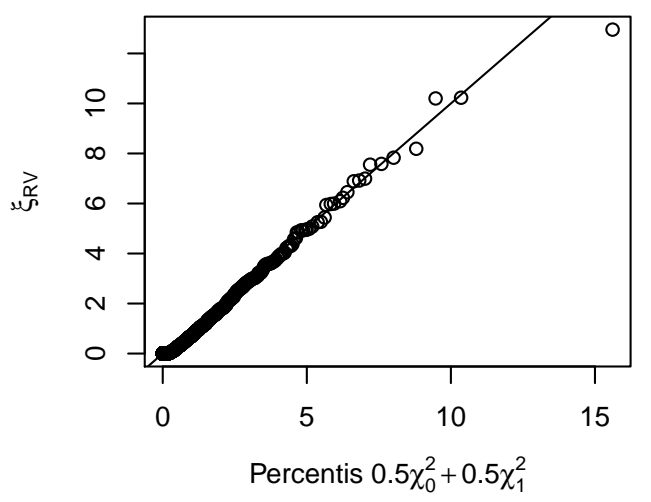

$\mathrm{N}=25, \mathrm{n}_{\mathrm{i}}=10$

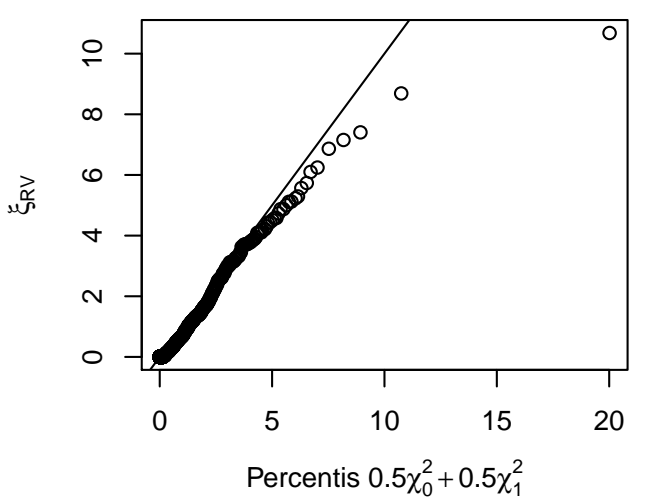

$\mathrm{N}=25, \mathrm{n}_{\mathrm{i}}=5$

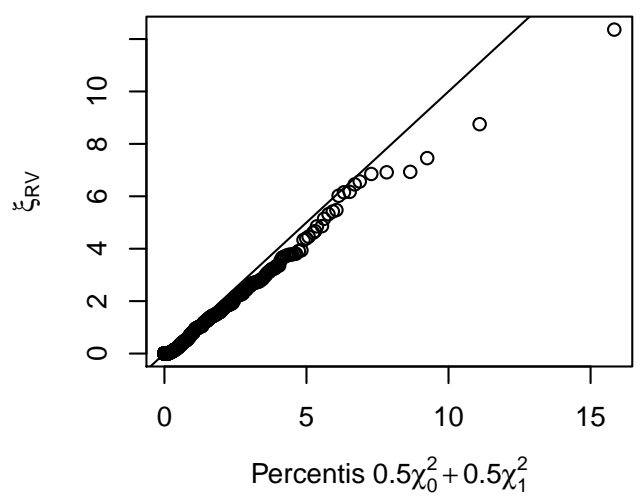

$\mathrm{N}=25, \mathrm{n}_{\mathrm{i}}=15$

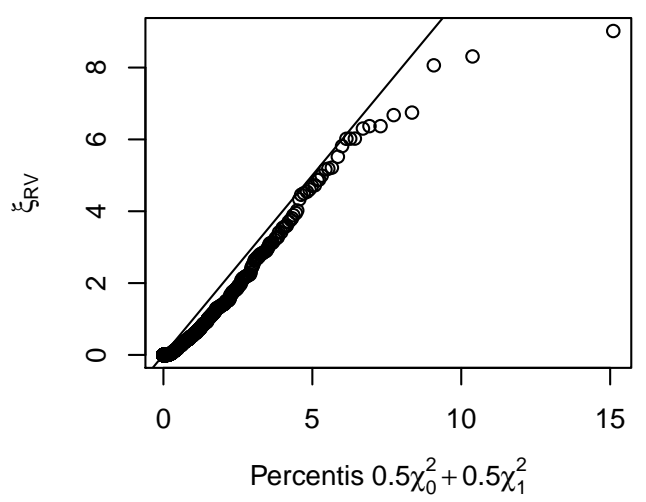

Figura 3.13: Gráfico de probabilidade das estatísticas $\xi_{R V}$ versus os percentis da $0.5 \chi_{0}^{2}+0.5 \chi_{1}^{2}$ para as combinaçôes de $N=25$ e $n_{i}=3,5,10,15$ com $\tau_{1}=\tau_{2}=0$. 
$\mathrm{N}=50, \mathrm{n}_{\mathrm{i}}=3$

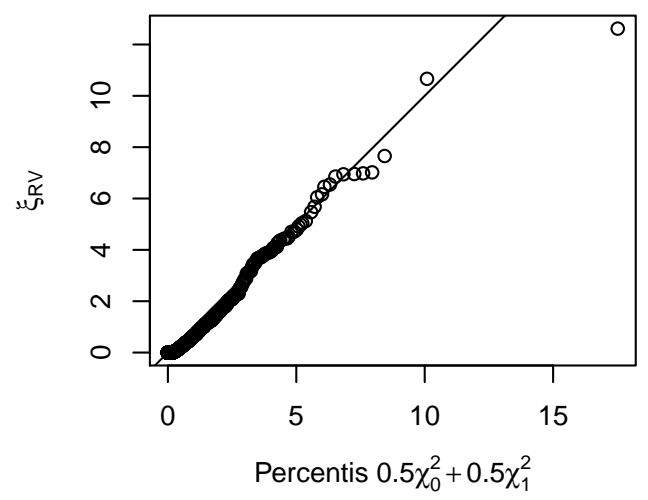

$\mathrm{N}=50, \mathrm{n}_{\mathrm{i}}=10$

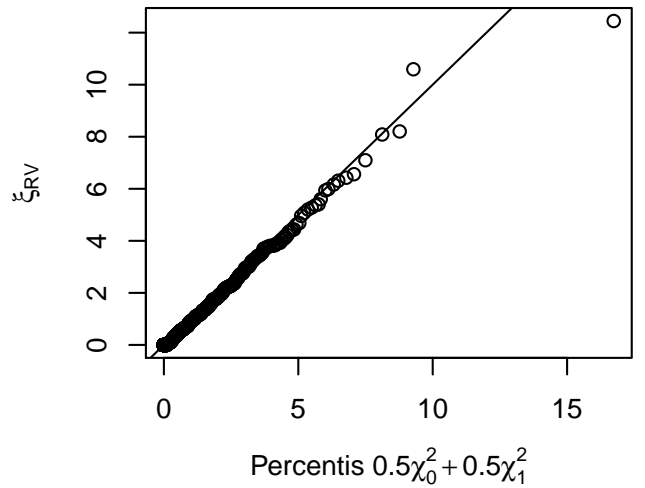

$\mathrm{N}=50, \mathrm{n}_{\mathrm{i}}=5$

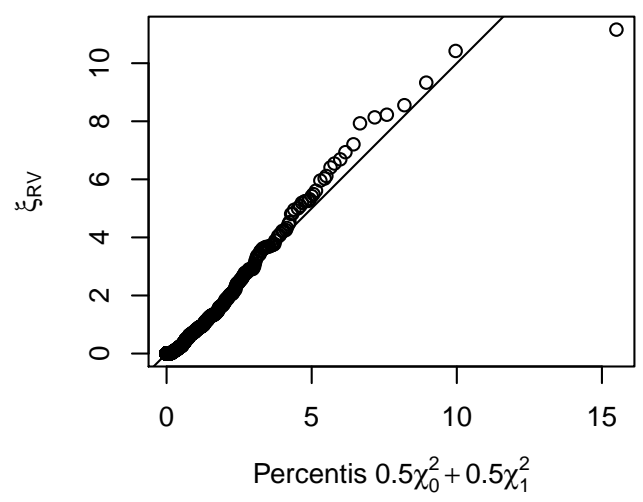

$\mathrm{N}=50, \mathrm{n}_{\mathrm{i}}=15$

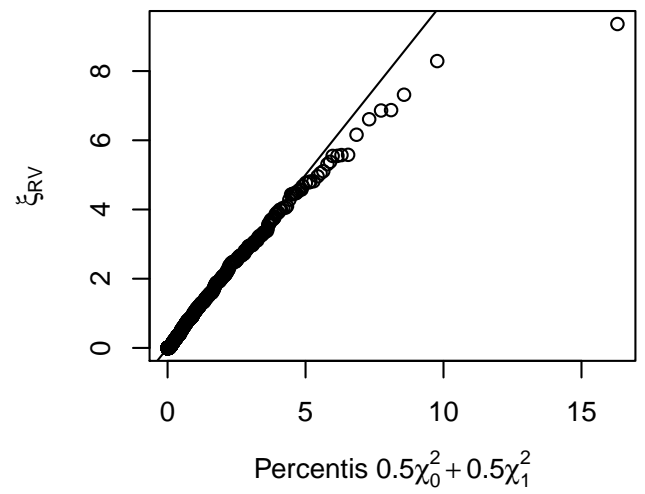

Figura 3.14: Gráfico de probabilidade das estatísticas $\xi_{R V}$ versus os percentis da $0.5 \chi_{0}^{2}+0.5 \chi_{1}^{2}$ para as combinaçôes de $N=50$ e $n_{i}=3,5,10,15$ com $\tau_{1}=\tau_{2}=0$. 
Tabela 3.5: Percetagem de rejeiçôes de $H_{0}:\left(\tau_{1}, \tau_{2}\right)=\mathbf{0}$ no teste $R V$ a um nível de significância de $5 \%$.

\begin{tabular}{rccrrrr} 
& & & \multicolumn{4}{c}{$n_{i}$} \\
\cline { 3 - 7 }$N$ & $\tau_{1}$ & $\tau_{2}$ & 3 & 5 & 10 & 15 \\
\hline & 0 & 0 & 16,0 & 6,8 & 4,1 & 4,8 \\
5 & 0 & 0,5 & 33,9 & 38,6 & 62,1 & 74,5 \\
5 & 0,5 & 0 & 66,7 & 78,1 & 94,6 & 98,0 \\
& 0,5 & 0,5 & 66,3 & 81,7 & 95,9 & 99,4 \\
& 1 & 1 & 92,2 & 98,6 & 99,9 & 99,9 \\
\hline & 0 & 0 & 11,8 & 5,8 & 4,8 & 4,3 \\
& 0 & 0,5 & 44,8 & 65,1 & 91,7 & 97,3 \\
10 & 0,5 & 0 & 87,5 & 97,7 & 99,9 & 100,0 \\
& 0,5 & 0,5 & 86,6 & 98,6 & 99,9 & 100,0 \\
& 1 & 1 & 99,6 & 100,0 & 100,0 & 100,0 \\
\hline & 0 & 0 & 7,4 & 6,1 & 6,3 & 5,8 \\
& 0 & 0,5 & 58,3 & 81,1 & 98,0 & 99,4 \\
15 & 0,5 & 0 & 95,2 & 99,9 & 100,0 & 100,0 \\
& 0,5 & 0,5 & 96,7 & 99,9 & 100,0 & 100,0 \\
& 1 & 1 & 99,9 & 100,0 & 100,0 & 100,0 \\
\hline & 0 & 0 & 7,6 & 5,9 & 6,9 & 5,8 \\
& 0 & 0,5 & 78,7 & 95,8 & 100,0 & 100,0 \\
25 & 0,5 & 0 & 99,3 & 100,0 & 100,0 & 100,0 \\
& 0,5 & 0,5 & 99,7 & 100,0 & 100,0 & 100,0 \\
& 1 & 1 & 100,0 & 100,0 & 100,0 & 100,0 \\
\hline & 0 & 0 & 6,5 & 7,4 & 7,0 & 8,0 \\
& 0 & 0,5 & 97,5 & 100,0 & 100,0 & 100,0 \\
50 & 0,5 & 0 & 100,0 & 100,0 & 100,0 & 100,0 \\
& 0,5 & 0,5 & 100,0 & 100,0 & 100,0 & 100,0 \\
& 1 & 1 & 100,0 & 100,0 & 100,0 & 100,0 \\
\hline & & & & & &
\end{tabular}

Tabela 3.6: Estimativas (e erros padrão em parênteses) para os parâmetros do modelo da base de dados simulada.

\begin{tabular}{|cc|c|c|}
\hline Parâmetro & Valor real & Weibull-normal & GAMLSS \\
\hline$\beta_{11}$ & $-1,0$ & $-1,171(0,540)$ & $-1,325(0,334)$ \\
$\beta_{12}$ & 0,5 & $0,334(0,261)$ & $0,393(0,162)$ \\
$\beta_{21}$ & 1,3 & $1,235(0,753)$ & $1,668(0,348)$ \\
$\beta_{22}$ & 0,3 & $0,540(0,664)$ & $0,760(0,358)$ \\
$\tau_{1}$ & 0,8 & $0,464(0,046)$ & $0,555($ n.d.) \\
$\tau_{2}$ & 1,0 & $0,705(0,272)$ & 0,662 (n.d.) \\
\hline$l(\boldsymbol{\theta})$ & $-19,565$ & $-14,760$ & $-16,748$ \\
\hline
\end{tabular}

n.d. significa não disponível. 


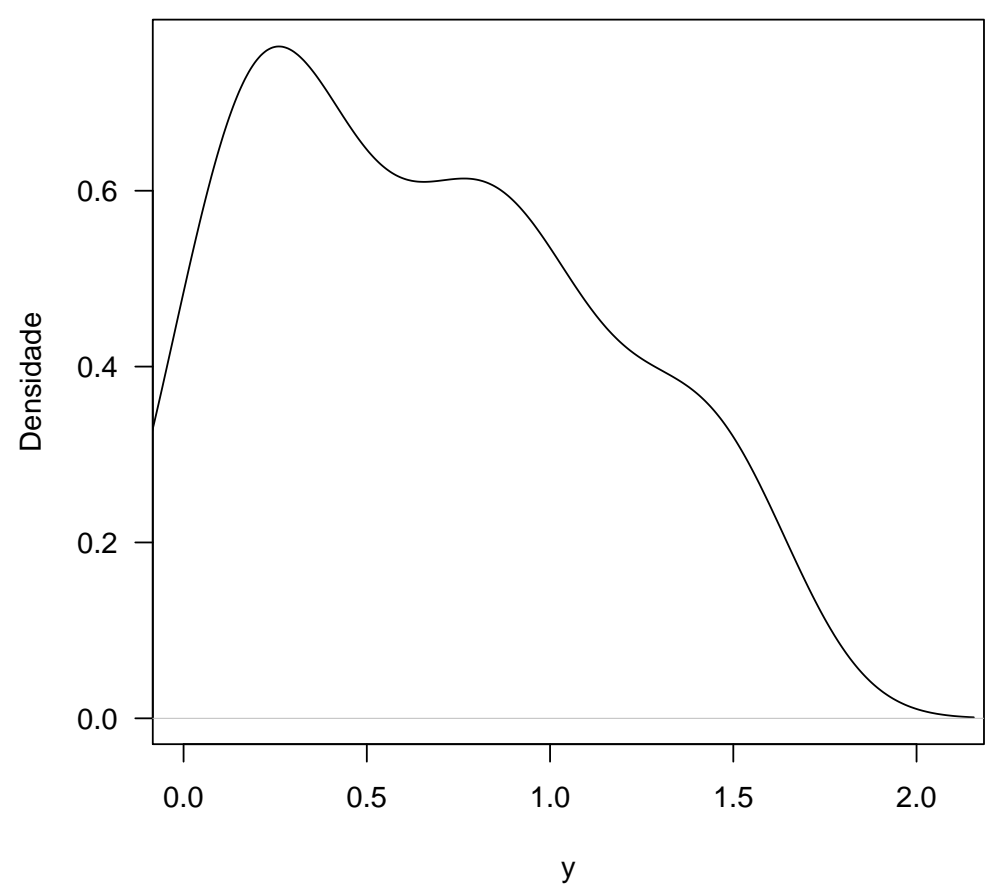

Figura 3.15: Estimativa da função densidade para a variável resposta y da base de dados simulada com resposta Weibull e efeitos aleatórios normal.

$\mathrm{u}_{1}$

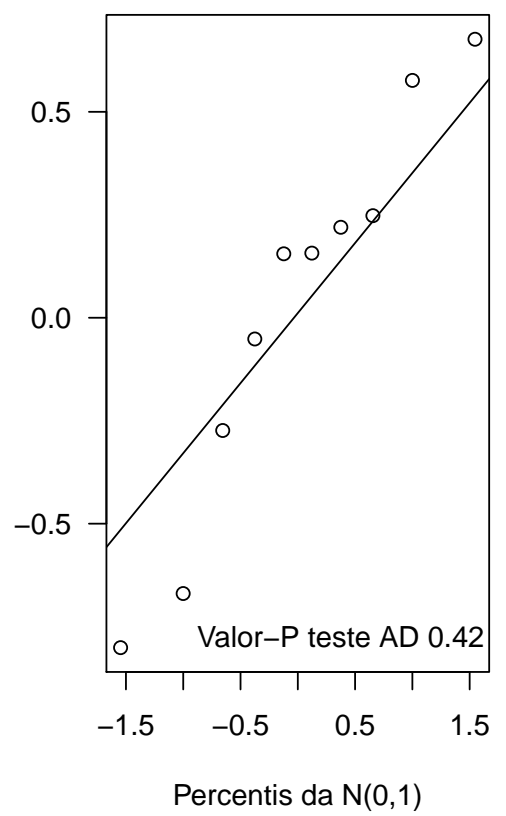

$\mathrm{u}_{2}$

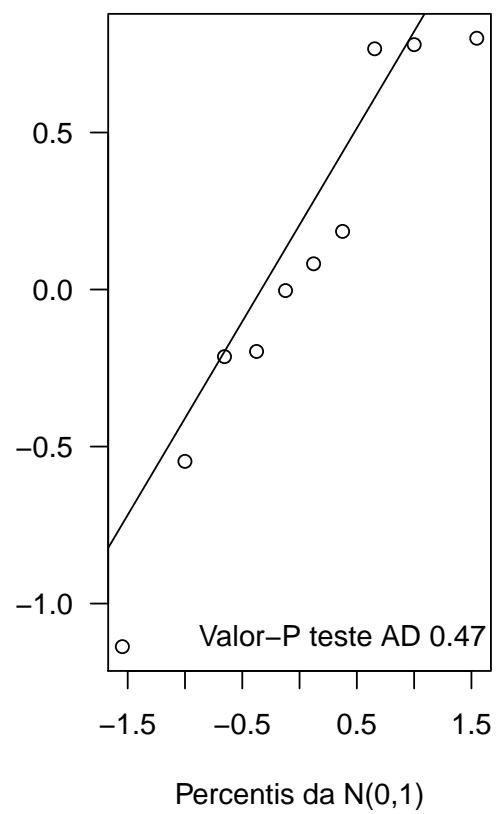

Figura 3.16: Gráficos normais de probabilidade dos efeitos aleatórios preditos $u_{1}$ e $u_{2}$ para a base de dados simulada com resposta Weibull e efeitos aleatórios normal. 
$r_{0}$

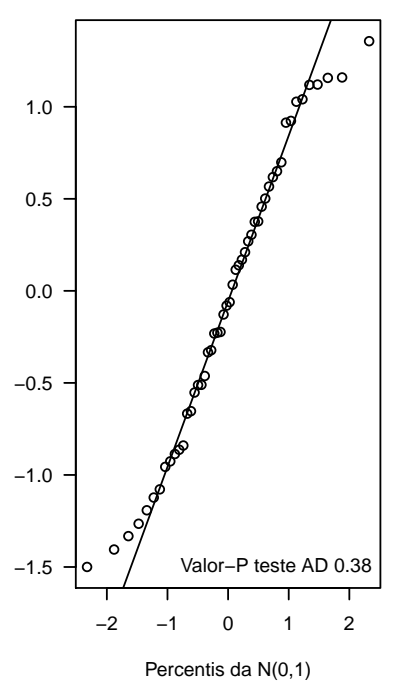

$r_{d}$

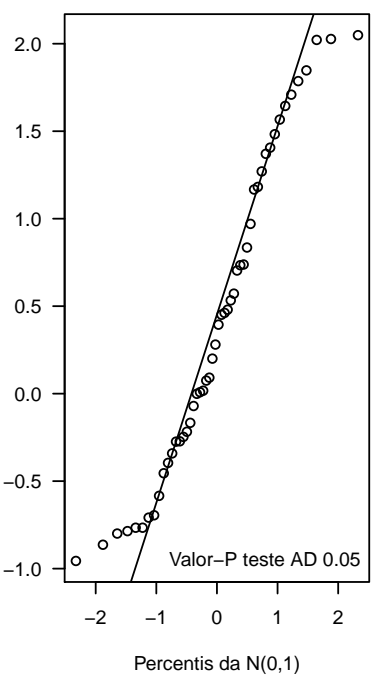

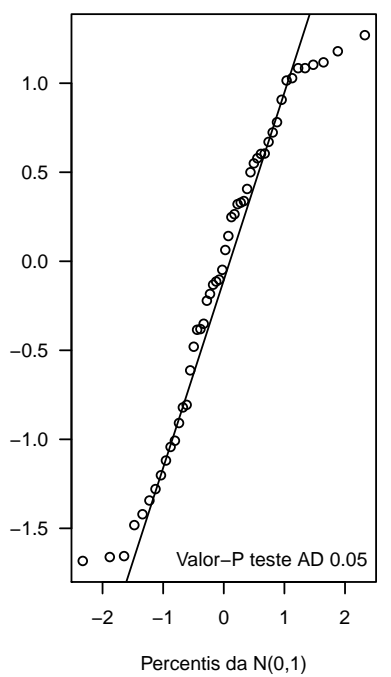

Figura 3.17: Gráficos normais de probabilidade dos resíduos $r_{o}, r_{d}$ e $r_{q}$ para base de dados simulada com resposta Weibull e efeitos aleatórios normal.

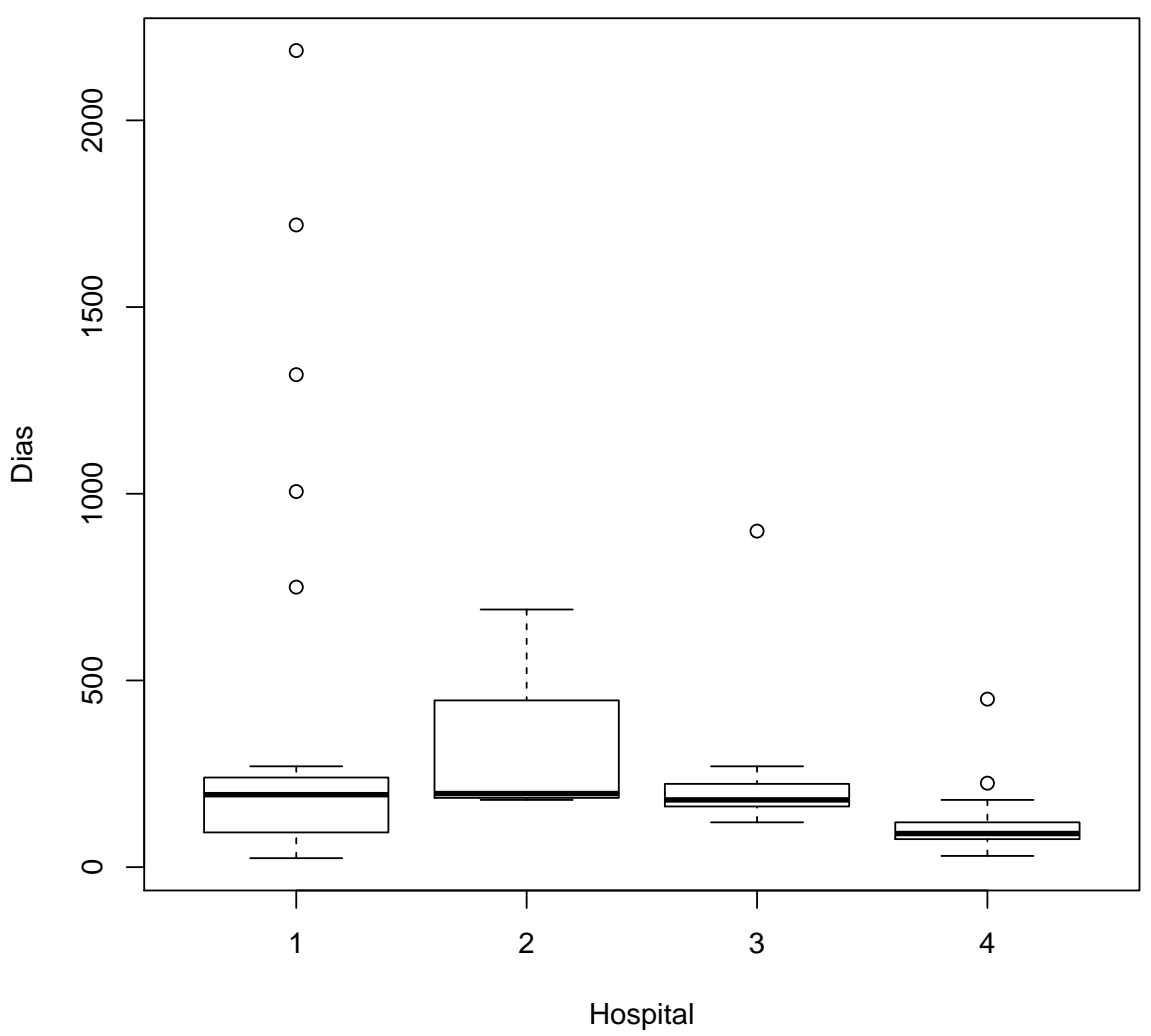

Figura 3.18: Gráfico de caixa para tempo até o transplante dados os hospitais. 
Tabela 3.7: Estimativas e erros padrão dos parâmetros referentes ao modelo para a base de dados leucemia.

\begin{tabular}{lcrr}
\hline Efeito & Parâmetro & Estimativa & E. padrão \\
\hline Constante & $\beta_{10}$ & $-9,81$ & 1,49 \\
Idade paciente & $\beta_{11}$ & 0,18 & 0,07 \\
Idade doador & $\beta_{12}$ & $-0,06$ & 0,07 \\
Paciente-homem & $\beta_{13}$ & $-1,58$ & 1,11 \\
Doador-homem & $\beta_{14}$ & 3,04 & 1,65 \\
Tipo leucemia 1 & $\beta_{15}$ & $-6,32$ & 1,91 \\
Tipo leucemia 2 & $\beta_{16}$ & $-3,30$ & 2,29 \\
\hline Constante & $\beta_{20}$ & 3,18 & 0,26 \\
Idade paciente & $\beta_{21}$ & $-0,02$ & 0,01 \\
Idade doador & $\beta_{22}$ & 0,01 & 0,01 \\
Paciente-homem & $\beta_{23}$ & 0,19 & 0,12 \\
Doador-homem & $\beta_{24}$ & $-0,30$ & 0,16 \\
Citomegalovirus doador & $\beta_{26}$ & $-0,03$ & 0,03 \\
Tipo leucemia 1 & $\beta_{27}$ & 0,95 & 0,21 \\
Tipo leucemia 2 & $\beta_{28}$ & 0,46 & 0,28 \\
\hline Variabilidade de $u_{2 i}$ & $\tau_{2}$ & 2,04 & 0,34 \\
\hline$l(\boldsymbol{\theta})$ & $-356,20$ & & \\
\hline
\end{tabular}

$r_{0}$

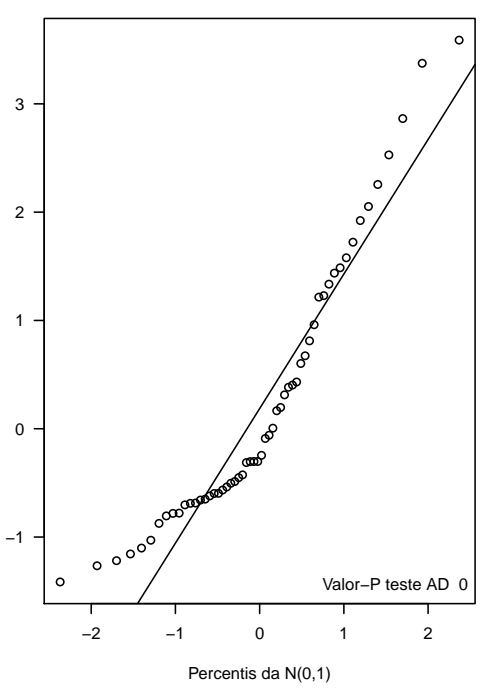

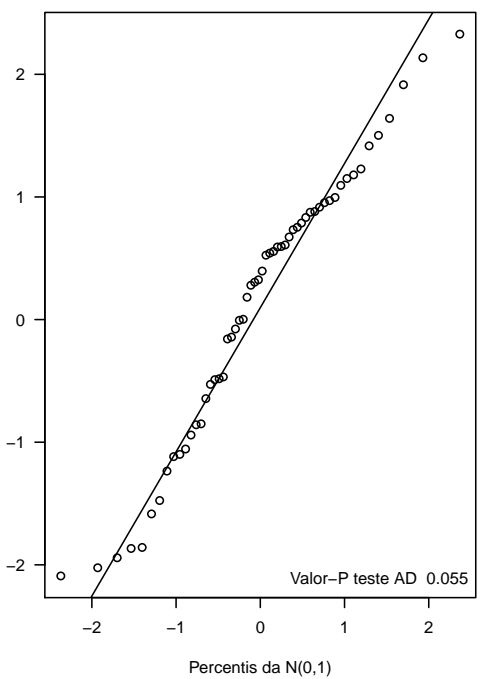



Figura 3.19: Gráficos normais de probabilidade dos resíduos $r_{o}, r_{d}$ e $r_{q}$ para a base de dados leucemia. 


\section{Capítulo 4}

\section{Modelo multinível Weibull com dois níveis e interceptos aleatórios log-gama}

O modelo multinível de dois níveis com variável resposta Weibull no qual são considerados interceptos aleatórios com distribuição log-gama nos modelos dos dois parâmetros da distribuição da variável resposta é descrito neste capítulo. Efeitos aleatórios com distribuição log-gama têm sido usados em modelos mistos Poisson por Fabio et al. (2012), em modelos lineares mistos por Zhang et al. (2008), em modelos lineares generalizados hierárquicos para respostas binomial, Poisson e gama por Lee et al. (2006) e em modelos de sobrevivência com resposta Weibull por Morris e Christiansen (1995) e Sahu et al. (1997). A inferência nesse novo modelo é realizada sob a abordagem marginal. Nas primeiras duas seções apresenta-se a estrutura do modelo, assim como o método para estimação dos parâmetros. Na seção 4.1 é exposta uma proposta para predizer os efeitos aleatórios e são apresentados três tipos de resíduos diferentes. No final do capítulo, uma aplicação do modelo é apresentada ilustrando o modelo proposto.

\subsection{Apresentação do modelo}

A estrutura hierárquica de dois níveis considerada para este modelo com resposta Weibull é também representada pela Figura 3.1. Supondo $y_{i j}$ como a $j$-ésima medida do $i$-ésimo grupo, a estrutura do modelo é a seguinte:

$$
\begin{aligned}
y_{i j} \mid u_{1 i}, u_{2 i} & \stackrel{i n d}{\sim} \operatorname{WEI}\left(\mu_{i j}, \sigma_{i j}\right), \\
u_{1 i} & \stackrel{i i d}{\sim} L G\left(\tau_{1}\right) \\
\mathrm{e} \quad u_{2 i} & \stackrel{i i d}{\sim} L G\left(\tau_{2}\right), \quad i=1,2, \ldots, N, \quad j=1,2, \ldots, n_{i}
\end{aligned}
$$

em que $L G(\tau)$ representa a distribuição log-gama com função de densidade dada por

$$
f(u \mid \tau)=\frac{(1 / \tau)^{1 / \tau}}{\Gamma(1 / \tau)} e^{\left(u-e^{u}\right) / \tau},
$$


a qual é indexada pero parâmetro $\tau$ para evitar problemas de identificabilidade. Os parâmetros $\mu$ e $\sigma$ são modelados por

$$
\begin{aligned}
& \log \left(\mu_{i j}\right)=\boldsymbol{X}_{1 i, j .}^{\top} \cdot \boldsymbol{\beta}_{1}+u_{1 i}, \\
& \log \left(\sigma_{i j}\right)=\boldsymbol{X}_{2 i, j}^{\top} \cdot \boldsymbol{\beta}_{2}+u_{2 i},
\end{aligned}
$$

em que $u_{1}$ e $u_{2}$ são independentes. As matrizes $\boldsymbol{X}_{1 i}$ e $\boldsymbol{X}_{2 i}$ são matrizes de planejamento conhecidas para o grupo $i, \boldsymbol{X}_{1 i, j}$. e $\boldsymbol{X}_{2 i, j}$. correspondem à $j$-ésima linha de $\boldsymbol{X}_{1 i}$ e $\boldsymbol{X}_{2 i}$ respectivamente; $\boldsymbol{\beta}_{1}$ e $\boldsymbol{\beta}_{2}$ são vetores de parâmetros desconhecidos. Isolando $\mu_{i j}$ e $\sigma_{i j}$ das expressões (4.3 e 4.4) tem-se que $\mu_{i j}=\exp \left(u_{1 i}\right) \exp \left(\boldsymbol{X}_{1 i, j} \cdot \boldsymbol{\beta}_{1}\right)$ e $\sigma_{i j}=\exp \left(u_{2 i}\right) \exp \left(\boldsymbol{X}_{2 i, j} \cdot \boldsymbol{\beta}_{2}\right)$, em que $\exp \left(u_{i 1}\right)$ e $\exp \left(u_{i 2}\right)$ têm distribuição gama com média 1 e variâncias $\tau_{1}$ e $\tau_{2}$, respectivamente. Por essa razão afirma-se que os efeitos aleatórios no modelo têm estrutura multiplicativa. Gráficos da função de densidade para vários valores do parâmetro $\tau$ são apresentados na Seção 5.1.

Em forma vetorial, os parâmetros $\mu$ e $\sigma$ para cada grupo têm a seguinte estrutura:

$$
\begin{aligned}
\log \left(\boldsymbol{\mu}_{i}\right) & =\boldsymbol{X}_{1 i} \boldsymbol{\beta}_{1}+\mathbf{1}_{n_{i}} u_{1 i} \\
\text { e } \quad \log \left(\boldsymbol{\sigma}_{i}\right) & =\boldsymbol{X}_{2 i} \boldsymbol{\beta}_{2}+\mathbf{1}_{n_{i}} u_{2 i},
\end{aligned}
$$

em que

$$
\begin{aligned}
\boldsymbol{\mu}_{i} & =\left(\mu_{i 1}, \mu_{i 2}, \ldots, \mu_{i n_{i}}\right)^{\top}, \\
\log \left(\boldsymbol{\mu}_{i}\right) & =\left(\log \left(\mu_{i 1}\right), \log \left(\mu_{i 2}\right), \ldots, \log \left(\mu_{i n_{i}}\right)\right)^{\top}, \\
\boldsymbol{\sigma}_{i} & =\left(\sigma_{i 1}, \sigma_{i 2}, \ldots, \sigma_{i n_{i}}\right)^{\top} \\
\text { e } \quad \log \left(\boldsymbol{\sigma}_{i}\right) & =\left(\log \left(\sigma_{i 1}\right), \log \left(\sigma_{i 2}\right), \ldots, \log \left(\sigma_{i n_{i}}\right)\right)^{\top},
\end{aligned}
$$

com $\mathbf{1}_{n_{i}}$ indicando um vetor coluna de $1^{\prime} s$ de dimensão $n_{i}$.

\subsection{Estimação dos parâmetros do modelo}

As estimativas dos parâmetros do modelo são obtidos maximizando o logaritmo da função de verossimilhança, a qual é construida usando o enfoque marginal, partindo da densidade condicional do vetor de observações para para $i$-ésimo grupo dados os efeitos aleatórios. No modelo é possível considerar observações censuradas à direita Tipo I utilizando uma variável indicadora $\delta$ definida como segue:

$$
\delta_{i j}= \begin{cases}0, & \text { se } y_{i j} \text { é censurada, } \\ 1, & \text { se } y_{i j} \text { não é censurada. }\end{cases}
$$

Assim, a função densidade condicional para o vetor de observações $\boldsymbol{y}_{i}$ do $i$-ésimo grupo, 
dado $\boldsymbol{u}_{i}=\left(u_{1 i}, u_{2 i}\right)^{\top}$, é dada por:

$$
\begin{aligned}
P\left(\boldsymbol{y}_{i} \mid \boldsymbol{u}_{i}\right) & =\prod_{j=1}^{n_{i}}\left[f_{Y}\left(y_{i j} \mid \boldsymbol{u}_{i}\right)\right]^{\delta_{i j}}\left[S\left(y_{i j} \mid \boldsymbol{u}_{i}\right)\right]^{1-\delta_{i j}} \\
& =\prod_{j=1}^{n_{i}}\left[\frac{\sigma_{i j}}{\kappa_{i j}}\left(\frac{y_{i j}}{\kappa_{i j}}\right)^{\sigma_{i j}-1} \exp \left(-\left(\frac{y_{i j}}{\kappa_{i j}}\right)^{\sigma_{i j}}\right)\right]^{\delta_{i j}}\left[\exp \left(-\left(\frac{y_{i j}}{\kappa_{i j}}\right)^{\sigma_{i j}}\right)\right]^{1-\delta_{i j}} \\
& =\prod_{j=1}^{n_{i}}\left[\frac{\sigma_{i j}}{\kappa_{i j}}\left(\frac{y_{i j}}{\kappa_{i j}}\right)^{\sigma_{i j}-1}\right]^{\delta_{i j}} \exp \left(-\left(\frac{y_{i j}}{\kappa_{i j}}\right)^{\sigma_{i j}}\right) \\
& =\exp \sum_{j=1}^{n_{i}}\left[\delta_{i j} \log \left(\sigma_{i j} \kappa_{i j}^{-\sigma_{i j}} y_{i j}^{\sigma_{i j}-1}\right)-\kappa_{i j}^{-\sigma_{i j}} y_{i j}^{\sigma_{i j}}\right] \\
& =\exp \sum_{j=1}^{n_{i}}\left[\delta_{i j} \log \left(\sigma_{i j} \alpha_{i j} y_{i j}^{\sigma_{i j}-1}\right)-\alpha_{i j} y_{i j}^{\sigma_{i j}}\right] \\
& =\exp \left[\boldsymbol{\delta}_{i}^{\top}\left(\log \left(\boldsymbol{\sigma}_{i}\right)+\log \left(\boldsymbol{\alpha}_{i}\right)+\operatorname{diag}\left\{\boldsymbol{\sigma}_{i}-\mathbf{1}_{n_{i}}\right\} \log \left(\boldsymbol{y}_{i}\right)\right)-\boldsymbol{\alpha}_{i}^{\top} \boldsymbol{y}_{i}^{\star}\right] \\
& =\exp \left[g\left(\boldsymbol{y}_{i}, \boldsymbol{\delta}_{i}, \boldsymbol{u}_{i}\right)\right],
\end{aligned}
$$

em que

$$
\begin{aligned}
\alpha_{i j} & =\kappa_{i j}^{-\sigma_{i j}}, \\
\boldsymbol{\alpha}_{i} & =\left(\alpha_{i 1}, \alpha_{i 2}, \ldots, \alpha_{i n_{i}}\right)^{\top}, \\
\boldsymbol{\delta}_{i} & =\left(\delta_{i 1}, \delta_{i 2}, \ldots, \delta_{i n_{i}}\right)^{\top}, \\
\log \left(\boldsymbol{y}_{i}\right) & =\left(\log \left(y_{i 1}\right), \log \left(y_{i 2}\right), \ldots, \log \left(y_{i n_{i}}\right)\right)^{\top}, \\
\boldsymbol{y}_{i}^{\star} & =\left(y_{i 1}^{\sigma_{i 1}}, y_{i 2}^{\sigma_{i 2}}, \ldots, y_{i n_{i}}^{\sigma_{i n}}\right)^{\top},
\end{aligned}
$$

$\operatorname{com} g(\cdot)$ definida como

$$
g\left(\boldsymbol{y}_{i}, \boldsymbol{\delta}_{i}, \boldsymbol{u}_{i}\right)=\boldsymbol{\delta}_{i}^{\top}\left(\log \left(\boldsymbol{\sigma}_{i}\right)+\log \left(\boldsymbol{\alpha}_{i}\right)+\operatorname{diag}\left\{\boldsymbol{\sigma}_{i}-\mathbf{1}_{n_{i}}\right\} \log \left(\boldsymbol{y}_{i}\right)\right)-\boldsymbol{\alpha}_{i}^{\top} \boldsymbol{y}_{i}^{\star} .
$$

A função densidade conjunta de $\boldsymbol{y}_{i}$ e $\boldsymbol{u}_{i}=\left(u_{i 1}, u_{i 2}\right)^{\top}$ é dada por

$$
P\left(\boldsymbol{y}_{i}, \boldsymbol{u}_{i}\right)=P\left(\boldsymbol{y}_{i} \mid \boldsymbol{u}_{i}\right) f\left(u_{i 1} \mid \tau_{1}\right) f\left(u_{i 2} \mid \tau_{2}\right),
$$

em que $f(u \mid \tau)$ corresponde à função de densidade log-gama, dada por

$$
f(u \mid \tau)=\frac{(1 / \tau)^{1 / \tau}}{\Gamma(1 / \tau)} e^{\left(u-e^{u}\right) / \tau}
$$

A função densidade marginal para o vetor $\boldsymbol{y}_{i}$ é obtida integrando (4.5) sobre os efeitos 
aleatórios $\boldsymbol{u}_{i}$ e é dada pela seguinte expressão:

$$
P\left(\boldsymbol{y}_{i}\right)=\iint_{\Re^{2}} P\left(\boldsymbol{y}_{i}, \boldsymbol{u}_{i}\right) d u_{1 i} d u_{2 i}=\iint_{\Re^{2}} P\left(\boldsymbol{y}_{i} \mid \boldsymbol{u}_{i}\right) f\left(u_{i 1} \mid \tau_{1}\right) f\left(u_{i 2} \mid \tau_{2}\right) d u_{1 i} d u_{2 i} .
$$

Como no caso do modelo Weibull com efeitos aleatórios normal, a integral anterior não tem forma fechada e é preciso usar um método para aproximá-la. Neste trabalho foi aplicada a metodologia proposta por Liu e Yu (2008). Estes autores propuseram uma reformulação da função verossimilhança no caso de ter efeitos aleatórios não normais. O método baseia-se em multiplicar e dividir a função densidade conjunta pela função densidade de uma normal padrão $(\Phi)$ para assim poder aproximar a expressão resultante por meio da quadratura de Gauss-Hermite (QGH) em suas versões adaptativa ou não adaptativa. Uma explicação mais detalhada e com exemplos do método QGH pode ser encontrada no Capítulo 7. A expressão (4.6) reformulada passa a ser

$$
P\left(\boldsymbol{y}_{i}\right)=\iint_{\Re^{2}} P\left(\boldsymbol{y}_{i} \mid \boldsymbol{u}_{i}\right) \frac{f\left(u_{i 1} \mid \tau_{1}\right) f\left(u_{i 2} \mid \tau_{2}\right)}{\Phi\left(u_{i 1}\right) \Phi\left(u_{i 2}\right)} \Phi\left(u_{i 1}\right) \Phi\left(u_{i 2}\right) d u_{1 i} d u_{2 i}
$$

Usando $N_{Q G H}$ pontos de quadratura para cada uma das variáveis $u_{i 1}$ e $u_{i 2}$, a integral anterior pode ser aproximada por meio da expressão

$$
P\left(\boldsymbol{y}_{i}\right) \approx \sum_{l=1}^{N_{Q G H}} \sum_{m=1}^{N_{Q G H}} P\left(\boldsymbol{y}_{i} \mid\left(p_{l}, p_{m}\right)^{\top}\right) \frac{f\left(p_{l} \mid \tau_{1}\right) f\left(p_{m} \mid \tau_{2}\right)}{\Phi\left(p_{l}\right) \Phi\left(p_{m}\right)} w_{l} w_{m}
$$

em que $p_{l}$ e $p_{m}$ correspondem aos pontos de quadratura, enquanto $w_{l}$ e $w_{m}$ correspondem aos pesos.

Seja $\boldsymbol{\theta}=\left(\boldsymbol{\beta}_{1}^{\top}, \boldsymbol{\beta}_{2}^{\top}, \tau_{1}, \tau_{2}\right)^{\top}$ o vetor de parâmetros do modelo, que pertence ao espaço paramétrico $\Theta$, em que $\Theta=\Re^{p+q} \times \Re_{+}^{2}$, com $p$ e $q$ correspondendo ao número de efeitos fixos em $\mu$ e $\sigma$, respectivamente. Assim, a função de log-verossimilhança $l(\boldsymbol{\theta})$ para o modelo é dada por

$$
l(\boldsymbol{\theta})=\sum_{i=1}^{N} \log P\left(\boldsymbol{y}_{i}\right)
$$

A estimativa $\hat{\boldsymbol{\theta}}$ para o vetor de parâmetros $\boldsymbol{\theta}$ é obtida maximizando a função de log-verossimilhança $l(\boldsymbol{\theta})$ da expressão (4.9) no espaço paramétrico $\boldsymbol{\Theta}$. Neste trabalho foi construído um pacote específico denominado 'wlmm' no R, o qual dispõe da função wlmmML para obter as estimativas dos parâmetros do modelo, predição dos efeitos aleatórios e análise residual. Uma descrição das principais funções do pacote pode ser encontrada no Capítulo 8. 


\subsection{Predição dos efeitos aleatórios e proposta de resíduos}

Para este modelo com resposta Weibull e interceptos aleatórios log-gama é possível predizer os efeitos aleatórios usando o enfoque do melhor estimador apresentado na Seção 3.4. Da mesma forma, é possível usar para este modelo os resíduos $r_{o}, r_{d}$ e $r_{q}$ apresentados na Seção 3.5.

\subsection{Aplicações}

Nesta seção apresenta-se uma aplicação do método desenvolvido neste capítulo. Esta é realizada usando uma base de dados simulada, na qual as componentes do vetor de estimativas $\boldsymbol{\theta}$ são conhecidas.

\subsubsection{Exemplo com base de dados simulada}

Para o exemplo é usada uma base de dados que contem 100 observações sem censura divididas em $N=10$ grupos e $n_{i}=10$ número de observações por grupo. Para cada observação tem-se a variável resposta $y$ e duas variáveis explicativas $x_{1}$ e $x_{2}$. O modelo usado para a geração dos dados foi o seguinte:

$$
\begin{aligned}
y_{i j} \mid u_{1 i}, u_{2 i} & \stackrel{i n d}{\sim} W E I 3\left(\mu_{i j}, \sigma_{i j}\right), \\
\log \left(\mu_{i j}\right) & =1+2 x_{1}+u_{1 i} \\
\text { e } \quad \log \left(\sigma_{i j}\right) & =1-2 x_{2}+u_{2 i}, \quad i=1,2, \ldots, 10 \quad j=1,2, \ldots, 10,
\end{aligned}
$$

em que $u_{1 i} \sim L G(0,5), u_{2 i} \sim L G(0,5), x_{1} \sim N\left(2 ; 0,2^{2}\right)$ e $x_{2} \sim N\left(-1 ; 0,2^{2}\right)$. Assim, tem-se que o vetor verdadeiro de parâmetros é $\boldsymbol{\theta}=(1 ; 2 ; 1 ;-2 ; 0,5 ; 0,5)^{\top}$. Os dados são apresentados na Tabela B.2 do Apêndice B e na Figura 4.1 apresenta-se a densidade e o gráfico de caixa para a variável $y$.
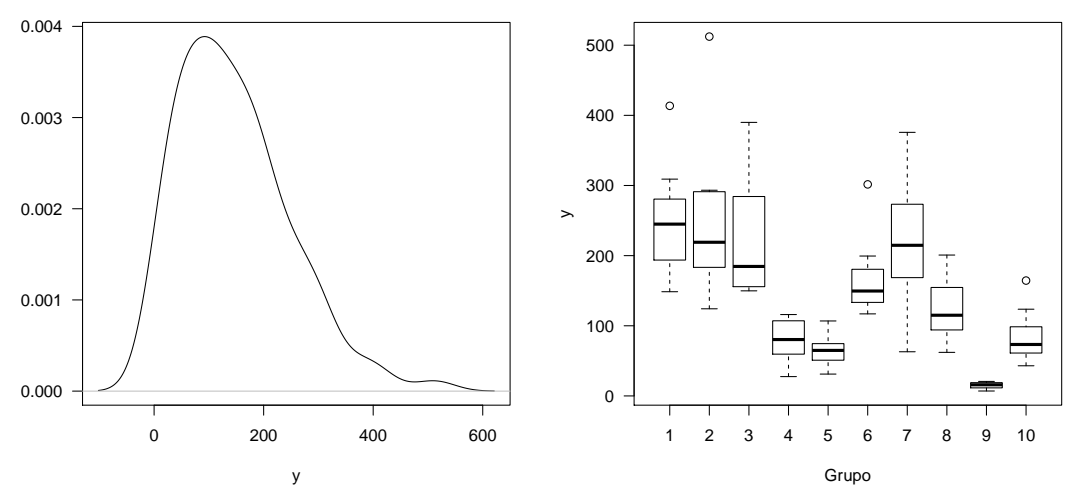

Figura 4.1: Função densidade e gráfico de caixa para a variável resposta y da base de dados simulada com resposta Weibull e efeitos aleatórios log-gama. 
No ajuste do modelo foi usada a QGH em suas versões adaptativa e não adaptativa com 15 pontos de quadratura e os resultados obtidos do ajuste são apresentados na Tabela 4.1. Dessa tabela observa-se que as estimativas $\hat{\boldsymbol{\theta}}$ com as duas versões (adaptativa e não adaptativa) são próximas do verdadeiro valor $\boldsymbol{\theta}$.

Tabela 4.1: Estimativas e erros padrão (em parênteses) dos parâmetros usando a quadratura GaussHermite adaptativa e não adaptativa para o modelo da base de dados simulada com resposta Weibull e efeitos aleatórios log-gama.

\begin{tabular}{cccc}
\hline Parâmetro & Valor real & Não adaptativa & Adaptativa \\
\hline$\beta_{11}$ & 1,0 & $1,26(0,01)$ & $1,28(0,01)$ \\
$\beta_{12}$ & 2,0 & $2,02(0,01)$ & $2,03(0,01)$ \\
$\beta_{21}$ & 1,0 & $0,16(0,35)$ & $0,36(0,30)$ \\
$\beta_{22}$ & $-2,0$ & $-1,05(0,33)$ & $-1,27(0,21)$ \\
\hline$\tau_{1}$ & 0,5 & $0,88(0,19)$ & $0,88(0,19)$ \\
$\tau_{2}$ & 0,5 & $0,83(0,19)$ & $1,05(0,50)$ \\
\hline
\end{tabular}

Uma das suposições do modelo estudado é que os efeitos aleatórios têm distribuição log-gama. Portanto, construiu-se a Figura 4.2, na qual os efeitos aleatórios preditos $\tilde{u}_{1}$ e $\tilde{u}_{2}$ foram plotados contra os quantis de uma distribuição log-gama com parâmetros $\hat{\tau}_{1}$ e $\hat{\tau}_{2}$ do ajuste QGH adaptativo dados na Tabela 4.1. Observa-se que a maioria pontos nos dois paineis estão próximos da reta identidade, isto indica que os efeitos aleatórios preditos não se afastam da distribuição log-gama.

$\tilde{u}_{1}$

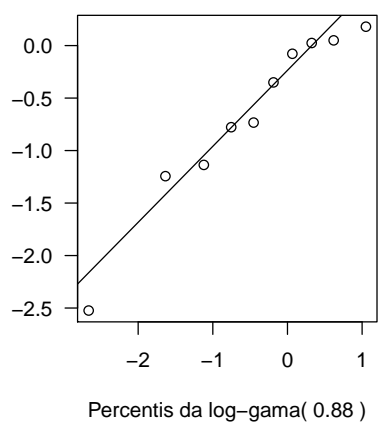

$\tilde{\mathrm{u}}_{2}$

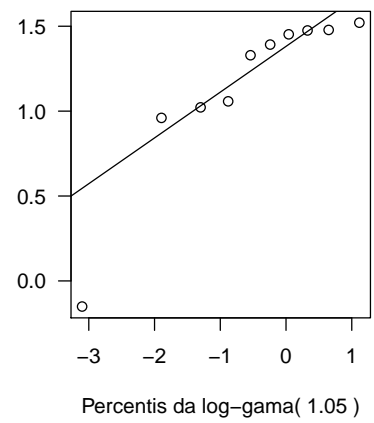

Figura 4.2: Gráfico de probabilidade dos efeitos aleatórios preditos $\tilde{u}_{1}$ e $\tilde{u}_{2}$ para a base de dados simulada com resposta Weibull e efeitos aleatórios log-gama.

Na Figura 4.3 apresentam-se os gráficos normais de probabilidade para os resíduos $r_{o}, r_{d}$ e $r_{q}$. Em cada um dos paneis foi incluído o valor-p do teste Anderson-Darling para avaliar a suposição de normalidade. Do gráfico e dos valores-p observa-se que só os resíduos $r_{d}$ e $r_{q}$ seguem uma distribuição normal.

Nesse exemplo a base de dados foi simulada com uma estrutura apropriada ao modelo aqui proposto e com vetor de parâmetros conhecidos. Dos resultados observa-se que as estimativas para $\boldsymbol{\theta}$, usando a QGH e QGHA, ficaram próximas do verdadeiro vetor de parâmetros. Além 

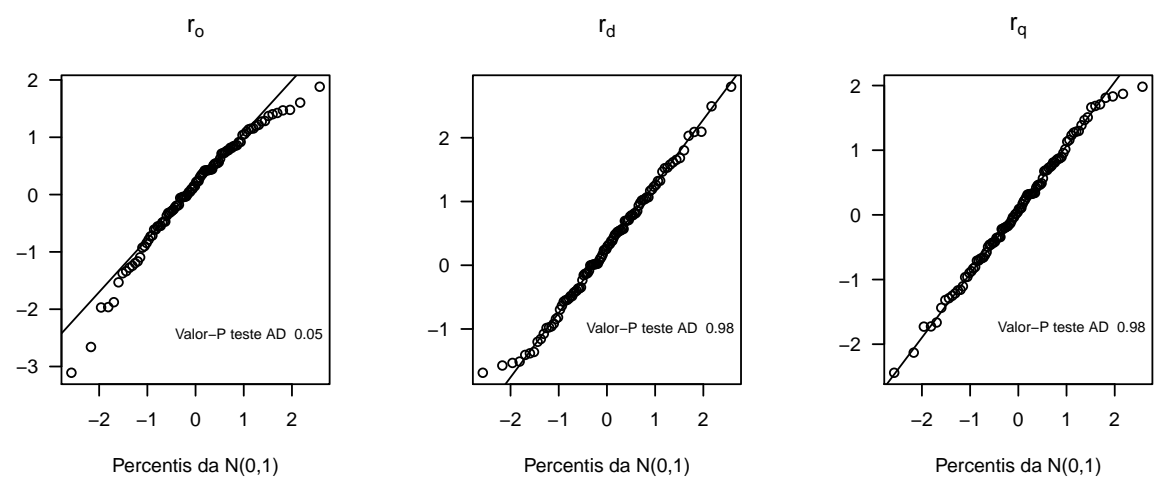

Figura 4.3: Gráficos normais de probabilidade dos resíduos $r_{o}, r_{d}$ e $r_{q}$ para base de dados simulada com resposta Weibull e efeitos aleatórios log-gama.

disso, nota-se que a distribuição dos efeitos aleatórios preditos coincidiu com a distribuição assumida log-gama. No entanto, McCulloch e Neuhaus (2011b) afirmam que às vezes a forma da distribuição dos efeitos aleatórios preditos não corresponde com a distribuição assumida para eles, mas que isso não se traduz necessariamente em um insatisfatorio desempenho do processo de estimação.

\subsection{Estudo de simulação}

Nesta seção apresenta-se um estudo de simulação Monte Carlo que tem como objetivo analisar o modelo proposto, em particular, examinar o desempenho do processo de estimação dos parâmetros, a distribuição das componentes de $\hat{\boldsymbol{\theta}}$ e a distribuição empírica dos resíduos propostos. A estrutura do modelo usada no estudo para a geração das amostras foi a seguinte:

$$
\begin{aligned}
y_{i j} \mid u_{1 i}, u_{2 i} & \stackrel{i n d}{\sim} W \operatorname{EI} 3\left(\mu_{i j}, \sigma_{i j}\right), \\
u_{1 i} & \sim L G(0,5), \\
u_{2 i} & \sim L G(0,6), \\
\log \left(\mu_{i j}\right) & =-1+0,5 x_{1}+u_{1 i} \\
\text { e } \quad \log \left(\sigma_{i j}\right) & =1,3+0,3 x_{2}+u_{2 i}, \quad i=1,2, \ldots, N, \quad j=1,2, \ldots, n_{i}
\end{aligned}
$$

em que $x_{1} \sim N\left(2 ; 0,3^{2}\right)$ e $x_{2} \sim N\left(-1 ; 0,3^{2}\right)$. Assim, tem-se que o vetor de parâmetros $\boldsymbol{\theta}$ a estimar tem como valores verdadeiros $\boldsymbol{\theta}=(-1 ; 0,5 ; 1,3 ; 0,3 ; 0,5 ; 0,6)^{\top}$. Simularamse 10000 amostras com a estrutura (4.10) para cada cenário resultante da combinação de $N=5,10,25,50$ número de grupos, $n_{i}=3,5,10,15$ observações por grupo e percentagem de observações censuradas de 0\%, 20\% e 40\%. Para cada uma das amostras foi ajustado o modelo obtendo-se e armazenando-se o vetor de estimativas $\hat{\boldsymbol{\theta}}$ e os resíduos $r_{o}, r_{q}, r_{d}$ e $r_{M}$. 


\subsubsection{Processo de estimação}

Para estudar o desempenho do processo de estimação foi usado erro quadrático médio multivariado (EQMM) apresentado em (3.23). Na Tabela 4.2 apresentam-se as médias do EQMM para cada combinação de $N, n_{i}$ e diferentes percentagens de censura. Observa-se que à medida que $N$ cresce, a média do EQMM dimiui, o que significa que as estimativas de $\hat{\boldsymbol{\theta}}$ ficam mais próximas de $\boldsymbol{\theta}$ na medida que há mais grupos disponíveis. O padrão geral observado no EQMM ao aumentar $n_{i}$ foi similar ao anterior, embora ao passar de $n_{i}=10$ para $n_{i}=15$ houve um pequeno incremento. Por outro lado, as médias do EQMM tendem a aumentar à medida que a percentagem de obsevações censuradas aumentam. É importante destacar que os padrões observados nesse caso são similares aos apresentados na Tabela 3.1 do modelo Weibull com efeitos aleatórios normal.

Tabela 4.2: EQMM entre $\hat{\boldsymbol{\theta}}$ e $\boldsymbol{\theta}$ no estudo de simulação para o modelo Weibull log-gama.

\begin{tabular}{|c|c|c|c|c|}
\hline \multicolumn{5}{|c|}{$0 \%$ de observações censurada } \\
\hline$N$ & 3 & 5 & 10 & 15 \\
\hline 5 & 408,78 & 40,30 & 13,49 & 12,76 \\
\hline 10 & 42,87 & 7,51 & 7,75 & 10,74 \\
\hline 25 & 4,86 & 1,87 & 2,37 & 4,07 \\
\hline 50 & 1,55 & 0,83 & 1,44 & 2,68 \\
\hline \multicolumn{5}{|c|}{$20 \%$ de observações censuradas } \\
\hline$N$ & 3 & 5 & 10 & 15 \\
\hline 5 & 881,14 & 55,62 & 14,50 & 13,42 \\
\hline 10 & 45,44 & 11,90 & 8,17 & 11,02 \\
\hline 25 & 5,69 & 1,92 & 1,98 & 2,90 \\
\hline 50 & 2,00 & 0,91 & 1,14 & 1,72 \\
\hline \multicolumn{5}{|c|}{ 40\% de observações censuradas } \\
\hline & 3 & 5 & 10 & 15 \\
\hline 5 & 1719,69 & 97,62 & 18,74 & 14,89 \\
\hline 10 & 64,09 & 13,26 & 8,82 & 15,76 \\
\hline 25 & 7,03 & 2,83 & 2,38 & 3,18 \\
\hline 50 & 2,40 & 1,39 & 1,38 & 2,13 \\
\hline
\end{tabular}

\subsubsection{Distribuição empírica de cada uma das componentes}

Para analisar a distribuição das componentes de $\hat{\boldsymbol{\theta}}$ foi construída a Figura 4.4. Nessa figura apresentam-se as estimativas das funções densidades para cada uma das componentes do vetor $\hat{\boldsymbol{\theta}}$ em dois casos extremos, o primeiro com menor número de observações, $N=5$ e $n_{i}=5$, e o segundo com maior número de observações, $N=25$ e $n_{i}=10$. Da figura observase que a distribuição para cada estimador $\hat{\beta}_{11}, \hat{\beta}_{12}, \hat{\beta}_{21}$ e $\hat{\beta}_{22}$, torna-se simétrica e centrada 
no verdadeiro valor dado pelas linhas verticais tracejadas escuras. Além disso, nota-se que essas distribuições ficaram mais concentradas para o caso com maior número de observações. Em relação as componentes $\hat{\tau}_{1}$ e $\hat{\tau}_{2}$, nota-se que as modas das distribuições ficaram próximas dos verdadeiros valores dados pela linha vertical.


Figura 4.4: Função densidade estimada para as componentes de $\hat{\boldsymbol{\theta}}$ no modelo Weibull com efeitos aleatórios log-gama. A linha contínua corresponde ao caso de $N=5$ e $n_{i}=5$. A linha tracejada corresponde ao caso de $N=25$ e $n_{i}=10$. A linha tracejada vertical escura corresponde a cada um dos valores verdadeiros de $\boldsymbol{\theta}$.

\subsubsection{Distribuição empírica dos resíduos}

Para estudar a distribuição empírica dos resíduos foram construídos gráficos de normalidade para as médias dos quantis de cada um dos resíduos ordenados contra os quantis da distribuição normal padrão para algumas combinações de $N, n_{i}$; os resultados são apresentados nas Figuras 4.5 a 4.12 e pode-se comentar o seguinte:

- Das Figuras 4.5 e 4.6 nota-se que a distribuição para $r_{o}$ não foi normal devido a que os pontos associados não ficaram sobre a linha reta.

- Das Figuras 4.7 e 4.8 observa-se que os pontos associados para $r_{q}$, no caso de $0 \%$ e 
$20 \%$ de censura, ficaram próximos de uma linha reta à medida que $n_{i}$ aumenta. No caso de $40 \%$ de censura observa-se uma leve curvatura nos pontos quando $n_{i}=3$.

- Nas Figuras 4.9 e 4.10 observa-se que os pontos no caso sem censura ficaram em linha reta, no entanto, o aumento na censura provoca una curvatura perto do $r_{d}=0$, esta situação é evidente no caso de $n_{i}=15$.

- Como esperado, das Figuras 4.11 e 4.12 observa-se que a distribuição para $r_{M}$ é assimétrica com valor máximo em 1 .
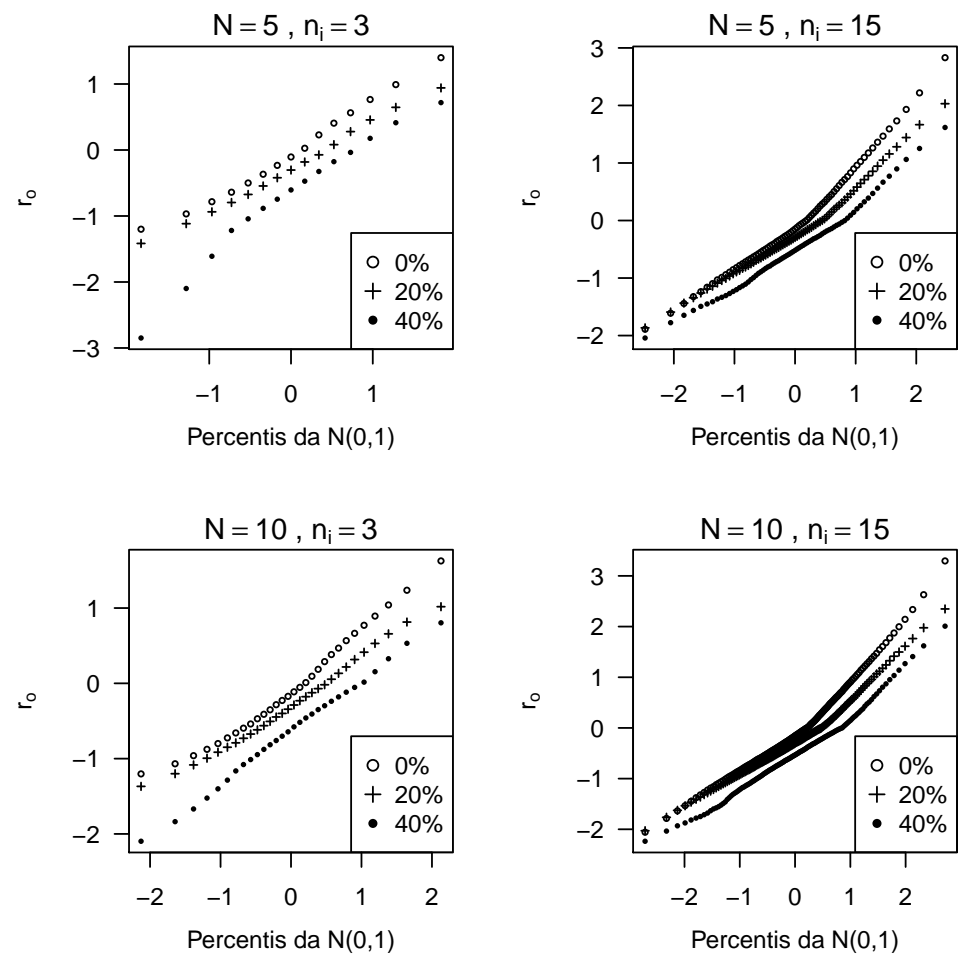

Figura 4.5: Gráfico normal de probabilidade para o resíduo $r_{o}$ no modelo Weibull log-gama com diferentes percentagens de censura para $N=5$ e $N=10$.

As medidas de média, desvio padrão, coeficiente de curtorse e coeficiente de asimetria foram obtidos para os resíduos $r_{o}, r_{q}$ e $r_{d}$ para estudar melhor a distribuição empírica e os resultados são apresentados nas Tabelas 4.3 a 4.5. Da Tabela 4.3 destaca-se que a média para $r_{o}$ tende a se afastar de 0 à medida que aumenta a percentagem de censura, enquanto o desvio padrão e curtose mantém-se ao redor de 0,73 e 3,18 respectivamente. Comportamento similar foi observado para a média de $r_{q}$ a partir da Tabela 4.4, o desvio padrão e a curtose ficaram ao redor de 0,83 e 2,56 respectivamente. Da Tabela 4.5 observa-se que a média de $r_{d}$ tende a 0 à medida que a percentagem de censura aumenta, o desvio padrão e a curtose para ficaram em torno de 0,93 e 2,36 respectivamente. Adicionalmente, observou-se que o coeficiente de assimetria ficou ao redor de 0 só para $r_{q}$ e $r_{d}$. 

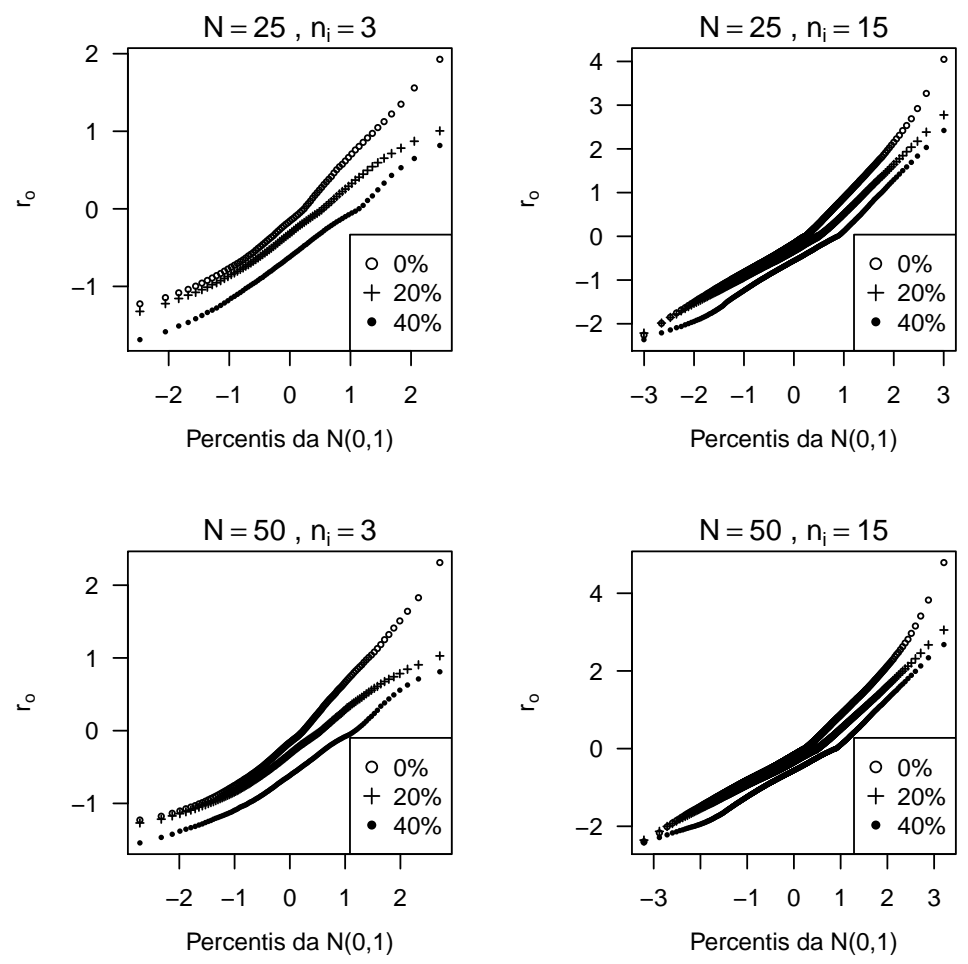

Figura 4.6: Gráfico normal de probabilidade para o resíduo $r_{o}$ no modelo Weibull log-gama com diferentes percentagens de censura para $N=25$ e $N=50$.

Dos comentários anteriores nota-se que o padrão observado é similar ao padrão das simulações da Seção 3.7.3 para o modelo Weibull com efeitos aleatórios normal. À medida que aumenta a censura, a média de $r_{q}$ tende a se afastar de 0 e o oposto para $r_{d}$. A distribuição empírica para os resíduos $r_{q}$ e $r_{d}$ é caracterizada por desvio padrão, curtose e assimetria em torno de 0,88, 2,46 e 0, respectivamente. Foi realizado também o teste de Shapiro-Wilk para os resíduos em cada combinação de $N, n_{i}$ e percentagem de censura. Obteve-se que a percentagem de rejeição da hipótese de normalidade foi $66 \%, 0 \%$ e $33 \%$ para $r_{o}, r_{q}$ e $r_{d}$ respectivamente. A partir desses resultados, pode-se concluir que a distribuição empírica observada para $r_{q}$ e $r_{d}$ é próxima da normal e assim estes podem ser usados para verificar se o modelo ajustado está correctamente ajustado. 

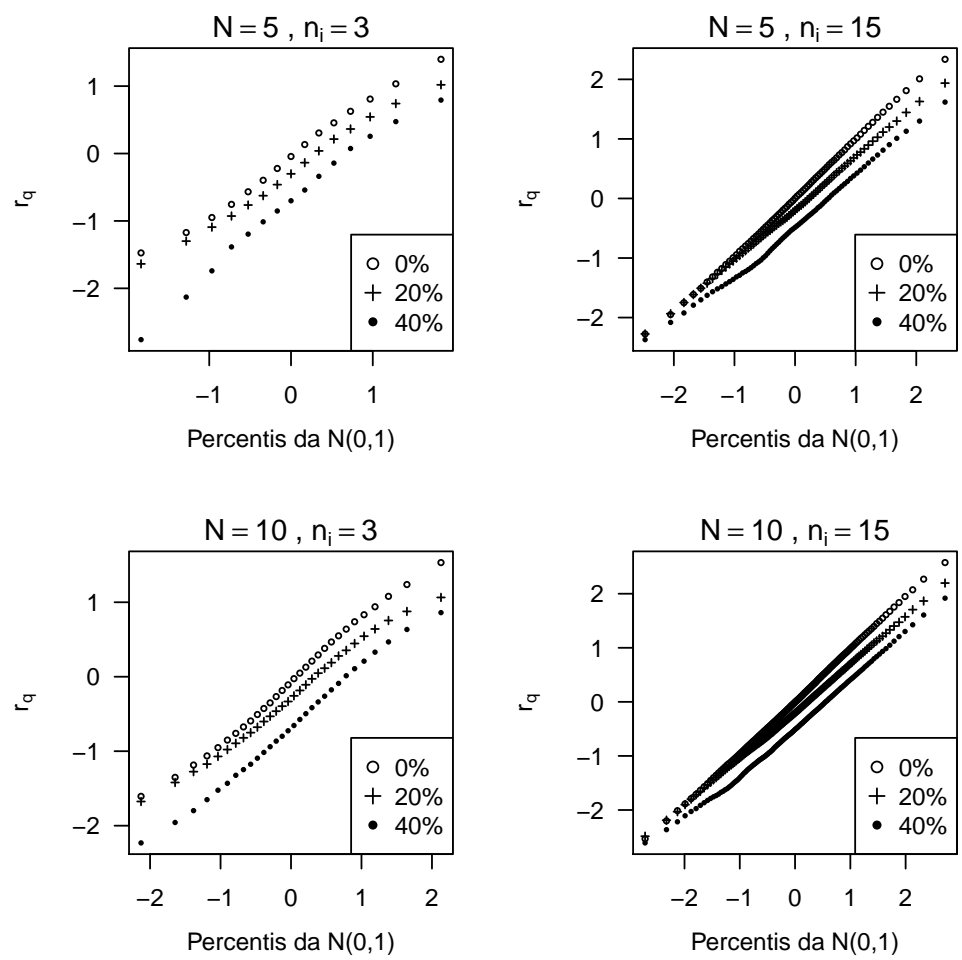

Figura 4.7: Gráfico normal de probabilidade para o resíduo $r_{q}$ no modelo Weibull log-gama com diferentes percentagens de censura para $N=5$ e $N=10$.

\subsection{Conclusões}

No presente capítulo foi exposto o modelo de dois níveis com resposta Weibull e interceptos aleatórios log-gama nos modelos dos parâmetros $\mu$ e $\sigma$ da distribuição, ou seja, consideraram-se efeitos aleatórios com distribuições diferente da normal. Os parâmetros do modelo foram obtidos pelo método de máxima verossimilhança e procedimentos computacionais especiais foram construídos para tal fim.

Para verificar a adequação desse novo modelo três tipos de resíduos foram propostos, o resíduo ordinario, o resíduo componente do desvio e o resíduo quantil normal aleatorizado; foi proposto também um procedimento para a predição dos efeitos aleatórios.

Um estudo de simulação Monte Carlo foi realizado com o intuito de examinar a distribuição empírica das componentes do vetor $\hat{\boldsymbol{\theta}}$ e a distribuição empírica dos resíduos propostos. Foi encontrado que, à medida que o número de observações aumenta, a distribuição das componentes de $\boldsymbol{\theta}$ fica mais concentrada no verdadeiro valor da componente. As distribuições empíricas dos resíduos Componente do Desvio e Quantil Normal Aleatorizado ficaram próximas da normal na medida em que o número de observações disponíveis aumentou. 

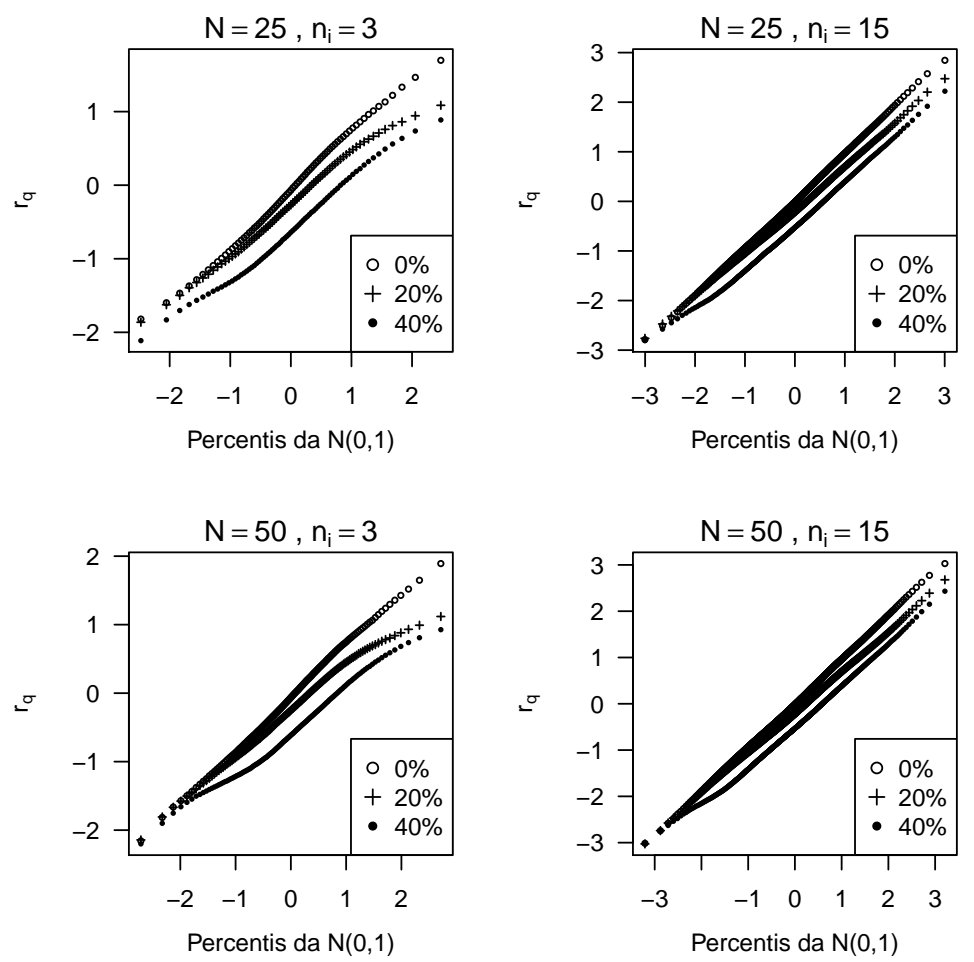

Figura 4.8: Gráfico normal de probabilidade para o resíduo $r_{q}$ no modelo Weibull log-gama com diferentes percentagens de censura para $N=25$ e $N=50$.
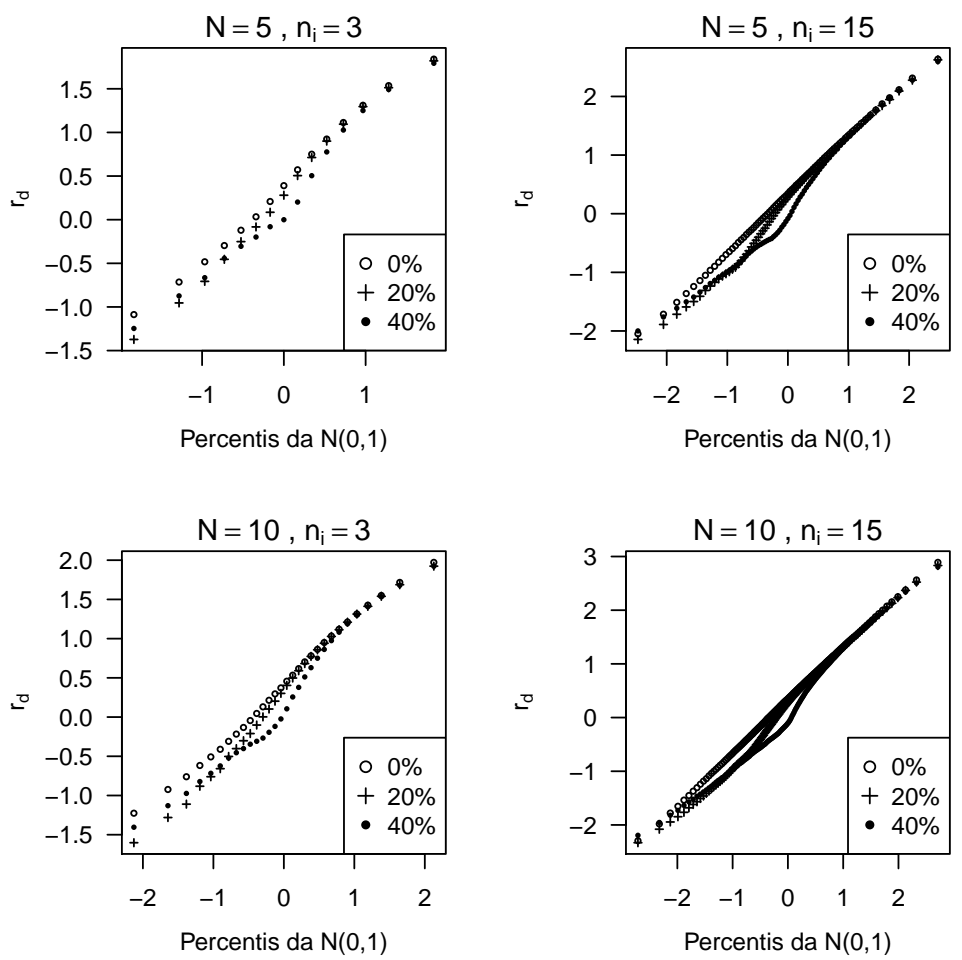

Figura 4.9: Gráfico normal de probabilidade para o resíduo $r_{d}$ no modelo Weibull log-gama com diferentes percentagens de censura para $N=5$ e $N=10$. 

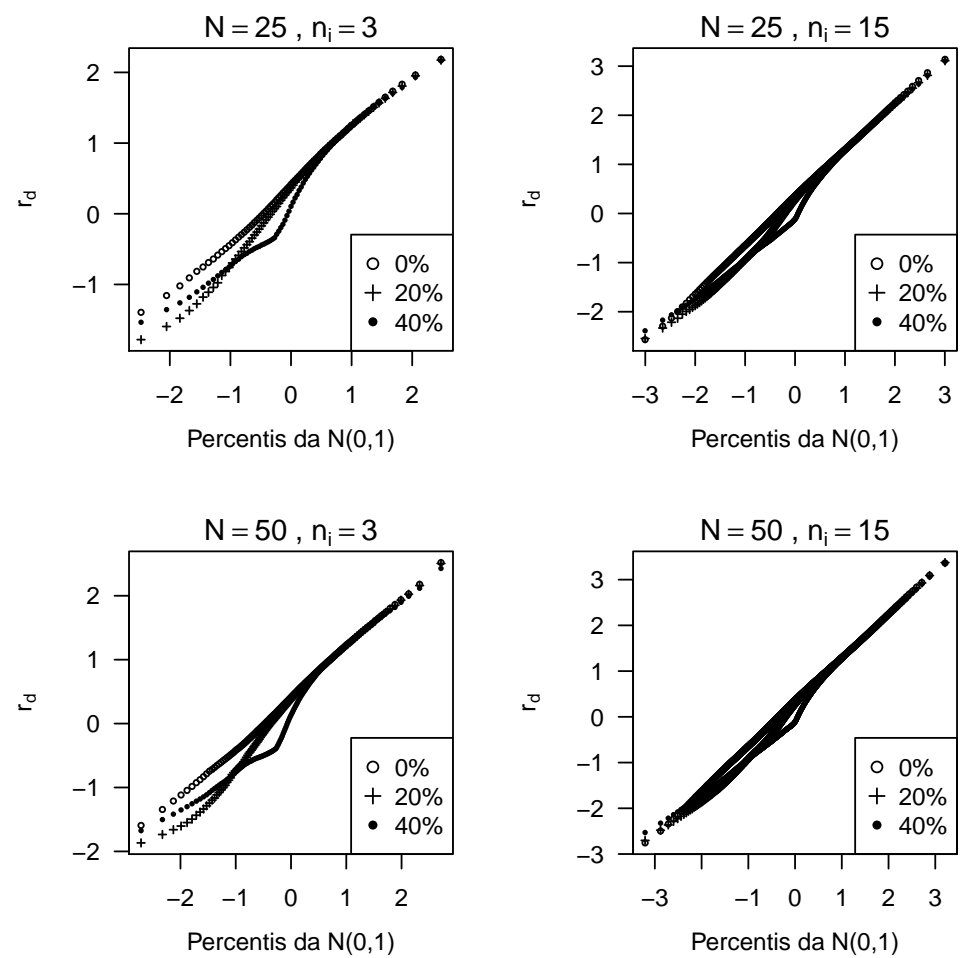

Figura 4.10: Gráfico normal de probabilidade para o resíduo $r_{d}$ no modelo Weibull log-gama com diferentes percentagens de censura para $N=25$ e $N=50$.
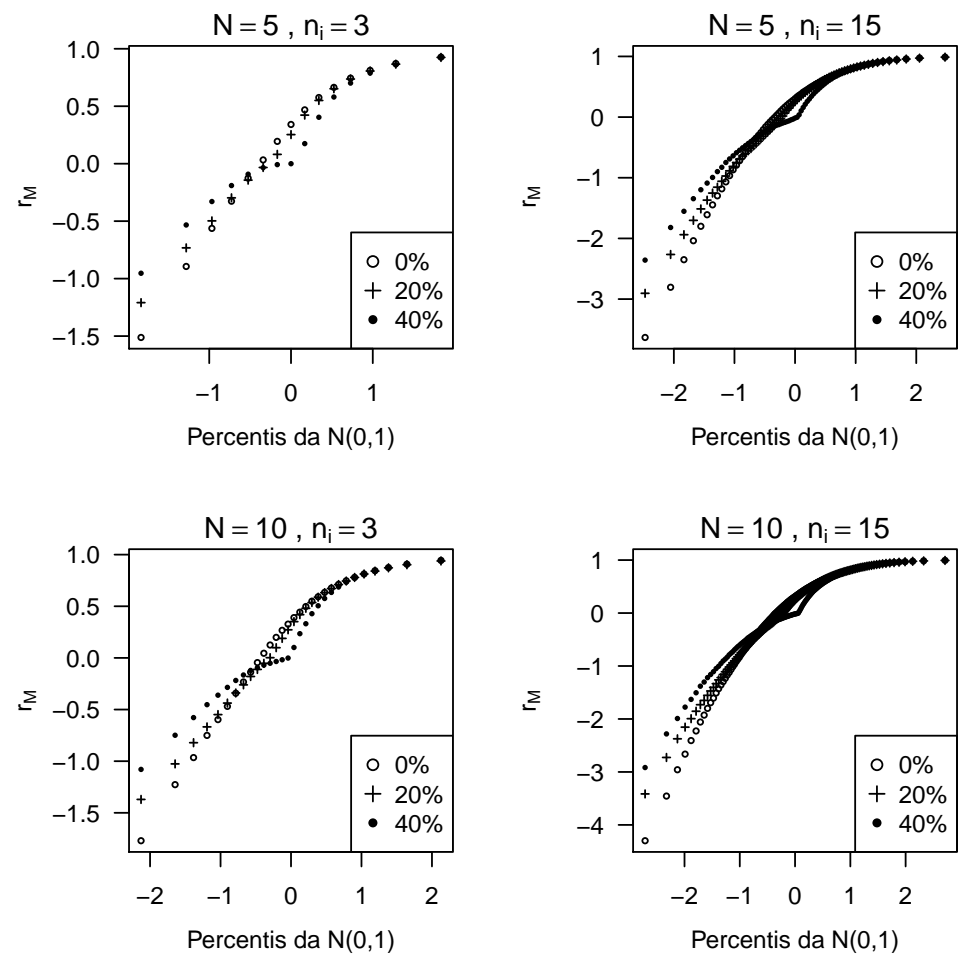

Figura 4.11: Gráfico normal de probabilidade para o resíduo $r_{M}$ no modelo Weibull log-gama com diferentes percentagens de censura para $N=5$ e $N=10$. 

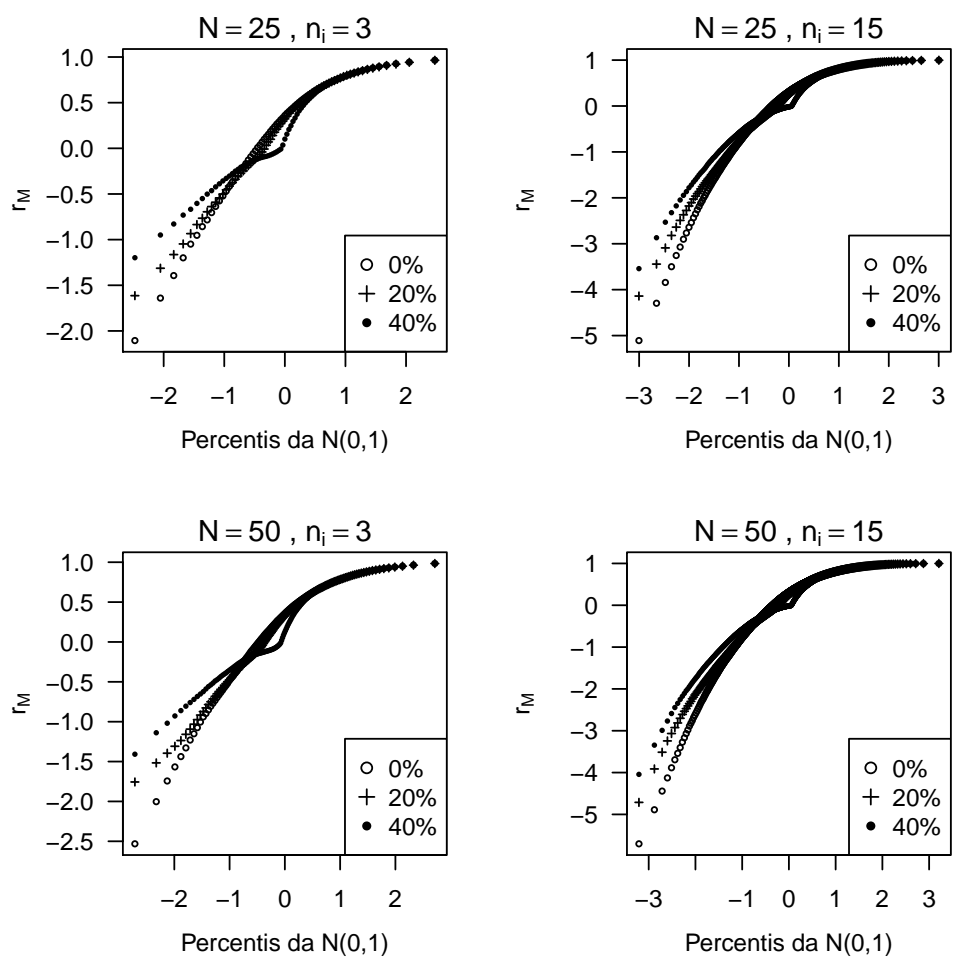

Figura 4.12: Gráfico normal de probabilidade para o resíduo $r_{M}$ no modelo Weibull log-gama com diferentes percentagens de censura para $N=25$ e $N=50$. 
Tabela 4.3: Média, desvio padrão, curtose e assimetria para $r_{o}$ no estudo de simulação do modelo Weibull log-gama com diferentes valores de $N, n_{i}$ e percentagem de censura.

\begin{tabular}{|c|c|c|c|c|c|c|}
\hline Censura & $N$ & $\overline{n_{i}}$ & Média & D.P. & Curtose & Assimetria \\
\hline \multirow{8}{*}{$0 \%$} & \multirow{2}{*}{5} & 3 & $-0,03$ & 0,75 & 2,16 & 0,26 \\
\hline & & 15 & $-0,00$ & 0,92 & 3,37 & 0,64 \\
\hline & \multirow{2}{*}{10} & 3 & $-0,04$ & 0,72 & 2,45 & 0,45 \\
\hline & & 15 & $-0,01$ & 0,91 & 3,76 & 0,74 \\
\hline & \multirow{2}{*}{25} & 3 & $-0,06$ & 0,70 & 2,79 & 0,58 \\
\hline & & 15 & $-0,01$ & 0,91 & 4,29 & 0,85 \\
\hline & \multirow{2}{*}{50} & 3 & $-0,06$ & 0,69 & 3,19 & 0,69 \\
\hline & & 15 & $-0,02$ & 0,90 & 4,74 & 0,92 \\
\hline \multirow{8}{*}{$20 \%$} & \multirow{2}{*}{5} & 3 & $-0,27$ & 0,67 & 2,18 & 0,13 \\
\hline & & 15 & $-0,21$ & 0,77 & 3,31 & 0,57 \\
\hline & \multirow{2}{*}{10} & 3 & $-0,27$ & 0,61 & 2,37 & 0,27 \\
\hline & & 15 & $-0,22$ & 0,77 & 3,60 & 0,64 \\
\hline & \multirow{2}{*}{25} & 3 & $-0,28$ & 0,55 & 2,37 & 0,29 \\
\hline & & 15 & $-0,23$ & 0,77 & 3,89 & 0,72 \\
\hline & \multirow{2}{*}{50} & 3 & $-0,29$ & 0,52 & 2,37 & 0,32 \\
\hline & & 15 & $-0,23$ & 0,77 & 3,98 & 0,72 \\
\hline \multirow{8}{*}{$40 \%$} & \multirow{2}{*}{5} & 3 & $-0,72$ & 0,95 & 2,94 & $-0,64$ \\
\hline & & 15 & $-0,48$ & 0,72 & 3,25 & 0,47 \\
\hline & \multirow{2}{*}{10} & 3 & $-0,64$ & 0,69 & 2,61 & $-0,08$ \\
\hline & & 15 & $-0,50$ & 0,75 & 3,59 & 0,46 \\
\hline & \multirow{2}{*}{25} & 3 & $-0,60$ & 0,55 & 2,67 & 0,29 \\
\hline & & 15 & $-0,52$ & 0,76 & 3,88 & 0,53 \\
\hline & \multirow{2}{*}{50} & 3 & $-0,58$ & 0,50 & 2,74 & 0,44 \\
\hline & & 15 & $-0,53$ & 0,76 & 4,02 & 0,55 \\
\hline
\end{tabular}

D.P. corresponde ao desvio padrão. 
Tabela 4.4: Média, desvio padrão, curtose e assimetria para $r_{q}$ no estudo de simulação do modelo Weibull log-gama com diferentes valores de $N, n_{i}$ e percentagem de censura.

\begin{tabular}{|c|c|c|c|c|c|c|}
\hline Censura & $N$ & $\overline{n_{i}}$ & Média & D.P. & Curtose & Assimetria \\
\hline \multirow{8}{*}{$0 \%$} & \multirow[b]{2}{*}{5} & 3 & $-0,05$ & 0,84 & 2,04 & 0,00 \\
\hline & & 15 & 0,01 & 0,97 & 2,65 & 0,03 \\
\hline & \multirow{2}{*}{10} & 3 & $-0,06$ & 0,81 & 2,20 & 0,03 \\
\hline & & 15 & 0,01 & 0,96 & 2,80 & 0,04 \\
\hline & \multirow{2}{*}{25} & 3 & $-0,06$ & 0,78 & 2,39 & $-0,00$ \\
\hline & & 15 & 0,01 & 0,96 & 2,91 & 0,04 \\
\hline & \multirow{2}{*}{50} & 3 & $-0,06$ & 0,78 & 2,62 & $-0,04$ \\
\hline & & 15 & 0,00 & 0,95 & 2,98 & 0,04 \\
\hline \multirow{8}{*}{$20 \%$} & \multirow{2}{*}{5} & 3 & $-0,29$ & 0,78 & 2,01 & $-0,01$ \\
\hline & & 15 & $-0,17$ & 0,86 & 2,75 & 0,05 \\
\hline & \multirow{2}{*}{10} & 3 & $-0,28$ & 0,72 & 2,15 & 0,00 \\
\hline & & 15 & $-0,18$ & 0,87 & 2,85 & 0,07 \\
\hline & \multirow{2}{*}{25} & 3 & $-0,27$ & 0,68 & 2,31 & $-0,11$ \\
\hline & & 15 & $-0,19$ & 0,87 & 2,95 & 0,08 \\
\hline & \multirow{2}{*}{50} & 3 & $-0,26$ & 0,66 & 2,52 & $-0,20$ \\
\hline & & 15 & $-0,19$ & 0,87 & 3,00 & 0,04 \\
\hline \multirow{8}{*}{$40 \%$} & \multirow{2}{*}{5} & 3 & $-0,75$ & 0,99 & 2,44 & $-0,37$ \\
\hline & & 15 & $-0,47$ & 0,85 & 2,56 & 0,16 \\
\hline & \multirow{2}{*}{10} & 3 & $-0,67$ & 0,79 & 2,25 & 0,01 \\
\hline & & 15 & $-0,49$ & 0,88 & 2,70 & 0,14 \\
\hline & \multirow{2}{*}{25} & 3 & $-0,61$ & 0,67 & 2,33 & 0,14 \\
\hline & & 15 & $-0,50$ & 0,88 & 2,79 & 0,16 \\
\hline & \multirow{2}{*}{50} & 3 & $-0,57$ & 0,63 & 2,45 & 0,18 \\
\hline & & 15 & $-0,51$ & 0,88 & 2,84 & 0,15 \\
\hline
\end{tabular}

D.P. corresponde ao desvio padrão. 
Tabela 4.5: Média, desvio padrão, curtose e assimetria para $r_{d}$ no estudo de simulação do modelo Weibull log-gama com diferentes valores de $N, n_{i}$ e percentagem de censura.

\begin{tabular}{|c|c|c|c|c|c|c|}
\hline Censura & $N$ & $n_{i}$ & Média & D.P. & Curtose & Assimetria \\
\hline \multirow{8}{*}{$0 \%$} & \multirow{2}{*}{5} & 3 & 0,40 & 0,86 & 2,04 & $-0,01$ \\
\hline & & 15 & 0,33 & 0,99 & 2,64 & $-0,05$ \\
\hline & \multirow{2}{*}{10} & 3 & 0,41 & 0,82 & 2,19 & $-0,04$ \\
\hline & & 15 & 0,33 & 0,98 & 2,79 & $-0,06$ \\
\hline & \multirow{2}{*}{25} & 3 & 0,41 & 0,80 & 2,39 & $-0,01$ \\
\hline & & 15 & 0,34 & 0,97 & 2,90 & $-0,06$ \\
\hline & \multirow{2}{*}{50} & 3 & 0,41 & 0,79 & 2,61 & 0,02 \\
\hline & & 15 & 0,34 & 0,97 & 2,96 & $-0,06$ \\
\hline \multirow{8}{*}{$20 \%$} & \multirow{2}{*}{5} & 3 & 0,08 & 0,82 & 2,01 & $-0,18$ \\
\hline & & 15 & 0,21 & 1,09 & 2,26 & $-0,06$ \\
\hline & \multirow{2}{*}{10} & 3 & 0,29 & 0,93 & 2,15 & $-0,20$ \\
\hline & & 15 & 0,21 & 1,08 & 2,38 & $-0,05$ \\
\hline & \multirow{2}{*}{25} & 3 & 0,29 & 0,93 & 2,31 & $-0,23$ \\
\hline & & 15 & 0,21 & 1,07 & 2,46 & $-0,07$ \\
\hline & \multirow{2}{*}{50} & 3 & 0,29 & 0,93 & 2,45 & $-0,23$ \\
\hline & & 15 & 0,21 & 1,07 & 2,53 & $-0,06$ \\
\hline \multirow{8}{*}{$40 \%$} & \multirow{2}{*}{5} & 3 & $-0,40$ & 0,46 & 2,33 & $-0,55$ \\
\hline & & 15 & 0,10 & 1,07 & 2,23 & 0,27 \\
\hline & \multirow{2}{*}{10} & 3 & 0,15 & 0,86 & 1,95 & 0,15 \\
\hline & & 15 & 0,10 & 1,06 & 2,32 & 0,27 \\
\hline & \multirow{2}{*}{25} & 3 & 0,20 & 0,92 & 1,96 & 0,18 \\
\hline & & 15 & 0,11 & 1,05 & 2,40 & 0,27 \\
\hline & \multirow{2}{*}{50} & 3 & 0,19 & 0,93 & 1,98 & 0,18 \\
\hline & & 15 & 0,11 & 1,05 & 2,45 & 0,28 \\
\hline
\end{tabular}

D.P. corresponde ao desvio padrão. 


\section{Capítulo 5}

\section{Extensões do modelo multinível Weibull}

Neste capítulo são expostas duas extensões para o modelo multinível Weibull apresentado nos capítulos anteriores. Na Seção 5.1 são apresentadas várias distribuições de probabilidade usadas para flexibilizar a distribuição dos interceptos aleatórios do modelo multinível Weibull. Na Seção 5.1 é mostrada a extensão do modelo multinível Weibull que considera interceptos e inclinações aleatórias com distribuição normal bivariada nos modelos dos parâmetros da distribuição Weibull. As duas extensões foram implementadas no pacote wlmmML descrito no Capítulo 8.

\subsection{Flexibilização da distribuição dos interceptos aleató- rios}

No Capítulo 3 foi apresentado o modelo Weibull de dois níveis com interceptos aleatórios seguindo distribuição normal e no Capítulo 4 foi relaxada essa suposição para a distribuição $\log$-gama. Nesses dois modelos a estimação dos parâmetros $\boldsymbol{\theta}$ é realizada maximizando a função de log-verossimilhança $l(\boldsymbol{\theta})$, que pode ser escrita de maneira geral como

$$
l(\boldsymbol{\theta})=\sum_{i=1}^{N} \log P\left(\boldsymbol{y}_{i}\right),
$$

em que $P\left(\boldsymbol{y}_{i}\right)$ corresponde à função densidade marginal do vetor de observações $\boldsymbol{y}_{i}=$ $\left(y_{i 1}, \ldots, y_{i n_{i}}\right)^{T}$ para o $i$-ésimo grupo e que é obtida calculando a integral

$$
P\left(\boldsymbol{y}_{i}\right)=\iint_{\Re^{2}} P\left(\boldsymbol{y}_{i} \mid \boldsymbol{u}_{i}\right) f\left(u_{i 1} \mid \tau_{1}\right) f\left(u_{i 2} \mid \tau_{2}\right) d u_{1 i} d u_{2 i},
$$

na qual $P\left(\boldsymbol{y}_{i} \mid \boldsymbol{u}_{i}\right)$ corresponde ao produto de funções densidades da Weibull avaliadas em cada um dos elementos do vetor de observações $\boldsymbol{y}_{i}$, enquanto $f\left(u_{i 1} \mid \tau_{1}\right)$ e $f\left(u_{i 2} \mid \tau_{2}\right)$ correspondem às funções densidade dos interceptos aleatórios. A integral da expressão (5.1), que é função dos interceptos aleatórios $u_{i 1}$ e $u_{i 2}$, é aproximada com a aplicação do método de qua- 
dratura de Gauss-Hermite (QGH) ou quadratura de Gauss-Hermite adaptativa (QGHA). No presente trabalho foi usada a reformulação da função de verossimilhança proposta por Liu e Yu (2008), a qual consiste básicamente em multiplicar e dividir pela densidade de uma distribuição normal para permitir o uso da QGH ou QGHA no caso em que a distribuição dos efeitos aleatórios não é normal.

A implementação computacional para ajustar este tipo de modelos foi realizada na linguagem R por meio da função wlmmML e que está descrita com detalhes no Capítulo 8. Nessa implementação foram consideradas mais duas distribuições para os efeitos aleatórios além da normal e log-gama. Essas distribuições foram a logística e a Cauchy. A escolha dessas distribuições foi baseada no trabalho de Broström e Holmberg (2011a) para modelos lineares generalizados mistos com intercepto aleatório. Na sequência apresentam-se as funções de densidade das distribuições normal, log-gama, logística e Cauchy que foram consideradas.

Distribuição normal:

$$
f(u \mid \tau)=\frac{1}{(2 \pi)^{1 / 2} \tau} e^{-\frac{u^{2}}{2 \tau^{2}}}
$$

com média 0 e variância $\tau^{2}$.

Distribuição log-gama:

$$
f(u \mid \tau)=\frac{(1 / \tau)^{1 / \tau}}{\Gamma(1 / \tau)} e^{\left(u-e^{u}\right) / \tau},
$$

em que $e^{u}$ segue distribuição gama com média 1 e variância $\tau$.

Distribuição logística:

$$
f(u \mid \tau)=\frac{e^{-u / \tau}}{\tau\left(1+e^{-u / \tau}\right)^{2}},
$$

em que $u$ tem média zero e variância $\pi^{2} \tau^{2} / 3$.

Distribuição Cauchy:

$$
f(u \mid \tau)=\frac{1}{\pi}\left(\frac{\tau}{u^{2}+\tau^{2}}\right) .
$$

Na Figura 5.1 apresentam-se quatro gráficos da função densidade para vários valores do parâmetro $\tau$.

\subsection{Generalização do modelo multinível Weibull}

No Capítulo 3 foi apresentado o modelo multinível com resposta Weibull considerando interceptos aleatórios distribuídos normais e log-gama nos modelos dos parâmetros da distribuição. Nesta seção é apresentada uma extensão desse modelo, a qual permite incluir tanto intercepto quanto inclinação aleatória nos modelos dos parâmetros da distribuição Weibull. 



Figura 5.1: Função de densidade dos efeitos aleatórios para diferentes valores do parâmetro $\tau$.

Suponha-se $y_{i j}$ como a $j$-ésima medida do $i$-ésimo grupo, com $i=1,2, \ldots, N$ número de grupos e $j=1,2, \ldots, n_{i}$ observações por grupo. Dados os vetores de efeitos aleatórios $\boldsymbol{u}_{1 i}$ e $\boldsymbol{u}_{2 i}$, a distribuição de $y_{i j}$ é dada por

$$
\begin{aligned}
y_{i j} \mid \boldsymbol{u}_{1 i}, \boldsymbol{u}_{2 i} & \stackrel{i n d}{\sim} W E I 3\left(\mu_{i j}, \sigma_{i j}\right), \\
\boldsymbol{u}_{1 i} & \stackrel{i i d}{\sim} N_{2}\left(\mathbf{0}, \boldsymbol{D}_{1}\right), \\
\boldsymbol{u}_{2 i} & \stackrel{i i d}{\sim} N_{2}\left(\mathbf{0}, \boldsymbol{D}_{2}\right),
\end{aligned}
$$

em que os parâmetros $\mu$ e $\sigma$ são modelados, respectivamente, por:

$$
\begin{aligned}
& \log \left(\mu_{i j}\right)=\boldsymbol{X}_{1 i, j}^{T} \cdot \boldsymbol{\beta}_{1}+\boldsymbol{Z}_{1 i, j}^{T} \cdot \boldsymbol{u}_{1 i}, \\
& \log \left(\sigma_{i j}\right)=\boldsymbol{X}_{2 i, j}^{T} \cdot \boldsymbol{\beta}_{2}+\boldsymbol{Z}_{2 i, j}^{T} \cdot \boldsymbol{u}_{2 i} .
\end{aligned}
$$

$\boldsymbol{X}_{1 i}$ e $\boldsymbol{X}_{2 i}$ são matrizes de planejamento conhecidas para o grupo $i$ e, $\boldsymbol{X}_{1 i, j}$. e $\boldsymbol{X}_{2 i, j}$. correspondem à $j$-ésima linha de $\boldsymbol{X}_{1 i}$ e $\boldsymbol{X}_{2 i}$ respectivamente; a mesma notação aplica-se às matrizes $\boldsymbol{Z}_{1 i}$ e $\boldsymbol{Z}_{2 i}$. Os vetores de parâmetros fixos desconhecidos a serem estimados são denotados por $\boldsymbol{\beta}_{1}$ e $\boldsymbol{\beta}_{2}$. Os vetores $\boldsymbol{u}_{1 i}$ e $\boldsymbol{u}_{2 i}$ correspondem aos efeitos aleatórios de média $\mathbf{0}$ e matriz de covariânzas $\boldsymbol{D}_{1}$ e $\boldsymbol{D}_{2}$, respectivamente.

A contribuição do vetor de observações $\boldsymbol{y}_{i}=\left(y_{i 1}, \ldots, y_{i n_{i}}\right)^{T}$ do $i$-ésimo grupo à função de verossimilhança é dado por

$$
P\left(\boldsymbol{y}_{i} \mid \boldsymbol{\beta}_{1}, \boldsymbol{\beta}_{2}, \boldsymbol{D}_{1}, \boldsymbol{D}_{2}\right)=\int_{\Re^{4}} \prod_{j=1}^{n_{i}} f_{Y}\left(y_{i j} \mid \boldsymbol{\beta}_{1}, \boldsymbol{\beta}_{2}\right) f\left(\boldsymbol{u}_{1 i} \mid \boldsymbol{D}_{1}\right) f\left(\boldsymbol{u}_{2 i} \mid \boldsymbol{D}_{2}\right) d \boldsymbol{u}_{1 i} d \boldsymbol{u}_{2 i}
$$


em que $f_{Y}(\cdot)$ corresponde à função densidade da distribuição Weibull e $f(\cdot)$ à função densidade da distribuição normal bivariada de média $\mathbf{0}$ avaliada no vetor de efeitos aleatórios. Para obter $P\left(\boldsymbol{y}_{i} \mid \boldsymbol{\beta}_{1}, \boldsymbol{\beta}_{2}, \boldsymbol{D}_{1}, \boldsymbol{D}_{2}\right)$ na expressão (5.4) é possivel usar o método QGH ou QGHA descritos na Seção 7.1. Sejam $\boldsymbol{D}_{1}^{*}$ e $\boldsymbol{D}_{2}^{*}$ os elementos únicos de $\boldsymbol{D}_{1}$ e $\boldsymbol{D}_{2}$, respectivamente. Assím, $\boldsymbol{\theta}$ é dado por $\boldsymbol{\theta}=\left(\boldsymbol{\beta}_{1}, \boldsymbol{\beta}_{2}, \boldsymbol{D}_{1}^{*}, \boldsymbol{D}_{2}^{*}\right)^{T}$ o vetor de parâmetros do modelo, a função de log-verossimilhança é escrita como

$$
l(\boldsymbol{\theta})=\sum_{i=1}^{N} \log P\left(\boldsymbol{y}_{i} \mid \boldsymbol{\theta}\right)
$$

O vetor de estimativas dos parâmetros $\boldsymbol{\theta}$ do modelo pode ser obtido maximizando a função de $\log$-verossimilhança $l(\boldsymbol{\theta})$ usando a função wlmmML. Detalhes do uso da função wlmmML são apresentados no Capítulo 8. Note-se que no cálculo de $l(\boldsymbol{\theta})$ é preciso solucionar $N$ integrais em quatro dimensões como as dadas na expressão (5.4), e para a obtenção das estimativas de $\boldsymbol{\theta}$ o cálculo de $l(\boldsymbol{\theta})$ é repetido muitas vezes, por tanto, o procedimento é computacionalmente intensivo e demorado ainda com a QGH.

\subsection{Conclusões}

Neste capítulo apresentaram-se duas extensões ao modelo multinível Weibull: interceptos aleatórios com distribuição logística e Cauchy e, interceptos e inclinações aleatórias com distribuição normal multivariada. Um procedimiento computacional específico foi realizado para a análise desse novo tipo de modelos, que está incluso na função wlmmML explicada no Capítulo 8. Com estas extensões realizadas se enriquece os modelos multinível Weibull ao permitir que o pesquisador tenha disponíveis várias distribuições para os efeitos aletórios, como também a posibilidade de incluir interceptos e inclinações aleatórias distribuídas normalmente nos modelos dos parâmetros da distribuição da variável resposta.

Nota-se que para os modelos apresentados neste capítulo a decisão de incluir ou não um efeito aleatório poderia ter tomada também utilizando a estatística da razão de verossimilhanças, mas são necessários novos estudos de simulação para determinar a distribuição assintótica desta estatística. Outra opção mais simples e factível de ser realizada com a metodologia proposta é a construção de intervalos de confiança para as componentes de variância. 


\section{Capítulo 6}

\section{Impacto de uma incorreta especificação na distribuição dos efeitos aleatórios}

Os modelos lineares generalizados mistos (MLGM) propostos por Breslow e Clayton (1993) têm sido uma ferramenta muito usada para a análise de dados longitudinais e dados agrupados. No entanto, esta classe de modelos tem a forte suposição de que a distribuição de efeitos aleatórios é normal por conveniência computacional, mas a suposição de normalidade pode ser irrealista em algumas aplicações (Huang, 2009). Uma vez que os efeitos aleatórios não são observados, é difícil verificar esta suposição e caso, a verdadeira distribuição dos efeitos aleatórios seja muito diferente da normal, as estimativas e inferências podem ser seriamente afetadas. Nos últimos anos foram desenvolvidas pesquisas para avaliar o impacto de assumir a distribuição normal para efeitos aleatórios, quando na verdade a distribuição subjacente não é normal, sobre o comportamento das estimativas dos parâmetros e no desempenho dos procedimentos de inferência. Esse tipo de trabalho é denominado estudo sobre o impacto de uma incorreta especificação da distribuição dos efeitos aleatórios. Nesses estudos as covariáveis de interesse têm sido classificadas em duas classes de covariáveis. A primeira corresponde à covariável intergrupal, a qual é constante para as observações do mesmo grupo, mas diferente para observações de grupos diferentes. A segunda corresponde à covariável intragrupal a qual varea em cada grupo, mas que tem uma média constante entre grupos (McCulloch e Neuhaus, 2011a). O interesse dos estudos de incorreta especificação tem sido avaliar o impacto nas estimativas do intercepto, dos coeficientes das covariáveis intergrupal e intragrupal, nas estimativas das componentes de variância, nas predições e na distribuição dos efeitos aleatórios, assim como nos testes de hipóteses.

Nos modelos lineares mistos (MLM) Verbeke e Lesaffre (1997) mostraram que os estimadores de máxima verossimilhança dos efeitos fixos e das componentes de variância são consistentes e assintoticamente normais, ainda que a distribuição dos efeitos aletórios não seja normal. Eles realizaram um estudo de simulação assumindo efeitos aleatórios normais, mas gerando o intercepto e a inclinação aletória de cinco distribuições diferentes: normal, 
mistura simétrica e assimétrica de duas normais, log-normal e discreta. Usando a distância relativa eles mostraram uma consistência clara dos estimadores de máxima verossimilhança com o aumento do tamanho total da amostra. Desafortunadamente, esses mesmos resultados conclusivos não foram encontrados para os MLGM's.

Para o modelo logístico misto (MLogM), Neuhaus et al. (1992) realizaram um estudo de simulação no qual foram consideradas três distribuições para os efeitos aleatórios: gama, t-Student e normal. Eles obtiveram as estimativas dos parâmetros assumindo uma distribuição normal para os efeitos aleatórios e verificaram que as estimativas dos parâmetros do modelo são assintoticamente viesadas mas que a magnitude do viés é relativamente pequena.

Heagerty e Kurland (2001) estudaram o MLogM por meio de um estudo de simulação no qual o efeito aleatório seguiu as seguintes quatro situações: intercepto aleatório distribuído não normal, variância do intercepto aleatório dependendo de uma covariável intergrupal, assumir intercepto aleatório quando de fato há intercepto e inclinação aleatórios, e interceptos aleatórios autocorrelacionados. Através do estudo de simulação, utilizando o viés relativo, eles encontraram pouco viés nas estimativas dos parâmetros para o modelo marginal.

Agresti et al. (2004) estudaram três modelos diferentes (MLogM, um modelo de razão de chances com efeitos aleatórios e um modelo de fragilidade) através de estudo de simulação e, usando a distância absoluta, mostraram que uma considerável perda de eficiência na estimação dos parâmetros é obtida ao assumir uma distribuição paramétrica para os efeitos aleatórios que difere da verdadeira distribuição.

Por meio de simulações Litière et al. (2007) estudaram o impacto de uma incorreta especificação da distribuição dos efeitos aleatórios na potência do teste de Wald para testar a significância de efeitos fixos em um MLogM. Para a geração dos efeitos aleatórios eles consideraram quatro distribuições normal, potência (Johnson et al., 1994), discreta e mistura assimétrica de duas normais. A estimação dos parâmetros do modelo foi estudada assumindo normalidade para os efeitos aleatórios. Esses autores afirmaram que a especificação incorreta da distribuição dos efeitos aleatórios pode produzir um aumento significativo no erro tipo II do teste de Wald. Para o mesmo MLogM, Litière et al. (2008) estudaram o impacto da incorreta especificação mediante simulações baseadas em um estudo sobre esquizofrenia no qual foram consideradas nove distribuições para a geração dos efeitos aleatórios. Assumindo normalidade para os efeitos aleatórios, eles encontraram que as estimativas para as componentes de variância são severamente afetadas e que os coeficientes associados às covariáveis intragrupal são menos afetadas quando a variância dos efeitos aleatórios é menor ou igual a 4. Adicionalmente, eles encontraram que a incorreta especificação afeta severamente a potência do teste de Wald. 
McCulloch e Neuhaus (2011a) estudaram o impacto de uma incorreta especificação nas estimativas dos efeitos fixos, nas estimativas das componentes de variância e nas predições dos efeitos aleatórios no MLogM. Eles usaram a distribuição Tukey para a geração dos efeitos aleatórios e estimaram os parâmetros do modelo assumindo distribuição normal e Tukey para os efeitos aleatórios. Nesse trabalho encontraram que as estimativas para o intercepto podem ser viesadas quando a distribuição do intercepto aletório é muito diferente da normal, enquanto para as estimativas dos outros parâmetros foi encontrado pouco viés. Em relação à predição dos efeitos aleatórios, foi observado que o erro médio quadrático de predição foi um pouco maior quando a distribuição assumida foi a normal.

Neuhaus et al. (2011) afirmaram que não estão de acordo com os resultados de Litière et al. (2007). Esses autores analisaram novamente os cenários e encontraram que a incorreta especificação dos efeitos aleatórios conduz apenas a um pequeno incremento do erro tipo II no teste de Wald. Além disso, eles alegam que para demonstrar o impacto da incorreta especificação dos efeitos aleatórios é necessário variar a distribuição assumida, mantendo constante a verdadeira distribuição dos efeitos aleatórios. Recentemente, Litière et al. (2011) apresentaram uma resposta a Neuhaus et al. (2011) na qual defendem o enfoque de variar a verdadeira distribuição dos efeitos aleatórios e estimar os parâmetros assumindo normalidade. Litière et al. (2011) consideraram um MLogM e por meio de simulações encontraram que a potência do teste de Wald é afetada pela incorreta especificação da distribuição dos efeitos aleatórios.

McCulloch e Neuhaus (2011b) estudaram teoricamente e por simulações quão bem os efeitos aleatórios preditos recuperam a verdadeira distribuição dos mesmos, assim como a precisão das predições dos efeitos aleatórios. Eles consideraram dois modelos, MLM e MLogM, nos quais a verdadeira distribuição dos efeitos aleatórios foi normal, exponencial ou Tukey. Nesse estudo foi encontrado que a forma da distribuição dos efeitos aleatórios preditos não necessariamente coincide com a verdadeira distribuição e que, assumir incorretamente a distribuição para os efeitos aleatórios só ocassiona uma modesta degradação no erro quadrado médio da predição. Neuhaus et al. (2012) analisaram o impacto devido à incorreta especificação da distribuição conjunta do intercepto e inclinação aleatórios no viés das estimativas no MLGM. Por meio de resultados analíticos e de simulações, eles mostraram que há pouco viés nas estimativas dos parâmetros.

Como mostrado até agora, existem controvérsias no assunto. Além disso, a maioria dos estudos está centrada no MLogM e não há estudos que considerem outra distribuição para a variável resposta. Por esta razão, teve-se o intuito de analisar o impacto de uma incorreta especificação da distribuição do efeito aleatório nas estimativas dos parâmetros em um modelo multiníveis com variável resposta segundo distribuição Weibull. Na Seção 6.1 apresentam-se o modelo analisado e as suposições associadas e na Seção 6.2 apresentam-se o estudo de 
simulação e os resultados obtidos.

\subsection{Estrutura do modelo}

Na literatura estatística há várias parametrizações para a distribuição Weibull, mas neste trabalho considera-se uma delas, denominada WEI3 e que pode ser encontrada em Stasinopoulos e Rigby (2012). A função de densidade, a média é a variância são dadas por:

$$
\begin{aligned}
f_{Y}(y \mid \mu, \sigma) & =\frac{\sigma}{\kappa}\left(\frac{y}{\kappa}\right)^{\sigma-1} \exp \left[-\left(\frac{y}{\kappa}\right)^{\sigma}\right], \quad \mu>0, \quad \sigma>0, \\
E(Y) & =\mu \\
\mathrm{e} \quad \operatorname{Var}(Y) & =\mu^{2}\left[\Gamma\left(\frac{2}{\sigma}+1\right) \Gamma^{-2}\left(\frac{1}{\sigma}+1\right)-1\right],
\end{aligned}
$$

em que $\kappa=\mu / \Gamma(1 / \sigma+1)$ e $\Gamma(\cdot)$ corresponde à função gama.

Supondo $y_{i j}$ como a $j$-ésima medida do $i$-ésimo grupo, a estrutura matemática do modelo a estudar é a seguinte:

$$
\begin{aligned}
y_{i j} \mid u_{1 i}, u_{2 i} & \stackrel{i n d}{\sim} W E I 3\left(\mu_{i j}, \sigma_{i j}\right), \\
\log \left(\mu_{i j}\right) & =\boldsymbol{X}_{1 i, j} \cdot \boldsymbol{\beta}_{1}+u_{1 i} \\
\text { e } \quad \log \left(\sigma_{i j}\right) & =\boldsymbol{X}_{2 i, j} \cdot \boldsymbol{\beta}_{2}+u_{2 i},
\end{aligned}
$$

em que $i=1,2, \ldots, N$ representa o número de grupos e $j=1,2, \ldots, n_{i}$ representa o número de observações no grupo $i$. No modelo $\boldsymbol{X}_{1 i}$ e $\boldsymbol{X}_{2 i}$ são matrizes de planejamento para o grupo $i$ e conhecidas, enquanto $\boldsymbol{X}_{1 i, j}$. e $\boldsymbol{X}_{2 i, j}$. correspondem às linhas $j$-ésima de $\boldsymbol{X}_{1 i}$ e $\boldsymbol{X}_{2 i}$, respectivamente; $\boldsymbol{\beta}_{1}$ e $\boldsymbol{\beta}_{2}$ são vetores de efeitos fixos desconhecidos. O modelo assume que $u_{1 i} \stackrel{\text { iid }}{\sim} N\left(0, \tau_{1}^{2}\right)$ e $u_{2 i} \stackrel{\text { iid }}{\sim} N\left(0, \tau_{2}^{2}\right)$. Assim, o vetor de parâmetos do modelo está dado por $\boldsymbol{\theta}=\left(\boldsymbol{\beta}_{1}^{T}, \boldsymbol{\beta}_{2}^{T}, \tau_{1}, \tau_{2}\right)^{T}$.

A função de verossimilhança para o $i$-ésimo grupo é dada por

$$
L_{i}(\boldsymbol{\theta})=\iint_{\Re^{2}}\left[\prod_{j=1}^{n_{i}} f\left(y_{i j} \mid \mu_{i j}, \sigma_{i j}\right)\right] p\left(u_{1 i} \mid \tau_{1}\right) p\left(u_{2 i} \mid \tau_{2}\right) d u_{1 i} d u_{2 i},
$$

em que $p(\cdot, a)$ corresponde à função densidade da distrbuição normal com média 0 e variância $a^{2}$. As integrais na expressão (6.2) não têm forma fechada e aproximações são necessarias para a obtenção das estimativas dos parâmetros do modelo (Pinheiro e Chao, 2006). Neste trabalho foi usada a quadratura de Gauss-Hermite, que é explicada com detalhes no Capítulo 7. Para obter as estimativas dos parâmetros $\boldsymbol{\theta}$ do modelo foi usado o método de máxima verossimilhança no qual o objetivo é maximizar a função de $\log$-verossimilhança, $l(\boldsymbol{\theta})$ dada 
por

$$
l(\boldsymbol{\theta})=\sum_{i=1}^{N} \log \left(L_{i}(\boldsymbol{\theta})\right) .
$$

\subsection{Estudo de simulação}

O estudo de simulação Monte Carlo tem como objetivo avaliar o impacto de assumir incorretamente a distribuição dos efeitos aleatórios nas estimativas do modelo (6.4). Neste trabalho foi adotada a abordagem utilizada por Verbeke e Lesaffre (1997), Agresti et al. (2004), Litière et al. (2007, 2008, 2011) e Alonso et al. (2008, 2010), na qual variamos a verdadeira distribuição para a geração dos efeitos aleatórios e se estimaram os parâmetros do modelo foram obtidos assumindo normalidade para os efeitos aleatórios. Foram consideradas seis distribuições para os efeitos aleatórios: normal, uniforme, exponencial, log-gama, $\log$-normal e mistura simétrica de duas normais como definidas em McCulloch e Neuhaus (2011b). Com esta escolha abrange-se uma gama de distribuições que variam desde asimétricas a simétricas. Todas as distribuições foram transformadas para satisfazer as suposições de média 0 e variâncias dadas por $\tau_{1}^{2}$ e $\tau_{2}^{2}$ para $u_{1 i}$ e $u_{2 i}$, respectivamente. A Figura 6.1 apresenta os gráficos das funções densidade para as seis distribuições consideradas.
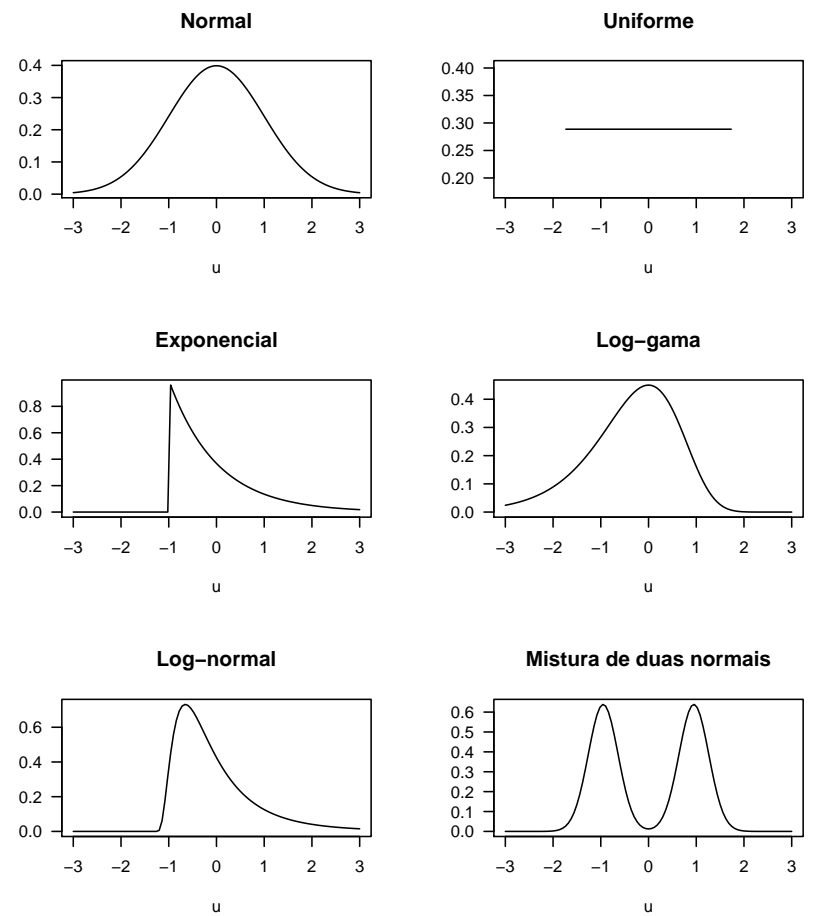

Figura 6.1: Distribuições consideradas para a geração dos efeitos aleatórios, cada uma com média 0 e variância unitária. 
Para a geração das amostras foi usado o seguinte modelo:

$$
\begin{aligned}
y_{i j} \mid u_{1 i}, u_{2 i} & \stackrel{i n d}{\sim} W E I 3\left(\mu_{i j}, \sigma_{i j}\right), \\
\log \left(\mu_{i j}\right) & =\beta_{10}+\beta_{1 b} x_{1 b, i}+\beta_{1 w} x_{1 w, j}+u_{1 i} \\
\text { e } \quad \log \left(\sigma_{i j}\right) & =\beta_{20}+\beta_{2 b} x_{2 b, i}+\beta_{2 w} x_{2 w, j}+u_{2 i},
\end{aligned}
$$

com $i=1,2, \ldots, N$ número de grupos e $j=1,2, \ldots, n_{i}$ observações por grupo. O número de observações por grupo $n_{i}$ foi mantido fixo em 5 e foram usados vários valores para o número de grupos $N=5,10,15,20,25,35,50,65,85,100$. As variáveis $x_{1 b, i}$ e $x_{2 b, i}$ representam variáveis intergrupais enquanto $x_{1 w, j}$ e $x_{2 w, j}$ representam variáveis intragrupais. As variáveis $x_{1 b, i}, x_{1 w, j}, x_{2 b, i}$ e $x_{2 w, j}$ foram geradas segundo uma distribuição $U(0,1)$. O vetor de parâmetros no estudo é dado por $\boldsymbol{\theta}=\left(\beta_{10}, \beta_{1 b}, \beta_{1 w}, \beta_{20}, \beta_{2 b}, \beta_{2 w}, \tau_{1}, \tau_{2}\right)^{T}$. No estudo de simulação os efeitos fixos foram mantidos constantes com os seguintes valores: $\beta_{10}=1, \beta_{1 b}=2, \beta_{1 w}=1$, $\beta_{20}=-1.5, \beta_{2 b}=3, \beta_{2 w}=3.5$. Foram considerados dois valores para a variância dos efeitos aleatórios, $\tau_{1}^{2}=\tau_{2}^{2}=1$ e $\tau_{1}^{2}=\tau_{2}^{2}=1,5$. Para cada combinação de $N$, variância dos efeitos aleatórios e distribuições dos efeitos aleatórios foram geradas 10000 amostras segundo o modelo (6.4). Para cada amostra foi obtido o vetor de estimativas $\hat{\boldsymbol{\theta}}$ do vetor $\boldsymbol{\theta}$ maximizando a função de log-verossimilhança (6.3) usando 21 pontos de quadratura para a aproximação das integrais.

Assim como em Verbeke e Lesaffre (1997), neste trabalho foi usada a distância relativa $\|\hat{\boldsymbol{\theta}}-\boldsymbol{\theta}\| /\|\boldsymbol{\theta}\|$ para avaliar o impacto da incorreta especificação nas estimativas. Quanto menor for o valor dessa distância, menor é o impacto. A Figura 6.2 apresenta a mediana da distância relativa entre $\hat{\boldsymbol{\theta}}$ e $\boldsymbol{\theta}$ para cada combinação de $N$ e distribuição dos efeitos aleatórios com $\tau_{1}=\tau_{2}=1$. Da figura observa-se que a distância relativa diminuiu à medida que o número de grupos $N$ aumentou. No entanto, na figura não há evidências claras de que, assumindo a distribuição normal para os efeitos aleatórios, a distância entre $\hat{\boldsymbol{\theta}}$ e $\boldsymbol{\theta}$ aumentou quando a verdadeira distribuição dos efeitos aleatórios não é normal. Por esta razão, foi calculada a mediana da distância relativa para cada $k$-ésimo componente do vetor de parâmetros para avaliar o impacto da incorreta especificação para cada estimativa e os resultados são mostrados na Figura 6.3. Da figura observa-se que a distância relativa diminuiu à medida que $N$ aumentou para os interceptos, para $\beta_{1 b}$, para $\beta_{2 b}$ e para as componentes de variância. A distância relativa para os coeficientes $\beta_{1 w}$ e $\beta_{2 w}$, associados às covariáveis intragrupais, parece ser constante com distância relativa próxima de 0 para $\beta_{1 w}$ e ao redor de 0,37 para $\beta_{2 w}$. Para $\tau_{1}$ e $\tau_{2}$ observa-se que a distância foi maior quando a verdadeira distribuição dos efeitos aleatórios foi assimétrica (exponencial e log-normal). A diferença das distâncias entre o caso normal e o caso exponencial $/ \log$-normal foi ao redor de $12 \%$ e essa diferença tende a 0 à medida que $N$ aumenta.

A Figura 6.4 apresenta a mediana da distância relativa para cada combinação de $N$ e 
distribuição dos efeitos aleatórios com $\tau_{1}=\tau_{2}=1.5$. Para este caso observa-se o mesmo padrão que o da Figura 6.2, em que a distância relativa diminui à medida que o número de grupos aumenta. Entretanto, as distâncias para $\tau_{1}=\tau_{2}=1.5$ são ligeiramente mais elevadas das observadas no caso de $\tau_{1}=\tau_{2}=1$. Na Figura 6.5 apresentam-se as medianas das distâncias relativas para cada $k$-ésimo componente do vetor de parâmetros e o padrão observado é o mesmo que na Figura 6.3, em que a distância relativa para os coeficientes $\beta_{1 w}$ e $\beta_{2 w}$ foi constante ao redor de 0 e 0,37 , respectivamente e, as distâncias relativas para $\tau_{1} \mathrm{e}$ $\tau_{2}$ maiores quando a distribuição dos efeitos aleatórios é asimétrica.

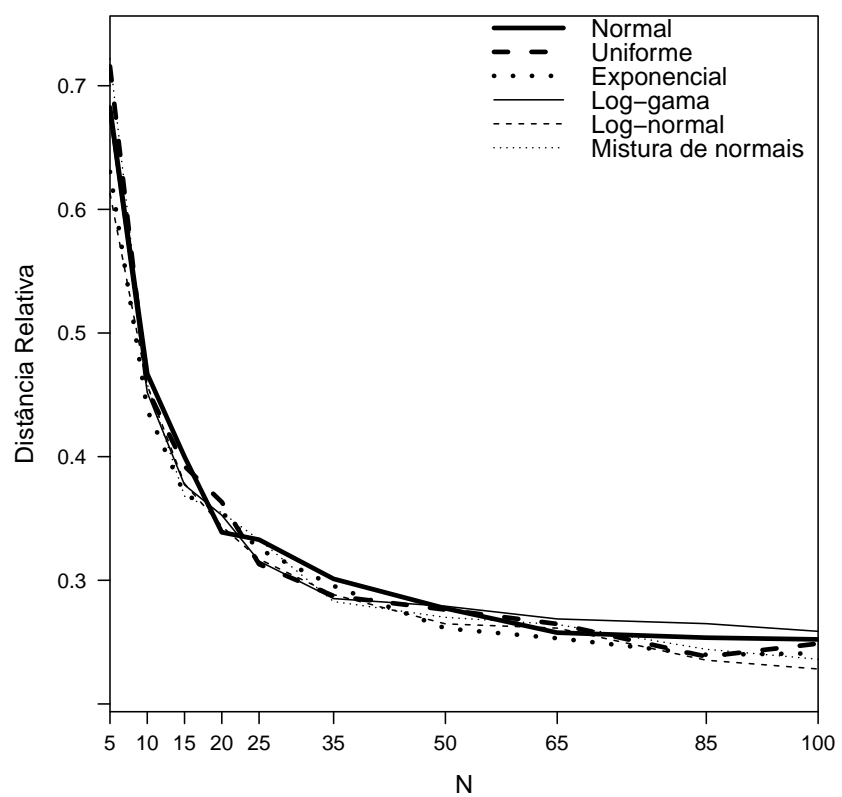

Figura 6.2: Mediana da distância relativa ente $\hat{\boldsymbol{\theta}}$ e $\boldsymbol{\theta}$ para cada uma das distribuiçôes dos efeitos aleatórios com $\tau_{1}=\tau_{2}=1$.

\subsection{Conclusões}

Por meio de um estudo de simulação foi estudado o impacto de assumir incorretamente a distribuição dos efeitos aleatórios em um modelo multiníveis Weibull no qual há interceptos aleatórios nos modelos dos dois parâmetros da distribuição. Neste estudo os efeitos aleatórios foram gerados de seis distribuições diferentes mas os parâmetros do modelo foram estimaos assumindo distribuição normal para os efeitos aleatórios. Nos cenários considerados não foi encontrado impacto sobre as estimativas dos interceptos nem nas estimativas dos coeficientes associados às variáveis intergrupais nem intragrupais. Somente foi encontrado impacto nas estimativas das componentes de variância $\left(\hat{\tau}_{1}\right.$ e $\left.\hat{\tau}_{2}\right)$ quando a verdadeira distribuição dos efeitos aleatórios foi assimétrica, exponencial e $\log$-normal. Os resultados aqui obtidos estão de acordo com as conclusões de Neuhaus et al. (1992), Heagerty e Kurland (2001), McCulloch e Neuhaus (2011a) e McCulloch e Neuhaus (2011b), os quais afirmam que a incorreta 


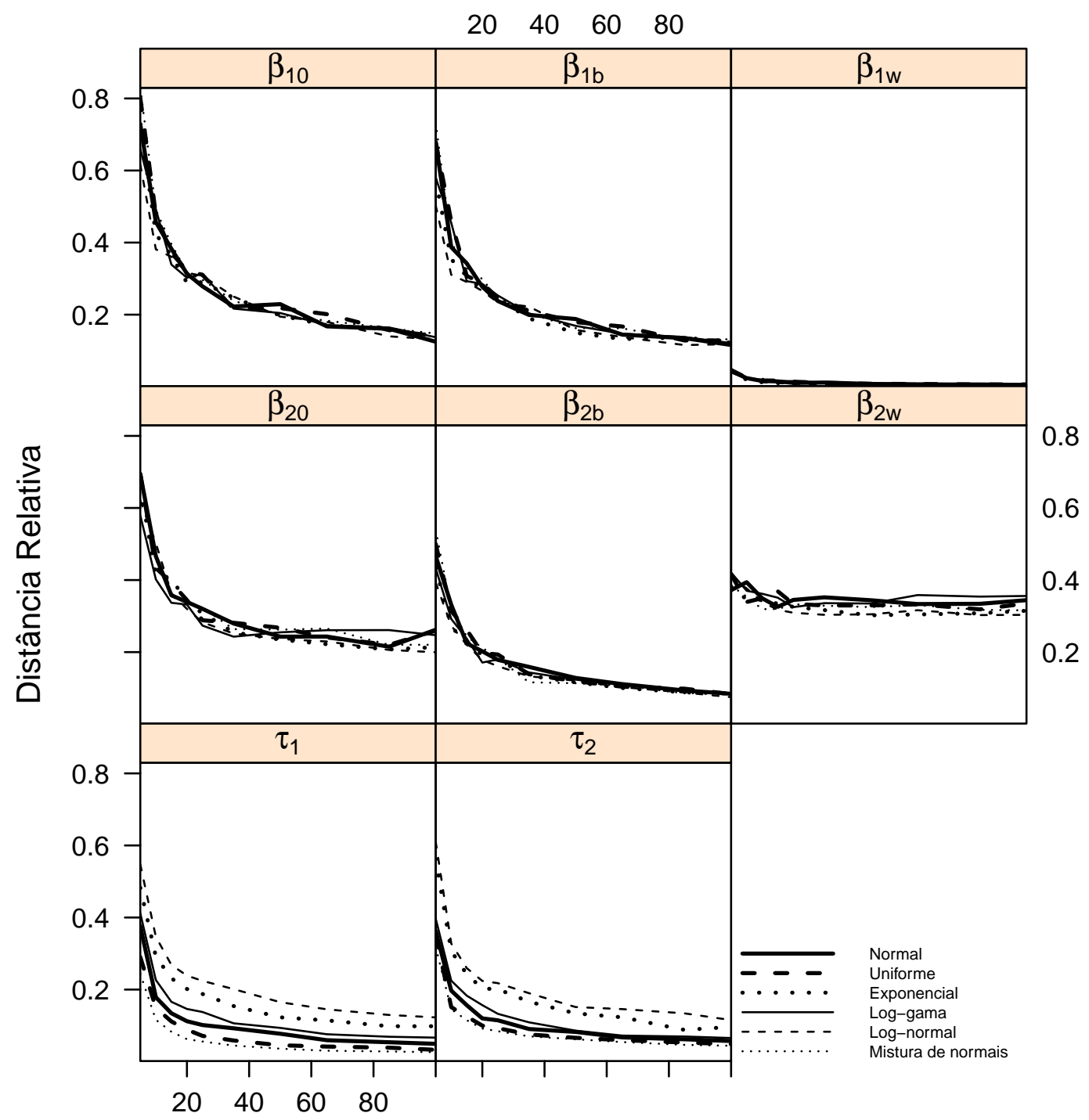

$\mathrm{N}$

Figura 6.3: Mediana da distância relativa entre $\hat{\theta}_{k}$ e $\theta_{k}$ para cada uma das distribuiçôes dos efeitos aleatórios $\operatorname{com} \tau_{1}=\tau_{2}=1$.

especificação da distribuição dos efeitos aleatórios tem pouco impacto nos MLGM. 


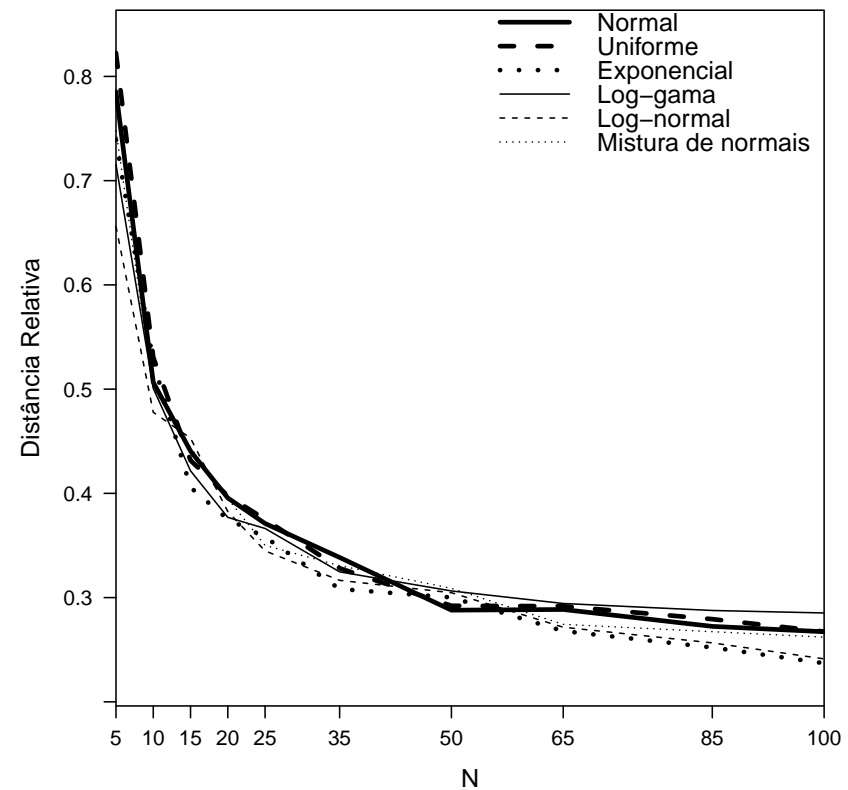

Figura 6.4: Mediana da distância relativa ente $\hat{\boldsymbol{\theta}}$ e $\boldsymbol{\theta}$ para cada uma das distribuiçôes dos efeitos aleatórios com $\tau_{1}=\tau_{2}=1.5$. 


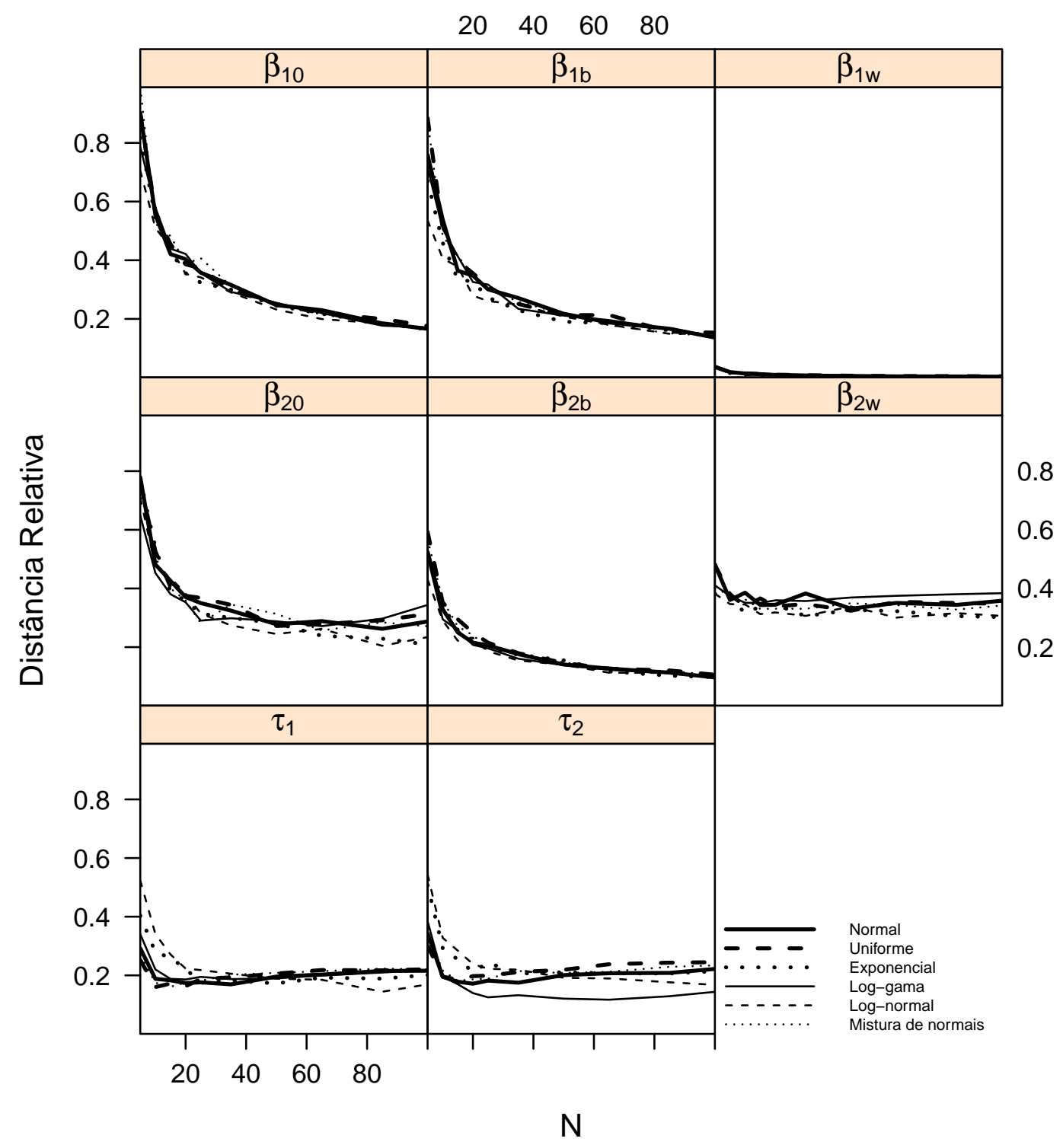

Figura 6.5: Mediana da distância relativa entre $\hat{\theta}_{k}$ e $\theta_{k}$ para cada uma das distribuiçôes dos efeitos aleatórios com $\tau_{1}=\tau_{2}=1.5$. 


\section{Capítulo 7}

\section{Aspectos computacionais}

Neste capítulo apresentam-se vários procedimentos usados no decorrer do presente documento e que serão explicados aqui com mais detalhes. Na Seção 7.1 apresenta-se a quadratura de Gauss-Hermite nas versões usual e adaptativa, úteis para aproximar integrais univariadas ou multivariadas; três exemplos simples ilustram a utilização do método. Na Seção 7.2 é proposta uma nova forma para melhorar a quadratura Gauss-Hermite adaptativa multivariada, que consiste em eliminar aquelas combinações dos pontos de quadratura que têm pesos inferiores a uma cota especial e que garante que os pontos restantes pertencem à região de maior valor do integrando.

\subsection{Quadratura Gauss-Hermite}

A quadratura Gauss-Hermite (QGH) é uma ferramenta para aproximar a integral de uma função $g(x)$ definida na reta real por meio de uma soma ponderada na qual a variável $x$ é substituída por $n$ pontos de quadratura. Cada um dos pontos de quadratura, denotados por $p_{i}$, são avaliados na função e os resultados são ponderados pelos pesos de quadratura denotados por $w_{i}$. O método pode ser resumido por meio da seguinte expressão:

$$
\int_{\Re} g(x) d x \approx \sum_{i=1}^{n} g\left(p_{i}\right) \exp \left(p_{i}^{2}\right) w_{i} .
$$

O conjunto dos pontos de quadratura $\boldsymbol{P}=\left\{p_{1}, p_{2}, \ldots, p_{n}\right\}$ corresponde às raízes do polinómio de Hermite dado por

$$
H_{n}(x)=(-1)^{n} e^{-x^{2}} \frac{d^{n}}{d x^{n}} e^{-x^{2}},
$$

e as componentes do conjunto de pesos $\boldsymbol{W}=\left\{w_{1}, w_{2}, \ldots, w_{n}\right\}$ são obtidas como

$$
w_{i}=\frac{2^{n-1} n ! \sqrt{\pi}}{n^{2}\left[H_{n-1}\left(x_{i}\right)\right]^{2}} .
$$

Na linguagem R, há disponíveis várias funções que fornecem os pontos e pesos de qua- 
dratura, entre elas destacam-se gauss.quad de Smyth et al. (2011), ghq de Broström e Holmberg (2011b) e gaussHermiteData de Blocker (2011). A ordem da quadratura está dada por $n$, o número de pontos de quadratura, e quanto mais próxima $g(x)$ for de $\exp \left(x^{2}\right)$, melhor a aproximação obtida com o método. De Demidenko (2004) podem-se destacar alguns comentarios em relação à QGH. Primeiro, quanto mais afastado de 0 estiver o ponto, menor o peso associado. Segundo, os pontos de quadratura são simétricos ao redor de 0 e pontos com mesmo valor absoluto têm pesos iguais. Terceiro, com um número de pontos $n$ superior a 13 é pouco provável uma melhora na precisão porque os pesos se aproximam de 0 rapidamente quando $n \rightarrow \infty$. Como ilustração, suponha-se que o interesse seja obter os pontos e pesos de quadratura para $n=5$. Assim, é possível usar o comando gauss. quad ( $n=5$, kind="hermite") obtendo-se os vetores de pontos e pesos dados por

$$
\begin{aligned}
\boldsymbol{P} & =\{-2,0202 ;-0,9586 ; 0 ; 0,9586 ; 2,0202\} \\
\text { e } \quad \boldsymbol{W} & =\{0,02 ; 0,3936 ; 0,9453 ; 0,3936 ; 0,02\} .
\end{aligned}
$$

Como exemplo suponha-se que o interesse seja obter numéricamente uma aproximação da integral da função $g_{1}(x)=\exp \left(-(x-1)^{2}\right)$ sobre a reta real usando cinco pontos de quadratura. Na Figura 7.1 apresenta-se o perfil da função $g_{1}(x)$. No eixo horizontal foram posicionados os pontos de quadratura $p_{i}$ representados por pontos pretos. Aproximando a integral de $g_{1}(x)$ por meio da expressão (7.1), e usando os pontos $\boldsymbol{P}$ e os pesos $\boldsymbol{W}$ anteriormente calculados, o resultado obtido é 1,77134, enquanto o valor exato da integral é 1,77245. Observa-se então que neste exemplo a aproximação gera um valor próximo do verdadeiro valor da integral.

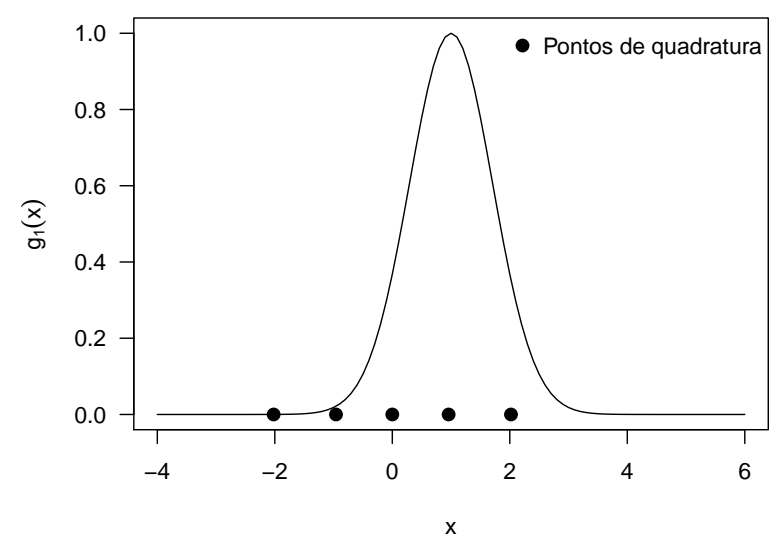

Figura 7.1: Função $g_{1}(x)$ e pontos de quadratura.

Suponha-se agora que o interesse é obter numéricamente uma aproximação da integral da função $g_{2}(x)=\exp \left(-5(x-3)^{2}\right)$ na reta usando os mesmos cinco pontos de quadratura do exemplo anterior. No gráfico da esquerda na Figura 7.2 apresenta-se o perfil da função 
$g_{2}(x)$ e os pontos de quadratura $p_{i}$. Ao usar a expressão (7.1), usando os pontos e pesos de quadratura $\boldsymbol{P}$ e $\boldsymbol{W}$, obtem-se que a aproximação da integral da função $g_{2}(x)$ é 0,00972, enquanto o valor correto para integral é de 0,79266. Esta aproximação incorreta deve-se a que os pontos de quadratura estão afastados da região em que a função $g_{2}(x)$ tem valores mais altos, por tanto, isso ocasiona que a aproximação com a expressão (7.1) seja insatisfatória. Uma solução simples é incrementar o número de pontos de quadratura $n$ para tentar cobrir a região em que a função tem valores mais altos. No entanto, essa solução não assegura uma boa aproximação porque os pontos de quadratura afastados de zero têm pesos baixos na expressão (7.1) o qual ocasiona que esses pontos, localizados na parte de maiores valores da função, não contribuam muito para a aproximação devido a sua baixa importância. Além disso, em algumas situações, aumentar de forma exagerada $n$ pode acarretar custos e problemas computacionais. Para contornar esse problema, existe uma versão do método chamada quadratura Gauss-Hermite adaptativa (Liu e Pierce, 1994; Pinheiro e Bates, 1995) a qual transforma os pontos $p_{i}$, centrando-os e espalhando-os ao redor do ponto de máximo $\hat{x}$ da função $\log (g(x))$. A transformação dos pontos de quadratura $p_{i}$ nos novos pontos $p_{i}^{\star}$ é dada pela expressão $p_{i}^{\star}=\sqrt{2} \hat{\sigma} p_{i}+\hat{x}$ em que

$$
\hat{\sigma}^{2}=\left[-\left.\frac{d^{2}}{d x^{2}} \log (g(x))\right|_{x=\hat{x}}\right]^{-1} .
$$

Assim a aproximação da integral da função $g(x)$ na reta real pela quadratura Gauss-Hermite adaptativa (QGHA) é dada por

$$
\int_{\Re} g(x) d x \approx \sqrt{2} \hat{\sigma} \sum_{i=1}^{n} g\left(p_{i}^{\star}\right) \exp \left(p_{i}^{2}\right) w_{i} .
$$
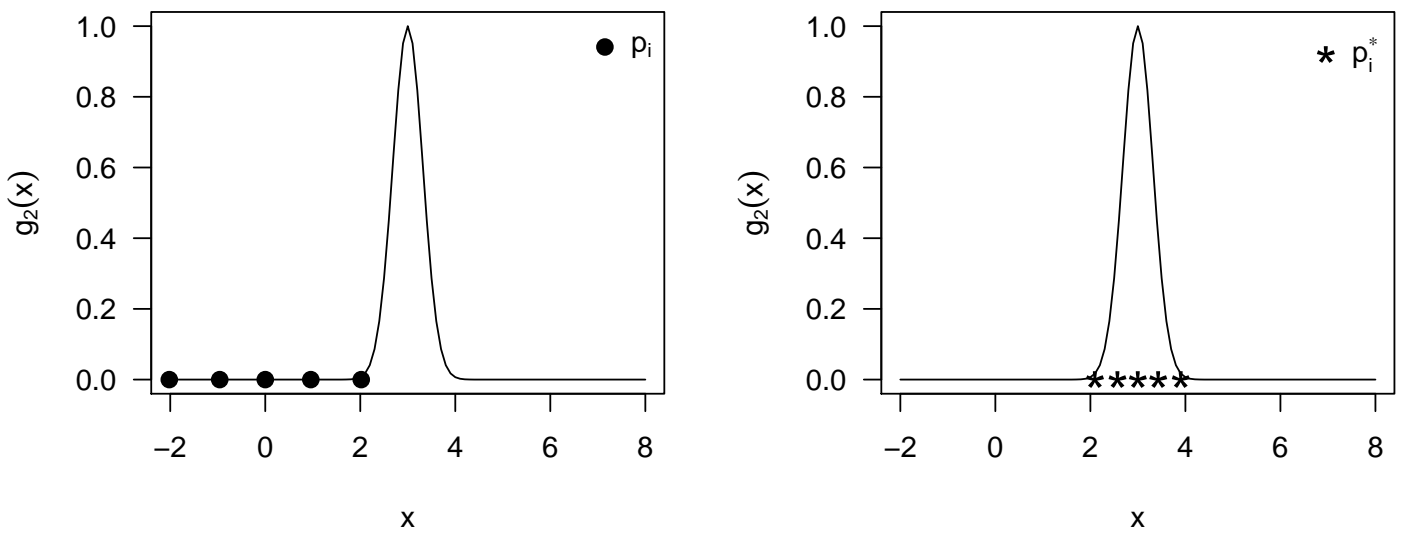

Figura 7.2: Função $g_{2}(x)$ com os pontos de quadratura $p_{i}$ e $p_{i}^{\star}$.

Note-se que na expressão (7.2) a função $g(x)$ é avaliada nos pontos $p_{i}^{\star}$ enquanto o fator 
$\exp (\cdot)$ é avaliado nos pontos $p_{i}$. Para o exemplo com a função $g_{2}(x)$ tem-se que $\hat{x}=3$ e $\hat{\sigma}=$ 0.31623. Assim, os novos pontos de quadratura são $\boldsymbol{P}^{\star}=\{2.0965,2.5713,3,3.4287,3.9035\}$. No painel direito da Figura 7.2 apresenta-se a função $g_{2}(x)$ com os novos pontos de quadratura $p_{i}^{\star}$ e observa-se como os cinco novos pontos estão centrados e distribuidos na região de maiores valores de $g_{2}(x)$. Aplicando a expressão (7.2), com os novos pontos de quadratura, obtem-se que a integral é 0,79266, que é uma aproximação melhor da integral de $g_{2}(x)$.

A fim de facilitar a compreensão dos métodos QGH e QGHA no caso multidimensional, um exemplo no caso bidimensional é apresentado em detalhe. Suponha-se que o interesse é calcular a integral de $g\left(x_{1}, x_{2}\right)$ em $\Re^{2}$ usando cinco pontos de quadratura. Suponha-se também que a função $g\left(x_{1}, x_{2}\right)$ corresponde à função densidade de uma distribuição normal bivariada com média $\boldsymbol{\mu}=(-2,-2)^{T}$ e variâncias e covariânza dadas por 1,2 e -0.5 , respectivamente. Neste caso é preciso construir um conjunto com elementos bidimensionais, baseado nos pontos de quadratura $\boldsymbol{P}=\left\{p_{1}, p_{2}, \ldots, p_{5}\right\}$, os quais correspondem ao produto cartesiano de $\boldsymbol{P}$. Denotando por $\boldsymbol{Z}$ esse conjunto com 25 elementos tem-se que $\boldsymbol{Z}$ está formado por

$$
\begin{gathered}
\boldsymbol{Z}=\left\{\left(p_{1}, p_{1}\right),\left(p_{1}, p_{2}\right), \ldots,\left(p_{1}, p_{5}\right),\right. \\
\left(p_{2}, p_{1}\right),\left(p_{2}, p_{2}\right), \ldots,\left(p_{2}, p_{5}\right), \\
\vdots \\
\left.\left(p_{5}, p_{1}\right),\left(p_{5}, p_{2}\right), \ldots,\left(p_{5}, p_{5}\right)\right\} .
\end{gathered}
$$

Os pesos de quadratura, denotados por $\boldsymbol{A}$, são dados pelo produto dos pesos associados a cada um dos elementos de $\boldsymbol{Z}$ e são dados por

$$
\begin{gathered}
\boldsymbol{A}=\left\{w_{1} w_{1}, w_{1} w_{2}, \ldots, w_{1} w_{5},\right. \\
w_{2} w_{1}, w_{2} w_{2}, \ldots, w_{2} w_{5}, \\
\vdots \\
\left.w_{5} w_{1}, w_{5} w_{2}, \ldots, w_{5} w_{5}\right\} .
\end{gathered}
$$

Assim, as expressões para aproximar a integral de $g\left(x_{1}, x_{2}\right)$ em $\Re^{2}$ por meio dos métodos QGH e QGHA são dadas por

$$
\begin{array}{ll}
\text { Para QGH: } & \int_{\Re^{2}} g\left(x_{1}, x_{2}\right) d x_{1} d x_{2} \approx \sum_{i=1}^{25} g\left(\boldsymbol{z}_{i}\right) \exp \left(\boldsymbol{z}_{i}^{T} \boldsymbol{z}_{i}\right) a_{i}, \\
\text { Para QGHA: } & \int_{\Re^{2}} g\left(x_{1}, x_{2}\right) d x_{1} d x_{2} \approx 2|\hat{\boldsymbol{Q}}|^{1 / 2} \sum_{i=1}^{25} g\left(\boldsymbol{z}_{i}^{\star}\right) \exp \left(\boldsymbol{z}_{i}^{T} \boldsymbol{z}_{i}\right) a_{i},
\end{array}
$$

em que $\boldsymbol{z}_{i}$ e $a_{i}$ correspondem a cada um dos elementos de $\boldsymbol{Z}$ e $\boldsymbol{A}$, respectivamente. Os novos pontos de quadratura $\boldsymbol{z}_{i}^{\star}$ para QGHA são centrados ao redor do ponto de máximo 
$\hat{\boldsymbol{x}}$ de $\log \left(g\left(x_{1}, x_{2}\right)\right)$ e são dados por $\boldsymbol{z}_{i}^{\star}=\hat{\boldsymbol{x}}+\sqrt{2} \hat{\boldsymbol{Q}}^{1 / 2} \boldsymbol{z}_{i}$, em que $\hat{\boldsymbol{Q}}^{1 / 2}$ corresponde à raíz quadrada de Cholesky da matriz de curvatura $\hat{\boldsymbol{Q}}$ dada por

$$
\hat{\boldsymbol{Q}}=\left[-\left.\frac{d^{2}}{d x_{1} d x_{2}} \log \left(g\left(x_{1}, x_{2}\right)\right)\right|_{\boldsymbol{x}=\hat{\boldsymbol{x}}}\right]^{-1} \text {. }
$$

A Figura 7.3 apresenta as curvas de nível para $g\left(x_{1}, x_{2}\right)$. No gráfico à esquerda foram situados os 25 pontos de quadratura e no gráfico à direita os novos pontos de quadratura $\boldsymbol{z}_{i}^{\star}$ transformados. Ao contrário dos pontos $\boldsymbol{z}_{i}$, observa-se que os pontos $\boldsymbol{z}_{i}^{\star}$ estão melhor localizados na região de maiores valores de $g\left(x_{1}, x_{2}\right)$ e isto é refletido na aproximação da integral. Usando as expressões (7.3) e (7.4) obtém-se que a integral de $g\left(x_{1}, x_{2}\right)$ é 0,4719 com QGH e 0,9999 com QGHA.
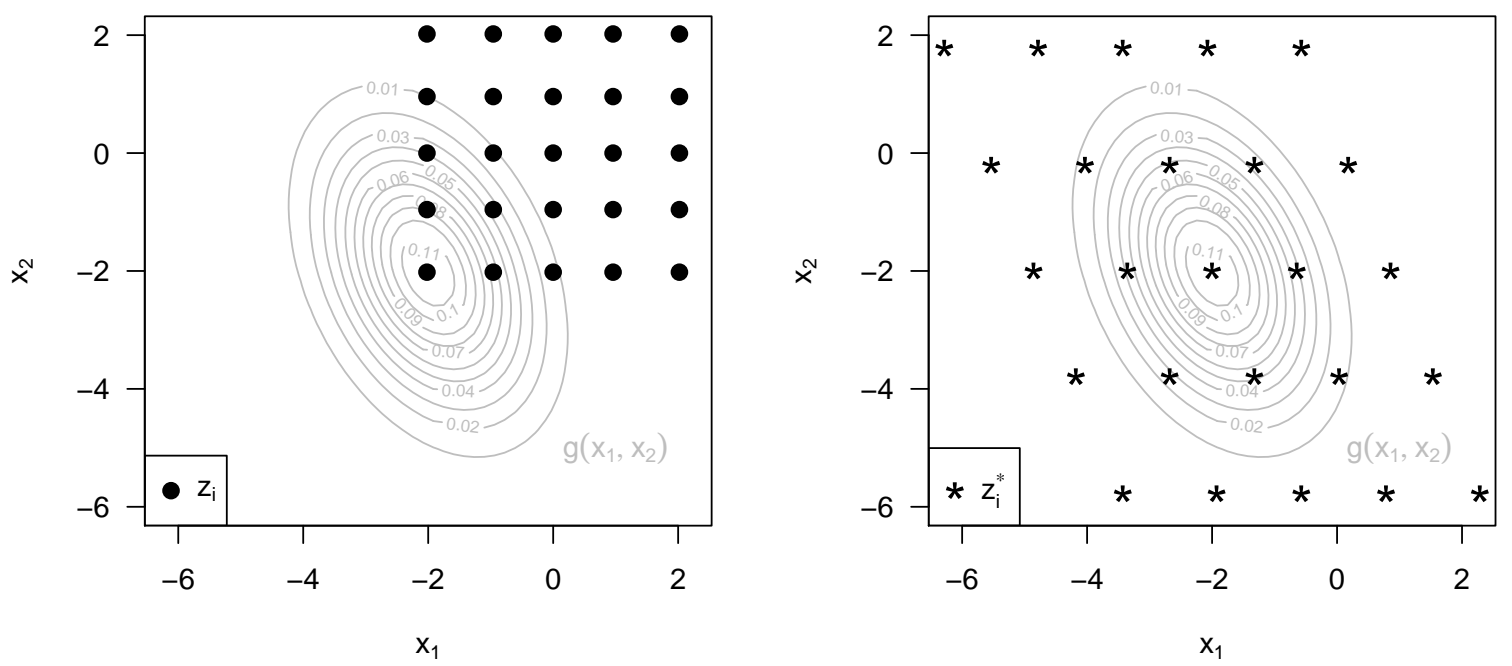

Figura 7.3: Curvas de nível para a função $g\left(x_{1}, x_{2}\right)$ com os pontos de quadratura $\boldsymbol{z}_{i}$ e $\boldsymbol{z}_{i}^{\star}$.

Baseados nas expressões apresentadas para o caso bivariado, os métodos QGH e QGHA podem ser estendidos para o caso de uma integral $q$-dimensional de uma função $g(\boldsymbol{x}) \mathrm{em}$ $\Re^{q}$. Neste caso, com $n$ pontos de quadratura, $Z$ é obtido por meio do produto cartesiano de $\boldsymbol{P}$ e os pesos de quadratura $\boldsymbol{A}$ similarmente são obtidos por meio do produto Kronecker (denotado por $\otimes$ ) dos pesos originais $\boldsymbol{W}$. As expressões para a obtenção de $\boldsymbol{Z}$ e $\boldsymbol{A}$ são as seguintes:

$$
\boldsymbol{Z}=\underbrace{\boldsymbol{P} \times \ldots \times \boldsymbol{P}}_{q \text { vezes }}=\boldsymbol{P}^{q} \quad \text { e } \quad \boldsymbol{A}=\underbrace{\boldsymbol{W} \otimes \ldots \otimes \boldsymbol{W}}_{q \text { vezes }}
$$

Assim, as expressões para aproximar a integral de $g(\boldsymbol{x})$ em $\Re^{q}$ por meio dos métodos QGH 
e QGHA são dadas por

$$
\begin{array}{ll}
\text { Para QGH: } & \int_{\Re^{q}} g(\boldsymbol{x}) d \boldsymbol{x} \approx \sum_{i=1}^{n^{q}} g\left(\boldsymbol{z}_{i}\right) \exp \left(\boldsymbol{z}_{i}^{T} \boldsymbol{z}_{i}\right) a_{i}, \\
\text { Para QGHA: } & \int_{\Re^{q}} g(\boldsymbol{x}) d \boldsymbol{x} \approx|\hat{\boldsymbol{Q}}|^{1 / 2} 2^{q / 2} \sum_{i=1}^{n^{q}} g\left(\boldsymbol{z}_{i}^{\star}\right) \exp \left(\boldsymbol{z}_{i}^{T} \boldsymbol{z}_{i}\right) a_{i},
\end{array}
$$

em que $\boldsymbol{z}_{i}$ e $a_{i}$ correspondem a cada um dos elementos de $\boldsymbol{Z}$ e $\boldsymbol{A}$, respectivamente. Os novos pontos de quadratura $\boldsymbol{z}_{i}^{\star}$ para QGHA são centrados ao redor do máximo $\hat{\boldsymbol{x}}$ de $\log (g(\boldsymbol{x}))$ e são dados por $\boldsymbol{z}_{i}^{\star}=\hat{\boldsymbol{x}}+\sqrt{2} \hat{\boldsymbol{Q}}^{1 / 2} \boldsymbol{z}_{i}$, em que $\hat{\boldsymbol{Q}}^{1 / 2}$ corresponde à decomposição de Cholesky da matriz de curvatura $\hat{\boldsymbol{Q}}$ dada por:

$$
\hat{\boldsymbol{Q}}=\left[-\left.\frac{d^{2}}{d \boldsymbol{x}^{2}} \log (g(\boldsymbol{x}))\right|_{\boldsymbol{x}=\hat{\boldsymbol{x}}}\right]^{-1} .
$$

Dos exemplos anteriores é evidente que a quadratura Gauss-Hermite adaptativa gera melhores resultados que a QGH. No entanto, o custo computacional da aproximação incrementase pela otimização requerida para a transformação dos pontos de quadratura. Se o interesse é resolver uma integral uma vez só, este custo computacional adicional não é expressivo, mas quando a QGHA é usada em um processo iterativo para estimar os parâmetros de um modelo misto, o método torna-se computacionalmente intensivo. Se o tempo de processamento é um aspecto fundamental na obtenção das estimativas dos parâmetros nos modelos mistos, Hartzel et al. (2001) recomendam usar a QGH com poucos pontos de quadratura para obter valores iniciais dos parâmetros e em seguida, usar a QGHA para melhorar a precisão dos resultados. Para mais detalhes da aplicação do método QGHA em modelos mistos, vide Hartzel et al. (2001) e para aspectos técnicos da implementação dos métodos QGH e QGHA recomenda-se Ribeiro et al. (2012).

\subsection{Melhorando a QGHA com a eliminação de pontos}

Um aspecto fundamental na aplicação da QGH e QGHA é a escolha do número $n$ de pontos de quadratura. Um número elevado de pontos de quadratura implica um custo computacional maior e possíveis problemas computacionais. Por exemplo, para um problema que precise resolver uma integral de ordem $q$, o custo computacional aumenta considerávelmente devido a que é necessario considerar todas as combinações dos $n$ pontos para cada uma das integrais, gerando isto um conjunto de $n^{q}$ pontos. Por exemplo, para obter a integral de uma função com três variáveis usando 15 pontos de quadratura, é preciso construir uma matriz com $15^{3}=3375$ combinações de pontos. Para cada uma dessas $n^{q}$ combinações, os novos pesos são obtidos como o produto dos pesos individuais iniciais. No entanto, há algumas dessas $n^{q}$ combinações que têm pesos pequenos e que não contribuem para a aproximação da integral. Por tanto, é preciso contar com uma ferramenta para identificar e eliminar 
essas possíveis combinações que não contribuem e que encarecem o processo de aproximação.

Visando este fim, nesta seção é apresentado o processo de eliminação de pontos de quadratura para a aproximação de uma integral multidimensional apresentado por Hernández et al. (2013). A idéia é a seguinte. Aqueles pontos do novo conjunto de pontos $\boldsymbol{Z}$, tais que os pesos associados $a_{i}$ sejam inferiores a um valor de referência $\psi$, serão removidos e não são usados na aproximação devido a que a importância $a_{i}$ não é expressiva e não contribuem à aproximação da integral. O valor de referência $\psi$ depende de $n, q$ e os pesos por meio da expressão

$$
\psi=\frac{w_{[1]} w_{\left[\frac{n+1}{2}\right]}}{n^{q-1}},
$$

em que $w_{[1]}$ e $w_{\left[\frac{n+1}{2}\right]}$ correspondem, respectivamente, ao mínimo e á mediana dos pesos originais $\boldsymbol{W}$. Usando as expressões (7.6) ou (7.7) com os pontos restantes de $\boldsymbol{Z}$, a soma terá menos elementos, acarretamdo um menor tempo de processamento. No caso de uma única integral essa redução de tempo é irrelevante, mas em um processo iterativo pesado, a diferença pode ser importante.

A função GHQ ( $n$, ndim, pruning=TRUE) apresentada no Capítulo 8 pode ser usada para obter os pontos e pesos de quadratura com o enfoque de eliminação para um número de pontos de quadratura $n$ e dimensão da integral ndim dados. Adicionalmente, na função proposta é possível escolher remover ou não aqueles pontos cujos pesos sejam inferiores a $\theta$ por meio do parâmetro pruning, o valor padrão para esse parâmetro é TRUE. A função GHQ fornece uma lista com três elementos, o primeiro deles corresponde aos pontos de quadratura $\boldsymbol{Z}$, o segundo aos pesos individuais e o terceiro aos pesos $\boldsymbol{A}$ usados na aproximação.

Como ilustração da redução no número de pontos com o enfoque proposto, a Tabela 7.1 apresenta o número de pontos totais com e sem a eliminação de pontos para a aproximação de uma integral com $q=2,3$ considerando $n=3,7,15$ pontos iniciais de quadratura usando a função GHQ. Da tabela observa-se que existe uma diminuição do número de combinações totais com a eliminação dos pontos com pesos inferiores a $\psi$. Na Figura 7.4 apresentam-se os pontos sem e com eliminação para os casos de $q=2$ e $q=3$. Observa-se da figura que as combinações eliminadas estão situadas nas fronteras das regiões e que são mantidas as combinações localizadas na parte central. A vantagem de realizar a eliminação de pontos ao usar uma aproximação pela quadratura de Gauss-Hermite é a diminuição do número de vezes que a função de interesse é avaliada. Isto é particulamente crucial nos modelos mistos no quais é preciso calcular várias integrais em um processo iterativo para a obtenção das estimativas dos parâmetros do modelo. 
Tabela 7.1: Número de combinaçôes de pontos finais para solucionar $q=2,3$ integrais com $n=$ $3,7,15$ pontos de quadratura.

\begin{tabular}{c|c|c|c|c|c|c}
\hline & \multicolumn{3}{|c|}{$q=2$} & \multicolumn{3}{c}{$q=3$} \\
\hline$n$ & Sem corte & Com corte & Pd(\%) & Sem corte & Com corte & Pd(\%) \\
\hline 3 & 9 & 5 & 44,44 & 27 & 19 & 29,63 \\
7 & 49 & 37 & 24,49 & 343 & 251 & 26,82 \\
15 & 225 & 185 & 17,77 & 3375 & 2199 & 34,84 \\
\hline
\end{tabular}

Pd significa percentagem de diminuição.

Sem corte, $n=15$ e $q=2$

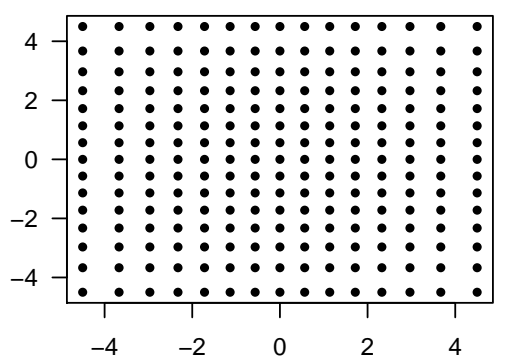

Sem corte, $n=15$ e $q=3$



Com corte, $n=15$ e $q=2$

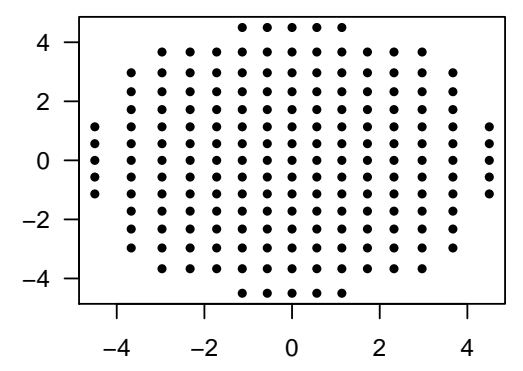

Com corte, $n=15$ e $q=3$

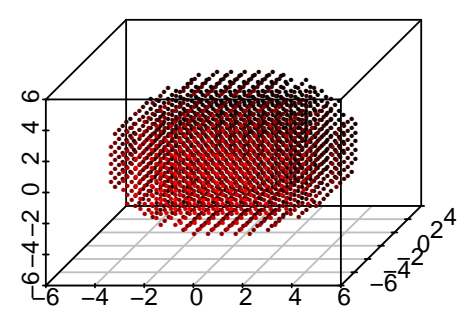

Figura 7.4: Combinações dos pontos de quadratura sem e com a eliminação. 


\section{Capítulo 8}

\section{Pacote 'wlmm'}

Neste capítulo apresentam-se as principais funções do pacote 'wlmm' desenvolvido na linguagem $\mathrm{R}$ com o intuito de ajustar modelos multiníveis Weibull. A função principal é wlmmML, que permite obter as estimativas dos parâmetros do modelo pelo método de máxima verossimilhança. Esta função é bastante flexível é permite considerar intercepto e inclinação aleatórias nos modelos dos dois parâmetros da distribuição Weibull com qualquer uma das parametrizações $W E I, W E I 2$ e $W E I 3$ descritas no Apêndice A. A maximização da função de verossimilhança pode ser realizada com o otimizador optim ou nlminb. A aproximação das integrais envolvidas na obtenção da função densidade marginal do vetor de observações para cada grupo pode ser realizada com os métodos QGH ou QGHA, como descritos no Capítulo 7. A distribuição dos interceptos aleatórios é flexibilizada e pode-se escolher entre normal, log-gama, logística e Cauchy; por enquanto, só é possível assumir distribuição normal multivariada no caso de interceptos e inclinações aleatórias.

wlmmML Ajuste do modelo multinível Weibull por máxima verossimilhança

\section{Descrição}

Esta função permite obter as estimativas dos parâmetros de um modelo multinível Weibull pelo método de máxima verossimilhança.

\section{Uso}

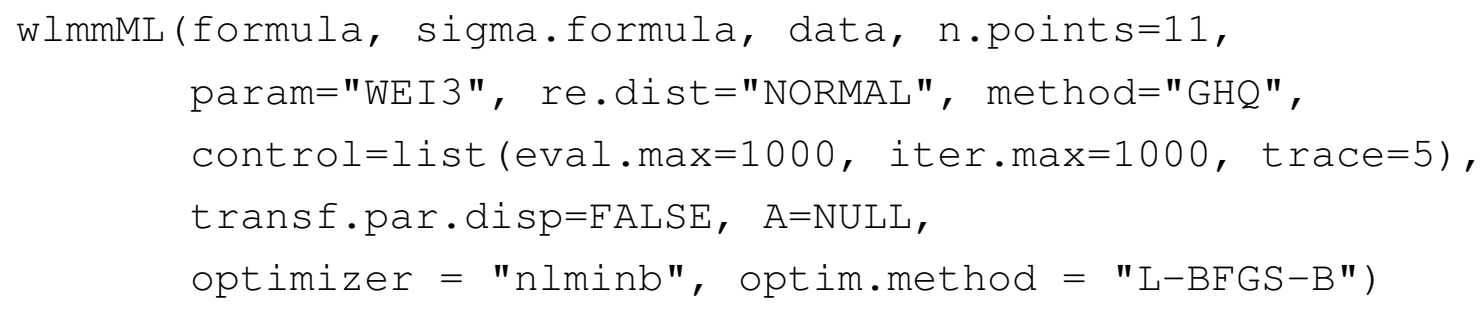

\section{Argumentos}


formula fórmula de duas partes para o preditor linear do parâmetro $\mu$. É usada a mesma estrutura de Bates et al. (2012). No lado esquerdo de $\sim$ é indicada a variável resposta. Na parte direita tem-se as variáveis associadas aos efeitos fixos e entre parênteses as variáveis com coeficientes aleatórios seguidas do símbolo | e a variável que gera os grupos. Por exemplo com resp $\mathrm{x} 1+\mathrm{x} 2+(1+\mathrm{x} 2 \mid \mathrm{grupo})$ temos três coeficientes de efeitos fixos, $\beta_{0}$, $\beta_{x 1}$ e $\beta_{x 2}$, e doís coeficientes com aleatórios devido à variável grupo.

sigma.formula fórmula para o preditor linear do parâmetro $\sigma$. Esta fórmula só deve ter o lado direito com a mesma estrutura do argumento anterior.

data

objeto onde estão armazenadas as variáveis da análise e deve ter a estrutura de um data.frame.

n.points número de pontos de quadratura usados para aproximar cada uma das integrais.

param

tipo de parametrização. Estão disponíveis WEI, WEI2 e WEI3 como no pacote gamlss de Rigby e Stasinopoulos (2005).

re.dist

distribuição para os interceptos aleatórios. Estão disponíveis NORMAL, LG para a distribuição log-gama, LOGISTIC e CAUCHY. Para o caso de interceptos e inclinações aleatórias só é possivel usar a distribuição normal bivariada como distribuição dos efeitos aleatórios.

method

tipo de quadratura Gauss-Hermite para aproximar as integrais. Estão disponíveis GHQ para o caso de interceptos e inclinações aleatórias e a versão adaptativa AGHQ só para o caso de interceptos aleatórios.

control lista com elementos de control e compatíveis com o otimizador nlminb usado para a maximização da função verossimilhança marginal.

transf.par.disp variável lógica, TRUE se os parâmetros associados às distribuições dos efeitos aleatórios devem ser transformados à escala $\Re$ para a busca ou FALSE caso contrario.

$\mathrm{A}$ mínimo valor admisível para a busca dos parâmetros $\tau_{1}$ e $\tau_{2}$ no modelo com interceptos aleatórios.

optimizer otimizador a usar. Estão disponíveis nlminb e optim.

optim.method tipo de método a usar caso o otimizador fornecido seja optim, para mais detalhes veja-se optim. 


\section{Detalhes}

A variável resposta no argumento formula pode ingresar com a informação da censura tipo I à direita na forma usual do pacote survival de Therneau (2012); se a variável status contém a informação da censura para as respostas resp, então a parte esquerda da fórmula é escrita como Surv(resp, status).

\section{Valores}

É obtido um objeto da classe weibullreg.

\section{Exemplos}

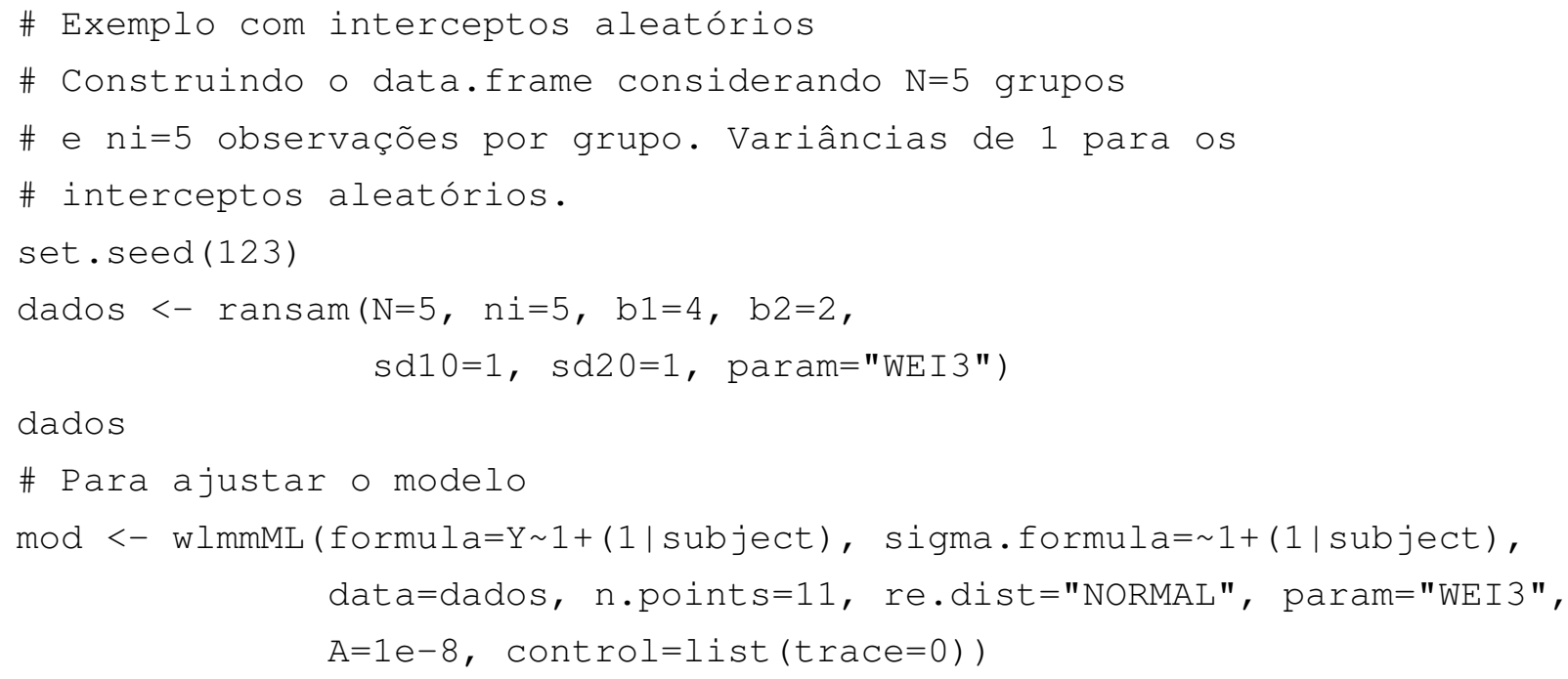

summary $(\bmod )$

\# Exemplo com interceptos e inclinações aleatórios em mu

\# e intercepto aleatório em sigma, com N=5 e ni=5

set. seed (12345)

dados <- ransam $(\mathrm{N}=5, \mathrm{ni}=5, \mathrm{~b} 1=\mathrm{c}(1,2), \mathrm{b} 2=\mathrm{c}(1,1)$,

dados

\# Para ajustar o modelo na escala transformada

mod <- wlmmML (formula=Y 1+x11+(1+x11| subject), 


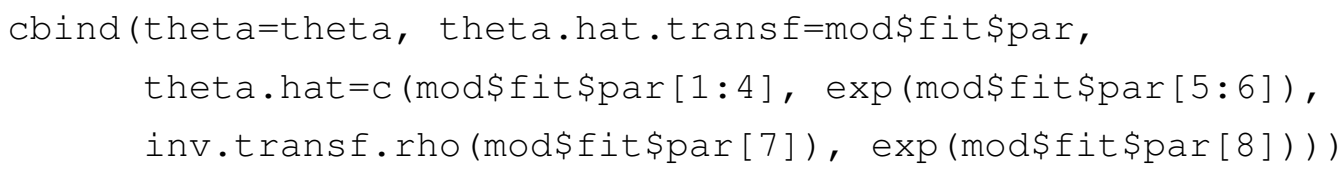

wlmmBUGS Ajuste do modelo multinível Weibull usando OpenBUGS

\section{Descrição}

Esta função permite obter as estimativas do modelo multinível Weibull com interceptos aleatórios normais usando WinBUGS, BRugs, ou OpenBUGS. As distribuições a priori usadas para os efeitos fixos foram $\beta_{i} \sim N(0 ; 0,001)$ e para as variâncias dos interceptos aleatórios foram $\tau_{i} \sim \operatorname{Gama}(0,001 ; 0,001)$ com as parametrizações usadas em BUGS.

\section{Uso}

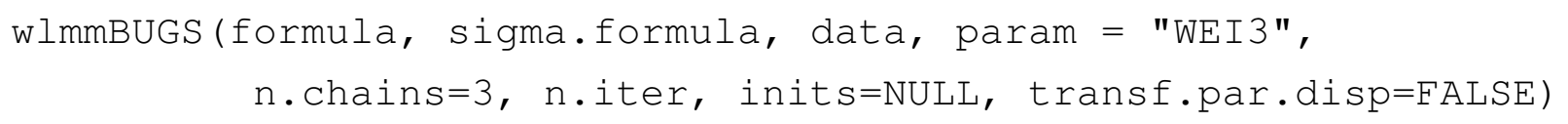

\section{Argumentos}

formula fórmula de duas partes para o preditor linear do parâmetro $\mu$. É usada a mesma estrutura de Bates et al. (2012). Na parte esquerda de $\sim$ é especificada a variável resposta. Na parte direita tem-se as variáveis associadas aos efeitos fixos e entre parênteses as variáveis com coeficientes aleatórios seguidas do símbolo | e a variável que gera os grupos. Por enquanto só é possível incluir interceptos aleatórios e observações sem censura.

sigma.formula fórmula para o preditor linear do parâmetro $\sigma$. Esta fórmula só deve ter parte direita com a mesma estrutura do argumento anterior.

data objeto onde estão armazenadas as variáveis da análise e deve ter a estrutura data.frame.

param tipo de parametrização, estão disponíveis WEI, WEI2 e WEI3 como no pacote gamlss de Rigby e Stasinopoulos (2005).

n.chains número de cadeias a usar.

n.iter número de iterações a usar.

inits lista com os valores inicias para cada uma das cadeias.

transf.par.disp varíavel lógica, TRUE indica que os erros padrão dos interceptos aleatórios devem ser obtidos na escala log; FALSE para a escala usual. 


\section{Valores}

É obtida uma lista da clase mcmc.

\section{Exemplos}



summary (mod)

Referências

Spiegelhalter et al. (2012) e Spiegelhalter et al. (2003).

ransam Gerador de amostras aleatórias

\section{Descrição}

Função usada para gerar amostras aleatórias com distribuição Weibull e efeitos aleatórios.

Uso

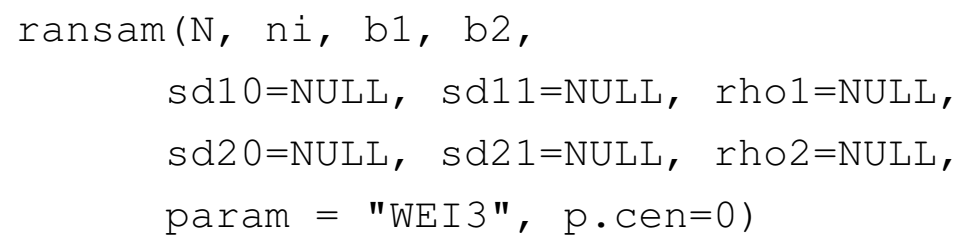

\section{Argumentos}

$\mathrm{N}$

ni número de grupos.

número de observações por grupo. Só e possível igual número de observações por grupo. 
b1

vetor com os efeitos fixos no parâmetro $\mu$.

b2 vetor com os efeitos fixos no parâmetro $\sigma$.

$\operatorname{sd} 10$ erro padrão para o intercepto aleatório no preditor linear em $\mu$.

$\operatorname{sd} 11$ erro padrão para a inclinação aleatória no preditor linear em $\mu$.

rho1 coeficiente de correlação entre o intercepto e inclinação aleatória no preditor linear em $\mu$.

$\operatorname{sd} 20$ erro padrão para o intercepto aleatório no preditor linear em $\sigma$.

$\operatorname{sd} 21$ erro padrão para a inclinação aleatória no preditor linear em $\sigma$.

rho2 coeficiente de correlação entre o intercepto e inclinação aleatória no preditor linear em $\sigma$.

param tipo de parametrização para a geração das observações, estão disponíveis WEI, WEI2 e WEI3 como no pacote gamlss de Rigby e Stasinopoulos $(2005)$.

p.cen percentagem de observações censuradas na amostra. $\mathrm{O}$ valor de percentagem deve estar entre 0 e 1.

\section{Detalhes}

Para obter as observações censuradas calcula-se o quantil p. cen das observações geradas e aquelas maiores ou iguais ao quantil são substituídas pelo valor do quantil.

\section{Valores}

Obtem-se um objeto data. frame com os seguintes elementos:

Y

$\mathrm{x} 1 \mathrm{i}$

$\mathrm{x} 2 \mathrm{i}$

delta

subject variável resposta.

coluna $i$-ésima da matriz associada ao preditor linear em $\mu$.

coluna $i$-ésima da matriz associada ao preditor linear em $\sigma$.

variável indicadora da censura, 0 para censura e 1 para as observações não censuradas.

variável numérica indicando cada um dos grupos.

\section{Exemplos}


\# Gerando uma amostra aleatória com

\# intercepto e inclinação aleatória em mu

\# e só intercepto em sigma

ransam $(\mathrm{N}=5, \mathrm{ni}=3, \mathrm{~b} 1=\mathrm{c}(2,-3), \mathrm{b} 2=\mathrm{c}(1,-3)$,

$\operatorname{sd} 10=1, \operatorname{sd} 11=0.9, \quad r h o 1=-0.5, \operatorname{sd} 20=0.9$,

param $=$ "WEI", p.cen $=0.30$ )

GHQ Gerador das combinações dos pontos de quadratura Gauss-Hermite

\section{Descrição}

Esta função permite obter todas as combinações de $k$ pontos de quadratura para solucionar uma integral $q$-dimensional.

\section{Uso}

GHQ (n.pontos, ndim, pruning = TRUE)

\section{Argumentos}

n.pontos número de pontos da quadratura.

ndim número de integrais ou dimensão da função a integrar.

pruning variável lógica, TRUE para eliminar combinações de pontos com pesos inferiores a $\theta$, FALSE para não realizar a eliminação.

\section{Detalhes}

Aquelas combinações de pontos com pesos inferiores ao valor $\theta$ são eliminadas porque essas combinações aportam poco a aproximação por meio da quadratura. Para mais detales vejase a seção 7.2 .

\section{Valores}

Obtém-se uma lista com três elementos, duas matrizes com as combinações de nós e pesos e um vetor com o produto dos pesos finais.

\section{Exemplos}

\# Sem eliminação

GHQ (n.pontos=2, ndim=3, pruning = FALSE)

\# Com eliminação

GHQ (n.pontos=2, ndim=3) 
summary função para obter a tabela de resumo

\section{Descrição}

Esta função permite obter uma tabela de resumo para objetos da classe weibullreg obtidos com a função wlmmML.

\section{Uso}

summary $(\bmod )$

\section{Argumentos}

mod modelo obtido com a função wlmmML.

\section{residual função para obter os resíduos}

\section{Descrição}

Função usada para obter os resíduos $r_{o}, r_{q}, r_{d}$ e $r_{M}$.

Uso

residual (mod)

\section{Argumentos}

mod modelo obtido com a função wlmmML.

\section{Valores}

Obtem-se uma lista com os seguintes elementos:

ro resíduo $r_{o}$.

rq resíduo $r_{q}$.

rM resíduo $r_{M}$.

rd resíduo $r_{d}$ 


\section{Descrição}

Função usada para predizer só os interceptos aleatórios no modelo multinível Weibull.

Uso

u.predict (mod)

\section{Argumentos}

mod modelo obtido com a função wlmmML.

\section{Valores}

Obtem-se uma matriz de duas colunas, a primeira para as predições dos interceptos aleatórios em $\mu$ e a segunda para as predições dos interceptos aleatórios em $\sigma$. 


\section{Capítulo 9}

\section{Conclusões}

Nesta tese foram apresentados os modelos multiníveis Weibull nos quais a parte sistemática dos dois parâmetros contém tanto efeitos fixos quanto interceptos aleatórios. Uma das novidades da proposta é a flexibilidade para a escolha da distribuição dos interceptos aleatórios. Pode-se escolher entre uma das seguintes quatro distribuições: normal, log-gama, logística e Cauchy. Uma outra extensão para os modelos multiníveis Weibull foi proposta na tese, que consistiu em incluir intercepto e inclinação aleatórias com distribuição normal bivariada nos dois parâmetros da distribuição. Um aspecto a destacar é a possíbilidade da inclusão de observações censuradas, o qual transforma estes modelos numa ferramenta útil para a análise de dados de sobrevivência. Adicionalmente, a definição do modelo permite a análise de dados em três parametrizações encontradas na literatura para a distribuição Weibull.

Para os modelos multiníveis Weibull foram propostos três tipos de resíduos: o resíduo ordinário padronizado, o resíduo componente do desvio e o resíduo quantil normal aleatorizado. Por meio de um estudo de simulação Monte Carlo concluiu-se que só os dois últimos resíduos têm distribuição empírica próxima da normal quando o modelo é corretamente especificado.

Uma característica diferenciada dos modelos propostos é que a estimação do vetor de parâmetros, composto pelos efeitos fixos e as componentes de variância, é realizada em forma conjunta pelo método de máxima verossimilhança com o uso da quadratura de Gauss Hermite para aproximar a função de verossimilhança. A quadratura de Gauss-Hermite foi considerada nas versões usual e adaptativa e numa nova proposta melhorada mais eficiente em termos de tempo computacional. Todos os procedimentos computacionais nos modelos propostos tais como estimação de parâmetros, predição dos efeitos aleatórios, obtenção dos resíduos e resumo de resultados foram desenvolvidos na linguagem $\mathrm{R}$ e organizados no pacote 'wlmm' para um possível uso por parte de pesquisadores interessados nos modelos ou nos procedimentos internos.

Para os modelos propostos sugere-se usar o teste da razão de verossimilhanças (RV) para decidir se é apropriado ou não a inclusão de interceptos aleatórios. Através de um 
estudo de simulação de Monte Carlo com os modelos multiníveis com interceptos aleatórios com distribuição normal, obteve-se que a distribuição empírica da estatística do teste RV é próxima de uma mistura 0,5:0,5 de distribuições $\chi_{0}^{2}$ e $\chi_{1}^{2}$.

Devido ao grande interesse encontrado na literatura em relação ao impacto de assumir incorretamente a distribuição dos efeitos aleatórios nos modelos lineares generalizados mistos, na tese foi desenvolvido um estudo de simulação na mesma direção para contribuir ao estudo desse problema. No estudo considerou-se o modelo multiníveis Weibull em que os interceptos aleatórios foram gerados com uma de seis distribuições, mas obtidas as estimativas dos parâmetros assumindo que a distribuição dos interceptos aleatórios é normal. Dos resultados foi encontrado impacto só nas estimativas das componentes de variância quando a verdadeira distribuição dos efeitos aleatórios foi assimétrica.

Como possíveis trabalhos futuros pode-se citar entre outros:

- Ampliar o estudo de simulação para estudar a distribuição empírica da estatística do teste RV nos modelos multiníveis com interceptos aleatórios não normais e nos modelos multiníveis com intercepto e inclinação aleatórias.

- Utilizar o teste do tipo escore unilateral para estudar a inclusão de interceptos aleatórios na modelagem nos parâmetros dos modelos multiníveis Weibull, assim como Savalli et al. (2006) e Russo et al. (2012) usaram o teste escore em modelos mistos lineares e não lineares, respectivamente.

- Estender o estudo da incorreta especificação da distribuição dos efeitos aleatórios usando o enfoque de Neuhaus et al. (2011), que consiste em gerar os efeitos aleatórios com uma distribuição fixa e obter as estimativas dos parâmetros do modelo assumindo várias distribuições para estes. 


\section{Apêndice A}

\section{Parametrizações da distribuição Weibull}

A distribuição Weibull proposta por Weibull (1939) é uma das distribuições com ampla aplicabilidade em estudos biomédicos e industriais. A popularidade de seu uso se deve ao fato de la apresentar uma grande variedade de formas e com taxa de falhas monótona (Colosimo e Giolo, 2006). Na literatura estatística é possível encontrar várias parametrizações da distribuição e neste apêndice apresentam-se as três versões usadas no presente trabalho. Na sequência são dadas expresões para a função de densidade, esperança, variância, função de distribuição acumulada e taxa de falha. As três parametrizações são denotadas respectivamente por $W E I, W E I 2$ e $W E I 3$. A primeira delas corresponde à parametrização usual da distribuição Weibull.

Parametrização $W E I$

$$
\begin{aligned}
f_{Y}(y \mid \mu, \sigma) & =\frac{\sigma y^{\sigma-1}}{\mu^{\sigma}} \exp \left[-\left(\frac{y}{\mu}\right)^{\sigma}\right], \quad \mu>0, \quad \sigma>0 \\
E(Y) & =\mu \Gamma\left(\frac{1}{\sigma}+1\right) \\
\operatorname{Var}(Y) & =\mu^{2}\left[\Gamma\left(\frac{2}{\sigma}+1\right)-\Gamma^{2}\left(\frac{1}{\sigma}+1\right)\right] \\
F(y) & =1-\exp \left[-\left(\frac{y}{\mu}\right)^{\sigma}\right] \\
\text { e } h(y) & =\frac{\sigma y^{\sigma-1}}{\mu^{\sigma}}
\end{aligned}
$$


Parametrização WEI2

$$
\begin{aligned}
f_{Y}(y \mid \mu, \sigma) & =\sigma \mu y^{\sigma-1} \exp \left(-\mu y^{\sigma}\right), \quad \mu>0, \quad \sigma>0, \\
E(Y) & =\mu^{-1 / \sigma} \Gamma\left(\frac{1}{\sigma}+1\right), \\
\operatorname{Var}(Y) & =\mu^{-2 / \sigma}\left[\Gamma\left(\frac{2}{\sigma}+1\right)-\Gamma^{2}\left(\frac{1}{\sigma}+1\right)\right], \\
F(y) & =1-\exp \left(-\mu y^{\sigma}\right), \\
\text { e } \quad h(y) & =\sigma \mu y^{\sigma-1} .
\end{aligned}
$$

Parametrização WEI3

$$
\begin{aligned}
f_{Y}(y \mid \mu, \sigma) & =\frac{\sigma}{\kappa}\left(\frac{y}{\kappa}\right)^{\sigma-1} \exp \left[-\left(\frac{y}{\kappa}\right)^{\sigma}\right], \mu>0, \quad \sigma>0, \\
E(Y) & =\mu, \\
\operatorname{Var}(Y) & =\mu^{2}\left[\Gamma\left(\frac{2}{\sigma}+1\right) \Gamma^{-2}\left(\frac{1}{\sigma}+1\right)-1\right], \\
F(y) & =1-\exp \left[-\left(\frac{y}{\kappa}\right)^{\sigma}\right], \\
\text { e } h(y) & =\frac{\sigma}{\kappa}\left(\frac{y}{\kappa}\right)^{\sigma-1},
\end{aligned}
$$

$\operatorname{com} \kappa=\mu / \Gamma(1 / \sigma+1)$.

Nas Figuras A.1 a A.3 apresentam-se os gráficos das funções de densidade, sobrevivência e taxa de falhas nas três versões para diferentes valores dos parâmetros $\mu$ e $\sigma$. Das figuras observa-se que a distribuição pode tomar formas diferentes e que a taxa de falhas $h(y)$ é sempre crescente, decrescente ou constante.
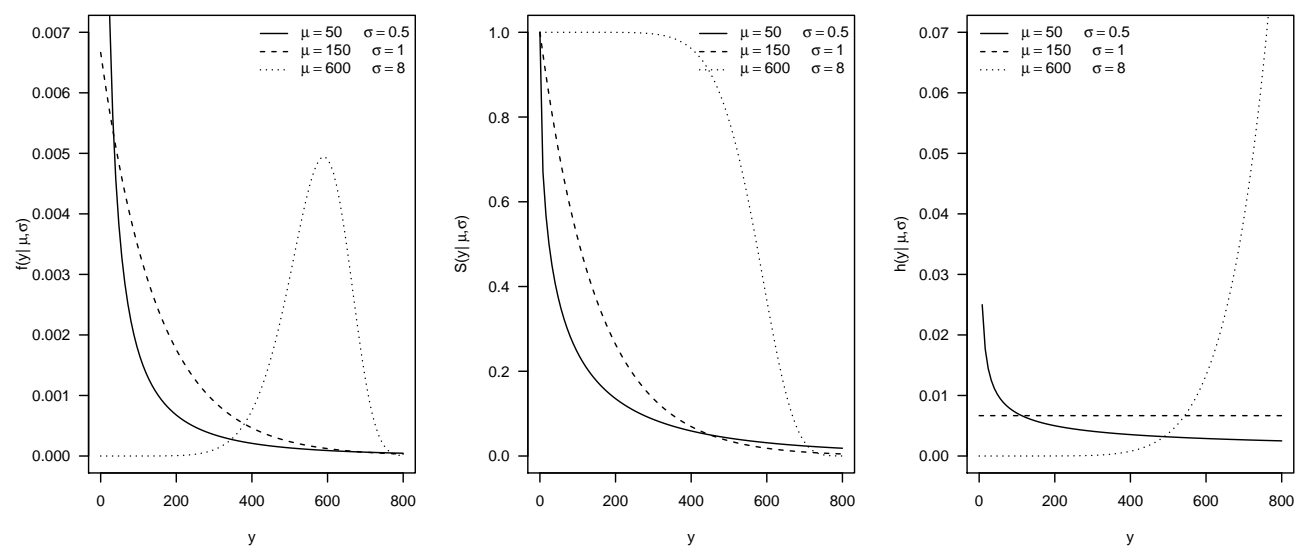

Figura A.1: Funções de densidade, sobrevivência e risco da parameterização $W E I$ para diferentes valores de $\mu$ e $\sigma$. 

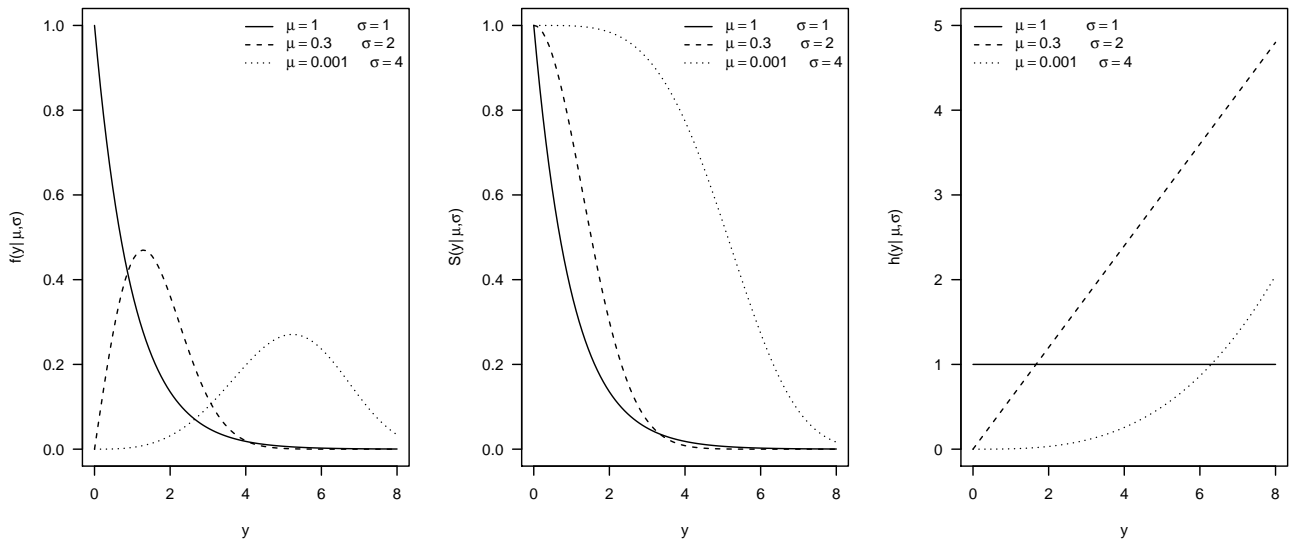

Figura A.2: Funçôes de densidade, sobrevivência e risco da parameterização WEI2 para diferentes valores de $\mu$ e $\sigma$.
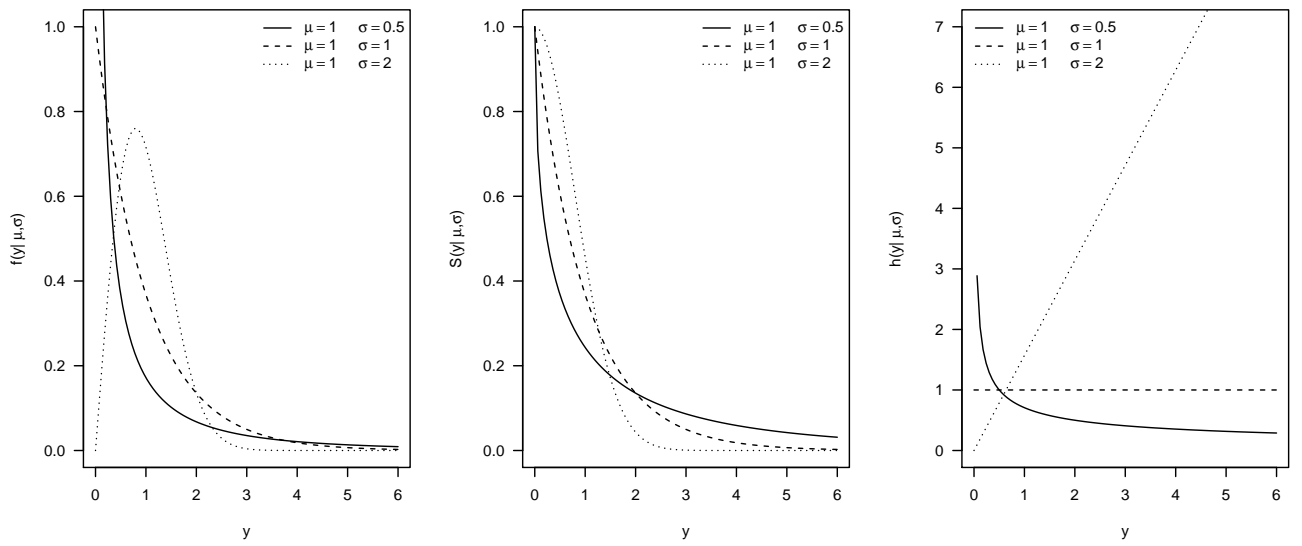

Figura A.3: Funçôes de densidade, sobrevivência e risco da parameterização $W E I 3$ para diferentes valores de $\mu$ e $\sigma$. 
APÊNDICE A 


\section{Apêndice B}

\section{Bases de dados}

Tabela B.1: Base de dados simulada multiniveis com dois niveis, resposta Weibull e efeitos aleatórios normais.

\begin{tabular}{ccccc|ccccc}
\hline Obs. & $\mathrm{y}$ & $x_{1}$ & $x_{2}$ & Grupo & Obs. & $\mathrm{y}$ & $x_{1}$ & $x_{2}$ & Grupo \\
\hline 1 & 0,63 & 2,20 & $-1,28$ & 1 & 26 & 1,24 & 1,90 & $-1,07$ & 6 \\
2 & 0,34 & 1,70 & $-0,69$ & 1 & 27 & 0,13 & 1,74 & $-1,08$ & 6 \\
3 & 0,00 & 1,57 & $-1,27$ & 1 & 28 & 0,76 & 1,74 & $-1,30$ & 6 \\
4 & 0,09 & 2,01 & $-0,82$ & 1 & 29 & 0,05 & 1,53 & $-0,97$ & 6 \\
5 & 0,00 & 1,86 & $-0,90$ & 1 & 30 & 1,54 & 2,30 & $-1,17$ & 6 \\
6 & 1,52 & 2,38 & $-0,46$ & 2 & 31 & 0,50 & 2,02 & $-1,05$ & 7 \\
7 & 1,48 & 1,91 & $-1,07$ & 2 & 32 & 1,57 & 1,98 & $-0,89$ & 7 \\
8 & 1,34 & 1,94 & $-0,85$ & 2 & 33 & 1,32 & 2,35 & $-0,85$ & 7 \\
9 & 0,85 & 2,06 & $-0,71$ & 2 & 34 & 1,08 & 1,58 & $-0,87$ & 7 \\
10 & 1,44 & 2,29 & $-1,06$ & 2 & 35 & 0,85 & 2,33 & $-1,54$ & 7 \\
11 & 0,51 & 1,94 & $-0,74$ & 3 & 36 & 0,20 & 2,12 & $-1,43$ & 8 \\
12 & 0,77 & 2,11 & $-1,02$ & 3 & 37 & 0,59 & 1,98 & $-1,14$ & 8 \\
13 & 0,78 & 1,89 & $-1,03$ & 3 & 38 & 0,93 & 1,82 & $-0,96$ & 8 \\
14 & 0,82 & 2,31 & $-0,91$ & 3 & 39 & 1,34 & 2,34 & $-1,00$ & 8 \\
15 & 1,00 & 2,27 & $-0,80$ & 3 & 40 & 1,05 & 2,04 & $-1,02$ & 8 \\
16 & 0,45 & 1,92 & $-0,89$ & 4 & 41 & 0,33 & 1,89 & $-0,81$ & 9 \\
17 & 0,16 & 1,64 & $-1,28$ & 4 & 42 & 0,27 & 2,43 & $-1,04$ & 9 \\
18 & 0,67 & 1,48 & $-1,43$ & 4 & 43 & 0,14 & 2,17 & $-0,36$ & 9 \\
19 & 0,41 & 1,93 & $-0,06$ & 4 & 44 & 0,11 & 1,46 & $-0,81$ & 9 \\
20 & 1,00 & 1,78 & $-1,06$ & 4 & 45 & 0,11 & 2,03 & $-0,99$ & 9 \\
21 & 0,32 & 2,30 & $-1,33$ & 5 & 46 & 0,18 & 1,63 & $-0,81$ & 10 \\
22 & 0,35 & 1,69 & $-0,58$ & 5 & 47 & 0,66 & 2,16 & $-0,85$ & 10 \\
23 & 0,21 & 1,68 & $-1,15$ & 5 & 48 & 0,93 & 2,47 & $-0,75$ & 10 \\
24 & 0,32 & 2,04 & $-0,77$ & 5 & 49 & 0,83 & 2,44 & $-0,99$ & 10 \\
25 & 0,28 & 1,91 & $-0,33$ & 5 & 50 & 1,14 & 2,04 & $-0,95$ & 10 \\
\hline
\end{tabular}


Tabela B.2: Base de dados simulada multinível com dois níveis, resposta Weibull e efeitos aleatórios log-gama.

\begin{tabular}{|c|c|c|c|c|c|c|c|c|c|}
\hline Obs. & Grupo & $\mathrm{y}$ & $x_{1}$ & $x_{2}$ & Obs. & Grupo & $\mathrm{y}$ & $x_{1}$ & $x_{2}$ \\
\hline 1 & 1 & 257,20 & 2,10 & $-0,87$ & 51 & 6 & 134,92 & 1,81 & $-1,12$ \\
\hline 2 & 1 & 184,93 & 1,92 & $-1,08$ & 52 & 6 & 180,53 & 1,95 & $-1,18$ \\
\hline 3 & 1 & 244,03 & 2,04 & $-1,19$ & 53 & 6 & 301,60 & 2,20 & $-0,96$ \\
\hline 4 & 1 & 309,08 & 2,19 & $-1,44$ & 54 & 6 & 156,07 & 1,88 & $-1,01$ \\
\hline 5 & 1 & 242,97 & 2,05 & $-1,16$ & 55 & 6 & 116,96 & 1,76 & $-1,14$ \\
\hline 6 & 1 & 413,59 & 2,37 & $-0,89$ & 56 & 6 & 133,34 & 1,80 & $-1,23$ \\
\hline 7 & 1 & 193,67 & 1,92 & $-1,14$ & 57 & 6 & 143,03 & 1,80 & $-0,74$ \\
\hline 8 & 1 & 245,79 & 2,03 & $-1,10$ & 58 & 6 & 121,25 & 1,83 & $-1,12$ \\
\hline 9 & 1 & 280,60 & 2,11 & $-0,67$ & 59 & 6 & 199,43 & 2,00 & $-0,85$ \\
\hline 10 & 1 & 148,56 & 1,86 & $-1,02$ & 60 & 6 & 161,37 & 1,93 & $-0,73$ \\
\hline 11 & 2 & 124,22 & 1,72 & $-1,25$ & 61 & 7 & 210,19 & 2,15 & $-1,25$ \\
\hline 12 & 2 & 227,95 & 2,01 & $-1,24$ & 62 & 7 & 168,64 & 2,05 & $-1,03$ \\
\hline 13 & 2 & 176,08 & 1,90 & $-1,17$ & 63 & 7 & 115,08 & 1,88 & $-1,14$ \\
\hline 14 & 2 & 293,22 & 2,10 & $-0,84$ & 64 & 7 & 273,27 & 2,27 & $-0,75$ \\
\hline 15 & 2 & 291,15 & 2,14 & $-1,27$ & 65 & 7 & 186,60 & 2,14 & $-1,24$ \\
\hline 16 & 2 & 189,41 & 1,89 & $-0,90$ & 66 & 7 & 62,95 & 1,60 & $-0,96$ \\
\hline 17 & 2 & 183,29 & 1,90 & $-0,87$ & 67 & 7 & 258,52 & 2,23 & $-0,87$ \\
\hline 18 & 2 & 210,18 & 1,93 & $-0,75$ & 68 & 7 & 375,76 & 2,45 & $-1,08$ \\
\hline 19 & 2 & 512,30 & 2,44 & $-1,14$ & 69 & 7 & 321,76 & 2,39 & $-1,00$ \\
\hline 20 & 2 & 230,14 & 2,00 & $-1,02$ & 70 & 7 & 219,27 & 2,21 & $-0,89$ \\
\hline 21 & 3 & 155,75 & 1,76 & $-1,13$ & 71 & 8 & 154,61 & 1,99 & $-0,76$ \\
\hline 22 & 3 & 152,99 & 1,74 & $-1,31$ & 72 & 8 & 94,08 & 2,08 & $-0,68$ \\
\hline 23 & 3 & 180,48 & 1,81 & $-1,22$ & 73 & 8 & 62,09 & 1,99 & $-0,89$ \\
\hline 24 & 3 & 284,29 & 2,12 & $-1,23$ & 74 & 8 & 114,60 & 2,11 & $-1,25$ \\
\hline 25 & 3 & 318,11 & 2,09 & $-1,11$ & 75 & 8 & 100,71 & 1,87 & $-0,83$ \\
\hline 26 & 3 & 226,63 & 2,00 & $-0,77$ & 76 & 8 & 75,96 & 2,02 & $-1,39$ \\
\hline 27 & 3 & 149,82 & 1,73 & $-1,16$ & 77 & 8 & 118,00 & 1,98 & $-0,87$ \\
\hline 28 & 3 & 389,97 & 2,19 & $-1,12$ & 78 & 8 & 115,48 & 2,08 & $-0,79$ \\
\hline 29 & 3 & 188,69 & 1,82 & $-1,14$ & 79 & 8 & 200,83 & 2,38 & $-1,07$ \\
\hline 30 & 3 & 162,55 & 1,85 & $-1,21$ & 80 & 8 & 186,92 & 2,18 & $-0,78$ \\
\hline 31 & 4 & 96,02 & 2,15 & $-1,04$ & 81 & 9 & 10,10 & 1,81 & $-0,95$ \\
\hline 32 & 4 & 116,01 & 2,31 & $-0,71$ & 82 & 9 & 20,68 & 2,07 & $-1,09$ \\
\hline 33 & 4 & 107,10 & 2,22 & $-1,29$ & 83 & 9 & 15,38 & 1,92 & $-0,96$ \\
\hline 34 & 4 & 64,72 & 2,00 & $-1,13$ & 84 & 9 & 7,01 & 1,59 & $-0,98$ \\
\hline 35 & 4 & 27,55 & 1,56 & $-1,39$ & 85 & 9 & 19,39 & 2,07 & $-0,80$ \\
\hline 36 & 4 & 99,50 & 2,21 & $-1,08$ & 86 & 9 & 16,65 & 1,93 & $-1,04$ \\
\hline 37 & 4 & 112,33 & 2,21 & $-0,96$ & 87 & 9 & 11,49 & 1,85 & $-1,00$ \\
\hline 38 & 4 & 34,51 & 1,64 & $-0,73$ & 88 & 9 & 13,82 & 1,90 & $-1,12$ \\
\hline 39 & 4 & 59,55 & 1,94 & $-0,82$ & 89 & 9 & 16,36 & 2,01 & $-1,08$ \\
\hline 40 & 4 & 60,32 & 1,93 & $-1,01$ & 90 & 9 & 18,92 & 2,05 & $-1,03$ \\
\hline 41 & 5 & 44,76 & 1,85 & $-1,10$ & 91 & 10 & 123,63 & 2,09 & $-1,26$ \\
\hline 42 & 5 & 50,93 & 2,00 & $-1,03$ & 92 & 10 & 44,77 & 1,74 & $-0,76$ \\
\hline 43 & 5 & 73,47 & 2,09 & $-0,99$ & 93 & 10 & 62,69 & 1,78 & $-1,07$ \\
\hline 44 & 5 & 52,56 & 1,94 & $-1,13$ & 94 & 10 & 62,52 & 1,75 & $-0,91$ \\
\hline 45 & 5 & 106,87 & 2,28 & $-0,98$ & 95 & 10 & 98,57 & 1,98 & $-0,86$ \\
\hline 46 & 5 & 71,72 & 2,09 & $-1,14$ & 96 & 10 & 164,47 & 2,23 & $-1,22$ \\
\hline 47 & 5 & 58,04 & 1,91 & $-0,69$ & 97 & 10 & 42,98 & 1,58 & $-1,43$ \\
\hline 48 & 5 & 74,55 & 2,11 & $-1,34$ & 98 & 10 & 61,13 & 1,72 & $-1,14$ \\
\hline 49 & 5 & 31,13 & 1,65 & $-1,02$ & 99 & 10 & 96,73 & 1,95 & $-0,84$ \\
\hline 50 & 5 & 97,21 & 2,19 & $-1,28$ & 100 & 10 & 83,97 & 1,91 & $-0,89$ \\
\hline
\end{tabular}




\section{Apêndice C}

\section{Função escore e matriz hessiana para o modelo Weibull com interceptos aleatórios normais}

A função densidade marginal aproximada para $\boldsymbol{y}_{i}$ no modelo multinível Weibull com efeitos aleatórios normais usando a aproximação QGH é dada por

$$
P\left(\boldsymbol{y}_{i}\right) \approx \sum_{l=1}^{N_{Q G H}} \sum_{m=1}^{N_{Q G H}} \exp \left[g\left(\boldsymbol{y}_{i}, \boldsymbol{\delta}_{i}, \sqrt{2} \boldsymbol{p}_{l, m}^{\top} \boldsymbol{T}\right)\right] \frac{w_{l} w_{m}}{\pi}
$$

em que $\boldsymbol{p}_{l, m}=\left(p_{l}, p_{m}\right)^{\top}$ e o logaritmo da função de verossimilhança fica expresso como

$$
l(\boldsymbol{\theta})=\sum_{i=1}^{N} \log P\left(\boldsymbol{y}_{i}\right)
$$

A função escore e a matriz hessiana são obtidas derivando $l(\boldsymbol{\theta})$ em relação às componentes de $\boldsymbol{\theta}$ como apresentado na sequência.

\section{C.1 Função escore}

Derivando $l(\boldsymbol{\theta})$ da expressão (3.14) com relação à $r$-ésima componente de $\boldsymbol{\beta}_{1}$ temos que

$$
\begin{aligned}
\frac{\partial l(\boldsymbol{\theta})}{\partial \boldsymbol{\beta}_{1 r}} & =\sum_{i=1}^{N} \frac{1}{P_{i}}\left[\sum_{l, m}^{N_{Q G H}} e^{g(\cdot)}\left[\boldsymbol{X}_{1 i, r}^{\top} \operatorname{diag}\left(\boldsymbol{A}_{i}\right) \boldsymbol{\delta}_{i}-\boldsymbol{X}_{1 i, r}^{\top} \operatorname{diag}\left(\boldsymbol{B}_{i}\right) \boldsymbol{y}_{i}^{\star}\right] \frac{w_{l} w_{m}}{\pi}\right] \\
& =\sum_{i=1}^{N} \frac{1}{P_{i}}\left[\sum_{l, m}^{N_{Q G H}} e^{g(\cdot)} \boldsymbol{X}_{1 i, r}^{\top}\left[\operatorname{diag}\left(\boldsymbol{A}_{i}\right) \boldsymbol{\delta}_{i}-\operatorname{diag}\left(\boldsymbol{B}_{i}\right) \boldsymbol{y}_{i}^{\star}\right] \frac{w_{l} w_{m}}{\pi}\right] \\
& =\sum_{i=1}^{N} \frac{\boldsymbol{X}_{1 i, r}^{\top} \boldsymbol{C}_{1 i}}{P_{i}}
\end{aligned}
$$


em que

$$
\begin{aligned}
\boldsymbol{A}_{i} & =\left(\frac{\partial \log \alpha_{i 1}}{\partial \eta_{1 i 1}}, \ldots, \frac{\partial \log \alpha_{i n_{i}}}{\partial \eta_{1 i n_{i}}}\right)^{\top}, \\
\boldsymbol{B}_{i} & =\left(\frac{\partial \alpha_{i 1}}{\partial \eta_{1 i 1}}, \ldots, \frac{\partial \alpha_{i n_{i}}}{\partial \eta_{1 i n_{i}}}\right)^{\top}, \\
\boldsymbol{C}_{1 i} & =\sum_{l=1}^{N_{Q G H}} \sum_{m=1}^{N_{Q G H}} e^{g(\cdot)} \boldsymbol{W}_{1} \frac{w_{l} w_{m}}{\pi}, \\
\boldsymbol{W}_{1} & =\operatorname{diag}\left(\boldsymbol{A}_{i}\right) \boldsymbol{\delta}_{i}-\operatorname{diag}\left(\boldsymbol{B}_{i}\right) \boldsymbol{y}_{i}^{\star}, \\
\boldsymbol{y}_{i}^{\star} & =\left(y_{i 1}^{\sigma_{i 1}}, y_{i 2}^{\sigma_{i 2}}, \ldots, y_{i n_{i}}^{\sigma_{i n_{i}}}\right)^{\top},
\end{aligned}
$$

com $g(\cdot)=g\left(\boldsymbol{y}_{i}, \boldsymbol{\delta}_{i}, \sqrt{2} \boldsymbol{p}_{l, m}^{\top} \boldsymbol{T}\right)$ dada pela expressão (3.9) e diag $(\cdot)$ uma matriz diagonal. A derivada para $l(\boldsymbol{\theta})$ com relação ao vetor $\boldsymbol{\beta}_{1}$ é dada por

$$
\frac{\partial l(\boldsymbol{\theta})}{\partial \boldsymbol{\beta}_{1}}=\sum_{i=1}^{N} \frac{\boldsymbol{X}_{1 i}^{\top} \boldsymbol{C}_{1 i}}{P_{i}}
$$

Derivando $l(\boldsymbol{\theta})$ em relação à $s$-ésima componente de $\boldsymbol{\beta}_{2}$ temos que:

$$
\begin{aligned}
\frac{\partial l(\boldsymbol{\theta})}{\partial \boldsymbol{\beta}_{2 s}} & =\sum_{i=1}^{N} \frac{1}{P_{i}}\left[\sum_{l, m}^{N_{Q G H}} e^{g(\cdot)} \boldsymbol{X}_{2 i, s}^{\top}\left[\boldsymbol{\delta}_{i}+\operatorname{diag}\left(\boldsymbol{D}_{i}\right) \boldsymbol{\delta}_{i}+\operatorname{diag}\left(\boldsymbol{\sigma}_{i}^{\star}\right) \log \left(\boldsymbol{y}_{i}\right)-\boldsymbol{E}_{i}\right] \frac{w_{l} w_{m}}{\pi}\right] \\
& =\sum_{i=1}^{N} \frac{\boldsymbol{X}_{2 i, s}^{\top} \boldsymbol{C}_{2 i}}{P_{i}}
\end{aligned}
$$

em que

$$
\begin{aligned}
\boldsymbol{D}_{i} & =\left(\frac{\partial \log \alpha_{i 1}}{\partial \eta_{2 i 1}}, \ldots, \frac{\partial \log \alpha_{i n_{i}}}{\partial \eta_{2 i n_{i}}}\right)^{\top}, \\
\boldsymbol{E}_{i} & =\left(\frac{\partial \alpha_{i 1} y_{i 1}^{\sigma_{i 1}}}{\partial \eta_{2 i 1}}, \ldots, \frac{\partial \alpha_{i n_{i}} y_{i n_{i}}}{\partial \eta_{2 i n_{i}}}\right)^{\top}, \\
\boldsymbol{C}_{2 i} & =\sum_{l=1}^{N_{Q G H}} \sum_{m=1}^{N_{Q G H}} e^{g(\cdot)} \boldsymbol{W}_{2} \frac{w_{l} w_{m}}{\pi}, \\
\boldsymbol{W}_{2} & =\boldsymbol{\delta}_{i}+\operatorname{diag}\left(\boldsymbol{D}_{i}\right) \boldsymbol{\delta}_{i}+\operatorname{diag}\left(\boldsymbol{\sigma}_{i}^{\star}\right) \log \left(\boldsymbol{y}_{i}\right)-\boldsymbol{E}_{i}, \\
\boldsymbol{\sigma}_{i}^{\star} & =\left(\delta_{i 1} \sigma_{i 1}, \ldots, \delta_{i n_{i}} \sigma_{i n_{i}}\right)^{\top} .
\end{aligned}
$$

A derivada para $l(\boldsymbol{\theta})$ em relação ao vetor $\boldsymbol{\beta}_{2}$ é dada por

$$
\frac{\partial l(\boldsymbol{\theta})}{\partial \boldsymbol{\beta}_{2}}=\sum_{i=1}^{N} \frac{\boldsymbol{X}_{2 i}^{\top} \boldsymbol{C}_{2 i}}{P_{i}}
$$


Derivando $l(\boldsymbol{\theta})$ em relação $\tau_{1}$ temos que

$$
\begin{aligned}
\frac{\partial l(\boldsymbol{\theta})}{\partial \tau_{1}} & =\sum_{i=1}^{N} \frac{1}{P_{i}}\left[\sum_{l, m}^{N_{Q G H}} e^{g(\cdot)} \sqrt{2} p_{l}\left[\boldsymbol{\delta}_{i}^{\top} \boldsymbol{A}_{i}-\boldsymbol{B}_{i}^{\top} \boldsymbol{y}_{i}^{\star}\right] \frac{w_{l} w_{m}}{\pi}\right] \\
& =\sum_{i=1}^{N} \frac{C_{3 i}}{P_{i}}
\end{aligned}
$$

em que

$$
\begin{aligned}
C_{3 i} & =\sum_{l=1}^{N_{Q G H}} \sum_{m=1}^{N_{Q G H}} e^{g(\cdot)} W_{3} \frac{w_{l} w_{m}}{\pi} \sqrt{2} p_{l}, \\
W_{3} & =\boldsymbol{\delta}_{i}^{\top} \boldsymbol{A}_{i}-\boldsymbol{B}_{i}^{\top} \boldsymbol{y}_{i}^{\star} .
\end{aligned}
$$

Derivando $l(\boldsymbol{\theta})$ em relação $\tau_{2}$ temos que

$$
\begin{aligned}
\frac{\partial l(\boldsymbol{\theta})}{\partial \tau_{2}} & =\sum_{i=1}^{N} \frac{1}{P_{i}}\left[\sum_{l, m}^{N_{Q G H}} e^{g(\cdot)} \sqrt{2} p_{m}\left[\boldsymbol{\delta}_{i}^{\top} \mathbf{1}_{n i}+\boldsymbol{\delta}_{i}^{\top} \boldsymbol{D}_{i}+\boldsymbol{\sigma}_{i}^{\star T} \log \left(\boldsymbol{y}_{i}\right)-\boldsymbol{E}_{i}^{\top} \mathbf{1}_{n_{i}}\right] \frac{w_{l} w_{m}}{\pi}\right] \\
& =\sum_{i=1}^{N} \frac{C_{4 i}}{P_{i}}
\end{aligned}
$$

em que

$$
\begin{aligned}
C_{4 i} & =\sum_{l=1}^{N_{Q G H}} \sum_{m=1}^{N_{Q G H}} e^{g(\cdot)} W_{4} \frac{w_{l} w_{m}}{\pi} \sqrt{2} p_{m}, \\
W_{4} & =\boldsymbol{\delta}_{i}^{\top} \mathbf{1}_{n i}+\boldsymbol{\delta}_{i}^{\top} \boldsymbol{D}_{i}+\boldsymbol{\sigma}_{i}^{\star T} \log \left(\boldsymbol{y}_{i}\right)-\boldsymbol{E}_{i}^{\top} \mathbf{1}_{n_{i}} .
\end{aligned}
$$

\section{C.2 Matriz hessiana}

Derivando $l(\boldsymbol{\theta})$ da expressão (3.14) duas vezes, primeiro com relação à $r$-ésima componente de $\boldsymbol{\beta}_{1}$ e em seguida com relação à s-ésima componente de $\boldsymbol{\beta}_{2}$ temos que

$$
\frac{\partial^{2} l(\boldsymbol{\theta})}{\partial \boldsymbol{\beta}_{1 s} \partial \boldsymbol{\beta}_{1 r}}=\sum_{i=1}^{N}\left[\frac{-\left(\boldsymbol{X}_{1 i, r}^{\top} \boldsymbol{C}_{1 i}\right)\left(\boldsymbol{X}_{1 i, \cdot s}^{\top} \boldsymbol{C}_{1 i}\right)}{P_{i}^{2}}+\frac{\boldsymbol{X}_{1 i, r}^{\top} \boldsymbol{H}_{1 i} \boldsymbol{X}_{1 i, s}}{P_{i}}\right],
$$


em que

$$
\begin{aligned}
\boldsymbol{H}_{1 i} & =\sum_{l=1}^{N_{Q G H}} \sum_{m=1}^{N_{Q G H}} e^{g(\cdot)}\left[\boldsymbol{W}_{1} \boldsymbol{W}_{1}^{\top}+\operatorname{diag}\left(\boldsymbol{F}_{i}-\boldsymbol{G}_{i}\right)\right] \frac{w_{l} w_{m}}{\pi}, \\
\boldsymbol{F}_{i} & =\left(\delta_{i 1} \frac{\partial A_{i 1}}{\partial \eta_{1 i 1}}+\ldots+\delta_{i n_{i}} \frac{\partial A_{i n_{i}}}{\partial \eta_{1 i n_{i}}}\right)^{\top}, \\
\boldsymbol{G}_{i} & =\left(y_{i 1}^{\star} \frac{\partial B_{i 1}}{\partial \eta_{1 i 1}}+\ldots+y_{i n_{i}}^{\star} \frac{\partial B_{i n_{i}}}{\partial \eta_{1 i n_{i}}}\right)^{\top} .
\end{aligned}
$$

Assim, a matriz obtida ao derivar $l(\boldsymbol{\theta})$ duas vezes em relação ao vetor $\boldsymbol{\beta}_{1}$ é a seguinte:

$$
\frac{\partial^{2} l(\boldsymbol{\theta})}{\partial \boldsymbol{\beta}_{1}^{2}}=\sum_{i=1}^{N} \boldsymbol{X}_{1 i}^{\top}\left[\frac{-\boldsymbol{C}_{1 i} \boldsymbol{C}_{1 i}^{\top}}{P_{i}^{2}}+\frac{\boldsymbol{H}_{1 i}}{P_{i}}\right] \boldsymbol{X}_{1 i}
$$

Derivando $l(\boldsymbol{\theta})$ duas vezes, primeiro em relação à $r$-ésima componente de $\boldsymbol{\beta}_{1}$ e depois em relação à $s$-ésima componente de $\boldsymbol{\beta}_{2}$, temos que

$$
\frac{\partial^{2} l(\boldsymbol{\theta})}{\partial \boldsymbol{\beta}_{2 s} \partial \boldsymbol{\beta}_{1 r}}=\sum_{i=1}^{N}\left[\frac{-\left(\boldsymbol{X}_{1 i, r}^{\top} \boldsymbol{C}_{1 i}\right)\left(\boldsymbol{X}_{2 i, s}^{\top} \boldsymbol{C}_{2 i}\right)}{P_{i}^{2}}+\frac{\boldsymbol{X}_{1 i, r}^{\top} \boldsymbol{H}_{2 i} \boldsymbol{X}_{2 i, s}}{P_{i}}\right],
$$

em que

$$
\begin{aligned}
\boldsymbol{H}_{2 i} & =\sum_{l=1}^{N_{Q G H}} \sum_{m=1}^{N_{Q G H}} e^{g(\cdot)}\left[\boldsymbol{W}_{1} \boldsymbol{W}_{2}^{\top}+\operatorname{diag}\left(\boldsymbol{J}_{i}-\boldsymbol{I}_{i}\right)\right] \frac{w_{l} w_{m}}{\pi}, \\
\boldsymbol{J}_{i} & =\left(\delta_{i 1} \frac{\partial A_{i 1}}{\partial \eta_{2 i 1}}, \ldots, \delta_{i n_{i}} \frac{\partial A_{i n_{i}}}{\partial \eta_{2 i n_{i}}}\right)^{\top}, \\
\boldsymbol{I}_{i} & =\left(\frac{\partial\left(y_{i 1}^{\star} B_{i 1}\right)}{\partial \eta_{2 i 1}}, \ldots, \frac{\partial\left(y_{i n_{i}}^{\star} B_{i n_{i}}\right)}{\partial \eta_{2 i n_{i}}}\right)^{\top} .
\end{aligned}
$$

Assim, a matriz obtida ao derivar $l(\boldsymbol{\theta})$ duas vezes, primeiro em relação a $\boldsymbol{\beta}_{2}$ e depois em relação a $\boldsymbol{\beta}_{1}$, é a seguinte:

$$
\frac{\partial^{2} l(\boldsymbol{\theta})}{\partial \boldsymbol{\beta}_{2} \partial \boldsymbol{\beta}_{1}}=\sum_{i=1}^{N} \boldsymbol{X}_{1 i}^{\top}\left[\frac{-\boldsymbol{C}_{1 i} \boldsymbol{C}_{2 i}^{\top}}{P_{i}^{2}}+\frac{\boldsymbol{H}_{2 i}}{P_{i}}\right] \boldsymbol{X}_{2 i}
$$

Derivando $l(\boldsymbol{\theta})$ duas vezes, primeiro em relação à $r$-ésima componente de $\boldsymbol{\beta}_{1}$ e depois em relação a $\tau_{1}$, temos que

$$
\frac{\partial^{2} l(\boldsymbol{\theta})}{\partial \tau_{1} \partial \boldsymbol{\beta}_{1 r}}=\sum_{i=1}^{N}\left[\frac{-\boldsymbol{X}_{1 i, r}^{\top} \boldsymbol{C}_{1 i} C_{3 i}}{P_{i}^{2}}+\frac{\boldsymbol{X}_{1 i, r}^{\top} \boldsymbol{H}_{3 i}}{P_{i}}\right]
$$


em que

$$
\boldsymbol{H}_{3 i}=\sum_{l=1}^{N_{Q G H}} \sum_{m=1}^{N_{Q G H}} e^{g(\cdot)}\left[W_{3} \boldsymbol{W}_{1}+\boldsymbol{F}_{i}-\boldsymbol{G}_{i}\right] \frac{w_{l} w_{m} \sqrt{2} p_{l}}{\pi} .
$$

Assim, a matriz obtida ao derivar $l(\boldsymbol{\theta})$ duas vezes, primeiro em relação a $\boldsymbol{\beta}_{1}$ e depois em relação a $\tau_{1}$, é a seguinte:

$$
\frac{\partial^{2} l(\boldsymbol{\theta})}{\partial \tau_{1} \partial \boldsymbol{\beta}_{1}}=\sum_{i=1}^{N} \boldsymbol{X}_{1 i}^{\top}\left[\frac{-\boldsymbol{C}_{1 i} C_{3 i}}{P_{i}^{2}}+\frac{\boldsymbol{H}_{3 i}}{P_{i}}\right]
$$

Derivando $l(\boldsymbol{\theta})$ duas vezes, primeiro em relação à $r$-ésima componente de $\boldsymbol{\beta}_{1}$ e depois em relação a $\tau_{2}$, temos que

$$
\frac{\partial^{2} l(\boldsymbol{\theta})}{\partial \tau_{2} \partial \boldsymbol{\beta}_{1 r}}=\sum_{i=1}^{N}\left[\frac{-\boldsymbol{X}_{1 i, r}^{\top} \boldsymbol{C}_{1 i} C_{4 i}}{P_{i}^{2}}+\frac{\boldsymbol{X}_{1 i, \cdot r}^{\top} \boldsymbol{H}_{4 i}}{P_{i}}\right]
$$

em que

$$
\boldsymbol{H}_{4 i}=\sum_{l=1}^{N_{Q G H}} \sum_{m=1}^{N_{Q G H}} e^{g(\cdot)}\left[W_{4} \boldsymbol{W}_{1}+\boldsymbol{J}_{i}-\boldsymbol{I}_{i}\right] \frac{w_{l} w_{m} \sqrt{2} p_{m}}{\pi} .
$$

Assim, a matriz obtida ao derivar $l(\boldsymbol{\theta})$ duas vezes, primeiro em relação a $\boldsymbol{\beta}_{1}$ e depois em relação a $\tau_{2}$, é a seguinte:

$$
\frac{\partial^{2} l(\boldsymbol{\theta})}{\partial \tau_{2} \partial \boldsymbol{\beta}_{1}}=\sum_{i=1}^{N} \boldsymbol{X}_{1 i}^{\top}\left[\frac{-\boldsymbol{C}_{1 i} C_{4 i}}{P_{i}^{2}}+\frac{\boldsymbol{H}_{4 i}}{P_{i}}\right]
$$

Derivando $l(\boldsymbol{\theta})$ duas vezes, primeiro em relação à $r$-ésima componente de $\boldsymbol{\beta}_{2}$ e depois em relação à $s$-ésima componente de $\boldsymbol{\beta}_{2}$, temos que

$$
\frac{\partial^{2} l(\boldsymbol{\theta})}{\partial \boldsymbol{\beta}_{2 s} \partial \boldsymbol{\beta}_{2 r}}=\sum_{i=1}^{N}\left[\frac{-\left(\boldsymbol{X}_{2 i, r}^{\top} \boldsymbol{C}_{2 i}\right)\left(\boldsymbol{X}_{2 i, \cdot s}^{\top} \boldsymbol{C}_{2 i}\right)}{P_{i}^{2}}+\frac{\boldsymbol{X}_{2 i, r}^{\top} \boldsymbol{H}_{5 i} \boldsymbol{X}_{2 i, \cdot s}}{P_{i}}\right],
$$

em que

$$
\boldsymbol{H}_{5 i}=\sum_{l=1}^{N_{Q G H}} \sum_{m=1}^{N_{Q G H}} e^{g(\cdot)}\left[\boldsymbol{W}_{2} \boldsymbol{W}_{2}^{\top}+\operatorname{diag}\left(\boldsymbol{K}_{i}+\boldsymbol{L}_{i}+\boldsymbol{M}_{i}\right)\right] \frac{w_{l} w_{m}}{\pi}
$$


com

$$
\begin{aligned}
\boldsymbol{K}_{i} & =\left(\delta_{i 1} \frac{\partial D_{i 1}}{\partial \eta_{2 i 1}}, \ldots, \delta_{i n_{i}} \frac{\partial D_{i n_{i}}}{\partial \eta_{2 i n_{i}}}\right)^{\top}, \\
\boldsymbol{L}_{i} & =\left(\log \left(y_{i 1}\right) \frac{\partial \sigma_{i 1}^{\star}}{\partial \eta_{2 i 1}}, \ldots, \log \left(y_{i n_{i}}\right) \frac{\partial \sigma_{i n_{i}}^{\star}}{\partial \eta_{2 i n_{i}}}\right)^{\top}, \\
\boldsymbol{M}_{i} & =\left(\frac{\partial E_{i 1}}{\partial \eta_{2 i 1}}, \ldots, \frac{\partial E_{i n_{i}}}{\partial \eta_{2 i n_{i}}}\right)^{\top} .
\end{aligned}
$$

Assim, a matriz obtida ao derivar $l(\boldsymbol{\theta})$ duas vezes em relação ao vetor $\boldsymbol{\beta}_{1}$ é a seguinte:

$$
\frac{\partial^{2} l(\boldsymbol{\theta})}{\partial \boldsymbol{\beta}_{2}^{2}}=\sum_{i=1}^{N} \boldsymbol{X}_{2 i}^{\top}\left[\frac{-\boldsymbol{C}_{2 i} \boldsymbol{C}_{2 i}^{\top}}{P_{i}^{2}}+\frac{\boldsymbol{H}_{5 i}}{P_{i}}\right] \boldsymbol{X}_{2 i} .
$$

Derivando $l(\boldsymbol{\theta})$ duas vezes, primeiro em relação à $r$-ésima componente de $\boldsymbol{\beta}_{2}$ e depois em relação a $\tau_{1}$, temos que

$$
\frac{\partial^{2} l(\boldsymbol{\theta})}{\partial \tau_{1} \partial \boldsymbol{\beta}_{2 r}}=\sum_{i=1}^{N}\left[\frac{-\boldsymbol{X}_{2 i, r}^{\top} \boldsymbol{C}_{2 i} C_{3 i}}{P_{i}^{2}}+\frac{\boldsymbol{X}_{2 i, r}^{\top} \boldsymbol{H}_{6 i}}{P_{i}}\right]
$$

em que

$$
\begin{aligned}
\boldsymbol{E}_{6 i} & =\sum_{l=1}^{N_{Q G H}} \sum_{m=1}^{N_{Q G H}} e^{g(\cdot)}\left[W_{3} \boldsymbol{W}_{2}+\boldsymbol{R}_{i}+\boldsymbol{T}_{i}+\boldsymbol{U}_{i}\right] \frac{w_{l} w_{m} \sqrt{2} p_{l}}{\pi} \\
\boldsymbol{R}_{i} & =\left(\delta_{i 1} \frac{\partial D_{i 1}}{\partial \eta_{1 i 1}}, \ldots, \delta_{i n_{i}} \frac{\partial D_{i n_{i}}}{\partial \eta_{1 i n_{i}}}\right)^{\top}, \\
\boldsymbol{T}_{i} & =\left(\log \left(y_{i 1}\right) \frac{\partial \sigma_{i 1}^{\star}}{\partial \eta_{1 i 1}}, \ldots, \log \left(y_{i n_{i}}\right) \frac{\partial \sigma_{i n_{i}}^{\star}}{\partial \eta_{1 i n_{i}}}\right)^{\top} \\
\boldsymbol{U}_{i} & =\left(\frac{\partial E_{i 1}}{\partial \eta_{1 i 1}}, \ldots, \frac{\partial E_{i n_{i}}}{\partial \eta_{1 i n_{i}}}\right)^{\top}
\end{aligned}
$$

Assim, a matriz obtida ao derivar $l(\boldsymbol{\theta})$ duas vezes, primeiro em relação a $\boldsymbol{\beta}_{2}$ e depois em relação a $\tau_{1}$, é a seguinte:

$$
\frac{\partial^{2} l(\boldsymbol{\theta})}{\partial \tau_{1} \partial \boldsymbol{\beta}_{2}}=\sum_{i=1}^{N} \boldsymbol{X}_{2 i}^{\top}\left[\frac{-\boldsymbol{C}_{2 i} C_{3 i}}{P_{i}^{2}}+\frac{\boldsymbol{H}_{6 i}}{P_{i}}\right] .
$$

Derivando $l(\boldsymbol{\theta})$ duas vezes, primeiro em relação à $r$-ésima componente de $\boldsymbol{\beta}_{2}$ e depois em 
relação a $\tau_{2}$, temos que

$$
\frac{\partial^{2} l(\boldsymbol{\theta})}{\partial \tau_{2} \partial \boldsymbol{\beta}_{2 r}}=\sum_{i=1}^{N}\left[\frac{-\boldsymbol{X}_{2 i, r}^{\top} \boldsymbol{C}_{2 i} C_{4 i}}{P_{i}^{2}}+\frac{\boldsymbol{X}_{2 i, \cdot r}^{\top} \boldsymbol{H}_{7 i}}{P_{i}}\right]
$$

em que

$$
\boldsymbol{H}_{7 i}=\sum_{l=1}^{N_{Q G H}} \sum_{m=1}^{N_{Q G H}} e^{g(\cdot)}\left[W_{4} \boldsymbol{W}_{2}+\boldsymbol{K}_{i}+\boldsymbol{L}_{i}+\boldsymbol{M}_{i}\right] \frac{w_{l} w_{m} \sqrt{2} p_{m}}{\pi} .
$$

Assim, a matriz obtida ao derivar $l(\boldsymbol{\theta})$ duas vezes, primeiro em relação a $\boldsymbol{\beta}_{2}$ e depois em relação a $\tau_{2}$, é a seguinte:

$$
\frac{\partial^{2} l(\boldsymbol{\theta})}{\partial \tau_{2} \partial \boldsymbol{\beta}_{2}}=\sum_{i=1}^{N} \boldsymbol{X}_{2 i}^{\top}\left[\frac{-\boldsymbol{C}_{2 i} C_{4 i}}{P_{i}^{2}}+\frac{\boldsymbol{H}_{7 i}}{P_{i}}\right]
$$

Derivando $l(\boldsymbol{\theta})$ duas vezes em relação a $\tau_{1}$ temos que

$$
\frac{\partial^{2} l(\boldsymbol{\theta})}{\partial \tau_{1}^{2}}=\sum_{i=1}^{N}\left[\frac{-C_{3 i}^{2}}{P_{i}^{2}}+\frac{H_{8 i}}{P_{i}}\right]
$$

em que

$$
H_{8 i}=\sum_{l=1}^{N_{Q G H}} \sum_{m=1}^{N_{Q G H}} e^{g(\cdot)}\left[W_{3}^{2}+\boldsymbol{F}_{i}-\boldsymbol{G}_{i}\right] \frac{w_{l} w_{m} 2 p_{l}^{2}}{\pi} .
$$

Derivando $l(\boldsymbol{\theta})$ duas vezes, primeiro em relação a $\tau_{2}$ e depois em relação a $\tau_{1}$, temos que

$$
\frac{\partial^{2} l(\boldsymbol{\theta})}{\partial \tau_{1} \partial \tau_{2}}=\sum_{i=1}^{N}\left[\frac{-C_{3 i} C_{4 i}}{P_{i}^{2}}+\frac{H_{9 i}}{P_{i}}\right]
$$

em que

$$
H_{9 i}=\sum_{l=1}^{N_{Q G H}} \sum_{m=1}^{N_{Q G H}} e^{g(\cdot)}\left[W_{3} W_{4}+\left(\boldsymbol{R}_{i}+\boldsymbol{T}_{i}+\boldsymbol{U}_{i}\right)^{\top} \mathbf{1}_{n_{i}}\right] \frac{w_{l} w_{m} 2 p_{l} p_{m}}{\pi} .
$$

Derivando $l(\boldsymbol{\theta})$ duas vezes em relação a $\tau_{2}$ temos que

$$
\frac{\partial^{2} l(\boldsymbol{\theta})}{\partial \tau_{2}^{2}}=\sum_{i=1}^{N}\left[\frac{-C_{4 i}^{2}}{P_{i}^{2}}+\frac{H_{10 i}}{P_{i}}\right]
$$


106 APÊNDICE C

em que

$$
H_{10 i}=\sum_{l=1}^{N_{Q G H}} \sum_{m=1}^{N_{Q G H}} e^{g(\cdot)}\left[W_{4}^{2}+\left(\boldsymbol{K}_{i}+\boldsymbol{L}_{i}-\boldsymbol{M}_{i}\right)^{\top} \mathbf{1}_{n_{i}}\right] \frac{w_{l} w_{m} 2 p_{m}^{2}}{\pi} .
$$




\section{Referências Bibliográficas}

Agresti et al.(2004) A. Agresti, B. Caffo e P. Ohman-Strickland. Examples in which misspecification of a random effects distribution reduces efficiency, and possible remedies. Computational Statistics \& Data Analysis, 47:639-653. Citado na pág. 64, 67

Aitkin e Longford(1986) M. Aitkin e N. Longford. Statistical modeling issues in school effectiveness studies. Journal of the Royal Statistical Society, Series A, 149:1-43. Citado na pág. 3

Aitkin et al.(1981) M. Aitkin, D. Anderson e J. Hinde. Statistical modelling of data on teaching styles (with discussion). Journal of the Royal Statistical Society, Series A, 144: 144-161. Citado na pág. 3

Alonso et al.(2008) A. Alonso, S. Litière e G. Molenberghs. A family of tests to detect misspecifications in the random-effects structure of generalized linear mixed models. Computational Statistical \& Data Analysis, 52:4474-4486. Citado na pág. 67

Alonso et al.(2010) A. Alonso, S. Litière e G. Molenberghs. Testing for misspecification in generalized linear mixed models". Biostatistics, 11(4):771-786. Citado na pág. 67

Avalos et al.(1993) B. R. Avalos, J. L. Kapoor, N. Tutschka, P. J. Klein e E. A. Copelan. Preparation of bone marrow transplantation in Hodgkin's and non-Hodgkin's lymphoma using Bu/Cy. Bone Marrow Transplantation, 13:133-138. Citado na pág. 28

Bates et al.(2012) D. Bates, M. Maechler e B. Bolker. lme4: Linear mixed-effects models using S4 classes, 2012. URL http://CRAN.R-project.org/package=lme4. R package version 0.999999-0. Citado na pág. 82, 84

Blocker(2011) Alexander W Blocker. fastGHQuad: Fast Rcpp implementation of GaussHermite quadrature, 2011. URL http://CRAN.R-project.org/package=fastGHQuad. R package version $0.1-1$. Citado na pág. 74

Breslow e Clayton(1993) N. Breslow e D. G. Clayton. Approximate inference in generalized linear mixed models. Journal of the American Statistical Association, 88:9-25. Citado na pág. 63 
Broström e Holmberg(2011a) G. Broström e H. Holmberg. Generalized linear models with clustered data: Fixed and random effects models. Computational Statistics and Data Analysis, 55:3123-3134. Citado na pág. 60

Broström e Holmberg(2011b) G. Broström e H. Holmberg. glmmML: Generalized linear models with clustering, 2011b. URL http://CRAN.R-project.org/package=glmmML. R package version $0.82-1$. Citado na pág. 74

Burstein(1980) L. Burstein. The analysis of multilevel data in educational research and evaluation. Review of Research in Education, 8:158-233. Citado na pág. 3

Colosimo e Giolo(2006) E. A. Colosimo e S. R. Giolo. Análise de sobrevivência aplicada. Blucher, Sao Paulo. Citado na pág. 93

Crainiceanu e Ruppert(2004) C. M. Crainiceanu e D. Ruppert. Likelihood ratio tests in linear mixed models with one variance component. Journal of the Royal Statistical Society Series $B, 6(1): 165-185$. Citado na pág. 18

D'Agostinho e Stephens(1986) R. D'Agostinho e M Stephens. Goodness-of-fit techniques. Marcel Dekker, New York. Citado na pág. 27

de Leeuw e Meijer(2008) J. de Leeuw e E Meijer. Introduction to multilevel analysis. Em J. de Leeuw e E Meijer, editors, Handbook of Multilevel Analysis. primeira edição. Citado na pág. 3,5

Demidenko(2004) E. Demidenko. Mixed Model, Theory and Applications. John Wiley \& Sons, Inc, New Jersey. Citado na pág. 8, 13, 74

Dempster et al.(1981) A. Dempster, D. Rubin e R. Tsutakawa. Estimation in covariance components models. Journal of the American Statistical Association, 76:341-353. Citado na pág. 3

Dunn e Smyth(1996) P. Dunn e G. Smyth. Randomized Quantile Residuals. Journal of Computational and Graphical Statistics, 5(3):236-244. Citado na pág. 17

Fabio et al.(2012) L. Fabio, G. Paula e M. de Castro. A Poisson mixed model with nonnormal random effect distribution. Computational Statistical and Data Analysis, 56: 1499-1510. Citado na pág. 41

Gelman e Hill(2007) A. Gelman e J. Hill. Data Analysis Using Regression and Multilevel/Hierarchical Models. Cambridge University, New York, segunda edição. Citado na pág. 5

Giampaoli e Singer(2009) V. Giampaoli e J. M. Singer. Likelihood ratio tests for variance components in linear mixed models. Journal of Statistical Planning and Inference, 139: 1435-1448. Citado na pág. 18 
Goldstein(2011) H. Goldstein. Multilevel Statistical Model. John Wiley \& Sons, Ltd, quarta edição. Citado na pág. 1

Hanagal(2011) D. D. Hanagal. Modeling survival data using frailty models. CRC A Chapman \& Hall, Boca Raton. Citado na pág. 28

Hartley e Rao(1967) H. Hartley e J. Rao. Maximum likelihood estimation for the mixed analysis of variance model. Biometrika, 54:93-108. Citado na pág. 3

Hartzel et al.(2001) H. Hartzel, A. Agresti e B. Caffo. Multinomial logit random effects models. Statistical Modelling, 1:81-102. Citado na pág. 78

Heagerty e Kurland(2001) P. J. Heagerty e B. F. Kurland. Misspecified Maximum Likelihood Estimates and Generalised Linear Mixed Models. Biometrika, 88(4):973-985. Citado na pág. 64,69

Hedeker(2008) D. Hedeker. Multilevel models for ordinal and nominal variables. Em J. de Leeuw e E Meijer, editors, Handbook of Multilevel Analysis. primeira edição. Citado na pág. 3

Hernández et al.(2013) F. Hernández, O. Usuga e V. Giampaoli. Improving the Adaptive Gaussian Quadrature. Janeiro 2013. Citado na pág. 79

Huang(2009) X. Huang. Diagnosis of random-effect model misspecification in generalized linear mixed models for binary response. Biometrics, 65:361-368. Citado na pág. 63

Johnson et al.(1994) N. L. Johnson, S. Kotz e N. Balakrishnan. Continuous univariate distributions. New York: Wiley. Citado na pág. 64

Laird e Ware(1982) N. Laird e H. Ware. Random-effects models for longitudinal data. Biometrics, 38:963-974. Citado na pág. 3, 7

Lee et al.(2006) Y. Lee, J. Nelder e Yudi Pawitan. Generalized Linear Models with Random Effects, Unified Analysis via H-likelihood. Chapman \& Hall/CRC, Boca Raton. Citado na pág. 41

Lindley e Smith(1972) D. Lindley e A. Smith. Bayes estimates for the linear model. Journal of the Royal Statistical Society, Series B, 34:1-41. Citado na pág. 3

Litière et al.(2007) S. Litière, A. Alonso e G. Molenberghs. Type I and type II error under random-effects misspecification in generalized linear mixed models. Biometrics, 63: 1038-1044. Citado na pág. 64, 65, 67

Litière et al.(2008) S. Litière, A. Alonso e G. Molenberghs. The impact of a misspecified random-effects distribution on the estimation and the performance of inferential procedures in generalized linear mixed models. Statistics in Medicine, 27:3125-3144. Citado na pág. 64,67 
Litière et al.(2011) S. Litière, A. Alonso e G. Molenberghs. Rejoinder to "A Note on Type II Error Under Random Effects Misspecification in Generalized Linear Mixed Models". Biometrics, 67(2):656-660. Citado na pág. 65, 67

Liu e Yu(2008) L. Liu e Z. Yu. A likelihood reformulation method in non-normal random effects models. Statistics in Medicine, 27(3):3105-3124. Citado na pág. 44, 60

Liu e Pierce(1994) Q. Liu e D. A. Pierce. A Note on Gauss-Hermite Quadrature. Biometrika, 81(3):624-629. Citado na pág. 75

Longford(1987) N. Longford. A fast scoring algorithm for maximum likelihood estimation in unbalanced models with nested random effects. Biometrika, 74(4):817-827. Citado na pág. 3

Manghi(2011) Roberto Manghi. Modelos elípticos multiníveis. Dissertação de Mestrado, Instituto de Matematica e Estatística, Universidade de São Paulo, Brasil. Citado na pág. 3

McCulloch et al.(2008) C. McCulloch, S. Searle e J. Neuhaus. Generalized, Linear, and Mixed Models. Wiley, New York, segunda edição. Citado na pág. 16

McCulloch e Neuhaus(2011a) C. E. McCulloch e J. M. Neuhaus. Misspecifying the shape of a random effects distribution: Why getting it wrong may not matter. Statistical Science, 26:388-342. Citado na pág. 63, 64, 69

McCulloch e Neuhaus(2011b) C. E. McCulloch e J. M. Neuhaus. Prediction of random effects in linear and generalized linear models under model misspecification. Biometrics, 67:270-279. Citado na pág. 47, 65, 67, 69

Molenberghs e Verbeke(2005) G. Molenberghs e G. Verbeke. Models for Discrete Longitudinal Data. Springer, New York, primeira edição. Citado na pág. 7, 15

Morris e Christiansen(1995) C. Morris e C. Christiansen. Fitting Weibull duration models with random effects. Lifetime Data Analysis, 1(4):347-359. Citado na pág. 41

Neuhaus et al.(1992) J. M. Neuhaus, W. W. Hauck e J. D. Kalbfleisch. The Effects of Mixture Distribution Misspecification when Fitting Mixed-Effects Logistic Models. Biometrika, 79(4):755-762. Citado na pág. 64, 69

Neuhaus et al.(2011) J. M. Neuhaus, C. E. McCulloch e R. Boylan. A note on Type II error under random effects misspecifications in generalized linear mixed models. Biometrics, 67 (2):654-660. Citado na pág. 65, 92

Neuhaus et al.(2012) J. M. Neuhaus, C. E. McCulloch e R Boylan. Estimation of covariate effects in generalized linear mixed models with a misspecified distribution of random intercepts and slopes. Statistics in Medicine. , available online. Citado na pág. 65 
Ortega et al.(2008) E. Ortega, G. Paula e H. Bolfarine. Deviance residuals in generalised log-gamma regression models with censored observations. Journal of Statistical Computation and Simulation, 78(8):747-764. Citado na pág. 16

Ortega et al.(2011) E. Ortega, G. Cordeiro e E. Hashimoto. A Log-Linear Regression Model for the Beta-Weibull Distribution. Communications in Statistics - Simulation and Computation, 40(8):1206-1235. Citado na pág. 16, 17

Pinheiro e Bates(1995) J. C. Pinheiro e D. M. Bates. Approximations to the LogLikelihood Function in the Nonlinear Mixed-Effects Model. Journal of Computational and Graphical Statistics, 4(1):12-35. Citado na pág. 75

Pinheiro e Chao(2006) J. C. Pinheiro e E. C. Chao. Efficient Laplacian and Adaptive Gaussian Quadrature Algorithms for Multilevel Generalized Linear Mixed Models. Journal of Computational and Graphical Statistics, 15(1):58-81. Citado na pág. 13, 66

R Development Core Team(2012) R Development Core Team. R: A Language and Environment for Statistical Computing. R Foundation for Statistical Computing, Vienna, Austria, 2012. URL http://www.R-project.org. ISBN 3-900051-07-0. Citado na pág. 1

Rasbash e Browne(2008) J. Rasbash e W. Browne. Non-hierarchical multilevel models. Em J. de Leeuw e E Meijer, editors, Handbook of Multilevel Analysis. Citado na pág. 3

Raudenbush(2008) S. Raudenbush. Many small groups. Em J. de Leeuw e E Meijer, editors, Handbook of Multilevel Analysis. Citado na pág. 3

Raudenbush e Bryk(2002) S. Raudenbush e A. Bryk. Hierarchical Linear Models: Applications and Data Analysis Methods. Sage Publications, California, segunda edição. Citado na pág. $1,4,5,6$

Ribeiro et al.(2012) P. J. Ribeiro, W. H. Bonat, E. T. Krainski e W. M. Zeviani. Métodos Computacionais em Inferência Estatística. Laboratório de Estatística e GeoinformaÃฐão (LEG), 2012. URL http://www.leg.ufpr.br/〜 paulojus/mcie/masterSINAPE-2012-08-13. pdf. Citado na pág. 78

Rigby e Stasinopoulos(2005) R. Rigby e D. Stasinopoulos. Generalized additive models for location, scale and shape. Applied Statistics, 54(3):507-554. Citado na pág. 1, 11, 17, 82, 84,86

Rodriguez(2008) G. Rodriguez. Multilevel generalized linear models. Em J. de Leeuw e E Meijer, editors, Handbook of Multilevel Analysis. Citado na pág. 3

Rosenberg(1967) B. Rosenberg. Linear regression with randomly dispersed parameters. Biometrika, 60:61-75. Citado na pág. 3 
Russo et al.(2012) C. M. Russo, R. Aoki e G. A Paula. Assessment of variance components in nonlinear mixed-effects elliptical models. TEST, 21(3):519-545. Citado na pág. 92

Sahu et al.(1997) S. Sahu, D. Dey, H. Aslanidou e D. Sinha. A Weibull Regression Model with Gamma Frailties for Multivariate Survival Data. Lifetime Data Analysis, 3(2):123137. Citado na pág. 41

Savalli et al.(2006) C. Savalli, G. A. Paula e Cysneiros F. J. Assessment of variance components in elliptical linear mixed models. Statistical Modelling, 6:59-76. Citado na pág. 92

Searle et al.(2006) Shayle Searle, George Casella e Charles McCulloch. Variance Components. Wiley, New York, segunda edição. Citado na pág. 16

Shapiro(1988) A. Shapiro. Towards a unified theory of inequality constrained testing in multivariate analysis. International Statistical Review, 56:49-62. Citado na pág. 17

Skrondal e Rabe-Hesketh(2009) A. Skrondal e S. Rabe-Hesketh. Prediction in Multilevel Generalized Linear Models. Journal of the Royal Statistical Society. Series A (Statistics in Society), 172(3):659-687. Citado na pág. 3

Smith(1973) A. Smith. A general Bayesian lineal model. Journal of the Royal Statistical Society, Series B, 35:61-75. Citado na pág. 3

Smyth et al.(2011) G. Smyth, Y. Hu, P. Dunn e B. Phipson. statmod: Statistical Modeling, 2011. URL http://CRAN.R-project.org/package=statmod. R package version 1.4.14. Citado na pág. 74

Spiegelhalter et al.(2003) D. Spiegelhalter, A. Thomas, N. Best e Lunn D. WinBUGS version 1.4 User Manual, 2003. URL http://www.mrc-bsu.cam.ac.uk/bugs/. Citado na pág. 85

Spiegelhalter et al.(2012) D. Spiegelhalter, A. Thomas, N. Best e Lunn D. OpenBUGS User Manual, 2012. URL http://www.cran.r-project.org/web/packages/dclone/dclone. pdf. R package version 18.1-1. Citado na pág. 85

Stasinopoulos e Rigby(2012) Mikis Stasinopoulos e Bob Rigby. Package gamlss.dist. Available at http://www.cran.r-project.org/web/packages/gamlss.dist/gamlss.dist.pdf, 2012. Citado na pág. 9, 66

Stram e Lee(1994) D. O. Stram e J. W. Lee. Variance Components Testing in the Longitudinal Mixed Effects Model. Biometrics, 50(4):1171-1177. Citado na pág. 17

Stram e Lee(1995) D. O. Stram e J. W. Lee. Correction to "Variance Components Testing in the Longitudinal Mixed Effects Model". Biometrics, 51(4):1196. Citado na pág. 17 
Therneau(2012) T. Therneau. A Package for Survival Analysis in S, 2012. R package version 2.36-14. Citado na pág. 83

Therneau et al.(1990) T. Therneau, P. Grambsch e T. Fleming. Martingale-Based Residuals for Survival Models. Biometrika, 77(1):147-160. Citado na pág. 16

Verbeke e Lesaffre(1997) G. Verbeke e E. Lesaffre. The effect of misspecifiying the randoeffects distribution in linear mixed models for longitudinal data. Computational Statistics E Data Analysis, 23:541-556. Citado na pág. 63, 67, 68

Verbeke e Molenberghs(2000) G. Verbeke e G. Molenberghs. Linear Mixed Models for Longitudinal Data. Springer, New York. Citado na pág. 8

Villegas(2010) C. Villegas. Modelos Log-Birnbaum-Saunders Mistos. Tese de Doutorado, Instituto de Matemática e Estatística, Universidade de São Paulo, Brasil. Citado na pág. 18

Villegas et al.(2011) C. Villegas, G. Paula e V. Leiva. Birnbaum-Sanders Mixed Models for Censored Reliability Data Analysis. IEEE Transactions on Reliability, 60(4):748-758. Citado na pág. 16,18

Vu e Zhou(1997) H. T. V. Vu e S. Zhou. Generalization of Likelihood Ratio Test under Nonstandard Conditions. The Annals of Statistics, 25(2):897-916. Citado na pág. 18

Weibull(1939) W. Weibull. A statistical theory of the strength of materials. Ingeniors Vetenskaps Akademien Handlingar, 151:1-45. Citado na pág. 93

Wissel(2009) J. Wissel. A new biased estimator for multivariate regression models with highly collinear variables. Tese de Doutorado, Universität Würzburg, Am Hubland, 97074 Würzburg. Citado na pág. 20

Zhang et al.(2008) P. Zhang, P. Song, A. Qu e T. Greene. Efficient Estimation for PatientSpecific Rates of Disease Progression Using Nonnormal Linear Mixed Models. Biometrics, 64:29-38. Citado na pág. 41 